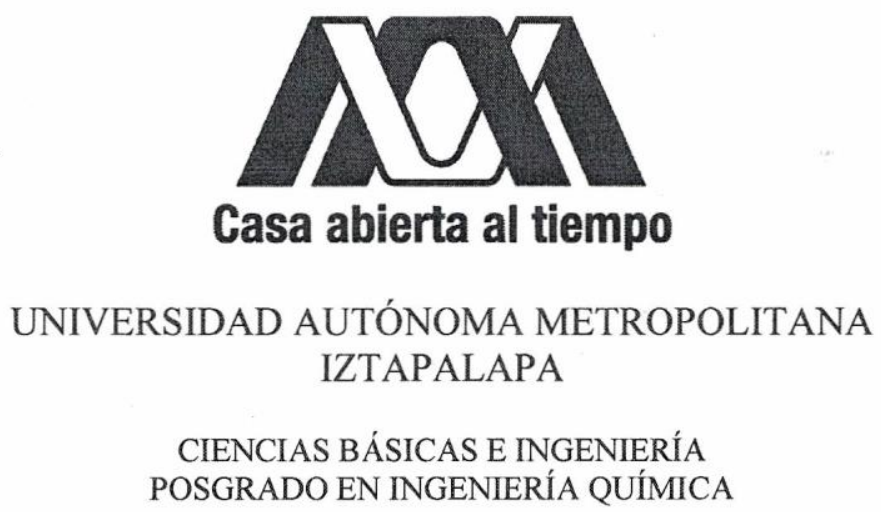

\title{
Síntesis de redes de intercambio de calor mediante la generación y optimización de topologías redundantes
}

Tesis para obtener el grado de:

\section{Maestro en Ciencias en Ingeniería Química}

Presentada por:

\section{Luis Eduardo Pedroza Robles Arenas}

Bajo la asesoría de:

\section{Dr. Juan Manuel Zamora Mata}

Jurado Calificador:

Presidente:

Dr, Juan Manuel Zamora Mata

Secretario:

Vocal:

Dr. Miguel Ángel Gutiérrez Limón

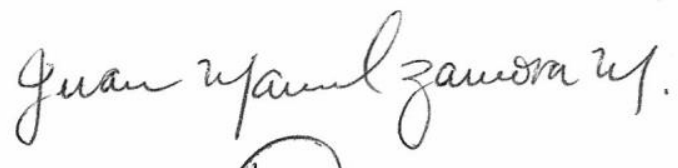

Dr. Rogelio Hernández Suárez

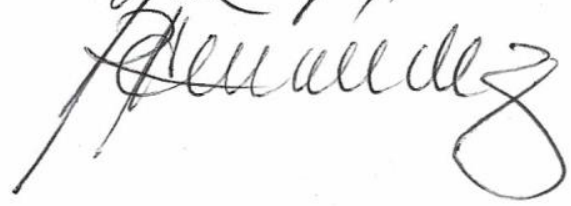

Ciudad de México, Febrero de 2018 



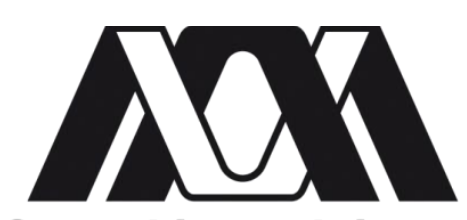

Casa abierta al tiempo

UNIVERSIDAD AUTÓNOMA METROPOLITANA
IZTAPALAPA

CIENCIAS BÁSICAS E INGENIERÍA

POSGRADO EN INGENIERÍA QUÍMICA

\section{Síntesis de redes de intercambio de calor mediante la generación y optimización de topologías redundantes}

Tesis para obtener el grado de:

Maestro en Ciencias en Ingeniería Química

Presentada por:

Luis Eduardo Pedroza Robles Arenas

Bajo la asesoría de:

Dr. Juan Manuel Zamora Mata

Jurado Calificador:

Presidente: $\quad$ Dr. Juan Manuel Zamora Mata

Secretario: $\quad$ Dr. Miguel Ángel Gutiérrez Limón

Vocal: $\quad$ Dr. Rogelio Hernández Suárez 



\section{Agradecimientos}

A mis padres por su incondicional amor y confianza, las palabras no son suficientes para expresar mi gratitud. Su ejemplo y constante aliento han sido la inspiración para perseguir mis ambiciones. Sin duda no hubiera logrado esto sin ustedes.

A mi hermana Melissa y a su esposo Gilberto por compartir tanto conmigo, me han enseñado que siempre se puede ser una mejor persona. Por la convivencia, la compañía, los ánimos y el apoyo para seguir mis estudios, y sobre todo gracias su amistad.

A Mara, por su constante y sincero amor que fortaleció mi moral al estar a mi lado en todas las situaciones. Las palabras optimistas y el valor que me has transmitido para hacer cosas nuevas me han hecho creer que cualquier cosa es posible. Gracias por todas las experiencias que nos han unido y nos han hecho crecer juntos.

A mis amigos que han sido una importante influencia en mi vida. Aunque es imposible mencionar a todos, quiero agradecer especialmente a Rodrigo, a Carlos y a Tania por acompañarme a lo largo de mi estadía en la universidad. Gracias por las pláticas, los consejos, las motivaciones y las memorables anécdotas que siempre tendré en mi memoria, pero lo más importante, gracias por la gran compañía que siempre me han brindado.

A mi asesor el Dr. Juan Manuel Zamora Mata por el interés, las valiosas sugerencias y continua orientación que me han motivado a superarme en mi formación académica. Así como por la paciencia y tiempo dedicado al desarrollo y revisión de este trabajo.

Al Consejo nacional de ciencia y tecnología (CONACYT) por la beca de estudios de posgrado otorgada para el desarrollo de este trabajo de investigación.

A la Universidad Autónoma Metropolitana-Iztapalapa por brindarme la oportunidad para desarrollarme en una etapa más de mi formación profesional.

Al laboratorio de Síntesis, Optimización y Simulación de Procesos del Departamento de Ingeniería de Procesos e Hidráulica, por las facilidades otorgadas. 


\section{Resumen}

En este trabajo se desarrolla una nueva metodología secuencial para la síntesis de redes de intercambio de calor. El enfoque de síntesis propuesto tiene como objetivo determinar diseños de red económicos buscando superar las limitaciones de las metodologías de síntesis secuenciales actuales. La metodología presentada consiste principalmente de dos etapas. En la primera etapa, se genera un diseño de red base con un número relativamente grande de intercambiadores de calor y sub-corrientes de proceso. Se presentan tres esquemas diferentes para generar diseños de red redundantes con diferentes niveles de redundancia en términos de la cantidad de equipos de intercambio de calor. En la segunda etapa, se utiliza un algoritmo de optimización con multiarranque estocástico para aumentar las probabilidades de determinar el mejor diseño de red disponible, contenido en el diseño base. La aplicación de la metodología se ilustra con la solución de siete casos de estudio de la literatura. Los resultados muestran que los diseños desarrollados con la metodología propuesta presentan costos competitivos con respecto a los diseños de red reportados en la literatura.

En el Capítulo 1 se presenta una breve introducción, así como el estado del arte en relación con la síntesis de redes de intercambio de calor. En el Capítulo 2 se describe el problema de uso mínimo de servicios auxiliares formulado como un modelo de transporte; este método de programación lineal representa un elemento clave en la metodología desarrollada debido a su capacidad de proporcionar múltiples conjuntos de apareamientos entre corrientes de proceso, que pueden ser interpretados como equipos de intercambio de calor. El algoritmo de multi-arranque estocástico considerado como el segundo elemento clave en la metodología de síntesis de redes de intercambio de calor propuesta, se describe en el Capítulo 3; este algoritmo iterativo, elimina subconjuntos de equipos de intercambio de calor redundantes de un diseño base y evita la terminación en un único óptimo local de baja calidad. En el Capítulo 4 se definen reglas y algoritmos para generar diseños base redundantes con diferentes niveles de redundancia. En el Capítulo 5 se ilustra la aplicación de la metodología de síntesis propuesta resolviendo siete problemas de referencia. Los resultados incluyen tres nuevos diseños de red que mejoran las soluciones previamente reportadas en la literatura; el resto de los diseños desarrollados presentan un costo total anual con menos del $1 \%$ por arriba de las soluciones conocidas para cada caso de 
estudio respectivamente. Finalmente, en el Capítulo 6 se resumen las contribuciones de este trabajo de investigación, y se presentan posibles áreas de desarrollo para un trabajo a futuro. 


\section{Abstract}

A novel sequential methodology for the synthesis of heat exchanger networks is developed in this work. The proposed synthesis approach aims at determining economical network designs by overcoming current limitations of the sequential synthesis methodologies. The methodology presented consists of two main stages. In the first stage a base network design with a relatively substantial number of heat exchange units and process sub-streams is generated. Three different schemes for generating redundant base network designs are presented, with distinct levels of redundancy in terms of the number of heat exchangers. In the second stage a stochastic multistart optimization algorithm is utilized to increase the probabilities to determine the best possible network design embedded in the base network design. The application of the proposed synthesis methodology is illustrated with the solution of seven case of study from the literature. The results show that the designs developed with the proposed synthesis methodology are competitive in terms of total annual costs with respect to network designs from the literature.

Chapter 1 presents a brief introduction and the state of the art of the synthesis of heat exchanger networks. Chapter 2 describes the problem of minimum utility usage formulated as a transportation problem; this linear programming based method serves as one of the key points of the presented methodology due to its capability for providing multiple sets of matches between process streams, which can be interpreted as heat exchanger units. The stochastic multi-start optimization algorithm is described in Chapter 3; this algorithm, which iteratively eliminates different sub-sets of redundant heat exchange units from the base network designs and avoids termination into a single low quality local optimum, is considered as the second fundamental element of the proposed HEN synthesis methodology. Chapter 4 defines rules and algorithms to generate the redundant base network designs with distinct levels of redundancy. Chapter 5 illustrates the application of the proposed synthesis methodology to seven benchmark problems. The results include three new best network designs that overcome previous solutions from the literature; the rest of the developed designs present total annual costs within $1 \%$ of the bestknown solutions of the literature for the respective benchmark problems. Finally, Chapter 6 summarizes the contributions of this research work, as well as possible areas for future work. 


\section{Índice}

Resumen
Abstract
iii

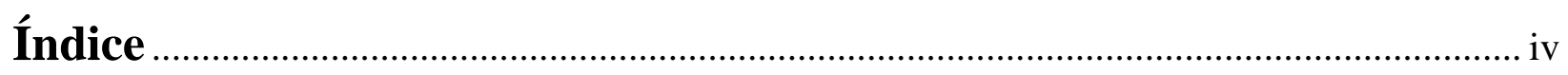

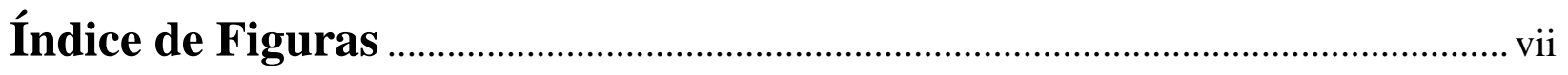

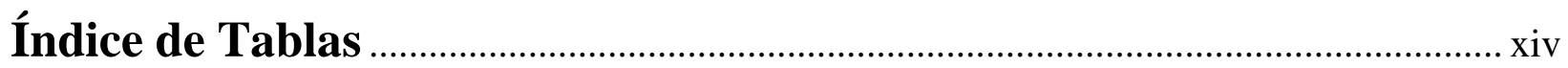

\section{Capítulo 1. Introducción}

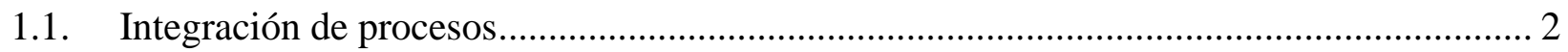

1.2. Síntesis de redes de intercambio de calor. ………........................................................ 3

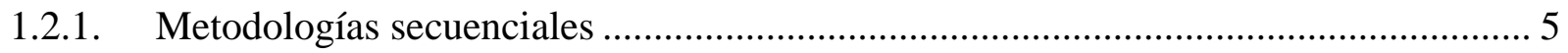

1.2.2. Metodologías simultáneas .................................................................................. 10

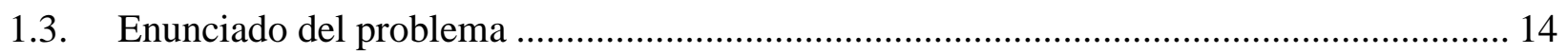

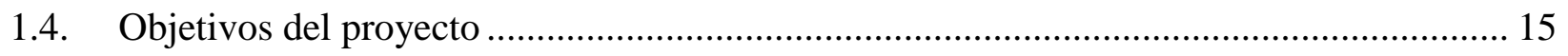

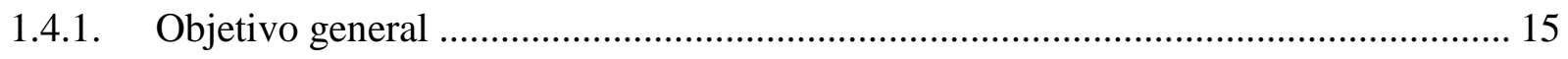

1.4.2. Objetivos particulares ..................................................................................... 16

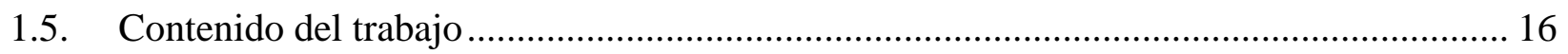

\section{Capítulo 2. Modelo de Transporte}

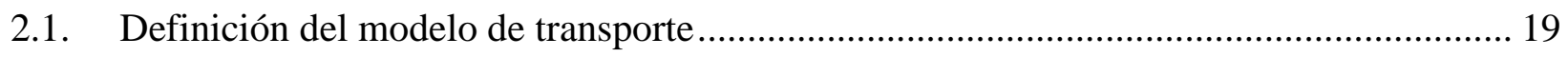

2.2. Problema del requerimiento mínimo de servicios auxiliares............................................. 22

2.3. Problema del número mínimo de apareamientos ................................................................ 27

2.4. Alternativa al problema de número mínimo de apareamientos ......................................... 30 


\section{Capítulo 3. Optimización de redes de intercambio de calor a partir de un diseño base}

3.1. Metodologías para la optimización de redes de intercambio de calor ............................. 34

3.2. Algoritmo de optimización multi-arranque estocástico en dos fases............................. 38

3.3. Estructuras redundantes como diseño base para la optimización .................................. 44

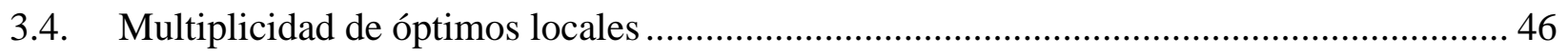

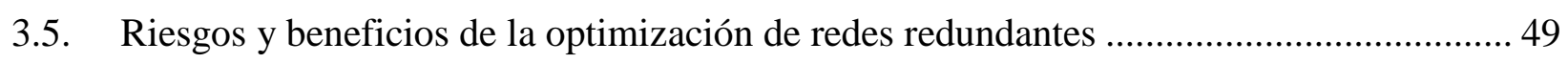

\section{Capítulo 4. Metodología secuencial para la síntesis de redes de intercambio de calor}

4.1. Redes redundantes de intercambio de calor ............................................................. 55

4.2. Distribución de cargas térmicas para la generación de topologías redundantes .................56

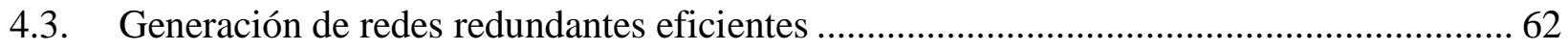

4.4. Variación del HRAT para generación de diferentes redes redundantes .......................... 83

4.5. Metodología secuencial para la síntesis de redes de intercambio de calor ...................... 84

\section{Capítulo 5. Resultados}

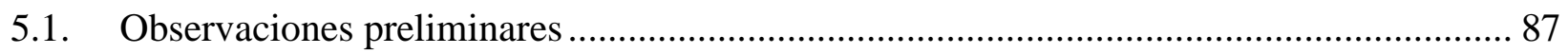

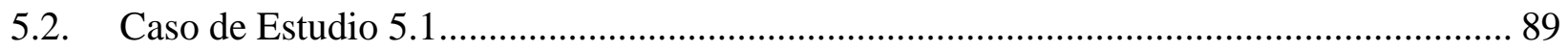

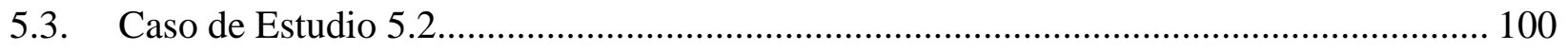

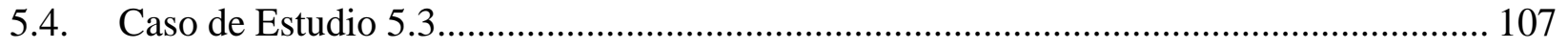

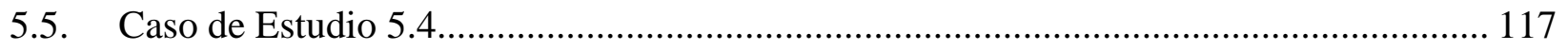

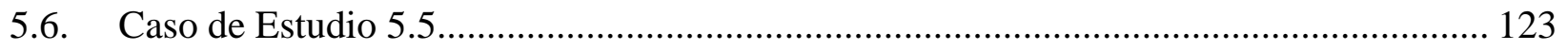

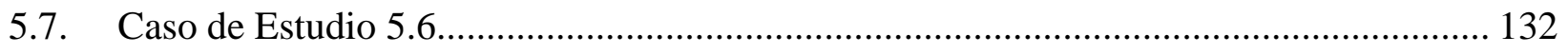

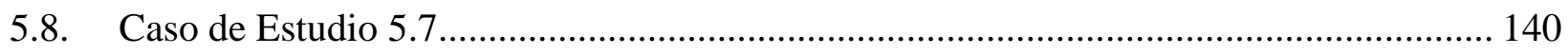


5.9. Resumen de resultados.

\section{Capítulo 6. Conclusiones y trabajo a futuro}

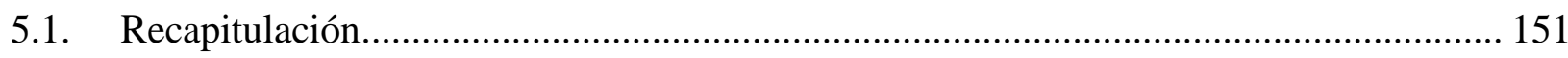

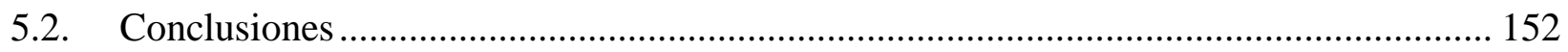

5.3. Posibles líneas de investigación en un trabajo a futuro ............................................ 158

\section{Apéndice A}

A.1. Revisión del Caso de Estudio 5.1 ........................................................................ 160

A.2. Revisión del Caso de Estudio 5.2 ......................................................................... 165

A.3. Revisión del Caso de Estudio 5.3 ........................................................................ 170

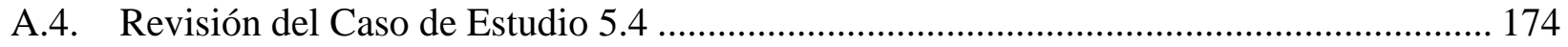

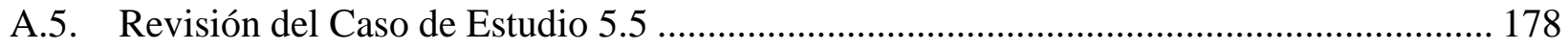

A.6. Revisión del Caso de Estudio 5.6 ................................................................................... 184

A.7. Revisión del Caso de Estudio 5.7 ......................................................................... 190

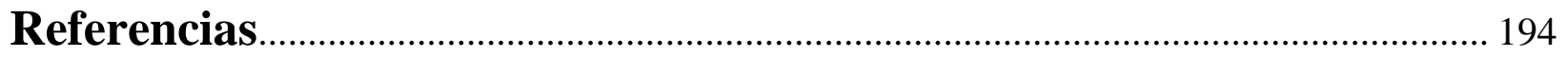




\section{Índice de Figuras}

Figura 1.1. Modelo de cebolla para el diseño de un proceso (Smith, 2005)............................. 2

Figura 2.1. Representación de red del modelo de transporte. $\quad 20$

Figura 2.2. Representación gráfica del modelo de transporte para el problema de uso mínimo de

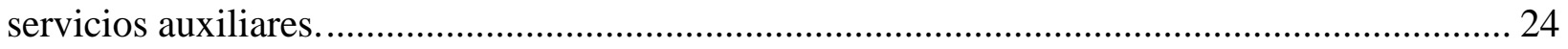

Figura 2.3. Representación de los nodos de servicios auxiliares para un HRAT igual a $10{ }^{\circ} \mathrm{C}$... 25

Figura 2.4. Ejemplos de notación sobre el diagrama de temperaturas para la formulación del

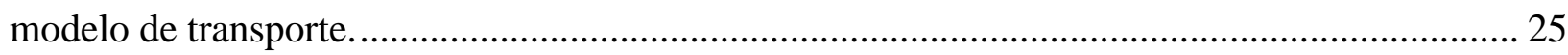

Figura 3.1. Representación de una red de intercambio de calor con estructura fija. $\quad 39$

Figura 3.2. Algoritmo de optimización de multi-arranque estocástico en dos fases (Núñez-Serna

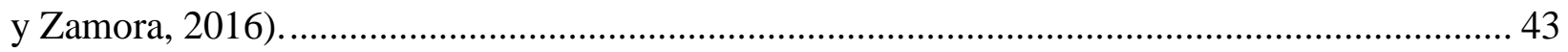

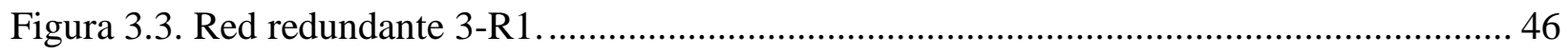

Figura 3.4. Histograma de soluciones óptimas locales obtenidas fijando 150 inicializaciones para

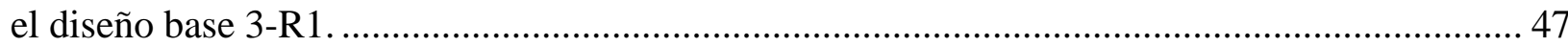

Figura 3.5. Diferentes soluciones óptimas locales contenidas en la estructura 3-R1, para el ejemplo ilustrativo 3.1(temperaturas $\left({ }^{\circ} \mathrm{C}\right)$ en fuente normal, cargas térmicas $(\mathrm{kW})$ en cursivas y

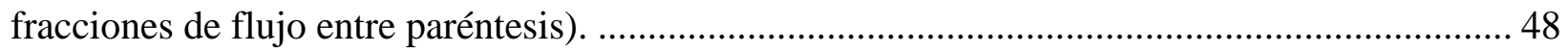

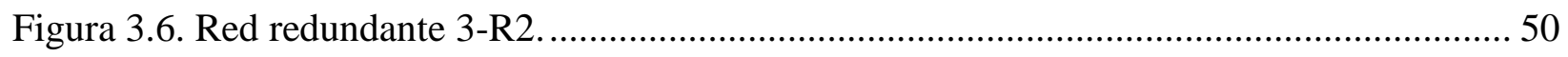

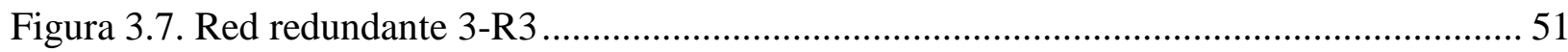

Figura 3.8. Histograma de soluciones óptimas locales obtenidas fijando 250 inicializaciones para

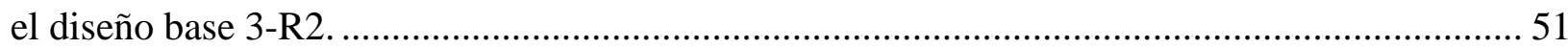

Figura 3. 9. Histograma de soluciones óptimas locales obtenidas fijando 250 inicializaciones para

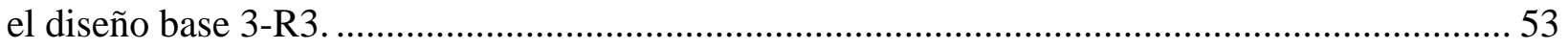


Figura 3.10. Mejor solución óptima local con $\mathrm{CTA}_{\text {Paterson }}$ de $\$ 418,398.36$ contenida en la estructura 3-R3, para el ejemplo ilustrativo 3.1 (temperaturas $\left({ }^{\circ} \mathrm{C}\right.$ ) en fuente normal, cargas térmicas $(\mathrm{kW})$ en cursivas y fracciones de flujo entre paréntesis)........................................ 54

Figura 4.1. Rutas para el intercambio de calor entre corrientes de proceso y servicios auxiliares. 58

Figura 4.2. Representación de la distribución de cargas térmicas. 59

Figura 4.3. Diseño de red redundante sin división de corrientes, basado en los intercambios de calor de la solución del modelo de transporte. 59

Figura 4.4. Topología redundante sin división de corrientes, basada en intervalos de temperatura. 61

Figura 4.5. Dos filas comparadas que pertenecen a la misma corriente, si los equipos se encuentran sobre la misma etapa deben ser colocados en sub-corrientes diferentes. 65

Figura 4.6. Equipo en fila inferior puede ser colocado en serie si se encuentra en una etapa posterior al último equipo de la fila bajo análisis. 66

Figura 4.7. La primera fila no permite equipos sobre las etapas 2 y 3 al contener un intercambiador de calor respectivamente. 66

Figura 4.8. Equipos 3 y 7 correspondientes a $\mathrm{H} 2$, no fueron admitidos en etapas de la primera fila, por lo que son colocados en serie sobre una sub-corriente diferente. 67

Figura 4.9. El equipo 9 es colocado en serie dejando una entrada vacía. 70

Figura 4.10. El equipo 10 no cumple con los criterios para ser colocado en serie en la

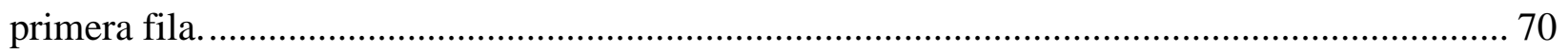

Figura 4.11. Diagrama de malla la topología redundante 1 para el ejemplo ilustrativo 4.1 ........ 72 Figura 4.12. Integración de dos apareamientos para definir una distribución de cargas térmicas reducida. 74

Figura 4.13. Apareamiento que permanece igual en la distribución de cargas térmicas reducida. 75 
Figura 4.14. Integración de tres apareamientos para definir una distribución de cargas térmicas reducida. 75

Figura 4.15. Equipos colocados en serie utilizando un esquema reducido de apareamientos...... 77 Figura 4.16. Diagrama de malla de la red redundante integración de apareamientos para el ejemplo ilustrativo 4.1 . 79

Figura 4.17. Integración de tres apareamientos para definir una distribución de cargas térmicas sin reapareamientos. 81

Figura 4.18. Diagrama de malla de la red redundante sin reapareamientos para el ejemplo ilustrativo 4.1 . 82

Figura 4.19. Algoritmo para la generación y optimización de topologías redundantes para la síntesis de redes de intercambio de calor. 85

Figura 5.1. "Super-targeting" y selección del intervalo de HRAT para la generación de topologías redundantes en el Caso de Estudio 5.1. 90

Figura 5.2. Primera ocurrencia de cada solución óptima local, de la optimización de una topología redundante con el tercer nivel de redundancia y HRAT de $4{ }^{\circ} \mathrm{C}$ realizando 1000 inicializaciones para el Caso de Estudio 5.1..... 92 Figura 5.3. Óptimos locales de menor CTA Paterson para cada HRAT en el Caso de Estudio 5.1. . 94 Figura 5.4. Soluciones óptimas locales de menor CTA Paterson para cada HRAT en el Caso de Estudio 5.1, prohibiendo los servicios auxiliares a las corrientes de proceso $\mathrm{H} 1$ y C1.............. 96

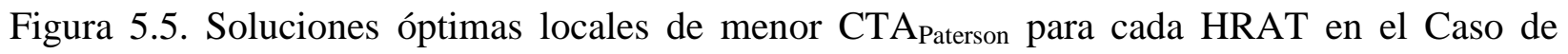
Estudio 5.1 prohibiendo los servicios auxiliares a las corrientes de proceso H1 y C2............... 98 Figura 5.6. Topología redundante generada con un HRAT de $7{ }^{\circ} \mathrm{C}$ y el primer nivel de redundancia para el Caso de Estudio 5.1. 98

Figura 5.7. Mejor solución óptima local para el Caso de Estudio 5.1 con CTA de \$411,624.48 (temperaturas $\left({ }^{\circ} \mathrm{C}\right)$ en fuente normal, cargas térmicas $(\mathrm{kW})$ en cursivas y fracciones de flujo entre paréntesis). 99 
Figura 5.8. "Super-Targeting" y selección del intervalo de HRAT para la generación de topologías redundantes en el Caso de Estudio 5.2 ........................................................... 102

Figura 5.9. Óptimos locales de menor CTA ${ }_{\text {Paterson }}$ para cada HRAT en el Caso de Estudio 5.2. 103

Figura 5.10. Soluciones óptimas locales de menor CTA Paterson para cada HRAT en el Caso de Estudio 5.2, prohibiendo los servicios auxiliares a las corrientes de proceso $\mathrm{H} 2$ y $\mathrm{C} 1$. 105

Figura 5.11. Topología redundante generada con un HRAT de $21{ }^{\circ} \mathrm{C}$ y el primer nivel de redundancia para el Caso de Estudio 5.2. 106

Figura 5.12. Mejor solución óptima local para el Caso de Estudio 5.2 con CTA de \$80,847 (temperaturas $\left({ }^{\circ} \mathrm{C}\right)$ en fuente normal, cargas térmicas $(\mathrm{kW})$ en cursivas y fracciones de flujo entre paréntesis). 106

Figura 5.13. "Super-Targeting" y selección del intervalo de HRAT para la generación de topologías redundantes para el Caso de Estudio 5.3... 109

Figura 5.14. Óptimos locales de menor CTA $_{\text {Paterson para cada HRAT en el }}$ Caso de Estudio 5.3. 111

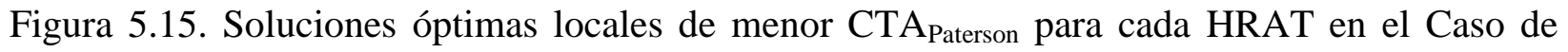
Estudio 5.3, prohibiendo los servicios auxiliares a las corrientes de proceso H4, C1 y C2...... 112 Figura 5.16. Búsqueda intensiva de soluciones óptimas locales para cada HRAT en el Caso de Estudio 5.3, prohibiendo los servicios auxiliares a las corrientes de proceso H1, C2 y C3...... 115 Figura 5.17. Topología redundante generada con un HRAT de $12.96{ }^{\circ} \mathrm{C}$ y el tercer nivel de redundancia para el Caso de Estudio 5.3. 115

Figura 5.18. Mejor solución óptima local para el Caso de Estudio 5.3 con CTA de \$105,481.12 (temperaturas $\left({ }^{\circ} \mathrm{C}\right)$ en fuente normal, cargas térmicas $(\mathrm{kW})$ en cursivas y fracciones de flujo entre paréntesis). 116

Figura 5.19. "Super-Targeting" y selección del intervalo de HRAT para la generación de topologías redundantes para el Caso de Estudio 5.4. 119

Figura 5.20. Óptimos locales de menor CTA Paterson para cada HRAT en el Caso de Estudio 5.4. 120 
Figura 5.21. Topología redundante generada con un HRAT de $5.9{ }^{\circ} \mathrm{F}$ y el segundo nivel de redundancia para el Caso de Estudio 5.4. 121

Figura 5.22. Mejor solución óptima local para el Caso de Estudio 5.4 con CTA de \$29,156.65 (temperaturas $\left({ }^{\circ} \mathrm{F}\right)$ en fuente normal, cargas térmicas (kBtu h-1) en cursivas y fracciones de flujo entre paréntesis).

Figura 5.23. "Super-Targeting" y selección del intervalo de HRAT para la generación de topologías redundantes para el Caso de Estudio 5.5...... 125

Figura 5.24. Óptimos locales de menor CTAPaterson para cada HRAT en el Caso de Estudio 5.5. 126

Figura 5.25. Búsqueda intensiva de soluciones óptimas locales para cada HRAT en el Caso de Estudio 5.5, prohibiendo servicios auxiliares a las corrientes de proceso H2, C2, C3 y C4..... 128

Figura 5.26. Topología redundante generada con un HRAT de $12{ }^{\circ} \mathrm{C}$ y una búsqueda intensiva usando el primer nivel de redundancia para el Caso de Estudio 5.5.

Figura 5.27. Mejor solución óptima local para el Caso de Estudio 5.5 con CTA de \$2,919,687.66 (temperaturas $\left({ }^{\circ} \mathrm{C}\right)$ en fuente normal, cargas térmicas $(\mathrm{kW})$ en cursivas y fracciones de flujo entre paréntesis). 130

Figura 5.28. "Super-Targeting" y selección del intervalo de HRAT para la generación de topologías redundantes para el Caso de Estudio 5.6. 134

Figura 5.29. Óptimos locales de menor CTAPaterson para cada HRAT en el Caso de Estudio 5.6. 135

Figura 5.30. Búsqueda intensiva de soluciones óptimas locales para cada HRAT en el Caso de Estudio 5.6, prohibiendo los servicios auxiliares a las corrientes de proceso H1, H2 y H3...... 137 Figura 5.31. Topología redundante generada con un HRAT de $12 \mathrm{~K}$ y una búsqueda intensiva usando el primer nivel de redundancia para el Caso de Estudio 5.6. 137

Figura 5.32. Mejor solución óptima local para el Caso de Estudio 5.6 con CTA de \$43,317.97 (temperaturas $(\mathrm{K})$ en fuente normal, cargas térmicas $(\mathrm{kW})$ en cursivas y fracciones de flujo entre paréntesis). 138 
Figura 5.33. "Super-Targeting" y selección del intervalo de HRAT para la generación de topologías redundantes para el Caso de Estudio 5.7. 142

Figura 5.34. Óptimos locales de menor CTAPaterson para cada HRAT en el Caso de Estudio 5.7. 144

Figura 5.35. Búsqueda intensiva de soluciones óptimas locales para cada HRAT en el Caso de Estudio 5.7, prohibiendo los servicios auxiliares a las corrientes de proceso C6, H1, H2, H3, H5, H6 y $\mathrm{H} 7$. 145

Figura 5.36. Topología redundante generada con un HRAT de $12.71{ }^{\circ} \mathrm{C}$ y una búsqueda intensiva usando el primer nivel de redundancia para el Caso de Estudio 5.7. 146

Figura 5.37. Mejor solución óptima local para el Caso de Estudio 5.7 con CTA de \$1,519,049.24 (temperaturas $\left({ }^{\circ} \mathrm{C}\right)$ en fuente normal, cargas térmicas $(\mathrm{kW})$ en cursivas y fracciones de flujo entre paréntesis). 147

Figura A.1. Diseño de red determinado por Zamora y Grossmann (1997) para el Caso de Estudio 5.1 con CTA $_{\text {DMLT }}$ reportado de $\$ 419,979$ y CTA DMLT $_{\text {revisado de } \$ 419,978.82 .} 160$

Figura A.2. Diseño de red determinado por Björk y Westerlund (2002) para el Caso de Estudio

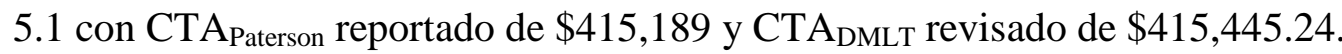
160

Figura A.3. Diseño de red determinado en este trabajo para el Caso de Estudio 5.1 con CTA DMLT de $\$ 411,624.48$. 163

Figura A.4. Diseño de red determinado por Petterson (2008) para el Caso de Estudio 5.2 con CTA $_{\text {DMLT }}$ reportado de $\$ 80,962$ y CTA $_{\text {DMLT }}$ revisado de $\$ 80,959.55$. 165

Figura A.5. Diseño de red determinado por Gupta y Ghosh (2010) para el Caso de Estudio 5.2 con $\mathrm{CTA}_{\text {Paterson }}$ reportado de $\$ 80,379$ y $\mathrm{CTA}_{\mathrm{DMLT}}$ revisado de $\$ 80,378.98$. 165

Figura A.6. Diseño de red determinado en este trabajo para el Caso de Estudio 5.2 con CTA DMLT de $\$ 80,847.71$. 168

Figura A.7. Diseño de red determinado por Huang y Karimi (2013) con CTA Paterson reportado de

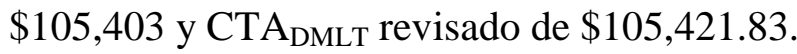
170

Figura A.8. Diseño de red determinado en este trabajo para el Caso de Estudio 5.3 con CTA DMLT de $\$ 105,481.12$. 172 
Figura A.9. Diseño de red determinado por Luo y col. (2004) para el Caso de Estudio 5.4 con $\mathrm{CTA}_{\mathrm{DMLT}}$ reportado de $\$ 29,438$ y $\mathrm{CTA}_{\mathrm{DMLT}}$ revisado de $\$ 29,310.88$. 174

Figura A.10. Diseño de red determinado en este trabajo para el Caso de Estudio 5.4 con CTA $_{\text {DMLT }}$ de $\$ 29,156.65$. 176

Figura A.11. Diseño de red determinado por Petterson (2005) para el Caso de Estudio 5.5 con $\mathrm{CTA}_{\mathrm{DMLT}}$ reportado de $\$ 2,904,953$ y $\mathrm{CTA}_{\mathrm{DMLT}}$ revisado de $\$ 2,904,953.09$. 178

Figura A.12. Diseño de red determinado por Pavão y col. (2017b) para el Caso de Estudio 5.5

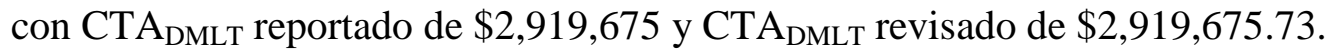
180

Figura A.13. Diseño de red determinado en este trabajo para el Caso de Estudio 5.5 con CTA $A_{\text {DMLT }}$ de $\$ 2,919,687.66$. 182

Figura A.14. Diseño de red determinado por Wu y col. (2015) para el Caso de Estudio 5.6 con

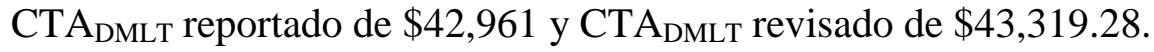
184

Figura A.15. Diseño de red determinado por Lin y Miller (2004) para el Caso de Estudio 5.6 con $\mathrm{CTA}_{\mathrm{DMLT}}$ reportado de $\$ 42,329$ y $\mathrm{CTA}_{\mathrm{DMLT}}$ revisado de $\$ 43,329.24$. 186

Figura A.16. Diseño de red determinado en este trabajo para el Caso de Estudio 5.6 con CTA $_{\text {DMLT }}$ de $\$ 43,317.79$. 188

Figura A.17. Diseño de red determinado por Escobar y Trierweiler (2013) para el Caso de Estudio 5.7 con CTA $_{\text {Chen }}$ reportado de $\$ 1,506,667.04$ y CTA DMLT $_{\text {revisado de } \$ 1,505,262.18 .190}$

Figura A.18. Diseño de red determinado en este trabajo para el Caso de Estudio 5.7 con CTA $_{\text {DMLT }}$ de $\$ 1,519,049.24$. 192 


\section{Índice de Tablas}

Tabla 2. 1. Datos de corrientes del Ejemplo ilustrativo 2.1.................................................. 23

Tabla 3.1. Datos de corrientes y costos del ejemplo ilustrativo 3.1.45

Tabla 3.2. Resumen de soluciones óptimas locales que contiene el diseño base 3-R1, fijando 150

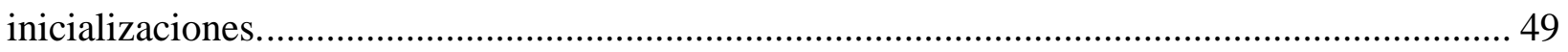

Tabla 3.3. Soluciones óptimas locales para el diseño base 3-R2 fijando 2000 inicializaciones... 52

Tabla 4.1. Datos de corrientes y costos del ejemplo ilustrativo 4.1.57

Tabla 4.2. Intercambios de calor obtenidos de la solución del modelo de transporte.

Tabla 4.3. Distribución de cargas térmicas ordenada con respecto a los intervalos de temperatura calientes.

Tabla 4.4. Representación inicial por etapas de equipos en las corrientes calientes. 64

Tabla 4.5. Distribución final de equipos en sub-corrientes de proceso calientes. 68

Tabla 4.6. Representación inicial por etapas de equipos en las corrientes frías. 69

Tabla 4.7. Distribución final de equipos en sub-corrientes de proceso frías. 71

Tabla 4.8. Distribución de cargas térmicas reducida 76

Tabla 4.9. Distribución de cargas térmicas reducida, ordenada con respecto a los intervalos iniciales de temperatura calientes. 76

Tabla 4.10. Representación inicial por etapas de equipos en las corrientes calientes con

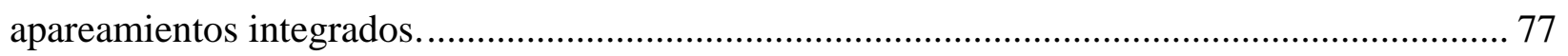

Tabla 4.11. Distribución final de equipos en sub-corrientes de proceso calientes, con

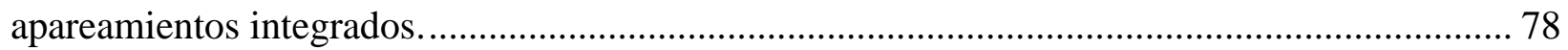

Tabla 4.12. Representación inicial por etapas de equipos en las corrientes frías con

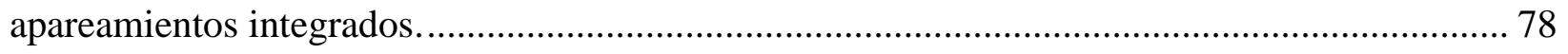

Tabla 4.13. Distribución final de equipos en sub-corrientes de proceso calientes, con apareamientos integrados 
Tabla 4.14. Distribución de cargas térmicas sin reapareamientos. 81

Tabla 4.15. Distribución de cargas térmicas sin reapareamientos, ordenada 82

Tabla 5.1. Datos de corrientes y costos para el Caso de Estudio 5.1.

89

Tabla 5.2. Valores estimados por el método de "Super-Targeting” para el Caso de Estudio 5.1. 91

Tabla 5.3. Resultados del Caso de Estudio 5.1. 93

Tabla 5.4. Comparación de las mejores soluciones obtenidas con soluciones de alto costo para el Caso de Estudio 5.1. 95

Tabla 5.5. Resultados del Caso de Estudio 5.1 prohibiendo servicios auxiliares a las corrientes de proceso $\mathrm{H} 1$ y $\mathrm{C} 2$....... 97

Tabla 5.6. Comparación del Caso de Estudio 5.1 con soluciones reportadas en la literatura..... 100

Tabla 5.7. Datos de corrientes y costos para el Caso de Estudio 5.2. 101

Tabla 5.8. Valores estimados por el método de "Super-Targeting" para el

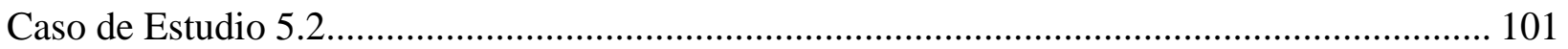

Tabla 5.9. Resultados del Caso de Estudio 5.2 .............................................................. 103

Tabla 5.10. Resultados del Caso de Estudio 5.2 prohibiendo servicios auxiliares a las corrientes

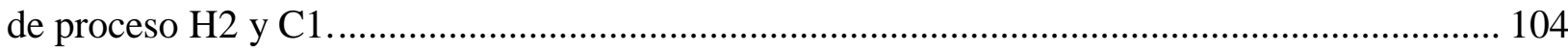

Tabla 5.11. Comparación del Caso de Estudio 5.2 con soluciones reportadas en la literatura... 107

Tabla 5.12. Datos de corrientes y costos para el Caso de Estudio 5.3................................... 108

Tabla 5.13. Valores estimados por el método de "Super-Targeting" para el

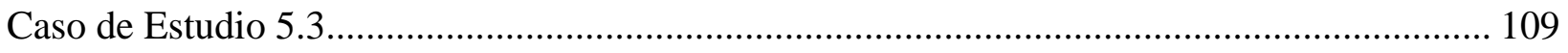

Tabla 5.14. Resultados del Caso de Estudio 5.3 .............................................................. 110

Tabla 5.15. Resultados del Caso de Estudio 5.3 prohibiendo servicios auxiliares a las corrientes

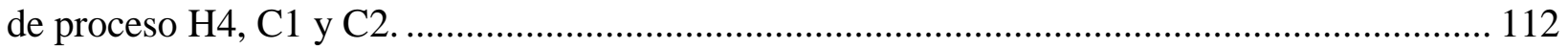

Tabla 5.16. Resultados del Caso de Estudio 5.3 prohibiendo servicios auxiliares a las corrientes de proceso e intensificando la búsqueda.......................................................................... 113

Tabla 5.17. Comparación del Caso de Estudio 5.3 con soluciones reportadas en la literatura... 117 
Tabla 5.18. Datos de corrientes y costos para el Caso de Estudio 5.4. 117

Tabla 5.19. Valores estimados por el método de "Super-Targeting" para el

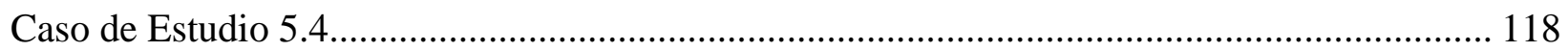

Tabla 5.20. Resultados del Caso de Estudio 5.4............................................................. 120

Tabla 5.21. Comparación del Caso de Estudio 5.4 con soluciones reportadas en la literatura... 123

Tabla 5.22. Datos de corrientes y costos para el Caso de Estudio 5.5.................................... 124

Tabla 5.23. Valores estimados por el método de "Super-Targeting" para el

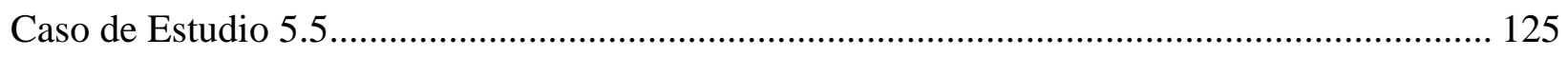

Tabla 5.24. Resultados del Caso de Estudio 5.5 ................................................................ 126

Tabla 5.25. Resultados del Caso de Estudio 5.5 prohibiendo servicios auxiliares a las corrientes de proceso e intensificando la búsqueda....................................................................... 127

Tabla 5.26. Comparación del Caso de Estudio 5.5 con soluciones reportadas en la literatura... 131

Tabla 5.27. Datos de corrientes y costos para el Caso de Estudio 5.6.................................. 132

Tabla 5.28. Valores estimados por el método de "Super-Targeting" para el

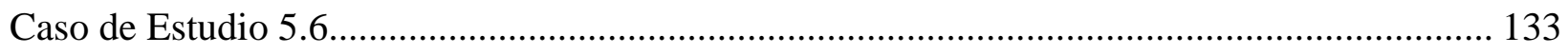

Tabla 5.29. Resultados del Caso de Estudio 5.6................................................................... 135

Tabla 5.30. Resultados del Caso de Estudio 5.6 prohibiendo servicios auxiliares a las corrientes de proceso e intensificando la búsqueda....................................................................... 136

Tabla 5.31. Comparación del Caso de Estudio 5.6 con soluciones reportadas en la literatura... 139

Tabla 5.32. Datos de corrientes y costos para el Caso de Estudio 5.7 .................................. 141

Tabla 5.33. Valores estimados por el método de "Super-Targeting" para el

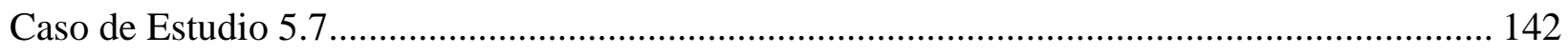

Tabla 5.34. Resultados del Caso de Estudio 5.7 .................................................................... 143

Tabla 5.35. Resultados del Caso de Estudio 5.7 prohibiendo servicios auxiliares a las corrientes de proceso e intensificando la búsqueda.......................................................................... 145 Tabla 5.36. Comparación del Caso de Estudio 5.7 con soluciones reportadas en la literatura... 147 
Tabla 5.37. Resumen de resultados para los siete casos de estudio abordados en este trabajo. . 150

Tabla A.1 Revisión de costos del diseño de red de la Figura A.1 desarrollado por Zamora y Grossmann (1997) para el Caso de Estudio 5.1. 161

Tabla A.2 Revisión de costos del diseño de red de la Figura A.2 desarrollado por Björk y Westerlund (2002) para el Caso de Estudio 5.1 ..................................................................... 162

Tabla A.3 Revisión de costos del diseño de red de la Figura A.3 desarrollado en este trabajo para el Caso de Estudio 5.1 164

Tabla A.4 Revisión de costos del diseño de red de la Figura A.4 desarrollado por Petterson (2008) para el Caso de Estudio 5.2. 166

Tabla A.5 Revisión de costos del diseño de red de la Figura A.5 desarrollado por Gupta y Ghosh (2010) para el Caso de Estudio 5.2. 167

Tabla A.6 Revisión de costos del diseño de red de la Figura A.6 desarrollado en este trabajo para el Caso de Estudio 5.2. 169

Tabla A.7 Revisión de costos del diseño de red de la Figura A.7 desarrollado por Luo y col. (2004) para el Caso de Estudio 5.3. 171

Tabla A.8 Revisión de costos del diseño de red de la Figura A.8 desarrollado en este trabajo para el Caso de Estudio 5.3. 173

Tabla A.9 Revisión de costos del diseño de red de la Figura A.9 desarrollado por Luo y col. (2004) para el Caso de Estudio 5.4. 175

Tabla A.10 Revisión de costos del diseño de red de la Figura A.10 desarrollado en este trabajo para el Caso de Estudio 5.4.

Tabla A.11 Revisión de costos del diseño de red de la Figura A.11 desarrollado por Petterson (2005) para el Caso de Estudio 5.5. 179

Tabla A.12 Revisión de costos del diseño de red de la Figura A.12 desarrollado por Pavão y col. (2017b) para el Caso de Estudio 5.5. 181

Tabla A.13 Revisión de costos del diseño de red de la Figura A.12 desarrollado en este trabajo para el Caso de Estudio 5.5. 183 
Tabla A.14 Revisión de costos del diseño de red de la Figura A.14 desarrollado por Wu y col. (2015) para el Caso de Estudio 5.6. 185

Tabla A.15 Revisión de costos del diseño de red de la Figura A.14 desarrollado por Lin y Miller (2004) para el Caso de Estudio 5.6. 187

Tabla A.16 Revisión de costos del diseño de red de la Figura A.15 desarrollado en este trabajo para el Caso de Estudio 5.6. 189

Tabla A.17 Revisión de costos del diseño de red de la Figura A.17 desarrollado por Escobar y Trierweiler (2013) para el Caso de Estudio 5.7. 191 Tabla A.18 Revisión de costos del diseño de red de la Figura A.18 desarrollado en este trabajo

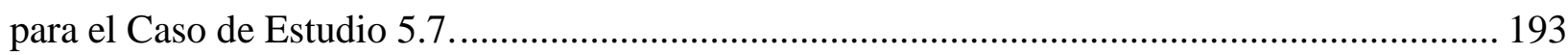






\section{Capítulo 1. Introducción}

El daño al medio ambiente que la explotación de combustibles fósiles ha ocasionado, es un tema que ha cobrado especial interés en las últimas décadas. Una innovada conciencia ha surgido a raíz del perceptible decaimiento que ha sufrido el planeta a consecuencia del cambio climático. Por estas razones, gobiernos de diferentes países han impulsado la disminución del consumo de energías provenientes de combustibles fósiles, imponiendo límites en las emisiones contaminantes e invirtiendo en tecnologías basadas en la generación de energías limpias.

A pesar de estas medidas, de acuerdo con el "BP statistical review of world energy (2016)" las emisiones de dióxido de carbono se han incrementado en un 17.4\% tan sólo del 2005 al 2015, siendo el petróleo el principal combustible, contando con el 33\% del consumo energético global. Aunado a esto, las reservas de petróleo probadas al 2015, alcanzan los 1697.6 billones de barriles, suficientes para cubrir las necesidades actuales por 50.7 años. Por otro lado, las energías renovables representan únicamente el $2.8 \%$ del consumo global, colocando esta alternativa muy alejada a cumplir con las crecientes demandas energéticas a precios asequibles. Estos son claros indicadores de que la dependencia de energías convencionales se mantendrá al menos a corto plazo.

De acuerdo con la Administración de Información de Energía de los Estados Unidos (2016), el sector industrial se sitúa como el principal consumidor de energía, con un uso de poco más de la mitad de la demanda global. Puesto que está previsto que algunos países en vías de desarrollo contribuyan de forma progresiva al crecimiento industrial, y debido a que cada vez más productos se vuelven de uso esencial, es posible proyectar que la demanda energética del sector industrial se vea incrementada con el paso del tiempo. Por estas razones el uso eficiente de la energía impone un reto de atención inmediata cuyos beneficios se pueden traducir en términos tanto ambientales como en incentivos económicos. 


\subsection{Integración de procesos}

Durante las últimas décadas se han destinado esfuerzos en desarrollar técnicas que permitan la implementación de procesos disminuyendo el consumo de recursos y las emisiones contaminantes al medio ambiente. La integración de procesos representa un enfoque que provee una visión global de un proceso químico como un sistema integrado, relacionando las interconexiones entre unidades y corrientes (El-Halwagi, 1997). En términos más generales, la integración de procesos desarrolla procedimientos que permiten el diseño y optimización desde sistemas individuales hasta plantas de proceso, buscando un equilibrio entre la reducción de costos y la disminución de la materia y energía requerida.

Un proceso químico integrado se puede visualizar con el diagrama de "cebolla" mostrado en la Figura 1.1 (Smith, 2005), donde las tomas de decisiones deben ser tomadas jerárquicamente, partiendo del diseño del reactor como el elemento principal del sitio total, y diseñando sucesivamente el resto de las "capas" del proceso. Las interconexiones entre cada parte del sistema están dadas por las corrientes de proceso, las cuales requieren energía para llevar a cabo reacciones u otras operaciones unitarias con el fin de transformar la materia prima en productos químicos.

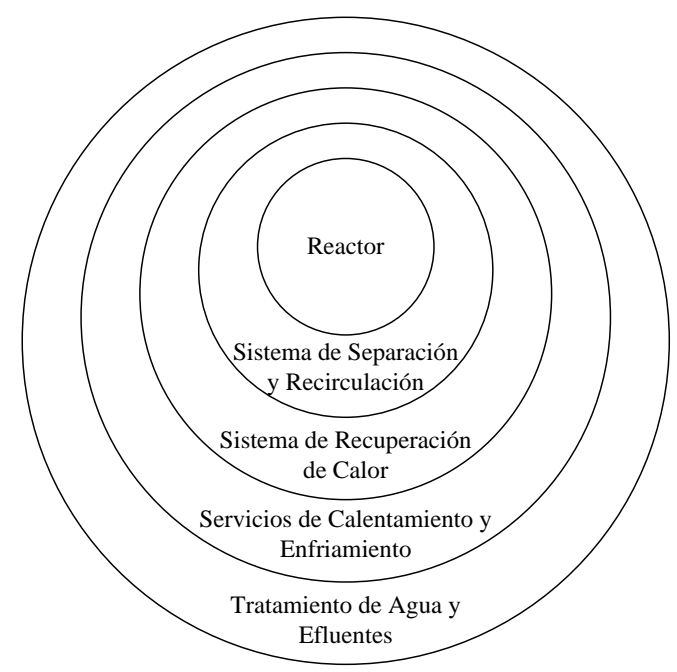

Figura 1.1. Modelo de cebolla para el diseño de un proceso (Smith, 2005).

Las metodologías de integración de procesos se remontan a los años 70s, en respuesta a la existente crisis de petróleo, que causaba la escasez y encarecimiento de combustibles (Klemeš, 
2013). Ésto impulsó la implementación de técnicas que permitieran usar los recursos disponibles de forma más eficiente. Hohmann (1971) realizó un trabajo pionero en el contexto de la integración térmica, donde se definen por primera vez conceptos para la recuperación de energía entre las corrientes de una planta de proceso, logrando reducir el requerimiento de energía externa. Estos elementos pueden ser considerados como el inicio de la integración de procesos, así como los fundamentos de la metodología de punto de pliegue, la cual permanece vigente como el procedimiento estándar en proyectos industriales para la identificación en oportunidades de ahorro energético. La definición formal del punto de pliegue se atribuye principalmente a Linnhoff y Flower (1978a, 1978b, 1979) y a Umeda y col. (1978) quienes simultáneamente desarrollaron trabajos para la determinación de metas de recuperación de energía. Estas ideas son retomadas posteriormente para evolucionar a lo que se conoce como tecnología de punto de pliegue (Linnhoff y Hindmarsh, 1983).

Analogías del punto de pliegue han sido trasladadas a otras áreas de estudio para el uso eficiente de recursos; como sistemas de intercambio de masa (El-Halwagi y Manousiouthakis, 1989), minimización de aguas residuales y sistemas de tratamiento distribuido de efluentes (Wang y Smith, 1994), gestión de hidrógeno en refinerías de petróleo (Towler y col., 1996), plantas de tratamiento biológico de aguas residuales (Zhelev y Ntlhakana, 1999) y satisfacción de requisitos de energía que cumplan con los límites de emisión de $\mathrm{CO}_{2}$ en el sector energético (Tan y Foo, 2007).

\subsection{Síntesis de redes de intercambio de calor.}

En la industria química, el consumo de energías primarias representa aproximadamente el $40 \%$ del costo total de operación de la planta (Zargarzadeh y col., 2007), esta energía es usada en forma de servicios auxiliares para que las corrientes de proceso alcancen las condiciones de presión y temperatura que requiera el proceso para transformar la materia prima. Las redes de intercambio de calor representan un elemento clave en el diseño de plantas químicas, pues permiten llevar a cabo la integración térmica entre las corrientes de proceso como un sistema independiente, permitiendo reducir los costos de operación de un proceso. 
La síntesis de redes de intercambio de calor consiste en el desarrollo de metodologías sistemáticas que permiten definir una estructura lógica para la colocación de intercambiadores de calor y servicios auxiliares necesarios, para llevar a cabo una tarea óptima de recuperación de calor. Por otro lado, diseñar redes de intercambio de calor económicas es una tarea desafiante ya que el problema de síntesis se caracteriza por ser altamente combinatorio, no lineal y no convexo, incrementando el grado de dificultad de forma exponencial conforme el número de corrientes de proceso es mayor; un proceso industrial típico presenta entre 30 y 80 corrientes de proceso (Gundersen y Naess, 1988), lo que implica que la mayoría de las formulaciones actuales resultan incapaces de resolver este tipo de problemas.

Furman y Sahinidis (2001) proporcionan una demostración formal que clasifica a la síntesis de redes de intercambio de calor en un subconjunto de problemas de optimización particularmente complejos conocidos como "No Polinomiales-Difíciles en el sentido fuerte" para los cuales, de acuerdo con la teoría de complejidad computacional, se puede afirmar que es imposible formular algoritmos que resuelvan el problema en tiempos eficientes.

A pesar de las dificultades descritas y debido a los beneficios significativos que conlleva el diseño de redes de intercambio de calor óptimas, se han realizado progresos considerables que han mantenido a la síntesis de redes de intercambio de calor como un área de investigación activa durante las últimas cuatro décadas, convirtiéndose en uno de los problemas más relevantes dentro de la ingeniería de sistemas de proceso. Por estas razones, diversas metodologías de diseño y optimización han sido propuestas en la literatura para encarar los desafíos que supone el problema de síntesis. Revisiones extensas han sido realizadas examinando los avances en el tema por Gundersen y Naess (1988), Jeżowski (1994a, 1994b) y Furman y Sahinidis (2002).

Ten Broeck (1944) desarrolló el primer trabajo relacionado con la síntesis de redes de intercambio de calor, y fueron posteriormente Masso y Rudd (1969) quienes definieron formalmente el problema. Los trabajos de Westbrook (1961), Hwa (1965) y Kesler y Parker (1969) fueron algunos de los precursores en abordar el problema de síntesis desde una perspectiva de modelado matemático, sin embargo, no fue hasta que se estableció la metodología de punto de pliegue (Linnhoff y Hindmarsh, 1983) que la industria y la academia se interesaron fuertemente en el problema de síntesis y se realizaron los primeros avances importantes en el tema. La metodología de punto de pliegue constituye una piedra angular para la comprensión del 
diseño de redes de intercambio de calor. Esta herramienta basada en principios heurísticos y termodinámicos permite calcular de manera sistemática, previamente al diseño de una red de intercambio de calor, las metas para el uso mínimo de servicios auxiliares, así como las metas de área que caracterizan al sistema de recuperación de calor permitiendo estimar los costos que deben presentar las redes de intercambio de calor a diseñar. Además, mediante el uso de reglas heurísticas simples, es posible determinar diseños de redes de intercambio de calor factibles.

Aunque la metodología de punto de pliegue es una valiosa herramienta que se ha mantenido como un tema de investigación de gran de interés (Tan y col., 2014, Gadalla, 2015), los cálculos que se requieren usualmente resultan en una tarea tediosa que no necesariamente concluyen en redes de intercambio de calor óptimas, generando diseños que pudieran ser poco atractivos en términos económicos. Estas desventajas han impulsado el desarrollo de metodologías más rigurosas que reduzcan los cálculos realizados a mano, haciendo uso de algoritmos y herramientas de programación matemática que faciliten la tarea de síntesis e incrementen las posibilidades de generar redes de intercambio de calor de bajo costo con una integración energética eficiente.

La mayoría de las metodologías de programación matemática para la síntesis de redes de intercambio de calor desarrolladas en la literatura pueden ser divididas en dos grupos: las metodologías secuenciales y las metodologías simultáneas. Ambos enfoques tienen la característica de que en su mayoría recurren a modelos de programación entera mixta (PEM), incorporando variables binarias que generan los apareamientos entre las corrientes de proceso. Estos planteamientos suelen significar un crecimiento exponencial en la complejidad del problema de síntesis conforme el número de corrientes incrementa, por lo que problemas de mediana y gran suelen representar un impedimento para formulaciones de esta naturaleza.

\subsubsection{Metodologías secuenciales}

Las metodologías secuenciales surgen debido a que las herramientas computacionales en un principio no eran suficientes para resolver modelos complejos de programación matemática (Verheyen y Zhang, 2006), este enfoque propone descomponer el problema de síntesis en sub- 
problemas que son computacionalmente más simples. Estas metodologías poseen la característica de utilizar una perspectiva física para el diseño de redes de intercambio de calor, resolviendo gradualmente los sub-problemas necesarios para obtener una estructura de red factible, iniciando por el que supone un mayor impacto en el costo del diseño final.

Biegler y col. (1997) enumeran los tres sub-problemas que usualmente se resuelven para la síntesis de redes de intercambio de calor utilizando el esquema secuencial. En primer lugar, mediante un modelo de programación lineal (PL) se determina el requerimiento mínimo de servicios auxiliares, posteriormente mediante un modelo de programación lineal entera mixta (PL-EM) se fija el número mínimo de apareamientos entre corrientes de proceso para la integración de calor y por último se plantea minimizar el costo total anual (CTA) de una estructura mediante un modelo de programación no lineal (PNL). La desventaja de este tipo de aproximaciones recae en la imposibilidad de asegurar soluciones óptimas, puesto que un número considerable de soluciones se descartan conforme se imponen las restricciones de cada subproblema.

Cerdá y col. (1983) formularon el problema de uso mínimo de servicios auxiliares como un modelo de PL usando el modelo de transporte, esta metodología consiste en dividir las corrientes de proceso en intervalos de temperatura, habilitando todas las posibles rutas directas para la transferencia o "transporte" de energía desde las corrientes de proceso calientes a las corrientes frías. Con el mismo enfoque Cerdá y Westerberg (1983) desarrollaron un modelo de programación lineal entera mixta (PL-EM) para la determinación del número mínimo de apareamientos necesarios para la síntesis de una red de intercambio de calor representados por las variables binarias activas.

Con una visión similar, Papoulias y Grossmann (1983) determinaron metas de energía y el mínimo número de apareamientos entre corrientes de proceso con modelos de PL y PL-EM respectivamente basados en el modelo de transbordo. Esta es una variación simplificada del modelo de transporte que plantea el mismo objetivo de distribución óptima de recursos, con la diferencia de que la energía se transporta de forma indirecta de las corrientes calientes a nodos intermedios representados por intervalos de temperatura y posteriormente a las corrientes frías, reduciendo considerablemente el número de variables con relación al modelo de transporte. 
Los apareamientos entre corrientes de proceso determinados por el modelo de transporte o transbordo brindan elementos suficientes para diseñar redes de intercambio de calor, no obstante, decisiones a consideración del diseñador deben ser tomadas en cuenta para la colocación de los equipos, así como para definir si es necesario usar divisores y mezcladores en las corrientes de proceso, introduciendo incertidumbre acerca de si la red final representa la mejor opción disponible. Floudas y col. (1986) partiendo de la solución del modelo de transbordo, desarrollaron un modelo de programación no lineal (PNL) que minimiza el costo total anual (CTA) y automatiza la generación de configuraciones de red. La estrategia consiste en derivar sub-redes dependiendo de los puntos de pliegue y generando una superestructura para cada posible apareamiento en la red. La solución del modelo indica las interconexiones que se deben conservar, para la obtención de una red de intercambio con CTA óptimo.

Gundersen y col. (1997) implementaron un enfoque secuencial que intenta hacer frente a problemas de escala industrial. Esta metodología de carácter iterativo permite diseñar redes cercanas al óptimo, otorgando cierta interacción con el usuario y buscando realizar un balance adecuado entre el costo de la red, la complejidad, operabilidad y controlabilidad de la misma. El enfoque secuencial propuesto por estos autores está basado en la premisa de elegir correctamente la selección de apareamientos entre corrientes de proceso. La metodología inicia preoptimizando el nivel de recuperación de energía dado por la diferencia mínima de temperaturas para la recuperación de calor (HRAT) y obteniendo el número mínimo de apareamientos con un modelo de transbordo, posteriormente utilizan un modelo vertical de programación lineal entera mixta (PL-EM) para obtener una distribución de cargas térmicas. Una vez obtenida la distribución de cargas térmicas se optimiza con un modelo de PNL (Floudas y col., 1986) para obtener la estructura final de la red. Cabe mencionar que es posible reajustar el nivel de recuperación de energía y el número de unidades, dando libertad al usuario y permitiendo realizar iteraciones hasta encontrar una solución satisfactoria. Posteriormente Hashemi-Ahmady y col. (1999) realizan avances significativos en el modelo de PNL para el enfoque secuencial, agregando una relajación convexa a los balances de calor en los intercambiadores (cotas inferiores) y buscando determinar el óptimo global del modelo. La búsqueda del óptimo global consiste en dos etapas, primero se resuelve el modelo de PNL con varios puntos de arranque (cotas superiores) y posteriormente se realiza una búsqueda determinista rigurosa para garantizar la optimalidad global del diseño de red. 
Bajo la misma línea de investigación Anantharaman y Gundersen (2006) propusieron un método de recorte modal para explorar la obtención del óptimo global, el cual consiste de una primera fase para encontrar una solución óptima local y obtener una solución cuasi-óptima global, y una segunda fase para encontrar una solución factible con el valor de la función objetivo que iguale a la del cuasi-óptimo global y obtener un punto de partida para encontrar una mejor solución óptima local. Si no se encuentra una solución factible tras repetir los pasos, el cuasi-óptimo global es tomado como la solución óptima global.

Zhu (1997) realiza una descomposición del problema de síntesis en una serie de bloques basándose en la idea de transferencia de calor vertical, donde las curvas compuestas pueden combinarse entre sí cuando presentan perfiles similares para formar un intervalo mayor o bloque. El autor define una etapa de metas, donde es posible explotar la diferencia de los coeficientes de transferencia de calor utilizando técnicas de punto de pliegue, y una etapa de diseño donde se consideran los efectos de las temperaturas y cargas térmicas usando técnicas de programación matemática. Los apareamientos se construyen sobre curvas cuasi-compuestas que son una aproximación en línea recta de las curvas compuestas reales a lo largo de un bloque. La selección del número mínimo de apareamientos se resuelve con un modelo de PL-EM, posterior a esto se determinan las corrientes en cada bloque que deben ser divididas considerando todas las posibilidades de apareamiento que involucran división de corrientes y resolviendo un modelo de PNL-EM. Por último, las soluciones obtenidas son optimizadas con el fin de disminuir los costos.

Shethna y col. (2000) propusieron un modelo de transporte usando PL-EM basado en intervalos de temperaturas para predecir metas de servicios auxiliares y la distribución de cargas térmicas, lo que se traduce en un balance adecuado entre costos de capital y operación de la red final. El modelo toma en cuenta múltiples servicios auxiliares, en donde cada servicio es una variable de optimización. Se realizan aproximaciones lineales de la función costos y de la diferencia media logarítmica, convirtiéndose en un problema de programación lineal, por lo que es posible lidiar con problemas que incluyen un número considerable de corrientes. Después de obtener una distribución de cargas térmicas, se puede sintetizar una red de intercambio de calor cercana al óptimo donde el diseñador debe tomar decisiones acerca de la colocación de equipos. 
Pettersson (2005) propone un enfoque secuencial de reducción de apareamientos para la síntesis de redes de intercambio de calor de escala industrial. Esta metodología descompone el problema de síntesis resolviendo cuatro sub-problemas con la misma meta, pero cambiando el grado de relajación, en lugar de resolver metas secuenciales como en los métodos clásicos. El primer paso consiste en un modelo de transporte que minimiza únicamente los costos de área por intercambiador de calor y los costos de servicios auxiliares omitiendo los costos unitarios. En el paso 2 se incluyen los costos unitarios en la función objetivo introduciendo una variable binaria por cada apareamiento, esto se traduce en un modelo de PL-EM. En el tercer paso se identifican posibles sub-conjuntos en donde las corrientes no están interconectadas en la red final, otorgando la capacidad de realizar la síntesis en cada sub-conjunto de manera independiente logrando reducir la complejidad del paso 4. Si no es posible identificar sub-conjuntos se define un número máximo de apareamientos en cada sub-conjunto, eliminándolos del conjunto considerado. Lo anterior resulta en grupos de corrientes definidos, así como en los apareamientos pertenecientes a cada conjunto. El cuarto paso es la etapa de diseño, donde los sub-conjuntos del sub-problema de agrupación se implementan en una formulación de PL-EM y los grupos se diseñan por separado.

Resolver el sub-problema de mínimo número de apareamientos (PL-EM) en problemas de gran escala representa el principal obstáculo para los enfoques secuenciales debido a la explosión combinatoria que exhiben. Anantharaman y col. (2010) plantean una modificación y una reformulación del modelo de transbordo de Papoulias y Grossmann (1983) para aminorar las dificultades numéricas del problema. La modificación consiste en la relajación de programación lineal (PL) empleando una restricción más justa derivada del trabajo de Zamora y Grossmann (1997), para la máxima cantidad de calor intercambiado entre dos corrientes usando una relación basada en información termodinámica. Los autores también investigan reducir la diferencia entre la relajación PL y la solución binaria real usando cortes enteros, obligando a que al menos una corriente caliente de proceso o de servicio auxiliar caliente intercambie calor con cada corriente de proceso fría (de manera análoga para las corrientes calientes), y condicionan el número mínimo de apareamientos por corriente. Por otro lado, en este trabajo se propone una reformulación parcial del problema del mínimo número de apareamientos haciendo uso de elementos de partición de conjuntos, definiendo nuevas variables binarias para cada conjunto de posibles apareamientos recurriendo a restricciones termodinámicas y heurísticas para reducir el tamaño del problema. 
Chen y col. (2015) también enfrentan las dificultades del problema del número mínimo de unidades proponiendo modelos de transbordo de PL-EM con el fin de resolver problemas de gran escala en tiempos razonables. Las diferentes propuestas consisten en reformulaciones del modelo de transbordo que incluyen desagregación del modelo, así como la adición de cortes enteros para fortalecer la relajación de PL y reducir el tiempo de solución. Otras estrategias que buscan mantener la rigurosidad en la determinación del óptimo global son investigadas en este trabajo, como la implementación de mejoras en el método de ramificación y acotamiento imponiendo prioridades de ramificación, y utilizando programación en paralelo. No obstante, estas formulaciones pudieran exceder el criterio de tiempos aceptables en problemas de gran escala para lo que los autores proponen esquemas aproximados tratando de determinar buenas soluciones y, en el mejor de los casos soluciones óptimas globales, en tiempos cortos.

Recientemente Pouransari y Maréchal (2015) desarrollaron una metodología para la síntesis de redes de intercambio de calor de gran escala mediante una descomposición del problema. Haciendo uso de un modelo de PL-EM para resolver el problema de requerimiento mínimo de servicios auxiliares. Las corrientes de proceso se agrupan formando subsistemas, donde decisiones deben ser tomadas basadas en restricciones de planta $\mathrm{u}$ otros factores, para evitar apareamientos no deseados. Cada conjunto de corrientes se "empaca" y se reemplaza por una corriente virtual fría y una caliente. Un modelo de PL-EM es usado para generar la distribución de cargas térmicas y minimizar el número de conexiones entre corrientes virtuales y servicios auxiliares. Para asegurar un número mínimo de conexiones se añaden apareamientos forzados entre corrientes virtuales del mismo subsistema, posteriormente se desempacan los subsistemas uno por uno considerando los apareamientos prohibidos y forzados como restricciones del problema. Si todos los subsistemas se desempacan, a partir de la distribución de cargas térmicas, es posible generar una red de intercambio de calor manualmente o utilizando un modelo de programación no lineal.

\subsubsection{Metodologías simultáneas}

En contraste con los enfoques secuenciales, las metodologías simultáneas, tienen la capacidad de resolver el problema de síntesis de redes de intercambio de calor en un solo paso, buscando 
realizar un balance adecuado entre la energía recuperada, número de equipos y CTA de la red. Lo anterior ha convertido a este tipo de estrategias en uno de los principales paradigmas de programación en el campo de investigación durante los últimos años.

Estas metodologías suelen ser representadas por una superestructura que incluye variables continuas y discretas, conteniendo un número considerable de configuraciones que usualmente se presentan en las redes de intercambio de calor. El enfoque de solución definiendo los valores óptimos de las variables de decisión y la configuración de la red de intercambio de calor de forma simultánea. Sin embargo, las dificultades numéricas que surgen de estas metodologías las hacen adecuadas únicamente para lidiar con problemas de pequeña y mediana escala, donde en la mayoría de los casos se requiere hacer uso de simplificaciones y suposiciones para que los modelos generen soluciones óptimas en tiempos razonables.

Ciric y Floudas (1991) desarrollaron una formulación simultánea basada en una estructura de red generalizada llamada hiperestructura (Floudas y col., 1989) para la determinación de una configuración óptima de red, y un modelo de transbordo modificado (Papoulias y Grossmann, 1983) para la selección cargas térmicas. La hiperestructura se formula como un modelo de PNLEM sobre los intervalos de temperatura por lo que se requiere definir un valor para el nivel de recuperación de energía.

Probablemente el enfoque simultáneo más recurrente en la síntesis de redes de intercambio de calor es el desarrollado por Yee y Grossmann (1990). El modelo denominado Synheat consiste en una representación de superestructura por etapas que incluye divisores y mezcladores en cada etapa permitiendo que cada corriente caliente pueda intercambiar calor con todas las corrientes frías. El modelo Synheat es relativamente fácil de implementar pues posee una región factible definida por restricciones lineales cuya solución proporciona la estructura de red, área de intercambio de calor y requerimientos de servicios auxiliares óptimos de forma simultánea, con las limitaciones de asumir mezclado isotérmico y un solo divisor por corriente en cada etapa. Sumado a esto, las implicaciones numéricas que genera la formulación de PNL-EM del modelo Synheat dificultan la determinación del óptimo global y en problemas de mediana escala, se produce la explosión combinatoria por el crecimiento exponencial del tamaño del espacio de búsqueda. Lo anterior ha motivado a diferentes autores a extender el modelo, así como proponer 
distintas suposiciones que permitan obtener soluciones óptimas disminuyendo, dentro de lo posible, la inversión de recursos computacionales.

Zamora y Grossmann (1998a) abordan la optimización global determinista del modelo Synheat sin divisiones de corrientes, utilizando un algoritmo de búsqueda híbrido de ramificación y acotamiento con aproximaciones exteriores, haciendo uso de sub-estimadores convexos para el área de intercambio de calor y asumiendo la diferencia media aritmética de temperaturas (DMAT) como una aproximación suficiente para la diferencia media logarítmica de temperaturas (DMLT). Esto se traduce en un modelo de PNL-EM que predice cotas justas al óptimo global. Zamora y Grossmann (1997) en un trabajo posterior, implementan en el modelo Synheat sin división de corrientes un conjunto de planos que acotan la DMLT por arriba, lo que supone un modelo de PNL-EM linealmente restringido, el cual se resuelve con un método de ramificación y acotamiento para predecir cotas inferiores rigurosas del costo total anual de la red. El modelo de PNL-EM genera diseños prometedores que son posteriormente optimizados para la determinación del óptimo global mediante un modelo de PNL.

Björk y Westerlund (2002) evaluaron la posibilidad de extender el modelo Synheat con división de corrientes y eliminando la suposición de mezclado isotérmico, en este trabajo se plantea resolver sub-problemas convexificados en lugar de los procedimientos de ramificación para asegurar la obtención de la solución óptima global. Bergamini y col. (2007) por otro lado, aborda la optimización global determinista del modelo Synheat con un algoritmo de aproximaciones exteriores desarrollando subestimadores para los términos no convexos utilizando una perspectiva física. Pettersson (2008) propone el uso de la diferencia media geométrica de temperaturas (DMGT) para la DMLT y una linealización por partes para asegurar optimalidad global.

Huang y col. (2012) plantean una superestructura por etapas modificada basada en el modelo Synheat con mezclado no isotérmico. Los autores proponen cotas mejoradas para las temperaturas de las sub-corrientes, así como restricciones lógicas para eliminar combinaciones redundantes en la superestructura y reducir el tiempo de cómputo.

Li y col. (2012) proponen un enfoque aumentado de síntesis de redes de intercambio de calor, introduciendo costos al modelo Synheat para todos los divisores y mezcladores, así como la inclusión de capacidades caloríficas calculadas a partir de una relación dependiente de la 
temperatura. Los autores plantean una etapa de interacción con el usuario que permite cancelar apareamientos que sean indeseados bajo algún criterio ingenieril o termodinámico, y añaden un procedimiento de una sola etapa utilizando algoritmos genéticos para optimizar el consumo de servicios auxiliares y el costo de inversión de forma simultánea para evitar caer soluciones óptimas locales.

Otras metodologías simultáneas han sido propuestas en búsqueda de soluciones que no pueden ser determinadas por el modelo Synheat e intentando sobrepasar las limitaciones que este modelo presenta. Huang y Karimi (2013) proponen dos superestructuras nuevas para la síntesis de redes de intercambio de calor, utilizando un enfoque por etapas centrada en apareamientos, y un enfoque sin etapas centrada en intercambiadores de calor. Ambas estructuras permiten flujo cruzado, apareamientos cíclicos y equipos en serie sobre una sub-corriente, sin embargo, incluir estas características también implica un gasto considerable en términos de recursos computacionales.

Recientemente Hong y col. (2017) desarrollaron una variación del modelo de transbordo formulado como un modelo de PNL-EM con restricciones lineales. El modelo incluye divisiones de corrientes, baipás, mezclado no isotérmico, recirculación y la posibilidad de múltiples servicios auxiliares. Esta metodología de síntesis permite definir diseños de redes de intercambio de calor en un solo paso considerando la distribución de cargas térmicas y tomando en cuenta un patrón de flujo másico.

Uno de los beneficios que implica el diseño de redes de intercambio de calor óptimas es la posible disminución del uso de energía externa en plantas industriales, lo que aporta de forma positiva al problema medioambiental al que la sociedad actual se enfrenta, adicionalmente formular metodologías que aporten avances a la solución del problema de síntesis, representa la oportunidad de implementar reducciones significativas en el costo total de un proceso. No obstante, debido a la naturaleza combinatoria del problema de síntesis y a las dificultades que esto representa, la industria y la academia continúan destinando esfuerzos para desarrollar diferentes métodos que permitan determinar diseños de redes de intercambio de calor de bajo costo en tiempos razonables, incluso en problemas de escala industrial.

En este trabajo se desarrolla una metodología que recurre a la descomposición del problema de síntesis por lo que se puede clasificar como un enfoque secuencial. La estrategia propuesta 
representa una alternativa para el diseño de redes de intercambio de calor buscando evitar las dificultades numéricas que surgen en la mayoría de las metodologías de síntesis secuenciales clásicas. A continuación, se plantea el enunciado del problema, así como los objetivos formales de la tesis.

\subsection{Enunciado del problema}

Formalmente el problema de síntesis de redes de intercambio de calor abordado en este trabajo se formula a continuación. Se cuenta con un conjunto de corrientes calientes de proceso que deben ser enfriadas y un conjunto de corrientes de proceso frías que deben ser calentadas. Asociado a cada corriente de proceso existen valores conocidos de flujos de capacidad calorífica, así como de temperaturas de suministro y objetivo. También se cuenta con conjunto de servicios de calentamiento y enfriamiento están disponibles, con valores conocidos de temperaturas de entrada y salida, y sus correspondientes costos. El propósito del problema consiste en sintetizar una red de intercambio de calor que cumpla con las especificaciones de los datos dados al menor costo total anual posible.

Se tienen en cuenta los siguientes suposiciones: i) los flujos de capacidad calorífica se consideran constantes, calculados a una temperatura intermedia; ii) las corrientes de proceso no experimentan cambios de fase; iii) se utilizan intercambiadores de calor de contracorriente; iv) los coeficientes de película para la transferencia de calor para todas las corrientes o los coeficientes globales de transferencia de calor para todos los intercambiadores son conocidos; v) se especifica una diferencia de temperatura mínima EMAT para el intercambio de calor; vi) se conocen los costos unitarios de operación, para servicios de calentamiento y enfriamiento; y vii) los costos de capital de los intercambiadores de calor individuales siguen una ley de costos exponencial en función del área de transferencia de calor. 


\subsection{Objetivos del proyecto}

\subsubsection{Objetivo general}

La investigación realizada en esta tesis se centraliza en proponer algoritmos y hacer uso de herramientas de programación matemática para llevar a cabo la tarea síntesis de redes de intercambio de calor. Puesto que las principales complicaciones del diseño de redes de intercambio de calor óptimas emergen de las dificultades numéricas que las formulaciones PLEM y PNL-EM conllevan (Furman y Sahinidis, 2002), se investiga la posibilidad de implementar un enfoque de síntesis secuencial el cual no requiere del cálculo del número mínimo de apareamientos, prescindiendo del uso de variables binarias.

La propuesta consiste en definir un número considerable de apareamientos factibles entre corrientes de proceso que pueden ser interpretados como un conjunto sobreestimado de intercambiadores de calor. Mediante una estrategia que automatiza la colocación de equipos en serie y en paralelo sobre diferentes ramales de sub-corrientes de proceso según sea requerido, es posible definir diseños redundantes de red que, al tener topologías preestablecidas, acotan la región factible del problema y presentan un número considerable de grados de libertad. La obtención de redes de intercambio de calor finales se produce al optimizar los diseños base determinando los equipos y divisiones de corriente que deben de ser eliminados para obtener los mejores diseños óptimos simplificados contenidos en la estructura redundante. Una de las principales virtudes del método consiste en la posibilidad de generar y optimizar de forma sistemática un conjunto de topologías redundantes, donde cada una puede contener diferentes

diseños óptimos incrementando las posibilidades de obtener soluciones de bajo costo y permitiendo al usuario definir el tiempo de cómputo se desea invertir fijando un cierto número de diseños base a evaluar. 


\subsubsection{Objetivos particulares}

1. Determinar mediante el uso de un modelo de transporte (modelo de LP) intercambios de calor factibles para la tarea de recuperación de calor, y que a su vez permitan definir estructuras con un número considerable de grados de libertad.

2. Optimizar el CTA de las redes generadas mediante técnicas de PNL eliminando los equipos sobreestimados con el propósito de mantener el balance adecuado entre recuperación de calor y distribución de área.

3. Desarrollar una metodología sistemática que descomponga la tarea de síntesis en los subproblemas de generación y optimización de múltiples de redes intercambio redundantes, basados en planteamientos de programación matemática de PL y PNL respectivamente para la obtención de diseños óptimos.

4. Evaluar el alcance de la metodología propuesta utilizando diferentes casos de estudio de la literatura haciendo uso de resolvedores comerciales de optimización.

\subsection{Contenido del trabajo}

Este trabajo involucra seis capítulos. Una breve introducción y reseña de los trabajos que abordan la síntesis de redes de intercambio de calor se detalla en el Capítulo 1.

En el Capítulo 2 se remarca la posibilidad de usar el modelo de transporte para resolver el problema de uso mínimo de servicios auxiliares (Cerdá y col., 1983) como un medio para generar un número sobrado de apareamientos factibles entre corrientes de proceso.

El Capítulo 3 consiste en una descripción del algoritmo de multi-arranque estocástico en dos fases (Núñez-Serna y Zamora, 2016) para la optimización de diseños base con topología preestablecida. Este método permite minimizar el CTA de una red de intercambio de calor y llevar a cabo reducciones estructurales en caso de ser necesario. El enfoque implementa una técnica para evaluar diferentes inicializaciones pseudo-aleatorias de las variables de decisión, realizando una búsqueda en diferentes zonas de la región factible para determinar múltiples soluciones óptimas locales. 
El Capítulo 4 corresponde al aporte principal de este trabajo, la generación de topologías redundantes para la síntesis de redes de intercambio de calor. Se proponen diferentes estrategias para interpretar la solución del modelo de transporte del Capítulo 2 como redes de intercambio de calor sobreestimadas. También se plantea la posibilidad de generar una variedad de estructuras a optimizar mediante la variación del parámetro HRAT. Estas estructuras se acoplan a la estrategia de optimización del Capítulo 3 para determinar diseños de red óptimos.

En el Capítulo 5 se presentan los resultados de la aplicación de la metodología de síntesis a siete casos de estudio de la literatura, mostrando la posibilidad de generar diseños con costos competitivos y en el mejor de los casos, de obtener soluciones con configuraciones que presentan un menor costo en comparación con los reportados en la literatura.

Finalmente, las conclusiones y posibles líneas de investigación para una futura implementación del método se presentan en el Capítulo 6. 


\title{
Capítulo 2. Modelo de Transporte
}

\author{
Notación \\ Índices \\ $i \quad$ Índice de corriente de proceso caliente \\ $j \quad$ Índice de corriente de proceso fría \\ $l \quad$ Índice de intervalo de temperatura para corriente de proceso caliente \\ $l 1 \quad$ Índice de intervalo de temperatura para corriente de proceso fría
}

\section{Conjuntos}

$I$

$J$

$L$

$L 1$

\section{Parámetros}

HRAT Diferencia de temperaturas para la recuperación de calor $\left({ }^{\circ} \mathrm{C}\right)$

$\mathrm{TS}_{\mathrm{i}}, \mathrm{TS}_{\mathrm{j}} \quad$ Temperaturas de suministro de corrientes de proceso $\left({ }^{\circ} \mathrm{C}\right)$

$\mathrm{TO}_{\mathrm{i}}, \mathrm{TO}_{\mathrm{j}} \quad$ Temperaturas objetivo de corrientes de proceso $\left({ }^{\circ} \mathrm{C}\right)$

$\mathrm{TH}_{1} \quad$ Temperatura en la frontera de un intervalo de temperatura caliente $\left({ }^{\circ} \mathrm{C}\right)$

$\mathrm{TC}_{11} \quad$ Temperatura en la frontera de un intervalo de temperatura fría $\left({ }^{\circ} \mathrm{C}\right)$

$\mathrm{HS}_{\mathrm{i}, 1} \quad$ Calor disponible de cada corriente caliente $i$ en el intervalo de temperatura $l(\mathrm{~kW})$

$\mathrm{HD}_{\mathrm{j}, 11} \quad$ Calor disponible de cada corriente fría $j$ en el intervalo de temperatura $l 1(\mathrm{~kW})$

$\mathrm{U}_{\mathrm{i}, \mathrm{j}}$ corriente fría $j(\mathrm{~kW})$ 


$\begin{array}{ll}\mathrm{NOL} & \text { Número total de intervalos de temperatura } \\ \mathrm{C}_{\mathrm{i}, \mathrm{l}, \mathrm{j}, 11} & \text { Costo de la recuperación } \\ \mathrm{CHU}_{\mathrm{j}, 11} & \text { Costo del calentamiento } \\ \mathrm{CCU}_{\mathrm{i}, 1} & \text { Costo del enfriamiento }\end{array}$

Variables continuas positivas

$q_{i, l, j, l 1} \quad$ Calor intercambiado entre una corriente caliente $i$ y una corriente fría $j(\mathrm{~kW})$

$q H U_{j, l 1} \quad$ Servicios de calentamiento requeridos en el intervalo de temperatura $l 1(\mathrm{~kW})$

$q C U_{i, l}, \quad$ Servicios de enfriamiento requeridos en el intervalo de temperatura $l(\mathrm{~kW})$

$y_{i, j} \quad$ corriente fría $j$

\subsection{Definición del modelo de transporte}

El modelo de transporte pertenece a una clase especial de problemas de programación lineal que conciernen a la optimización de redes. La primera formulación matemática formal fue propuesta por Hitchcock (1941) y posteriormente Dantzig (1951) lo formula como un problema de programación lineal desarrollando el primer algoritmo para resolverlo de forma eficiente aplicando el método Simplex. Actualmente se continúan realizado contribuciones en el tema, proponiendo diferentes métodos de solución (e.g. Ahmed y col., 2016, Juman y Hoque, 2015) debido a las diversas aplicaciones prácticas de los modelos de transporte o mejorando la eficiencia de los procedimientos ya propuestos para resolver problemas que involucran un importante número de restricciones.

El modelo de transporte se ocupa principalmente de problemas que buscan la forma en que un producto proveniente de diferentes orígenes de suministro es transportado a diferentes destinos, satisfaciendo plenamente los requisitos de demanda, considerando las restricciones de capacidad de producción, al menor costo posible. Hoy en día el modelo de transporte aún representa una herramienta útil para llevar a cabo tareas logísticas que requieran toma de decisiones en la 
asignación de recursos. En la Figura 2.1 se muestra una representación de red simple del modelo de transporte.

La formulación matemática del modelo de transporte requiere de los siguientes elementos.

- Existen $m$ fuentes denotadas como $\mathrm{S}_{\mathrm{i}} \mathrm{y} n$ destinos denotados $\mathrm{D}_{\mathrm{j}}$, cada uno representado por un nodo, donde $i=1, \ldots, m, j=1, \ldots, n$.

- Las líneas representan las rutas que vinculan la cantidad de bien transportado $x_{i, j}$ así como su costo unitario asociado $\mathrm{C}_{\mathrm{i}, \mathrm{j}}$ desde la $i$-ésima fuente al $j$-ésimo destino.

- Hay una cantidad disponible de bienes $a_{i}$ suministradas por el origen $S_{i}$.

- Existe una demanda de bienes $b_{i}$ requeridas por el destino $D_{j}$.

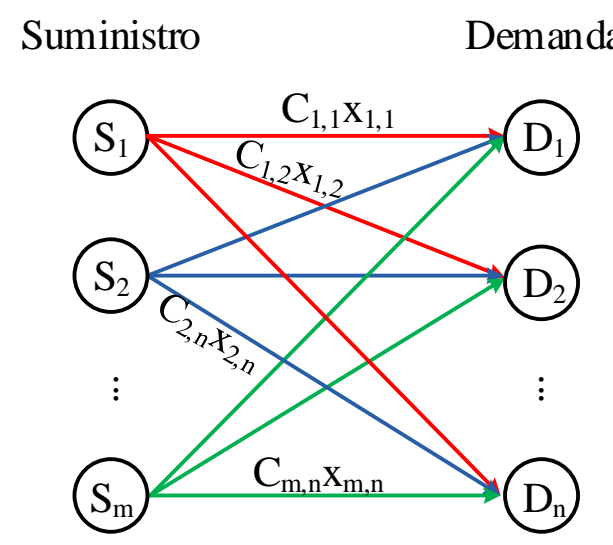

Figura 2.1. Representación de red del modelo de transporte.

El costo de transporte o función de transporte se puede escribir como.

$$
C T=\mathrm{C}_{1,1} x_{1,1}+\mathrm{C}_{1,2} x_{1,2}+\ldots+\mathrm{C}_{1, \mathrm{n}} x_{1, n}+\mathrm{C}_{2,1} x_{2,1}+\ldots+\mathrm{C}_{\mathrm{m}, 1} x_{m, 1}+\ldots+\mathrm{C}_{\mathrm{m}, \mathrm{n}} x_{m, n}
$$

Adicionalmente se debe satisfacer que todos los bienes disponibles en cada fuente pueden distribuirse a todos los puntos de demanda.

$$
\begin{gathered}
x_{1,1}+x_{1,2}+\ldots+x_{1, n} \leq \mathrm{a}_{1} \\
x_{2,1}+x_{2,2}+\ldots+x_{2, n} \leq \mathrm{a}_{2} \\
\vdots \\
x_{m, 1}+x_{m, 2}+\ldots+x_{m, n} \leq \mathrm{a}_{\mathrm{m}}
\end{gathered}
$$

Mientras que cada destino debe satisfacer sus necesidades de demanda. 


$$
\begin{gathered}
x_{1,1}+x_{2,1}+\ldots+x_{m, 1} \geq \mathrm{b}_{1} \\
x_{1,2}+x_{2,2}+\ldots+x_{m, 2} \geq \mathrm{b}_{2} \\
\vdots \\
x_{1, n}+x_{2, n}+\ldots+x_{m, n} \geq \mathrm{b}_{\mathrm{n}}
\end{gathered}
$$

El principal objetivo del modelo es determinar los valores óptimos de los bienes $x_{i, j}$ que deben ser transportados desde $\mathrm{S}_{i}$ hasta $\mathrm{D}_{j}$ para que el costo total del transporte sea mínimo, cumpliendo con las respectivas restricciones de suministro y demanda.

Dicho esto, el modelo de transporte se expresa como un problema de programación lineal de la siguiente forma.

Minimizar $\quad C T=\sum_{i=1}^{m} \sum_{j=1}^{n} \mathrm{C}_{i, j} x_{i, j}$

Sujeto a

$$
\begin{array}{lc}
\sum_{j=1}^{m} x_{i, j} \leq \mathrm{a}_{i} & i=1, \ldots, m \\
\sum_{i=1}^{n} x_{i, j} \geq \mathrm{b}_{j} & j=1, \ldots, n \\
C T, x_{i, j} \geq 0 & i=1, \ldots, m \quad \text { y } j=1, \ldots, n
\end{array}
$$

Sin pérdida de generalidad, es común asumir nodos ficticios para satisfacer completamente las restricciones de demanda y suministro, estos nodos balancean la diferencia entre el suministro y la demanda por lo que las desigualdades pueden ser escritas como ecuaciones. Cabe mencionar que dichos nodos deben tener asignado un costo de transporte unitario igual a cero, con el fin de preservar el costo total original.

Aceptando que se cumple que el total del suministro es igual al total de la demanda, $\sum_{i=1}^{m} \mathrm{~S}_{i}=\sum_{j=1}^{n} \mathrm{D}_{j}$ se puede escribir el modelo de transporte en su forma estándar.

\section{Minimizar}

$$
C T=\sum_{i=1}^{m} \sum_{j=1}^{n} \mathrm{C}_{i, j} x_{i, j}
$$


Sujeto a

$$
\begin{array}{ll}
\sum_{j=1}^{m} x_{i, j}=\mathrm{a}_{i} & i=1, \ldots, m \\
\sum_{i=1}^{n} x_{i, j}=\mathrm{b}_{j} & j=1, \ldots, n \\
x_{i, j} \geq 0 & i=1, \ldots, m \quad \text { y } j=1, \ldots, n
\end{array}
$$

El modelo de transporte tiene aplicación en diferentes campos, como son el diseño de procesos industriales, redes de comunicaciones, asignación de transporte, programación de maquinaria, optimización de horarios, entre otras. El modelo de transporte también ha sido empleado para la integración térmica de procesos y representa un elemento importante para el entendimiento y desarrollo de metodologías secuenciales para la síntesis de redes de intercambio de calor.

\subsection{Problema del requerimiento mínimo de servicios auxiliares}

Cerdá y col. (1983) formularon la determinación del consumo mínimo de servicios auxiliares como un problema de programación lineal basado en el modelo de transporte. En términos de recuperación de calor se define a las fuentes como las corrientes calientes y los servicios de calentamiento, y los destinos como las corrientes frías y los servicios de enfriamiento; se debe transportar (o transferir) la energía entre las corrientes de proceso y de servicios auxiliares,

minimizando el costo de transporte. Este sub-problema es el de mayor jerarquía en las metodologías secuenciales, debido a que el costo de operación asociado al sistema de servicios auxiliares suele ser el elemento dominante en costo total de un proceso (Papoulias y Grossmann, 1983).

El problema de mínimo requerimiento de servicios auxiliares se puede representar gráficamente sobre el diagrama de intervalos de temperatura (Flower y Linnhoff, 1978), considerando todas las posibles rutas de transferencia de calor entre corrientes calientes y frías. Con el fin de precisar la representación del problema del mínimo requerimiento de servicios auxiliares como un modelo de transporte de considera un ejemplo ilustrativo mostrado en la Tabla 2.1 cuyos datos de corrientes fueron extraídos de un trabajo de Zamora y Grossmann (1997). El problema consta 
de dos corrientes de proceso calientes y dos frías, así como con un servicio de calentamiento y un servicio de enfriamiento.

Tabla 2. 1. Datos de corrientes del Ejemplo ilustrativo 2.1.

\begin{tabular}{ccccc}
\hline Corriente & $\begin{array}{c}\text { Temperatura de } \\
\text { suministro } \\
\mathrm{Ts}\left({ }^{\circ} \mathrm{C}\right)\end{array}$ & $\begin{array}{c}\text { Temperatura } \\
\text { objetivo } \\
\mathrm{To}\left({ }^{\circ} \mathrm{C}\right)\end{array}$ & $\begin{array}{c}\text { Flujo de capacidad } \\
\text { calorífica } \\
\mathrm{F}\left(\mathrm{kW}^{\circ} \mathrm{C}^{-1}\right)\end{array}$ & $\begin{array}{c}\text { Coeficiente de } \\
\text { película } \\
\mathrm{h}\left(\mathrm{kW} \mathrm{m}^{-1}{ }^{\circ} \mathrm{C}^{-1}\right)\end{array}$ \\
\hline $\mathrm{H} 1$ & 180 & 75 & 30 & 0.15 \\
$\mathrm{H} 2$ & 240 & 60 & 40 & 0.10 \\
$\mathrm{C} 1$ & 40 & 230 & 35 & 0.20 \\
$\mathrm{C} 2$ & 120 & 300 & 20 & 0.10 \\
$\mathrm{HU}$ & 325 & 325 & - & 2 \\
$\mathrm{CU}$ & 25 & 40 & - & 0.50 \\
\hline
\end{tabular}

El rango total de temperaturas del problema es dividido definiendo una escala para las corrientes de proceso calientes y una escala para las corrientes de proceso frías. Los intervalos de temperatura se establecen donde se encuentra una temperatura de suministro u objetivo de alguna corriente de proceso. La diferencia de temperaturas para la recuperación de calor (HRAT) fija un desplazamiento relativo entre los perfiles de temperatura de corrientes de proceso y permite completar las escalas de temperaturas en la tabla de calor. Entonces, para un HRAT definido es posible determinar la meta de energía máxima a recuperar.

Un balance de energía puede ser llevado a cabo sobre cada intervalo donde una corriente de proceso está presente, estos intervalos están delimitados por fronteras y pueden ser vistos como los nodos del modelo de transporte, si NOL es el número total de intervalos de temperatura o nodos, entonces existen NOL+1 fronteras.

En la Figura 2.2 se describe la representación de red del modelo de transporte para la recuperación de calor valiéndose de los intervalos de temperatura. La Figura 2.2a describe todas las posibles rutas de intercambio de calor que la formulación del modelo de transporte puede generar con un valor de HRAT igual a $10{ }^{\circ} \mathrm{C}$, mientras que para Figura $2.2 \mathrm{~b}$ se fija el valor de HRAT en $15^{\circ} \mathrm{C}$. Es posible apreciar que la variación del parámetro HRAT determina un número diferente de intervalos de temperatura, así como la posición de las corrientes de proceso en el 
diagrama, lo que pudiera limitar o incrementar las posibles rutas de intercambios de calor que se traduce en una cantidad diferente de energía recuperada.

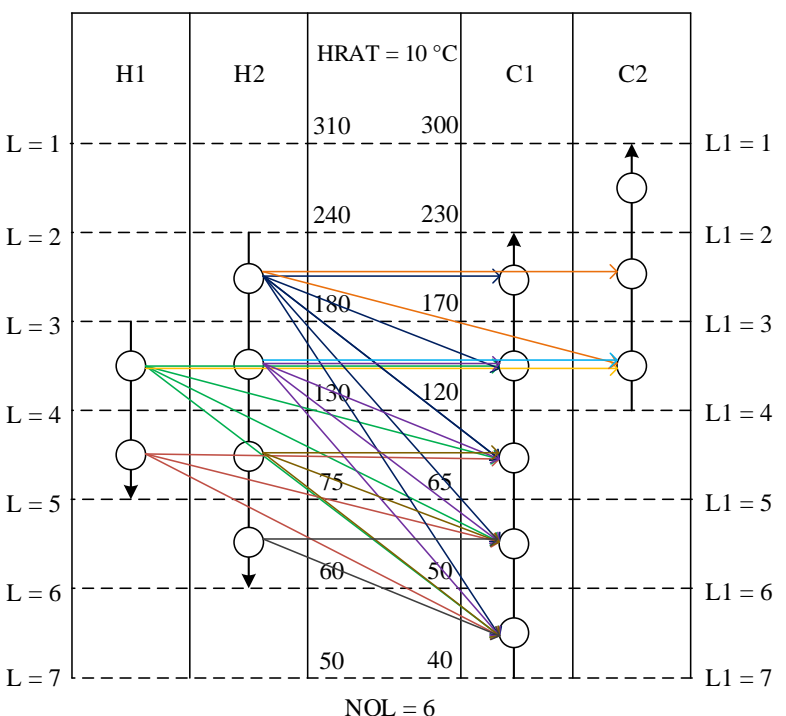

Figura 2.2a. HRAT $=10^{\circ} \mathrm{C}$

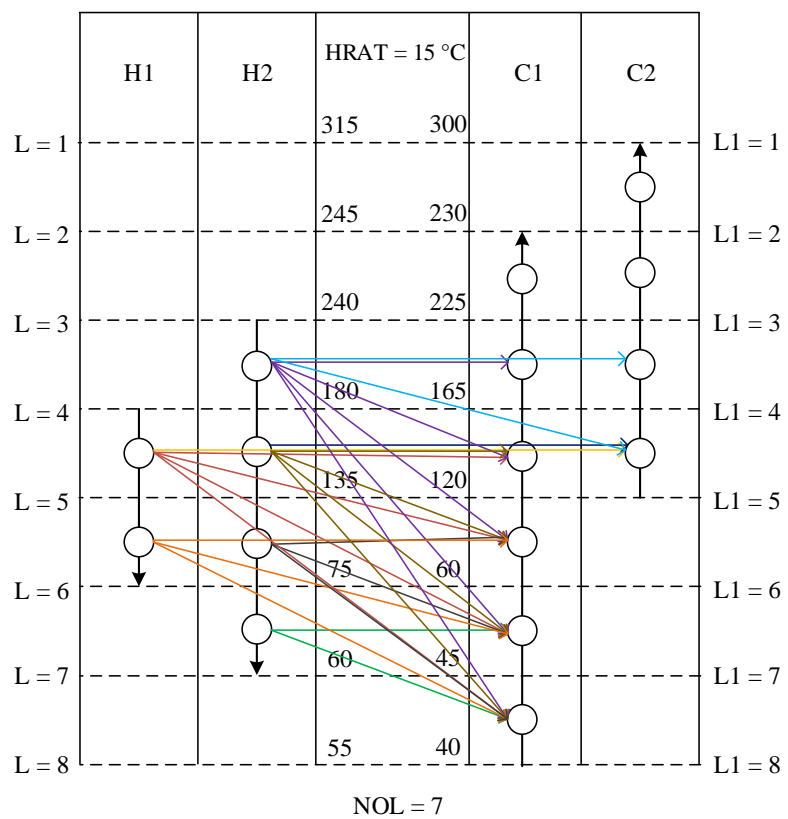

Figura 2.2b. HRAT $=15{ }^{\circ} \mathrm{C}$

Figura 2.2. Representación gráfica del modelo de transporte para el problema de uso mínimo de servicios auxiliares.

Para fines explicativos los servicios auxiliares no se incluyeron en la Figura 2.2. En la Figura 2.3 se ilustra de forma aislada los nodos de servicios auxiliares para un HRAT de $10{ }^{\circ} \mathrm{C}$. Cada nodo caliente tiene asociado un vínculo con el nodo de servicio de enfriamiento y cada nodo frío tiene asociado un vínculo con el nodo de servicio de calentamiento. Estos nodos cumplen la función de balancear el modelo de transporte en caso de que algunas corrientes de proceso posean un exceso o déficit de energía y requieran de servicios auxiliares para alcanzar las temperaturas objetivo establecidas por el proceso. Una representación completa del planteamiento del modelo de transporte para un HRAT de $10{ }^{\circ} \mathrm{C}$ se puede pensar como la unión de las Figuras 2.2a y 2.3.

Como se observa en la Figura 2.2 los posibles intercambios de calor entre corrientes de proceso solo están definidos desde algún nodo caliente hacia un nodo frío que se encuentra en el mismo intervalo de temperatura o hacia intervalos inferiores de menor temperatura. La segunda ley de la termodinámica impone que la dirección de la transferencia de calor solo puede fluir desde un cuerpo hacia otro con menor temperatura. Este principio garantiza la factibilidad del modelo al evitar cruces de temperaturas entre corrientes calientes y frías. Por otro lado, mediante 
restricciones similares también es posible prohibir apareamientos indeseados como son los casos en donde ciertas corrientes de proceso no pueden intercambiar calor debido a cuestiones de seguridad o por su posición geográfica en plantas reales.

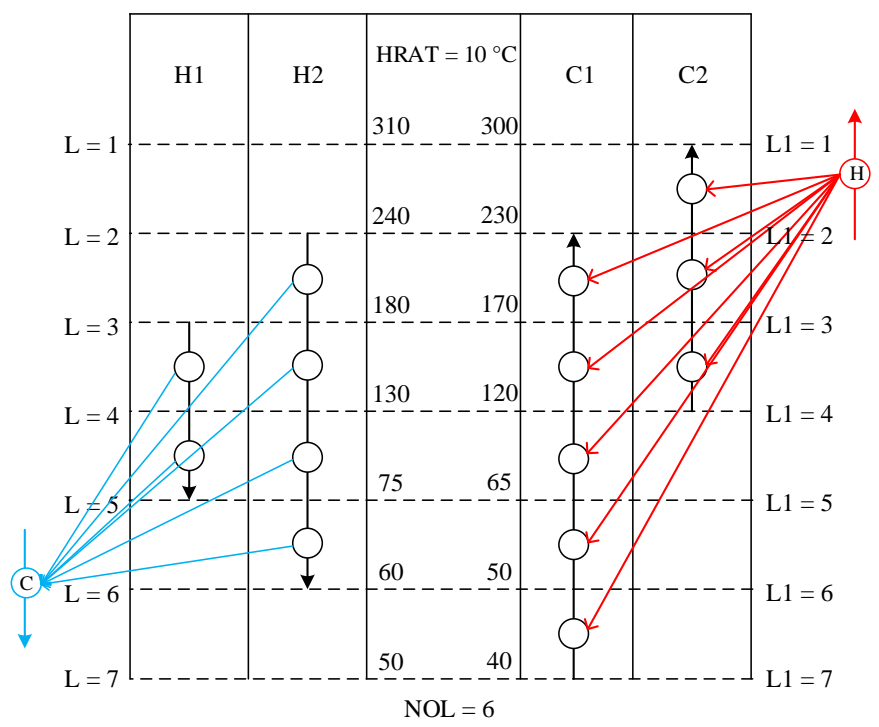

Figura 2.3. Representación de los nodos de servicios auxiliares para un HRAT igual a $10{ }^{\circ} \mathrm{C}$.

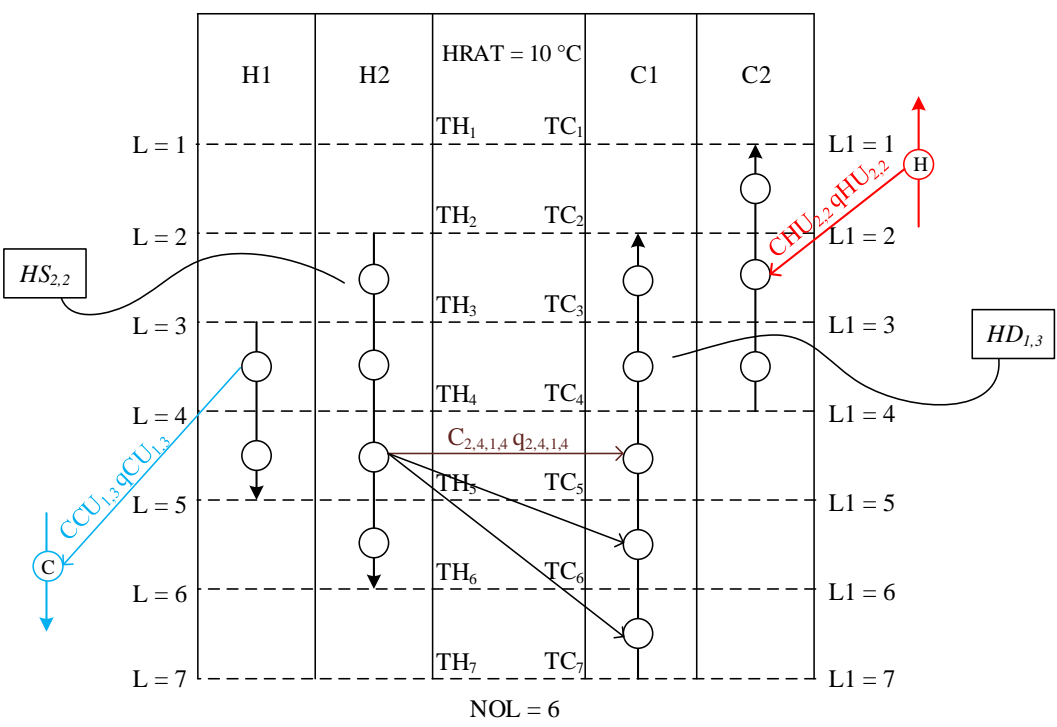

Figura 2.4. Ejemplos de notación sobre el diagrama de temperaturas para la formulación del modelo de transporte.

En la Figura 2.4 se especifican, sobre el diagrama de temperaturas, algunos elementos clave de la formulación del modelo de transporte para el problema de uso mínimo de servicios auxiliares con su respectiva notación. Estas nociones resultan de suma importancia para la comprensión del 
planteamiento del modelo de transporte cuya solución conforma una parte fundamental en el desarrollo de la metodología propuesta en este trabajo.

Con lo anterior se puede escribir el modelo de transporte como un modelo de PL cuya solución óptima indicará los intercambios de calor que deben llevarse a cabo para obtener un consumo mínimo de servicios auxiliares. Una característica destacable de los modelos de PL es que pueden ser resueltos en tiempos polinomiales, por lo que es posible lidiar con problemas que involucran varios millones de variables de forma eficiente, admitiendo para este tipo de formulaciones problemas de escala industrial con un requerimiento modesto de recursos computacionales.

$\operatorname{Min} \sum_{i \in I} \sum_{l \in L} \sum_{j \in J l 1 \in L 1} \sum_{i, l, j, l 1} q_{i, l, j, l 1}+\sum_{j \in J l 1 \in L 1} \mathrm{CHU}_{j, l 1} q H U_{j, l 1}+\sum_{i \in I} \sum_{l \in L} \mathrm{CCU}_{i, l} q C U_{i, l}$

Sujeto a

Restricciones de suministro.

$\sum_{j \in J} \sum_{l 1 \in L 1} q_{i, l, j, l 1}+q C U_{i, l}=\mathrm{HS}_{i, l} \quad i \in I, l \in L \backslash\{N O L+1\}$

Restricciones de demanda.

$\sum_{i \in I} \sum_{l \in L} q_{i, l, j, l 1}+q H U_{j, l 1}=\mathrm{HD}_{j, l 1} \quad j \in J, l 1 \in L 1 \backslash\{N O L+1\}$

Variables no negativas.

$q_{i, l, j, l 1}, q H U_{j, l 1}, q C U_{i, l} \geq 0 \quad i \in I, l \in L \backslash\{N O L+1\}, j \in J, l 1 \in L 1 \backslash\{N O L+1\}$

Donde:

$\mathrm{C}_{i, l, j, l 1}=M \quad \mathrm{Si} \quad l>l 1$

$\mathrm{C}_{i, l, j, l 1}=0 \quad \mathrm{Si} \quad l \leq l 1$

$\mathrm{CHU}_{j, l 1}=1$

$\mathrm{CCU}_{i, l}=1$ 
La primera ley de la termodinámica provee la ecuación de energía para calcular los cambios de entalpía en cada intervalo donde alguna corriente de proceso está presente.

Entalpías de las corrientes calientes.

$$
H S_{i, l}=F_{i}\left(T H_{l}-T H_{l+1}\right) \quad \text { si } \quad T O_{i}<T H_{l} \leq T S_{i} \quad i \in I, l \in L \backslash\{N O L+1\}
$$

Entalpías de las corrientes frías.

$$
H D_{j, l 1}=F_{j}\left(T H_{l 1}-T H_{l 1+1}\right) \quad \text { si } \quad T S_{j}<T C_{l 1} \leq T O_{j} \quad j \in J, l 1 \in L 1 \backslash\{N O L+1\}
$$

\subsection{Problema del número mínimo de apareamientos}

Cerdá y Westerberg (1983) formularon una representación analítica como un modelo de PL-EM para la síntesis de redes de intercambio de calor cuya solución permite determinar redes factibles con el número mínimo de apareamientos entre corrientes de proceso. La justificación para imponer redes de intercambio de calor con un número mínimo de equipos se debe a que en la mayoría de los casos cada intercambiador tiene asociado un cargo fijo, por lo que pudiera resultar evidente que disminuir el número de equipos implicará una reducción en los costos de capital de la red final. Sin embargo, debido a que el problema de síntesis de redes de intercambio de calor es altamente no lineal y a que no todas las funciones de costos poseen un cargo fijo, esta afirmación no es necesariamente cierta.

El problema de número mínimo de apareamientos representa el segundo sub-problema en las metodologías secuenciales. Es posible reformular el modelo de transporte como un problema de PL-EM introduciendo las variables binarias $y_{i, j}$ que pueden tomar los valores 1 y 0 dependiendo de la existencia de potenciales apareamientos que pueden ser asociados con un intercambiador de calor entre corrientes de proceso. La solución de este problema puede otorgar una o varias configuraciones de apareamientos que cumplan con la meta de número mínimo de apareamientos permitiendo generar posibles diseños de red con las mismas metas, pero con diferencias en el costo final de inversión.

El modelo de transporte formulado como problema de PL-EM para determinar el número mínimo de apareamientos se plantea de la siguiente forma. 
$\operatorname{Min} \sum_{i \in I} \sum_{j \in J} y_{i, j}$

Sujeto a

Restricciones de suministro.

$\sum_{j \in J l 1 \in L 1} \sum_{i, l, j, l 1}=\mathrm{HS}_{i, l} \quad i \in I, l \in L \backslash\{N O L+1\}$

Restricciones de demanda.

$\sum_{i \in I} \sum_{l \in L} q_{i, l, j, l 1}=\mathrm{HD}_{j, l 1} \quad j \in J, l 1 \in L 1 \backslash\{N O L+1\}$

Cotas para la transferencia de calor

$\sum_{l \in L l l \in L 1} \sum_{i, l, j, l 1} \leq \mathrm{U}_{i, j} y_{i, j} \quad i \in I, j \in J$

Variables no negativas.

$$
\begin{aligned}
& q_{i, l, j, l 1} \geq 0 \quad i \in I, l \in L \backslash\{N O L+1\}, j \in J, l 1 \in L 1 \backslash\{N O L+1\} \\
& y_{i, j} \in\{0,1\} \quad i \in I, j \in J
\end{aligned}
$$

Donde $U_{i, j}$ es una cota superior que indica la máxima cantidad de calor que puede ser intercambiada entre una corriente caliente y una corriente fría.

Este planteamiento debe cumplir con las metas de consumo mínimo de servicios auxiliares por lo que se debe fijar $\mathrm{HS}_{i, l} \mathrm{y}_{\mathrm{HD}_{j, l 1}}$ exactamente en los valores de servicios de calentamiento y enfriamiento obtenidos del sub-problema de uso mínimo de servicios auxiliares descrito en la Sección 2.2.

Con la solución de este problema las posibilidades de estructuras de red se reducen considerablemente, por lo que la tarea de síntesis de redes de intercambio de calor resulta menos compleja. Cerdá y Westerberg (1983) proponen una serie de pasos que permiten diseñar redes factibles utilizando los apareamientos obtenidos del modelo planteado en esta sección, sin embargo, el diseñador debe tomar decisiones acerca de la posición de equipos y de las posibles 
divisiones de corrientes, lo que pudiera conducir a soluciones que no necesariamente representan una opción económica.

Papoulias y Grossmann (1983) determinaron metas de uso mínimo de servicios auxiliares y para el número mínimo de apareamientos utilizando un modelo de transbordo bajo esquemas de PL y PL-EM, respectivamente. Este enfoque difiere del modelo de transporte al no transferir calor directamente desde intervalos de temperatura calientes hacia intervalos de temperatura fríos, en cambio las corrientes calientes transfieren el calor hacia nodos intermedios representados por intervalos de temperatura y posteriormente hacia las corrientes frías, donde el calor solo puede fluir desde un intervalo de temperatura superior hacia un intervalo de menor nivel de temperatura para asegurar la factibilidad termodinámica del problema. Esta formulación permite reducir considerablemente el tamaño del problema con respecto al modelo de transporte original, debido a que las únicas variables resultan ser los residuos de calor que fluyen hacia los intervalos de temperatura contiguos inferiores y las cargas térmicas de servicios auxiliares requeridos.

No obstante, los problemas de PL-EM para determinar el número mínimo de apareamientos representan el principal cuello de botella en las estrategias secuenciales para la síntesis de redes de intercambio de calor. Furman y Sahinidis (2001) realizaron un análisis formal de complejidad computacional que revela que los modelos de transporte (Cerdá y Westerberg, 1983) y transbordo (Papoulias y Grossmann, 1983) se pueden catalogar como NP-difíciles en el sentido fuerte. Esto significa que a cualquier algoritmo de optimización tomará un tiempo no polinomial generar en una solución óptima, debido que las posibles combinaciones de apareamientos entre corrientes de proceso generan un crecimiento exponencial en el espacio de solución, produciendo la explosión combinatoria en problemas de tamaño considerable (30 corrientes de proceso).

Por otro lado, a pesar de las dificultades numéricas que los modelos de PL-EM pudieran exhibir, las metodologías secuenciales se consideran las más prácticas para abordar problemas de escala industrial debido a la oportunidad que ofrecen de descomponer el problema de síntesis (Chen y col. 2015). Por esta razón diferentes enfoques y algoritmos se han propuesto para enfrentar las limitaciones computacionales que surgen de este tipo de formulaciones. Furman y Sahinidis (2004) desarrollaron algoritmos de aproximación para el problema de número mínimo de apareamientos realizando un estudio para el modelo de transporte basado en técnicas de 
redondeo primario, redondeo doble, relajación lagrangiana, aproximación primaria-doble, y aproximación codiciosa.

Recientemente Chen y col. (2015) proponen estrategias computacionales recurriendo a técnicas de reformulación justas con relajaciones de PL e investigan diferentes aproximaciones para la solución del modelo de transbordo que incluyen desagregación del modelo y adición de cortes enteros para reducir los tiempos de solución. Las aproximaciones mencionadas permiten resolver problemas con tamaño considerable (30-40 corrientes) en tiempos computacionales razonables, sin embargo, al no ser algoritmos rigurosos las soluciones que se obtienen pudieran generar sobre estimaciones con respecto al número mínimo de equipos e incertidumbres en la selección de los apareamientos que se pudieran traducir en redes de intercambio de calor de costo poco atractivo.

\subsection{Alternativa al problema de número mínimo de apareamientos}

Incluso con el continuo desarrollo en resolvedores comerciales que permiten enfrentar problemas con un número de variables considerable, encontrar soluciones para el problema del número mínimo de apareamientos resulta una tarea compleja que no necesariamente concluye en configuraciones de redes con un costo competitivo. Esto pudiera motivar a desarrollar procedimientos para la síntesis de redes de intercambio de calor que posean las virtudes de las metodologías secuenciales para abordar el problema de síntesis de gran escala y que no tengan que lidiar con las limitaciones computacionales de las formulaciones de los modelos de PL-EM.

Debido a las ventajas que presentan los modelos de PL sobre los de PL-EM, particularmente en el problema de síntesis de redes de intercambio de calor, en este trabajo se plantea utilizar la solución del problema de uso mínimo de servicios auxiliares como medio para generar apareamientos de entre corrientes de proceso. El modelo de transporte otorga un conjunto de cargas térmicas que indican el calor intercambiado entre las corrientes de proceso las cuales pueden ser interpretadas como apareamientos termodinámicamente factibles. A su vez cada apareamiento puede ser igualmente asociado a un intercambiador de calor permitiendo construir diseños de red recurriendo únicamente a la formulación de PL. Se propone utilizar el modelo de 
transporte sobre el modelo de transbordo debido a que es posible tomar ventaja de la información que se guarda sobre los intervalos de temperatura de suministro y demanda.

Es posible anticipar que el problema de uso mínimo de servicios auxiliares genere una cantidad importante de apareamientos entre corrientes de proceso al no presentar un criterio que limite el intercambio de calor entre corrientes de proceso, no obstante, las estructuras de red diseñadas bajo estos conceptos imponen una cota superior en el número de equipos y definen una región factible acotada, evitando el crecimiento exponencial del problema al incrementar el número de corrientes. Por esto, resulta factible acoplar estas redes de intercambio de calor sobreestimadas a un modelo de programación matemática que realice una adecuada toma de decisiones para determinar, de entre los equipos disponibles, cuáles se deben conservar en el diseño final. 


\title{
Capítulo 3. Optimización de redes de intercambio de calor a partir de un diseño base
}

\author{
Notación \\ Índices \\ $k, k 1 \quad$ Índice para etapas y fronteras de etapas \\ $s, s 1 \quad$ Índice para corrientes \\ $h \quad$ Índice para corrientes de proceso calientes \\ c Índice para corrientes de proceso frías \\ $i \quad$ Iteración del algoritmo de multi-arranque \\ $L \quad$ Índice para la cota inferior de una variable de decisión \\ $U \quad$ Índice para la cota superior de una variable de decisión
}

\section{Conjuntos}

$S T$

K

$S$

$S P$

$S P O$

$S P 1$

$S S$

$S U$

$S_{k}$

$D_{s}$

$S 1_{k}$ $\{k: k$ es una etapa en el diagrama de malla $\}$

$\{k: k$ es una frontera de etapa en el diagrama de malla $\}$

$\{s: s$ es una corriente de proceso, sub-corriente o servicio auxiliar\}

$\{s: s$ es una corriente de proceso $\}$

$\{s: s$ es una corriente de proceso sin división de corrientes $\}$

$\{s: s$ es una corriente de proceso que se divide en sub-corrientes $\}$

$\{s: s$ es una sub-corriente $\}$

$\{s: s$ es una corriente de servicio auxiliar $\}$

$\{s \in S: s$ intercambia calor en la corriente $k\}$

$\{s 1 \in S S: s 1$ es una sub-corriente de la corriente de proceso $s\}$

$\{s \in S P 1$ : es una sub-corriente de $s$ que intercambia calor en la etapa $k\}$ 
$S 2_{k} \quad\{s \in S S$ : sub-corriente $s$ que no está presente en la etapa $k\}$

\section{Parámetros}

$\mathrm{F}, \mathrm{F}_{s}, \mathrm{~F}_{h}, \mathrm{~F}_{c} \quad$ Flujo de capacidad calorífica $\left(\mathrm{kW} \mathrm{K}^{-1}\right)$

TS, $\mathrm{TS}_{s} \quad$ Temperatura de suministro (K)

$\mathrm{TO}, \mathrm{TO}_{s} \quad$ Temperatura objetivo (K)

$\mathrm{C}_{s} \quad 1$ si es una corriente caliente

$\mathrm{C}_{s} \quad-1$ si es una corriente fría

$\mathrm{TOC}_{s} \quad 1$ si la temperatura objetivo está especificada

$\mathrm{TOC}_{s} \quad-1$ si la temperatura objetivo no está especificada

$\mathrm{h}, \mathrm{h}_{s} \quad$ Coeficiente de película para la transferencia de calor $\left(\mathrm{kW} \mathrm{m}^{-2} \mathrm{~K}^{-1}\right)$

$\mathrm{U}, \mathrm{U}_{k} \quad$ Coeficiente global de transferencia de calor $\left(\mathrm{kW} \mathrm{m}^{-2} \mathrm{~K}^{-1}\right)$

EMAT Diferencia mínima de temperaturas en intercambiador de calor

NOK Número total de etapas o intercambiadores de calor

$\mathrm{UC}_{k} \quad$ Costo unitario por servicio de calentamiento o enfriamiento $\left(\$ \mathrm{~kW}^{-1} \mathrm{yr}^{-1}\right)$

$\mathrm{CF}_{k} \quad$ Cargo fijo de costo de capital (\$)

$\mathrm{CC}_{k} \quad$ Coeficiente de ley de costo de capital $\left(\$ \mathrm{~m}^{-2 \beta_{k}}\right)$

$\beta_{k} \quad$ Exponente de ley de costo de capital

$\mathrm{r} \quad$ Factor de anualización para costo de capital $\left(\right.$ año $\left.^{-1}\right)$

$\mathrm{R}_{x}^{i} \quad$ Número aleatorio

$\mathrm{N} \quad$ Número total de iteraciones

CTA* Costo total anual de la mejor solución disponible $\left(\$\right.$ año $\left.{ }^{-1}\right)$

$\mathrm{CTA}_{i} \quad$ Costo total anual del diseño de red obtenido en la iteración $i\left(\$\right.$ año $\left.^{-1}\right)$

$\mathrm{Q}_{h} \quad$ Calor disponible $(\mathrm{kW}), \mathrm{Q}_{c}=\mathrm{F}_{c}\left(\mathrm{TO}_{c}-\mathrm{TS}_{c}\right)$

$\mathrm{Q}_{c} \quad$ Calor requerido $(\mathrm{kW}), \mathrm{Q}_{c}=\mathrm{F}_{c}\left(\mathrm{TO}_{c}-\mathrm{TS}_{c}\right)$ 


\begin{tabular}{|c|c|}
\hline $\mathrm{Q}_{\max }$ & $\begin{array}{l}\text { Intercambio de calor termodinámico máximo posible } \\
\mathrm{Q}_{\max }=\operatorname{Min}\left[\mathrm{F}_{h}, \mathrm{~F}_{c}\right]\left(\mathrm{TS}_{h}-\mathrm{TS}_{c}-\mathrm{EMAT}\right)\end{array}$ \\
\hline $\mathrm{TS}_{c}^{\min }$ & $\begin{array}{l}\text { Temperatura de suministro más baja de las corrientes frías con las } \\
\text { interactúa una sub-corriente caliente }(\mathrm{K})\end{array}$ \\
\hline $\mathrm{TS}_{h}^{\max }$ & $\begin{array}{l}\text { Temperatura de suministro más alta de las corrientes frías con las c } \\
\text { interactúa una sub-corriente fría }(\mathrm{K})\end{array}$ \\
\hline \multicolumn{2}{|c|}{ Variables continuas positivas } \\
\hline$q_{k}$ & Carga térmica de calor intercambiado en la etapa $k(\mathrm{~kW})$ \\
\hline$t_{s, k}$ & Temperatura de una corriente $s$ en la frontera caliente de la etapa $k(\mathrm{~K})$ \\
\hline$t_{s, k+1}$ & Temperatura de una corriente $s$ en la frontera fría de la etapa $k(\mathrm{~K})$ \\
\hline$d t h_{k}$ & Diferencia de temperaturas del lado caliente del intercambiador de calor $(\mathrm{K})$ \\
\hline$d t c_{k}$ & Diferencia de temperaturas del lado frío del intercambiador de calor (K) \\
\hline$\Delta T_{k}$ & Diferencia media logarítmica de temperaturas $(\mathrm{K})$ \\
\hline$\alpha_{s 1}$ & Fracción de flujo para la sub-corriente $s 1$ \\
\hline$A_{k}$ & Área de intercambio de calor $\left(\mathrm{m}^{2}\right)$ \\
\hline $\mathrm{CCT}_{k}$ & Costo de capital de intercambiador de calor $(\$)$ \\
\hline$z$ & Costo total anual de la función objetivo del modelo (P) de PNL \\
\hline$v$ & Variable de decisión del modelo (P) de PNL \\
\hline$x$ & Vector de variables de decisión del modelo $(\mathrm{P})$ \\
\hline$x^{i}$ & Solución óptima local del modelo (P) \\
\hline$x^{*}$ & Mejor solución óptima local del modelo (P) \\
\hline
\end{tabular}

\subsection{Metodologías para la optimización de redes de intercambio de calor}

En capítulos anteriores se han discutido de forma breve las dificultades que el sub-problema del número mínimo de apareamientos conlleva debido a las complejidades computacionales que los 
modelos de PL-EM presentan. Otra limitante de los enfoques secuenciales es la restricción en el tamaño de la red final impuesta al fijar en el mínimo al número apareamientos entre corrientes de proceso. Debido a las no convexidades involucradas en el problema de síntesis, una red con el número mínimo de equipos no necesariamente implica un diseño con CTA competitivo.

Los problemas mencionados pueden ser enfrentados si la selección de equipos es llevada a cabo sobre un conjunto definido de intercambios de calor que establezcan un diseño base y, mediante un esquema de optimización sea posible seleccionar aquellos que se traduzcan en la configuración que represente la mejor opción en términos económicos; teniendo como resultado una selección óptima de entre los equipos disponibles, a la par que se determinan los mejores valores posibles de las variables de decisión, evitando así la difícil tarea de resolver el problema de número mínimo de apareamientos.

Algunas de las metodologías de síntesis permiten generar diseños de redes de intercambio de calor con un número sobreestimado de equipos (e.g. Linnhoff y Hindmarsh, 1983; Briones y Kokossis, 1999; Shethna y col., 2000), estas redes poseen la característica de tener un dominio factible establecido y de presentar cierto número de grados de libertad. Diversos autores han desarrollado estrategias de optimización local, optimización global determinista y técnicas metaheurísticas, para minimizar el CTA de un diseño de red de intercambio de calor con topología preestablecida.

Cabe mencionar que aún para problemas donde el espacio de búsqueda está acotado por un diseño de red preestablecido, asegurar optimalidad global no resulta una tarea trivial. Westerberg y Shah (1978) realizaron avances iniciales en el desarrollo algoritmos de optimización para determinar el óptimo global para el diseño de red de intercambio de calor con estructura dada. Estos autores utilizan una estrategia de PNL basada en la descomposición Lagrangiana del problema en sub-sistemas, para posteriormente buscar el óptimo global de cada sistema.

Yee y Grossmann (1990) también abordan la optimización de estructuras fijas obtenidas a partir del modelo Synheat. Una vez definida una estructura óptima mediante PNL-EM, los autores plantean un problema de PNL para determinar los valores óptimos de las fracciones de flujos y temperaturas intermedias. Posteriormente Quesada y Grossmann (1993) proponen un algoritmo de optimización global determinista para estructuras fijas correspondientes a configuraciones particulares de la superestructura Synheat. Los autores utilizan un modelo de PNL que asume 
una función de costos como una función lineal del área y utilizan la DMAT para aproximar la DMLT, acoplado a una búsqueda de ramificación y acotamiento para establecer un óptimo global.

Ryoo y Sahinidis (1995) por otro lado desarrollaron un algoritmo para determinar el óptimo global en problemas de PNL y PNL-EM resolviendo una secuencia de problemas subestimadores que consisten de una relajación convexa, incrementando la región factible y subestimando la función objetivo, lo que resulta en una cota inferior para el mínimo global; también se construye una cota superior mediante técnicas de optimización local usando la solución relajada como punto inicial y posteriormente dividen la región factible repetidamente hasta que las subdivisiones posean cotas inferiores suficientemente cercanas a la mejor solución factible encontrada. Esto conduce a un método de búsqueda sobre un árbol de nodos, para lo que proponen un método de ramificación y reducción para la optimización global determinista. Los autores aplican el algoritmo para optimizar una red de intercambio de calor particular diseñada mediante la hiperestructura de Floudas y Ciric (1989).

Zamora y Grossmann (1998a) utilizan un algoritmo híbrido de ramificación y contracción para la optimización global determinista del modelo Synheat para la síntesis de redes de intercambio de calor. Los autores proponen un modelo de PNL-EM con funciones de costos lineal, sin división de corrientes y utilizan la DMAT como una aproximación suficiente de la DMLT. Posteriormente Zamora y Grossmann (1998b) proponen un algoritmo de ramificación y contracción resolviendo una secuencia de sub-problemas convexos que consisten en la contracción de cotas para el subconjunto de variables que participan en términos no convexos, para la optimización global continua de sistemas de procesos estructurados como es el caso de la optimización global determinista redes de intercambio de calor con estructura preestablecida resultantes de la metodología Synheat. Zamora y Grossmann (1997) usaron el algoritmo de ramificación y contracción para la síntesis de redes de intercambio de calor usando una familia de hiper-planos para la DMLT, con funciones de costos no lineales, pero manteniendo la restricción de diseño sin división de corrientes.

La principal desventaja de las técnicas de optimización determinista recae en que frecuentemente se encuentran limitadas a ciertas suposiciones o simplificaciones que permitan establecer una solución óptima global en tiempos de cómputo razonables. Por otro lado, las metodologías de 
búsqueda estocástica, aunque pudieran presentar incertidumbre en la obtención formal del óptimo global, han probado tener la capacidad de lidiar con diversos problemas de optimización sin verse restringidas por las no convexidades involucradas en los modelos.

Bochenek y col. (2007) utilizaron algoritmos genéticos para optimizar los valores de variables de decisión como fracciones de flujo y cargas térmicas de redes de intercambio de calor con topología fija, permitiendo eliminar equipos y divisiones de corrientes para reducir el CTA de la red. La metodología genera la población de descendientes a partir de un superconjunto de población de padres y de la subpoblación, en lugar de únicamente a partir de la población de padres como en la mayoría de los métodos que usan algoritmos genéticos, lo que reduce el efecto de degeneración prematura, previniendo la convergencia en un óptimo local.

Myankooh y Shafiei (2015) proponen la optimización de redes de intercambio de calor con configuración preestablecida mediante un algoritmo de colonia de hormigas, permitiendo reexaminar las redes de intercambio de calor previamente diseñadas y obtener soluciones óptimas más económicas, pero limitándose a evaluar estructuras sin división de corrientes. La técnica consiste en generar soluciones factibles iniciales aleatorias usando una distribución probabilística, conservando las mejores soluciones candidatas para modificar la distribución. Esto mejora la posibilidad de obtener soluciones de mejor calidad a través de un número de iteraciones.

Recientemente Núñez-Serna y Zamora (2016) proponen una alternativa que consiste en un algoritmo de optimización local con multi-arranque estocástico para redes de intercambio de calor con estructura preestablecida. Esta técnica iterativa combina la inicialización pseudoaleatoria para los valores de las variables de decisión con un modelo de PNL para la optimización. La metodología posee la característica reducir la probabilidad de caer en un único óptimo local de baja calidad al explorar eficientemente diversas zonas de la región factible mediante la propuesta de múltiples puntos de arranque, permitiendo exponer una colección de soluciones óptimas locales. La estrategia propuesta por Núñez-Serna y Zamora (2016) permite optimizar una variedad de posibles configuraciones que surgen típicamente de la mayoría de las metodologías de síntesis, con características que incluyen divisiones de corrientes y mezclado no isotérmico, pero a lo más una división en cada corriente. Los autores optimizan eficientemente 
dos estructuras con topología preestablecida que corresponden a problemas de mediana escala, disminuyendo el CTA en comparación con los diseños iniciales.

El enfoque propuesto por Núñez-Serna y Zamora (2016) representa una herramienta versátil que puede ser acoplada como paso final en algunas metodologías de síntesis para evaluar el diseño de red obtenido, llevando al límite posibles mejoras en el CTA redistribuyendo los valores de las variables de decisión. Por otro lado, debido a las características sistemáticas propias de la metodología, pudiera resultar adecuada para su implementación como un sub-problema de síntesis de redes de intercambio de calor donde no se considera el mínimo número de apareamientos, en cambio se busca optimizar un diseño base que requiere una apropiada toma de decisiones para llevar a cabo reducciones estructurales que conduzcan a redes con CTA óptimo.

\subsection{Algoritmo de optimización multi-arranque estocástico en dos fases}

Para la formulación de la metodología de multi-arranque estocástico en dos fases (Núñez-Serna y Zamora, 2016), se deben declarar los elementos que conforman una red con estructura preestablecida sujeta a optimización haciendo uso de conceptos fundamentales de la teoría de conjuntos y de una representación por etapas que se puede visualizar como un diagrama de malla (Flower y Linnhoff, 1983). La Figura 3.1 muestra un ejemplo ilustrativo de una red de intercambio de calor con topología preestablecida donde cada intercambiador de calor entre corrientes de proceso y servicios auxiliares se define como una etapa independiente $k$ en el diagrama de malla.

La estructura descrita en la Figura 3.1 presenta 7 equipos cada uno ubicado dentro de una etapa y delimitados por sus respectivas fronteras; en general si están presentes NOK etapas, entonces existen $\mathrm{NOK}+1$ fronteras. Los equipos etiquetados 2, 3, 4 y 5 son recuperadores de calor, mientras que el equipo 1 representa los servicios de calentamiento y los equipos 6 y 7 servicios de enfriamiento. Cada corriente de proceso, sub-corriente y corriente de servicio auxiliar recibe una numeración única. Las corrientes 2 y 5 representan corrientes de proceso calientes, las corrientes 3 y 4 son sub-corrientes de la corriente H1, las corrientes 6 y 9 describen corrientes de 
proceso frías, donde 7 y 8 son sub-corrientes de la corriente C1. Los servicios de calentamiento se ubican en la corriente 1 y los servicios de enfriamiento en las corrientes 10 y 11 .

En la Figura 3.1 también es posible visualizar las posibles configuraciones que la metodología admite, como son arreglos secuenciales de equipos en corrientes de proceso (etapas 2 y 3); arreglos secuenciales ubicados en sub-corrientes de proceso (etapas 4 y 6), equipos en paralelo (etapas 4 y 5), servicios auxiliares en corrientes de proceso (etapa 1 y 7 ) y servicios auxiliares en sub-corrientes de proceso (etapa 6).

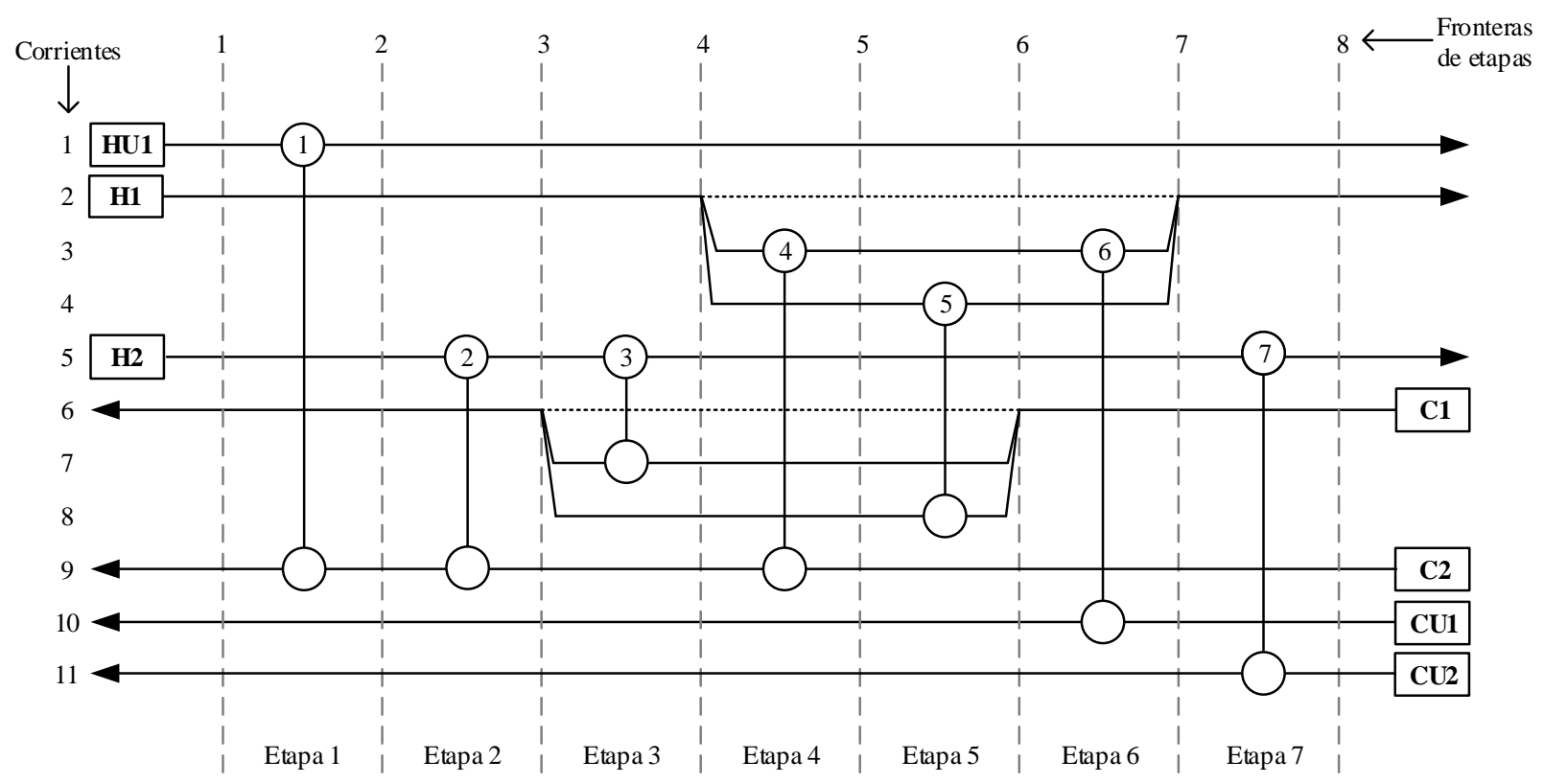

Figura 3.1. Representación de una red de intercambio de calor con estructura fija.

La teoría de conjuntos provee de las herramientas necesarias para que una red de intercambio de calor con configuración preestablecida se pueda interpretar como la entrada de un modelo de programación matemática. Los conjuntos por definir se especifican como. Conjunto de etapas del diagrama de malla $S T=\{1,2,3,4,5,6\}$, conjunto de fronteras de etapas $K=\{1,2,3,4,5,6,7,8\}$, conjunto de corrientes $S=\{1,2,3,4,5,6,7,8,9,10,11\}$, conjunto de corrientes de proceso $S P=\{2,5,6,9\}$, conjunto de corrientes sin división $S P 0=\{5,9\}$, conjunto de corrientes con división $S P 1=\{2,6\}$, conjunto de sub-corrientes $S S=\{3,4,7,8\}$, conjunto de servicios auxiliares $S U=\{1,10,11\}$, conjuntos que definen los intercambios de calor $S_{1}=\{1,9\}$, $S_{2}=\{5,9\}, \quad S_{3}=\{5,7\}, \quad S_{4}=\{3,9\}, \quad S_{5}=\{4,8\}, \quad S_{6}=\{3,10\}, \quad S_{7}=\{5,11\}$, conjuntos que 
establecen la relación entre una corriente de proceso y sus sub-corrientes $D_{2}=\{3,4\}$ y $D_{6}=\{7,8\}$, conjuntos que identifican a las corrientes de proceso teniendo una sub-corriente intercambiando calor en una etapa dada $S 1_{3}=\{7\}, S 1_{4}=\{3\}, S 1_{5}=\{4\}, S 1_{5}=\{8\}, S 1_{6}=\{3\}$, y conjuntos que especifican las sub-corrientes que no están presentes en una etapa $S 2_{1}=\{3,4,7,8\}$ , $S 2_{2}=\{3,4,7,8\}, S 2_{3}=\{3,4\}, S 2_{6}=\{7,8\}, S 2_{7}=\{3,4,7,8\}$.

El modelo de PNL desarrollado por Núñez-Serna y Zamora (2016) para la minimización del CTA de una red con topología preestablecida haciendo uso de los conjuntos establecidos se describe a continuación.

\section{Modelo (P)}

Función objetivo

Minimizar $\quad z=\sum_{k \in S T}\left[\mathrm{CF}_{k} \cdot \mathrm{r}+\mathrm{CC}_{k} \cdot \mathrm{r} \cdot\left(\frac{q_{k}}{\mathrm{U}_{k} \Delta T_{k}}\right)^{\beta_{k}}+\mathrm{UC}_{k} \cdot q_{k}\right]$

donde $0<\beta_{k} \leq 1 \quad$ y $\quad \mathrm{U}_{k}=\left[\sum_{s \in S_{k}}\left(\frac{1}{\mathrm{~h}_{s}}\right)\right]^{-1}, k \in S T$;

\section{Restricciones del modelo}

Balances globales de calor por corriente

$$
\sum_{\substack{k \in S T: \\ s \in S_{k} \cup S 1_{k}}} q_{k}=\mathrm{F}_{S} \cdot \mathrm{C}_{s} \cdot\left(\mathrm{TS}_{s}-\mathrm{TO}_{s}\right) \quad s \in S P \text { con } \mathrm{TOC}_{s}=1
$$

Balances de calor en cada intercambiador

$$
\begin{array}{ll}
q_{k}=\mathrm{F}_{s}\left(t_{s, k}-t_{s, k+1}\right) & s \in S P \cap S_{k}, k \in S T \\
q_{k}=\mathrm{F}_{s} \cdot \alpha_{s 1}\left(t_{s 1, k}-t_{s 1, k+1}\right) & s: s 1 \in D_{s}, s 1 \in S S \cap S_{k}, k \in S T
\end{array}
$$

Balances de masa en divisores de corriente 
$\sum_{s 1 \in D_{s}} \alpha_{s 1}=1 \quad s \in S P 1$

Diferencias de temperaturas

$$
\begin{aligned}
& d t h_{k}=\sum_{s \in S_{k}} \mathrm{C}_{s} \cdot t_{s, k} \quad k \in S T \\
& d t c_{k}=\sum_{s \in S_{k}} \mathrm{C}_{s} \cdot t_{s, k+1} \quad k \in S T
\end{aligned}
$$

Diferencia media logarítmica de temperaturas

$$
\Delta T_{k}=\frac{\left(d t h_{k}-d t c_{k}\right)}{\ln \left(d t h_{k} / d t c_{k}\right)} \quad k \in S T
$$

Propagación horizontal de temperaturas

$$
\begin{aligned}
& t_{s, k}=t_{s, k+1} \quad s \in S P 0 \backslash S_{k}, \quad k \in S T \\
& t_{s, k}=t_{s, k+1} \quad s \in S P 1 \backslash\left(S_{k} \cup S 1_{k}\right), \quad k \in S T \\
& t_{s, k}=t_{s, k+1} \quad s \in S S \backslash\left(S_{k} \cup S 2_{k}\right), k \in S T \\
& t_{s, k}=t_{s, k+1} \quad s \in S U \backslash S_{k}, \quad k \in S T \\
& q_{k}=\mathrm{F}_{s}\left(t_{s, k}-t_{s, k+1}\right) \quad s \in S 1_{k}, \quad k \in S T
\end{aligned}
$$

Propagación vertical de temperaturas

$$
\begin{array}{lll}
t_{s 1,1}=t_{s, 1} \quad s 1 \in D_{s}, & s \in S P 1 \text { con } \mathrm{C}_{s}=1 \\
t_{s 1, k+1}=t_{s, k+1} \quad s 1 \in D_{s} \cap S 2_{k}, & s \in S P 1 \operatorname{con} \mathrm{C}_{s}=1, \quad k \in S T \\
t_{s 1, N O K+1}=t_{s, N O K+1} \quad s 1 \in D_{s}, & s \in S P 1 \operatorname{con} \mathrm{C}_{s}=-1 \\
t_{s 1, k}=t_{s, k} & s 1 \in D_{S} \cap S 2_{k}, & s \in S P 1 \text { con } \mathrm{C}_{s}=-1, k \in S T
\end{array}
$$

Asignación de temperaturas de suministro y objetivo

$$
\begin{array}{ll}
t_{s, 1}=\mathrm{TS}_{s} & s \in S P \cup S U \text { con } \mathrm{C}_{s}=1 \\
t_{s, N O K+1}=\mathrm{TO}_{s} & s \in S P \cup S U \text { con } \mathrm{C}_{s}=1 \text { y } \mathrm{TOC}_{s}=1
\end{array}
$$




$$
\begin{array}{ll}
t_{s, N O K+1}=\mathrm{TS}_{s} & s \in S P \cup S U \text { con } \mathrm{C}_{s}=-1 \\
t_{s, 1}=\mathrm{TO}_{s} & s \in S P \cup S U \operatorname{con} \mathrm{C}_{s}=-1 \text { y } \mathrm{TOC}_{s}=1
\end{array}
$$

\section{Cotas}

$$
\begin{array}{ll}
q_{k}{ }^{L} \leq q_{k} \leq q_{k}^{U} & k \in S T \\
t_{s, k}{ }^{L} \leq t_{s, k} \leq t_{s, k} U & s \in S, k \in S T \\
d t h_{k}^{L} \leq d t h_{k} \leq d t h_{k}^{U} & k \in S T \\
d t c_{k}^{L} \leq d t c_{k} \leq d t c_{k}^{U} & k \in S T \\
\Delta T_{k}{ }^{L} \leq \Delta T_{k} \leq \Delta T_{k}^{U} & k \in S T \\
0 \leq \alpha_{s 1} \leq 1 &
\end{array}
$$

La DMLT expresada en la ecuación (3.8) se indetermina en el caso donde $d t h_{k}=d t c_{k}$, para evitar dichos inconvenientes Underwood (1970), Paterson (1984) y Chen (1987) proponen diferentes aproximaciones que no presentan dicho problema. En este trabajo se elige utilizar la aproximación de Paterson para sustituir la DMLT del modelo (P).

Aproximación de Paterson para la diferencia media logarítmicade temperaturas

$$
\Delta T_{k}=\frac{2}{3} \sqrt{d t h_{k} \cdot d t c_{k}}+\frac{1}{3}\left(\frac{d t h_{k}+d t c_{k}}{2}\right) \quad k \in S T
$$

\section{Estrategia de optimización}

Existe una fuerte dependencia entre la inicialización de las variables y la calidad de la solución debido a las características no convexas del modelo (P) ocasionadas por las no linealidades presentes en la definición de la DMLT y sus aproximaciones, en la función objetivo (función cóncava univariada), en el cálculo del área de intercambio de calor (términos fraccionales lineales) y en los balances de energía donde hay división de corrientes (términos bilineales). Es probable que una solución obtenida del modelo de PNL represente un óptimo local; por esta razón, es necesario considerar una variedad de puntos de arranque que permitan realizar una búsqueda eficiente sobre el espacio de solución para evaluar los posibles múltiples óptimos locales contenidos en el espacio factible del diseño base, esto incrementa la posibilidad de 
obtener una buena solución óptima local y en el mejor de los casos determinar una solución óptima global.

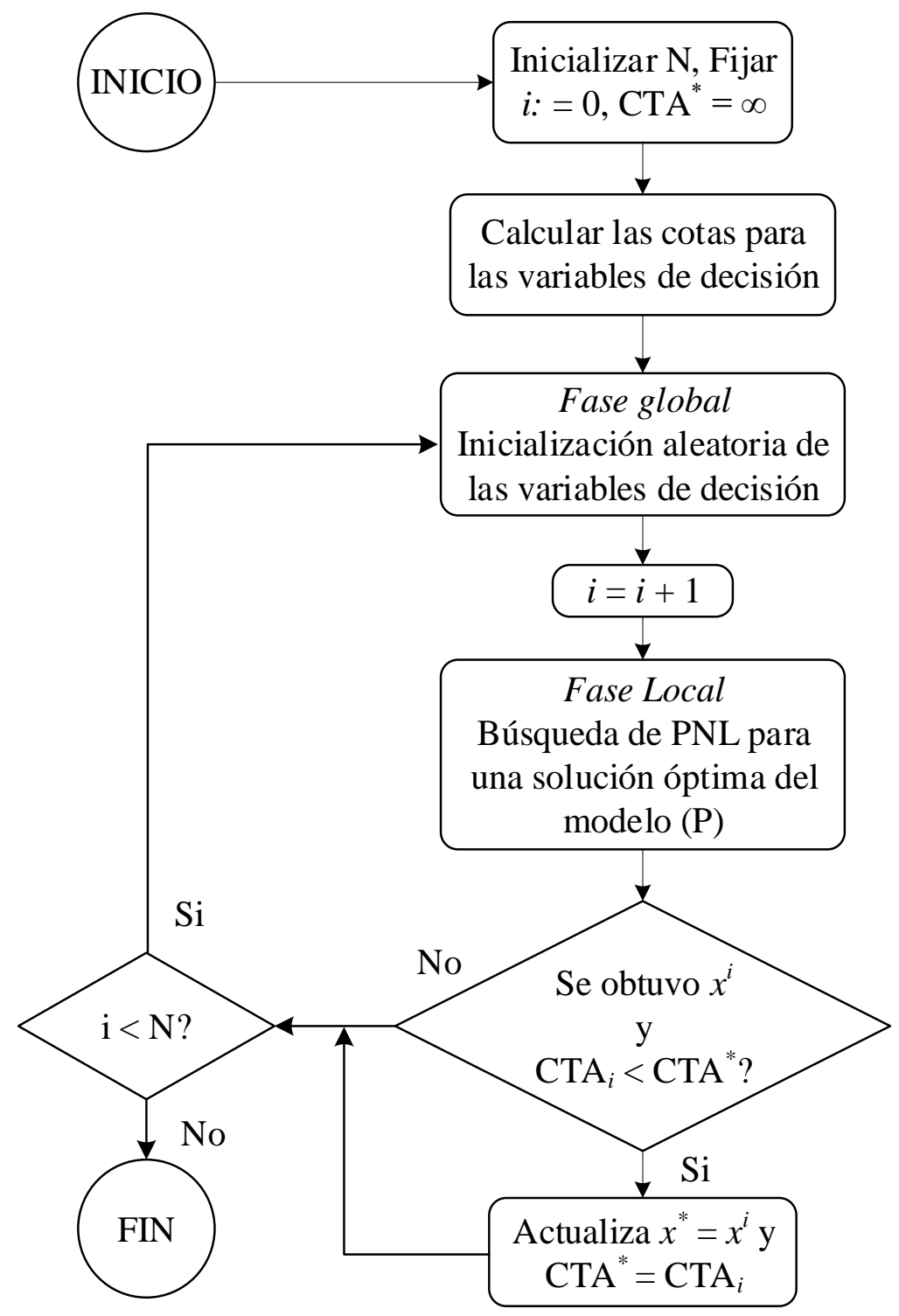

Figura 3.2. Algoritmo de optimización de multi-arranque estocástico en dos fases (Núñez-Serna y Zamora, 2016).

Núñez-Serna y Zamora (2016) proponen una estrategia de optimización de multi-arranque estocástico que consiste en dos fases que se realizan a lo largo de un número $\mathrm{N}$ de iteraciones establecidas por el usuario. Para cada iteración $i$, en la primera fase o fase global se definen valores de las variables de decisión como son cargas térmicas, temperaturas intermedias, diferencias de temperatura y fracciones de flujo, de forma pseudo-aleatoria tomando valores del 
dominio factible delimitado por cotas previamente calculadas. En la segunda fase o fase local se resuelve el modelo (P) de PNL en busca de un óptimo local $x^{i}$ considerando los valores de inicialización de la fase uno. El algoritmo se inicia definiendo el costo de la mejor solución disponible en $\mathrm{CTA}^{*}=\infty$, y se guarda registro si en alguna iteración se ha conseguido una solución de menor costo, actualizando tanto el vector que contiene a las variables de decisión de modo que $x^{*}=x^{i}$, como el costo de la mejor solución disponible en $C T A^{*}=C T A_{i}$. La detección y comparación de óptimos locales de menor costo incrementan las posibilidades de obtener soluciones de calidad.

La técnica de multi-arranque consiste de asignar valores pseudo-aleatorios independientes en

cada iteración, definidos entre los valores de la cota superior $v^{U}$ y la cota inferior $v^{L}$ para cada variable de decisión $v$ por medio de la siguiente expresión.

$v=v^{L}+R_{x}^{i}\left(v^{U}-v^{L}\right)$

Donde, para cada inicialización $R_{x}^{i}$ es un número aleatorio con distribución probabilística uniforme en el rango de valores entre [0,1]. En la Figura 3.2 se muestra el diagrama de flujo del algoritmo multi-arranque estocástico en dos fases.

\subsection{Estructuras redundantes como diseño base para la optimización}

Existe la posibilidad de disminuir el tamaño de un diseño base con estructura preestablecida mediante la metodología de multi-arranque estocástica en dos fases. Esta característica puede ser explotada al proponer redes factibles de intercambio de calor a optimizar, que posean una sobreestimación en cuanto número de intercambios de calor se refiere. Estas redes se dicen redundantes pues resulta evidente que no todos los equipos son necesarios para llevar a cabo una tarea de recuperación de calor eficiente, lo que se traduce en redes con costos probablemente poco atractivos. Sin embargo, la característica destacable de estas redes es que cuentan con cierto número de grados de libertad, lo que supone en un amplio espacio de búsqueda que se encuentra acotado por la topología preestablecida. 
El método de optimización de multi-arranque estocástico en dos fases realiza sistemáticamente en cada iteración la toma de decisiones mediante el modelo de PNL, lo que ofrece la posibilidad de eliminar equipos redundantes del diseño base cuando la carga térmica de esos equipos es llevada a cero al minimizar el CTA. Esto significa que, para una estructura de red redundante dada, la metodología de optimización busca realizar un balance adecuado entre el calor recuperado, área de intercambio de calor y el CTA de la red.

El criterio básico para considerar un diseño base como red de intercambio de calor redundante es que posea al menos un equipo que pueda ser eliminado por la estrategia de optimización, en comparación con el diseño de red inicial. Las redes redundantes se pueden obtener de algún esquema de síntesis que no tenga la capacidad de generar redes de intercambio de calor óptimas, lo que usualmente permite mejoras en el CTA del diseño base después de ser optimizadas.

Para ejemplificar los conceptos descritos en esta sección, se retoma el ejemplo ilustrativo 2.1 de la Sección 2.2 cuyos datos fueron extraídos de un problema propuesto por Zamora y Grossmann (1997). En la Tabla 3.1 se detallan los datos de corrientes y se incluyen las funciones de costos y los respectivos costos de servicios auxiliares que caracterizan al problema.

Tabla 3.1. Datos de corrientes y costos del ejemplo ilustrativo 3.1.

\begin{tabular}{|c|c|c|c|c|}
\hline Corriente & $\begin{array}{l}\text { Temperatura de } \\
\text { suministro } \\
\operatorname{Ts}\left({ }^{\circ} \mathrm{C}\right)\end{array}$ & $\begin{array}{l}\text { Temperatura } \\
\text { objetivo } \\
\mathrm{To}\left({ }^{\circ} \mathrm{C}\right)\end{array}$ & $\begin{array}{c}\text { Flujo de capacidad } \\
\text { calorífica } \\
\mathrm{F}\left(\mathrm{kW}^{\circ} \mathrm{C}^{-1}\right)\end{array}$ & $\begin{array}{l}\text { Coeficiente de } \\
\text { película } \\
\mathrm{h}\left(\mathrm{kW} \mathrm{m}^{-1}{ }^{\circ} \mathrm{C}^{-1}\right)\end{array}$ \\
\hline $\mathrm{H} 1$ & 180 & 75 & 30 & 0.15 \\
\hline $\mathrm{H} 2$ & 240 & 60 & 40 & 0.10 \\
\hline $\mathrm{C} 1$ & 40 & 230 & 35 & 0.20 \\
\hline $\mathrm{C} 2$ & 120 & 300 & 20 & 0.10 \\
\hline HU & 325 & 325 & - & 2 \\
\hline $\mathrm{CU}$ & 25 & 40 & - & 0.50 \\
\hline
\end{tabular}

Costo de intercambiadores de calor y enfriadores $\left(\$\right.$ año $\left.^{-1}\right)=1500+30\left[\text { Área }\left(\mathrm{m}^{2}\right)\right]^{0.8}$

Costo de calentadores $\left(\$\right.$ año $\left.^{-1}\right)=1500+60\left[\text { Área }\left(\mathrm{m}^{2}\right)\right]^{0.8}$

Costo de servicios de calentamiento $=110\left(\$ \mathrm{~kW}^{-1}\right.$ año $\left.^{-1}\right)$

Costo de servicios de enfriamiento $=10\left(\$ \mathrm{~kW}^{-1} \mathrm{año}^{-1}\right)$

Se considera que para el ejemplo ilustrativo 3.1 se cuenta con una estructura de red dada, mostrada en la Figura 3.3, en donde es posible asegurar que los correspondientes intercambios de 
calor entre corrientes de proceso son factibles, pero presentan un excedente con relación al número óptimo de equipos, por lo que se puede asumir que existe la posibilidad de que al menos un intercambiador de calor puede ser eliminado. El diagrama de malla representado en la Figura 3.3 es admitido por la metodología de multi-arranque estocástico en dos fases (Núñez-Serna y Zamora, 2016) como se muestra en la Sección 3.2, requiriendo únicamente los elementos estructurales del diseño como entrada del modelo. El algoritmo de optimización permite determinar los valores óptimos de las variables de decisión, para llevar a cabo reducciones estructurales que se traduzcan en soluciones simplificadas con CTA óptimo contenidas en la topología redundante.

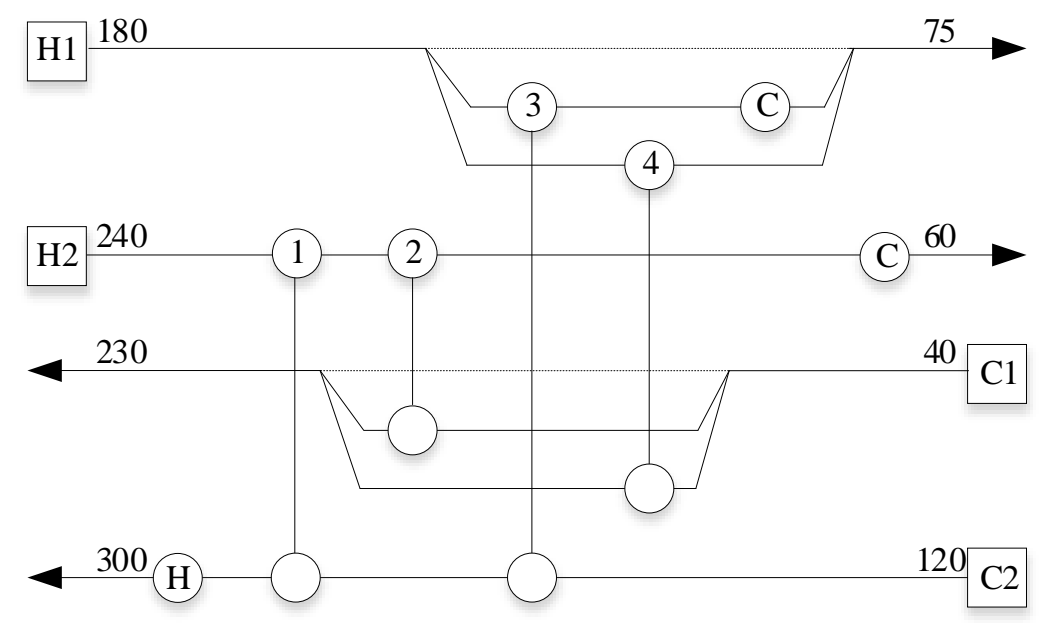

Figura 3.3. Red redundante 3-R1.

\subsection{Multiplicidad de óptimos locales}

Las no linealidades del modelo descrito en la Sección 3.2 dan lugar a no convexidades que, a su vez, conducen a la posibilidad de obtener múltiples óptimos locales. El método de solución ofrece la oportunidad de optimizar redes de intercambio de calor con topología preestablecida que pudieran contener una o varias soluciones óptimas locales que en principio no eran evidentes para el diseñador.

La aplicación de la estrategia de optimización para la red redundante 3-R1 propuesta en la Figura 3.3 se lleva a cabo en el ambiente GAMS 21.4 utilizando CONOPT v.3 como resolvedor de 
PNL. A pesar de que el problema posee un número modesto de intercambiadores de calor, después de 150 iteraciones es posible distinguir cuatro diferentes óptimos locales cuyas reincidencias se muestran en la Figura 3.4. Distintos equipos fueron eliminados en cada solución por el modelo de PNL exponiendo diseños de redes de intercambiadores de calor con diferente CTA, las estructuras de red resultantes de la optimización de la red redundante se presentan en la Figura 3.5.

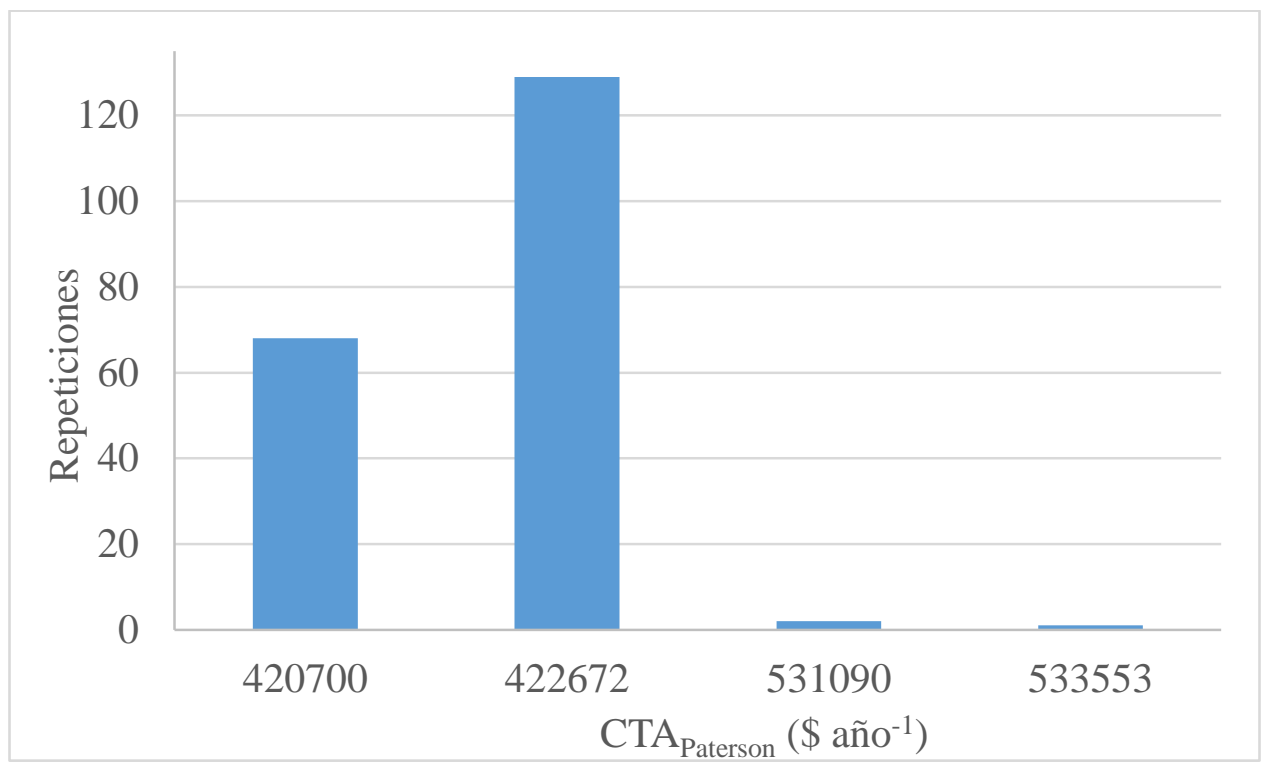

Figura 3.4. Histograma de soluciones óptimas locales obtenidas fijando 150 inicializaciones para el diseño base 3-R1.

En la Figura 3.5 se puede apreciar que para el diseño de red inicial 3-R1, ningún óptimo local requiere divisores de corrientes y se muestra que al eliminar una mayor cantidad de equipos se recupera menos calor por lo que es necesario hacer un mayor uso de servicios auxiliares incrementando en consecuencia el CTA de los diseños más reducidos. En a Tabla 3.2 se resumen los resultados de cada diseño de red simplificado obtenido. Se puede señalar que la mejor solución con un $\mathrm{CTA}_{\text {Paterson }}$ de $\$ 420,699.51$ mostrada en la Figura 3.5a, posee la mayor área de intercambio de calor en comparación con el resto de las soluciones, lo anterior sugiere que una menor área de intercambio de calor o un número reducido de equipos, no necesariamente involucra diseños finales de menor costo. Por lo que el método de optimización empleado resulta adecuado para la evaluación de diferentes soluciones óptimas locales donde cada una pudiera 
presentar diferentes características, permitiendo identificar las redes con un nivel adecuado de recuperación de calor y área de intercambio de calor permitiendo descartar los diseños finales con costos elevados.

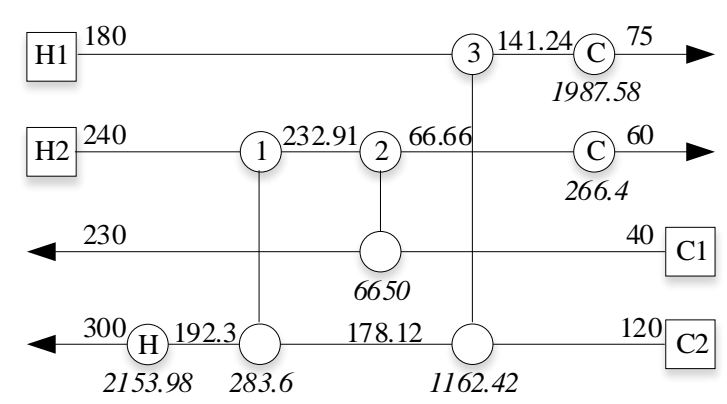

a) Óptimo local con CTA Paterson de $\$ 420,699.51$

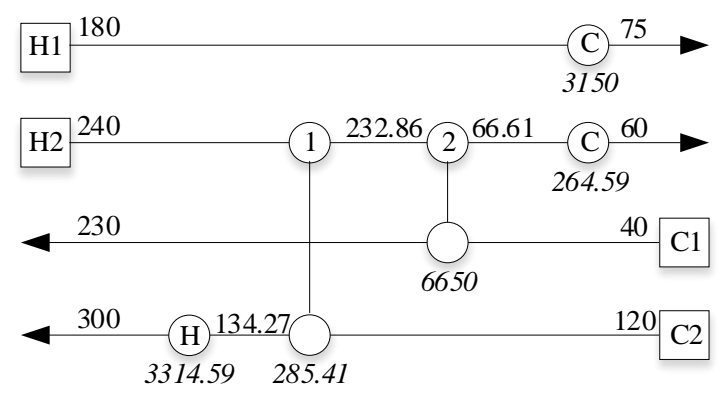

c) Óptimo local con CTAPaterson de

$\$ 531,089.72$

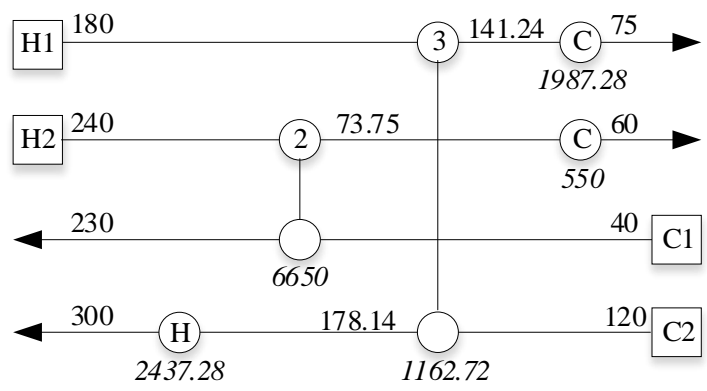

b) Óptimo local con CTAPaterson de $\$ 422,672.46$

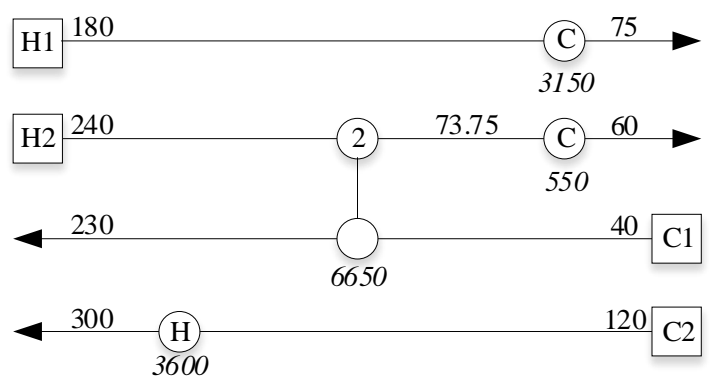

d) Óptimo local con CTA Paterson de $\$ 533,552.89$

Figura 3.5. Diferentes soluciones óptimas locales contenidas en la estructura 3-R1, para el ejemplo ilustrativo 3.1(temperaturas $\left({ }^{\circ} \mathrm{C}\right)$ en fuente normal, cargas térmicas $(\mathrm{kW})$ en cursivas y fracciones de flujo entre paréntesis).

El análisis de las diferentes soluciones óptimas locales resulta de gran utilidad pues deja en visto diferentes aspectos, no solo cuantitativos, medidos por el CTA de la red, sino también cualitativos como pudieran ser estructuras más simples que mejoren la operabilidad y controlabilidad de la planta, dando al diseñador la opción de elegir el diseño final que implique un mayor beneficio tomando, en cuenta más de un solo criterio.

Los aspectos de aleatoriedad que caracterizan a la metodología de multi-arranque estocástica en dos fases representan la posibilidad de evaluar diferentes zonas del espacio factible y de obtener diferentes soluciones óptimas locales, sin embargo, como se observa en la Figura 3.4 existen soluciones a las que no se converge con frecuencia. Los óptimos locales representados en las 
Figuras $3.5 \mathrm{c}$ y $3.5 \mathrm{~d}$ sólo se obtienen dos y una vez, respectivamente, lo que sugiere la posibilidad de excluir una o varias soluciones óptimas locales, independientemente de que posean un CTA competitivo o no. Esta característica teóricamente se podría contrarrestar si se realizaran un número infinito de iteraciones lo que implicaría un tiempo de cómputo infinito. Como se desea determinar soluciones en tiempos razonables, es imposible asegurar, con el método estocástico utilizado, la optimalidad global de una red redundante de intercambio de calor. No obstante, como se muestra en el ejemplo ilustrativo 3.1, es posible admitir que después de un número considerable de iteraciones para un cierto diseño base, pudiera resultar poco probable obtener nuevas soluciones con un menor CTA.

Tabla 3.2. Resumen de soluciones óptimas locales que contiene el diseño base 3-R1, fijando 150 inicializaciones.

\begin{tabular}{cccccc}
\hline $\begin{array}{c}\text { CTA } \\
\left(\$ \text { año }^{-1}\right)\end{array}$ & $\begin{array}{c}\text { Número } \\
\text { de } \\
\text { equipos }\end{array}$ & $\begin{array}{c}\text { Calor } \\
\text { recuperado } \\
(\mathrm{kW})\end{array}$ & $\begin{array}{c}\text { Servicios de } \\
\text { calentamiento } \\
(\mathrm{kW})\end{array}$ & $\begin{array}{c}\text { Servicios de } \\
\text { enfriamiento } \\
(\mathrm{kW})\end{array}$ & $\begin{array}{c}\text { Área } \\
\left(\mathrm{m}^{2}\right)\end{array}$ \\
\hline $420,699.51$ & 6 & 8,096 & 2,154 & 2,254 & 12,439 \\
$422,672.46$ & 5 & 7,813 & 2,437 & 2,537 & 83,13 \\
$531,089.72$ & 5 & 6,935 & 3,315 & 3,415 & 10,185 \\
$533,552.89$ & 4 & 6,650 & 3,600 & 3,700 & 6,049 \\
\hline
\end{tabular}

\subsection{Riesgos y beneficios de la optimización de redes redundantes}

Como se puede predecir hasta ahora, una limitante de la optimización de redes redundantes recae en que las soluciones finales solo pueden estar contenidas en la topología preestablecida, por esta razón un diseño base con un número considerable de intercambios de calor, en otras palabras, que posea un alto nivel de redundancia, pudiera incrementar la posibilidad de contener alguna solución con CTA competitivo. Un mayor número de grados de libertad implica un espacio de búsqueda más amplio y en principio, la posibilidad de revelar un mayor número de óptimos locales, en contraparte, esta propuesta conlleva a la necesidad de realizar una búsqueda más 
exhaustiva con el fin de disminuir las probabilidades de omitir un óptimo local con CTA competitivo.

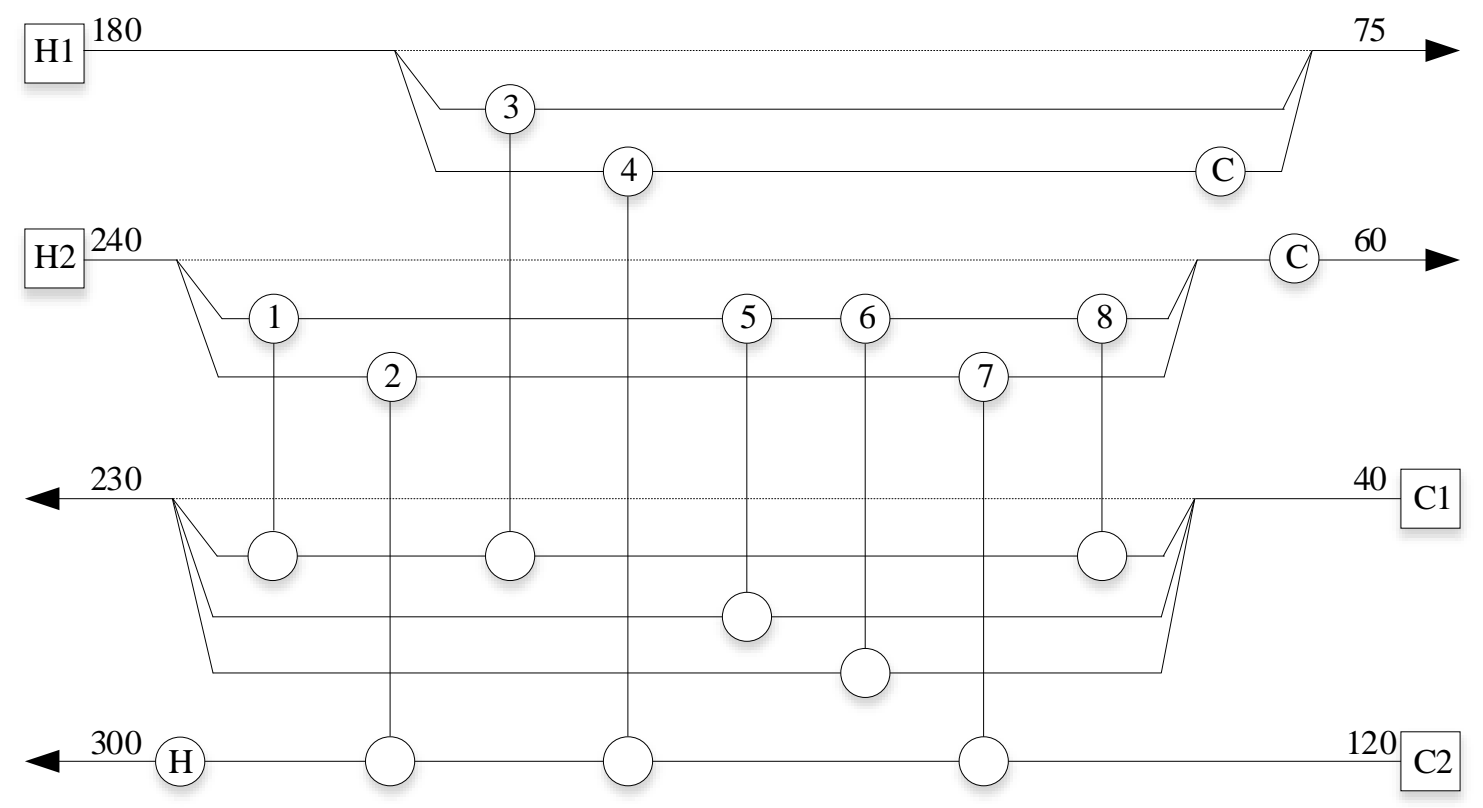

Figura 3.6. Red redundante 3-R2.

En las Figuras 3.6 y 3.7 se proponen dos nuevas estructuras redundantes para el ejemplo ilustrativo 3.1. Ambos diseños presentan once intercambiadores de calor y los mismos divisores de corrientes, pero exhiben diferencias en su configuración. Dichas redes redundantes fueron diseñadas con la premisa de contener la red redundante 3-R1 descrita en la Figura 3.3 bajo la hipótesis de que deben incluir, al menos, los mismos óptimos locales determinados en la Sección 3.4 .

El diseño base 3-R2 representado en la Figura 3.6 involucra numerosos re-apareamientos entre las corrientes $\mathrm{H} 2$ y $\mathrm{C} 1$, contando con cinco de los ocho recuperadores de calor totales de la estructura, lo que limita en cierta medida la oportunidad de explorar diversas opciones de intercambio de calor entre las corrientes de proceso restantes. En la Figura 3.8 se describen los resultados de la optimización de la estructura redundante 3-R2 fijando 250 inicializaciones aleatorias. Para este caso, se establecen doce óptimos locales diferentes donde la mejor solución presenta un $\mathrm{CTA}_{\text {Paterson }}$ de $\$ 422,169.76$, notando que, para el número de iteraciones realizadas no es posible detectar la mejor solución obtenida anteriormente en la estructura 3-R1 con CTA Paterson $_{\text {}}$ de $\$ 420,699.51$. 


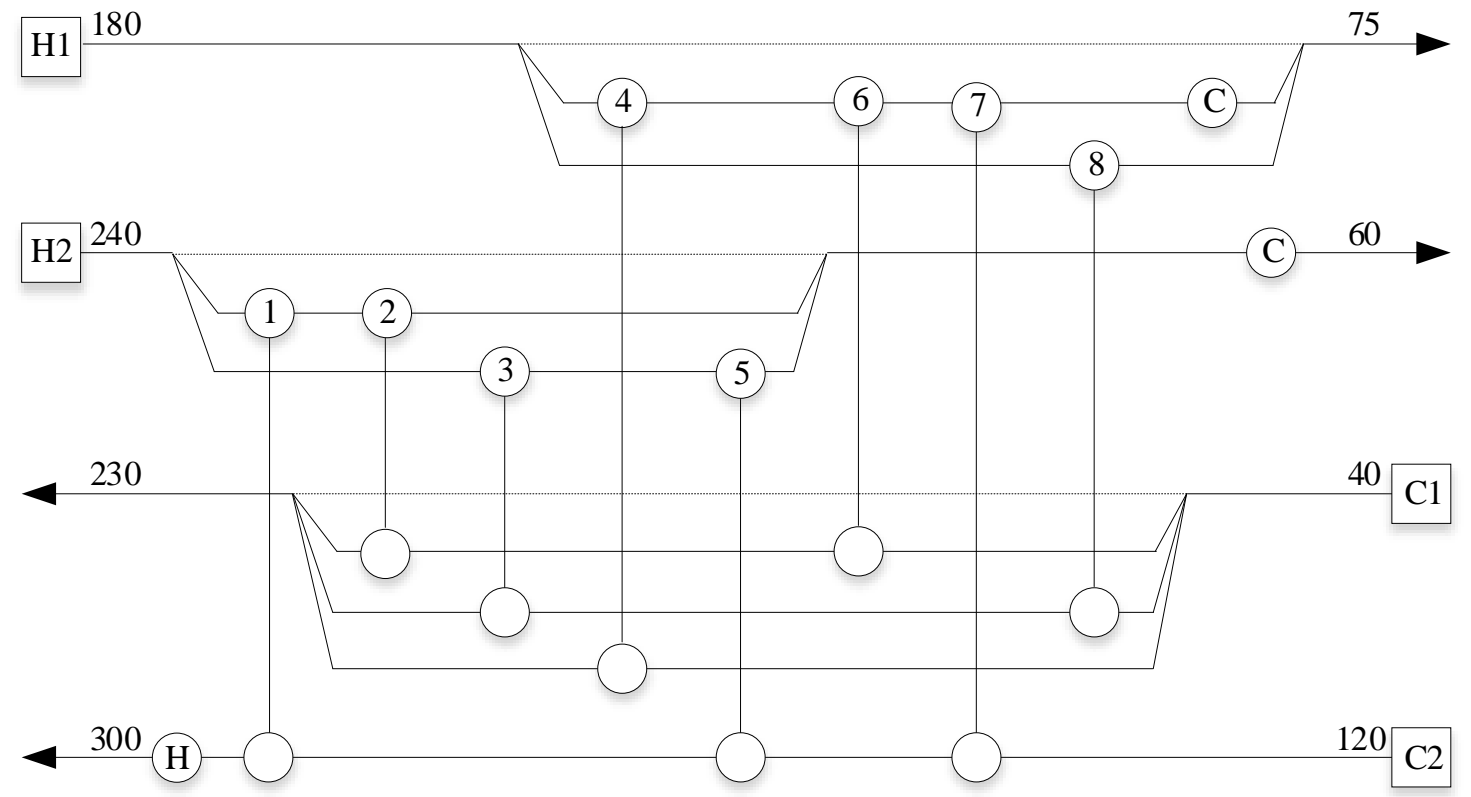

Figura 3.7. Red redundante 3-R3

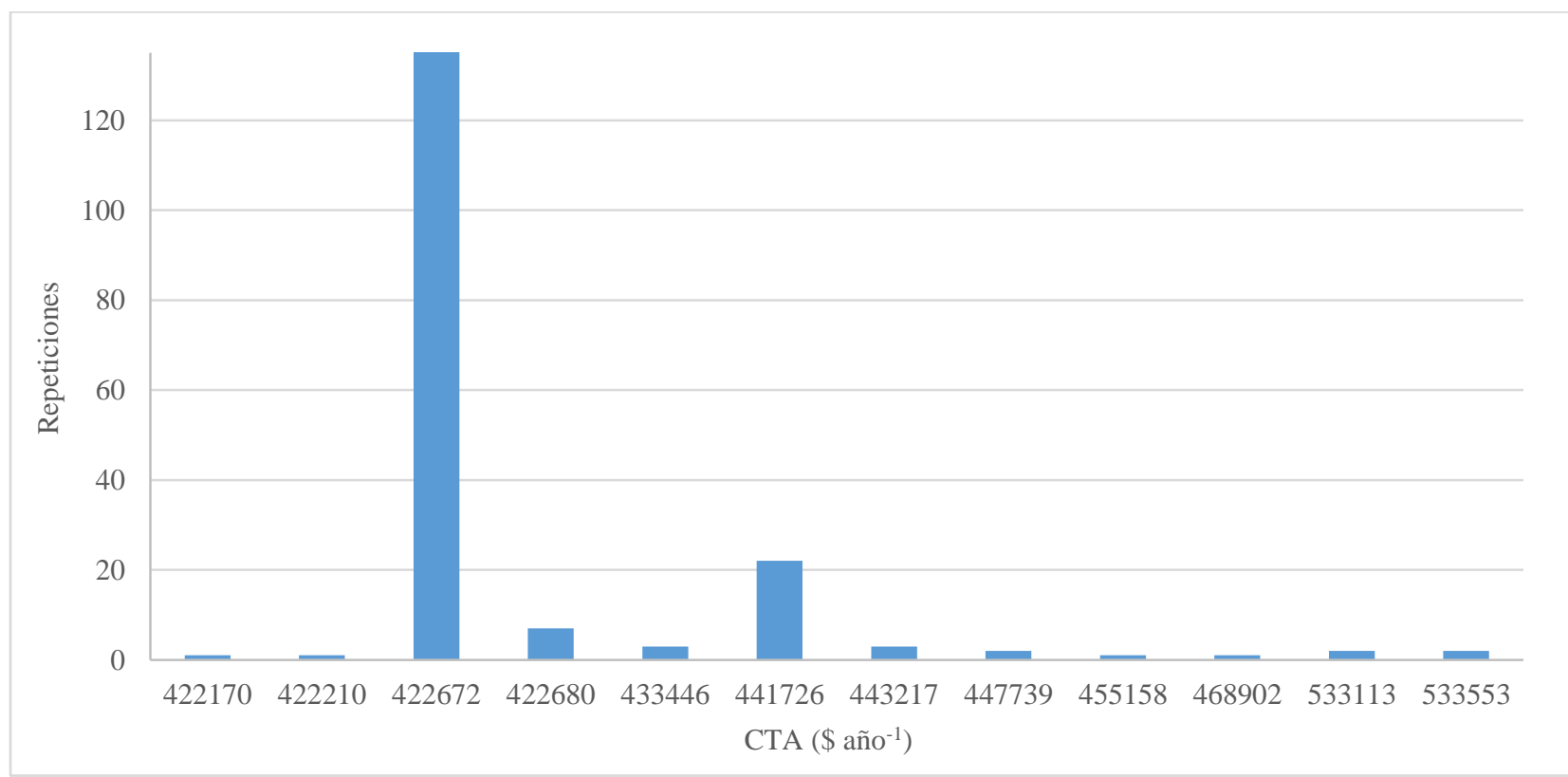

Figura 3.8. Histograma de soluciones óptimas locales obtenidas fijando 250 inicializaciones para el diseño base 3-R2.

Es posible llevar al límite el método de optimización explorando una mayor diversidad de puntos de arranque con el fin de disminuir las posibilidades de omitir soluciones óptimas locales de calidad. Bajo estas consideraciones se proponen 2000 iteraciones para el diseño 3-R2. Para el número de inicializaciones seleccionado, se incrementa el número de óptimos locales detectados 
a treinta con respecto a los doce óptimos locales definidos en la prueba con 250 iteraciones. En la Tabla 3.3 se muestran las soluciones obtenidas, donde es posible apreciar que la mayoría de los diseños simplificados tienden a ser determinados con poca frecuencia, tal es el caso de trece óptimos que sólo pudieron ser determinados en una única ocasión. Por otro lado, la mejor solución definida presenta un $\mathrm{CTA}_{\text {Paterson }}$ de $\$ 420,699.51$ coincidiendo con la mejor solución determinada en el diseño redundante 3-R1; esta solución aparece por primera vez en la iteración 1386. Los resultados de la optimización de la topología redundante 3-R2 con 2000 iteraciones resaltan los riesgos de proponer diseños base con un alto nivel de redundancia pues, aunque la variedad de óptimos locales incrementa conforme el número de equipos aumenta, las probabilidades de detectar soluciones de calidad también se pueden ver afectadas de forma negativa.

Tabla 3.3. Soluciones óptimas locales para el diseño base 3-R2 fijando 2000 inicializaciones.

\begin{tabular}{|c|c|c|c|c|c|}
\hline $\begin{array}{l}\text { CTA }_{\text {Paterson }} \\
\left(\$ \text { año }^{-1}\right)\end{array}$ & $\begin{array}{l}\text { Primera } \\
\text { Aparición }\end{array}$ & $\begin{array}{l}\text { Veces que } \\
\text { se repite }\end{array}$ & $\begin{array}{l}\text { CTA }_{\text {Paterson }} \\
\left(\$ \text { año }^{-1}\right)\end{array}$ & $\begin{array}{c}\text { Primera } \\
\text { Aparición }\end{array}$ & $\begin{array}{l}\text { Veces que } \\
\text { se repite }\end{array}$ \\
\hline 420699.51 & 1386 & 104 & 450466.42 & 1343 & 1 \\
\hline 420742.73 & 1275 & 1 & 454898.7 & 783 & 5 \\
\hline 422169.76 & 206 & 1 & 455157.66 & 90 & 3 \\
\hline 422176.28 & 589 & 1 & 458056.83 & 272 & 2 \\
\hline 422210.08 & 198 & 1 & 468901.98 & 89 & 7 \\
\hline 422259.36 & 367 & 1 & 473404.87 & 578 & 1 \\
\hline 422414.92 & 709 & 1 & 473986.6 & 548 & 2 \\
\hline 422672.46 & 3 & 1564 & 474951.11 & 1740 & 1 \\
\hline 422679.52 & 35 & 34 & 480404.02 & 1174 & 2 \\
\hline 433445.96 & 135 & 4 & 485616.96 & 995 & 3 \\
\hline 436353.19 & 576 & 1 & 506419.39 & 1686 & 1 \\
\hline 436698.56 & 559 & 3 & 531089.72 & 1391 & 1 \\
\hline 441726.28 & 4 & 211 & 533113.29 & 1 & 8 \\
\hline 443217.11 & 60 & 11 & 533552.89 & 45 & 16 \\
\hline 447738.78 & 141 & 5 & 552606.7 & 1053 & 1 \\
\hline
\end{tabular}

En la Figura 3.9 se presentan los resultados de la optimización de la red redundante 3-R3 mostrada en la Figura 3.7. Para este caso se considera una configuración que involucra una distribución más equitativa sobre las corrientes de proceso en comparación con la red redundante 3-R2. Se proponen 250 iteraciones a través de las cuales se identifican trece óptimos locales 
diferentes. Para el diseño 3-R3, a pesar de considerar un número modesto de iteraciones, es posible determinar la mejor solución contenida en las estructuras 3-R1 y 3-R2 con CTA $\mathrm{Aaterson}_{\text {de }}$ $\$ 420,699.51$ precisada en la Figura 3.5a. Sumado a esto, las diferentes interacciones entre corrientes de proceso que caracterizan a la estructura 3-R3, permiten definir un nuevo diseño óptimo local más económico que presenta un $\mathrm{CTA}_{\text {Paterson }}$ de \$418,398.36. La mejor solución se muestra en la Figura 3.10 y requiere dividir las corrientes $\mathrm{H} 2$ y $\mathrm{C} 1$ en dos sub-corrientes respectivamente, esta característica permite recuperar una mayor cantidad de calor con un total de $8,143 \mathrm{~kW}$ y disminuir el área de intercambio de calor a $11,193 \mathrm{~m}^{2}$ con respecto a la mejor solución determinada en la Sección 3.4 con CTA $_{\text {Paterson }}$ de $\$ 420,699.51$, la cual presenta valores de $8,096 \mathrm{~kW}$ y $12,439 \mathrm{~m}^{2}$ respectivamente.

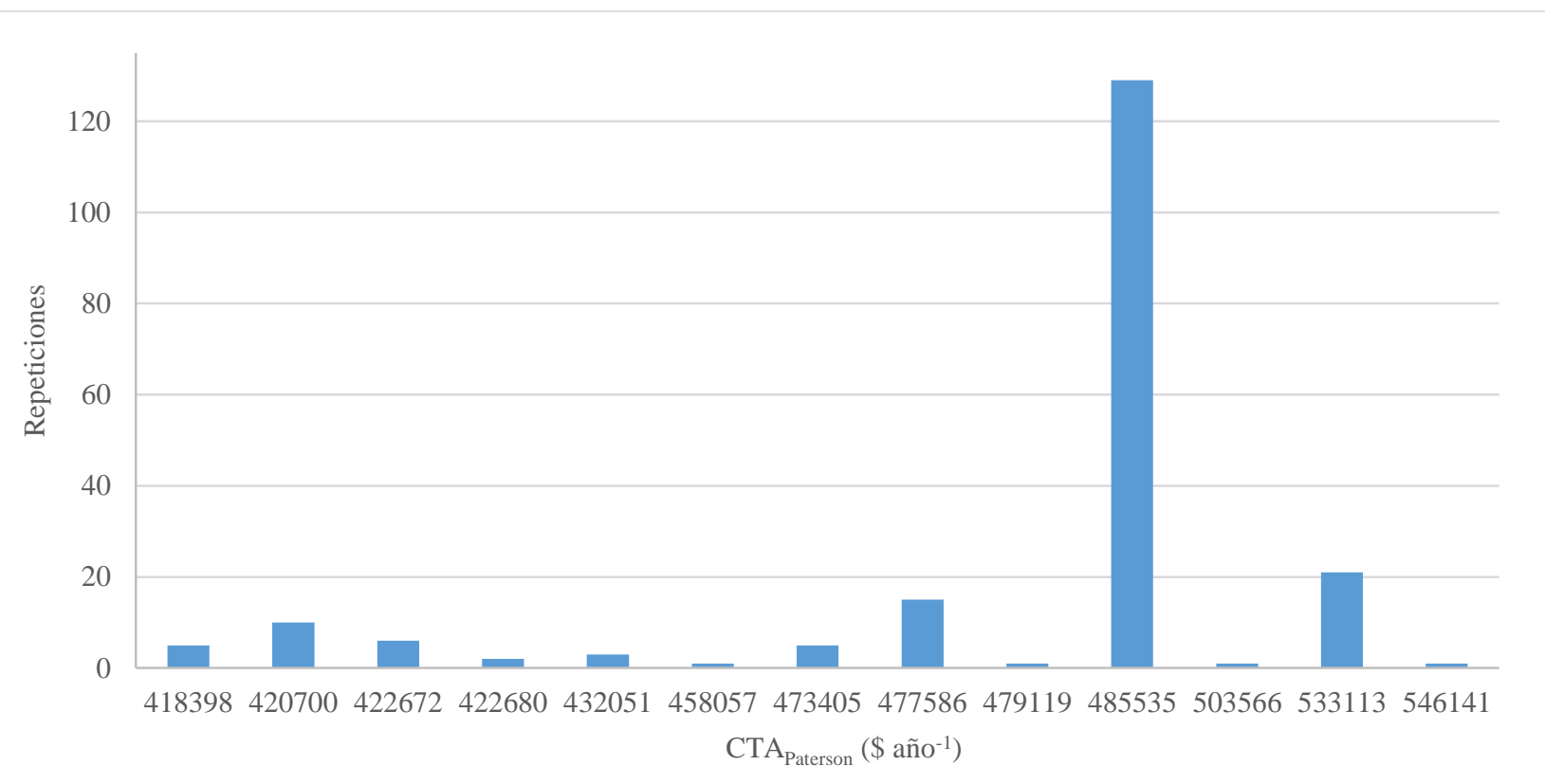

Figura 3. 9. Histograma de soluciones óptimas locales obtenidas fijando 250 inicializaciones para el diseño base 3-R3.

En resumen, un número de iteraciones considerable se traduce en una mejor evaluación de la región factible disminuyendo la posibilidad de pasar por alto soluciones óptimas locales que pudieran representar mejoras en el CTA. Los resultados discutidos proporcionan evidencia de que la capacidad de generar redes óptimas económicas pudiera recaer en gran parte en la configuración inicial de la estructura redundante, por lo que proponer diseños base con diferentes configuraciones pudiera incrementar la posibilidad de obtener óptimos locales con CTA competitivo en tiempos de cómputo razonables. 


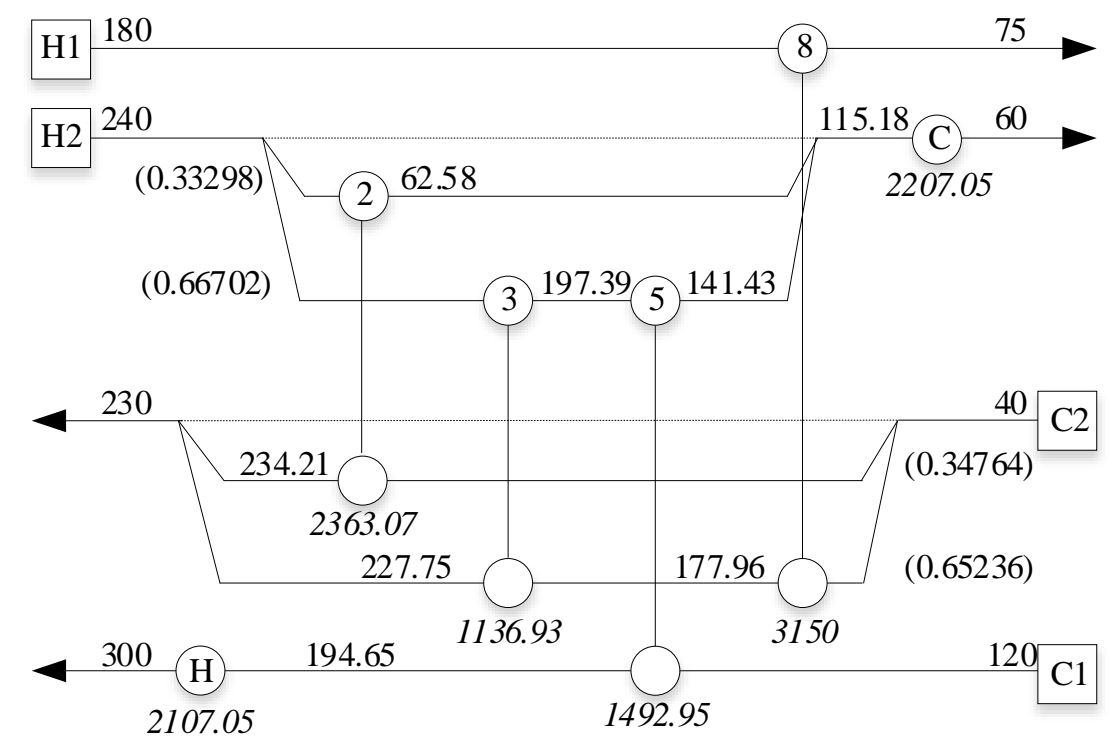

Figura 3.10. Mejor solución óptima local con $\mathrm{CTA}_{\text {Paterson }}$ de $\$ 418,398.36$ contenida en la estructura 3-R3, para el ejemplo ilustrativo 3.1 (temperaturas $\left({ }^{\circ} \mathrm{C}\right.$ ) en fuente normal, cargas térmicas $(\mathrm{kW})$ en cursivas y fracciones de flujo entre paréntesis). 


\section{Capítulo 4. Metodología secuencial para la síntesis de redes de intercambio de calor}

\subsection{Redes redundantes de intercambio de calor}

En este capítulo se propone una metodología para la síntesis de redes de intercambio de calor mediante la generación y optimización de redes redundantes la cual tiene como propósito afrontar algunas de las dificultades combinatorias que la tarea de síntesis representa. Para un problema con $\mathrm{NH}$ corrientes calientes y NC corrientes frías, sin considerar divisiones de corrientes, existen $(\mathrm{NH} \cdot \mathrm{NC})$ ! posibles estructuras (Ponton y Donaldson, 1974). Esto significa que para un caso considerado de mediana escala, donde cinco corrientes calientes y cinco corrientes frías intercambian calor, existen aproximadamente $1.55 \times 10^{25}$ combinaciones entre corrientes de proceso, dicha cantidad es mayor que el número estimado de estrellas en el espacio observable (Dokkum y Conroy, 2010). Aunque este cálculo no implica que las estructuras deban ser necesariamente factibles, proporciona un panorama de la vasta cantidad de diseños de red que pueden ser obtenidos.

En los enfoques de programación entera mixta (secuenciales o simultáneos), el uso de variables binarias para determinar los apareamientos entre corrientes de proceso implica el crecimiento exponencial del tamaño del espacio de búsqueda lo que normalmente conduce a una explosión combinatoria, incluso en problemas de mediana escala. Con la optimización redes redundantes de intercambio de calor se busca determinar un dominio factible representado por un número sobreestimado pero realista de intercambiadores de calor que disminuya la carga combinatoria del problema de síntesis, acotando la diversidad de diseños óptimos a aquellos que sólo están contenidos en topologías redundantes previamente establecidas.

Las topologías redundantes presentan la posibilidad de ser reducidas omitiendo el uso de variables binarias mediante técnicas de PNL para obtener diseños de redes de intercambio de calor, no obstante, es necesario proponer un esquema que permita generar dichas estructuras 
sobreestimadas de forma automatizada con la certeza de que presenten un dominio factible para garantizar la obtención de diseños finales óptimos mediante el algoritmo de optimización descrito en el Capítulo 3.

\subsection{Distribución de cargas térmicas para la generación de topologías redundantes}

En este capítulo se investiga la posibilidad de generar diseños de red mediante el problema de uso mínimo de servicios auxiliares (Cerdá y col., 1983), retomando la notación y el planteamiento del modelo de transporte del Capítulo 2. La solución del modelo de transporte determina cargas térmicas $\left(q_{i, l, j, l 1}, q h u_{j, l 1}\right.$ y $\left.q c u_{i, l}\right)$ diferentes de cero, que pueden ser vistos como apareamientos prometedores factibles entre corrientes de proceso y servicios auxiliares para definir las metas de energía. Debido a que con el problema de uso mínimo de servicios auxiliares no se pretende minimizar explícitamente el costo de una red de intercambio de calor, generalmente otorga un número considerable de apareamientos, por lo que es viable proponer reglas que permitan interpretar los intercambios de calor como estructuras de red redundantes.

La dificultad de construir topologías redundantes bajo el esquema de uso mínimo de servicios auxiliares proviene de que la distribución de cargas térmicas determinada por el modelo de transporte no exhibe de manera evidente ni simple una estructura de red para la recuperación de calor. Sumado a esto, se busca diseñar redes redundantes que contengan una variedad de opciones en cuanto a estructuras de redes de intercambio de calor se refiere, como son aquellas con división de corrientes, que incrementen las posibilidades de obtener redes de intercambio de calor con CTA competitivo al ser simplificadas.

Para ubicar las dificultades que pudieran surgir de la transición entre los intercambios de calor obtenidos de la solución del modelo de transporte y una red redundante se retoma el ejemplo ilustrativo 3.1 de la Sección 3.3. Los datos de corrientes y costos del caso de estudio introducido a la literatura por Zamora y Grossmann (1997), que comprende dos corrientes de proceso calientes y dos frías es descrito nuevamente en la Tabla 4.1 y renombrado como ejemplo ilustrativo 4.1 . 
Tabla 4.1. Datos de corrientes y costos del ejemplo ilustrativo 4.1.

\begin{tabular}{|c|c|c|c|c|}
\hline Corriente & $\begin{array}{c}\text { Temperatura de } \\
\text { suministro } \\
\operatorname{Ts}\left({ }^{\circ} \mathrm{C}\right)\end{array}$ & $\begin{array}{c}\text { Temperatura } \\
\text { objetivo } \\
\text { To }\left({ }^{\circ} \mathrm{C}\right)\end{array}$ & $\begin{array}{c}\text { Flujo de capacidad } \\
\text { calorífica } \\
\mathrm{F}\left(\mathrm{kW}^{\circ} \mathrm{C}^{-1}\right)\end{array}$ & $\begin{array}{l}\text { Coeficiente de } \\
\text { película } \\
\mathrm{h}\left(\mathrm{kW} \mathrm{m}^{-1}{ }^{\circ} \mathrm{C}^{-1}\right)\end{array}$ \\
\hline H1 & 180 & 75 & 30 & 0.15 \\
\hline $\mathrm{H} 2$ & 240 & 60 & 40 & 0.10 \\
\hline $\mathrm{C} 1$ & 40 & 230 & 35 & 0.20 \\
\hline $\mathrm{C} 2$ & 120 & 300 & 20 & 0.10 \\
\hline HU & 325 & 325 & - & 2 \\
\hline $\mathrm{CU}$ & 25 & 40 & - & 0.50 \\
\hline \multicolumn{5}{|c|}{$\begin{array}{l}\text { Costo de intercambiadores de calor y enfriadores }\left(\$ \mathrm{año}^{-1}\right) \\
\text { Costo de calentadores }\left(\$ \mathrm{año}^{-1}\right)=1500+60\left[\text { Área }\left(\mathrm{m}^{2}\right)\right]^{0.8} \\
\text { Costo de servicios de calentamiento }=110\left(\$ \mathrm{~kW}^{-1} \mathrm{año}^{-1}\right) \\
\text { Costo de servicios de enfriamiento }=10\left(\$ \mathrm{~kW}^{-1} \mathrm{año}^{-1}\right)\end{array}$} \\
\hline
\end{tabular}

Tabla 4.2. Intercambios de calor obtenidos de la solución del modelo de transporte.

\begin{tabular}{ccccc}
\hline$i$ & $l$ & $j$ & $l l$ & $\begin{array}{c}q \\
(\mathrm{~kW})\end{array}$ \\
\hline $\mathrm{qhu}$ & - & 2 & 1 & 1400 \\
$\mathrm{qhu}$ & - & 2 & 2 & 900 \\
\hline 1 & 3 & 1 & 3 & 500 \\
1 & 4 & 1 & 4 & 1650 \\
1 & 3 & 2 & 3 & 1000 \\
2 & 2 & 1 & 2 & 2100 \\
2 & 3 & 1 & 3 & 1250 \\
2 & 3 & 1 & 4 & 275 \\
2 & 4 & 1 & 6 & 350 \\
2 & 5 & 1 & 5 & 525 \\
2 & 2 & 2 & 2 & 300 \\
\hline 2 & 3 & $\mathrm{qcu}$ & - & 475 \\
2 & 4 & $\mathrm{qcu}$ & - & 1850 \\
2 & 5 & $\mathrm{qcu}$ & - & 75 \\
\hline
\end{tabular}

En la Tabla 4.2 se describe la tabla de intercambios de calor obtenida de la solución del modelo de transporte para un HRAT de $10{ }^{\circ} \mathrm{C}$. Para resolver el modelo de transporte se utilizó GAMS 21.4 con CPLEX v.12 como resolvedor de PL. En resumen, las metas de energía para un HRAT de $10{ }^{\circ} \mathrm{C}$ imponen que es posible recuperar un máximo de $7950 \mathrm{~kW}$ requiriendo $2300 \mathrm{~kW}$ como 
servicios de calentamiento y $2400 \mathrm{~kW}$ como de servicios de enfriamiento. Se puede apreciar que la solución del modelo de transporte indica que únicamente son necesarios servicios de calentamiento en la corriente $\mathrm{C} 2$ y servicios de enfriamiento en la corriente $\mathrm{H} 2$.

La distribución de cargas térmicas de la Tabla 4.2 representa en cada fila un intercambio de calor o apareamiento, pudiéndose interpretar de la siguiente forma. Cierta corriente caliente $i \in I$ desde el intervalo de temperatura $l \in L$ o un servicio de calentamiento, suministra una cantidad de energía hacia una cierta corriente fría $j \in J$ en el intervalo de temperatura $l 1 \in L 1$ o hacia un servicio de enfriamiento.

En la Figura 4.1 se muestra la representación gráfica de los posibles apareamientos entre las corrientes de proceso y servicios auxiliares, mientras que en la Figura 4.2 se muestran los intercambios de calor que se mantienen después de resolver el modelo de transporte. Es posible diferenciar que el número de interacciones es reducido considerablemente; de las 39 posibles rutas de intercambio de calor iniciales, únicamente 14 son seleccionados por el modelo de PL. Cabe mencionar que la solución del modelo de transporte no es estrictamente única y pueden existir otras configuraciones que cumplan con la misma meta de recuperación de energía con número diferente de apareamientos. Esto depende de la llamada solución básica inicial y del algoritmo de solución, no obstante, la exploración de las múltiples soluciones óptimas en resolvedores comerciales no es una tarea fácil de implementar y, por otro lado, el desarrollo de algoritmos para la solución del modelo de transporte no forma parte de los objetivos de este trabajo.
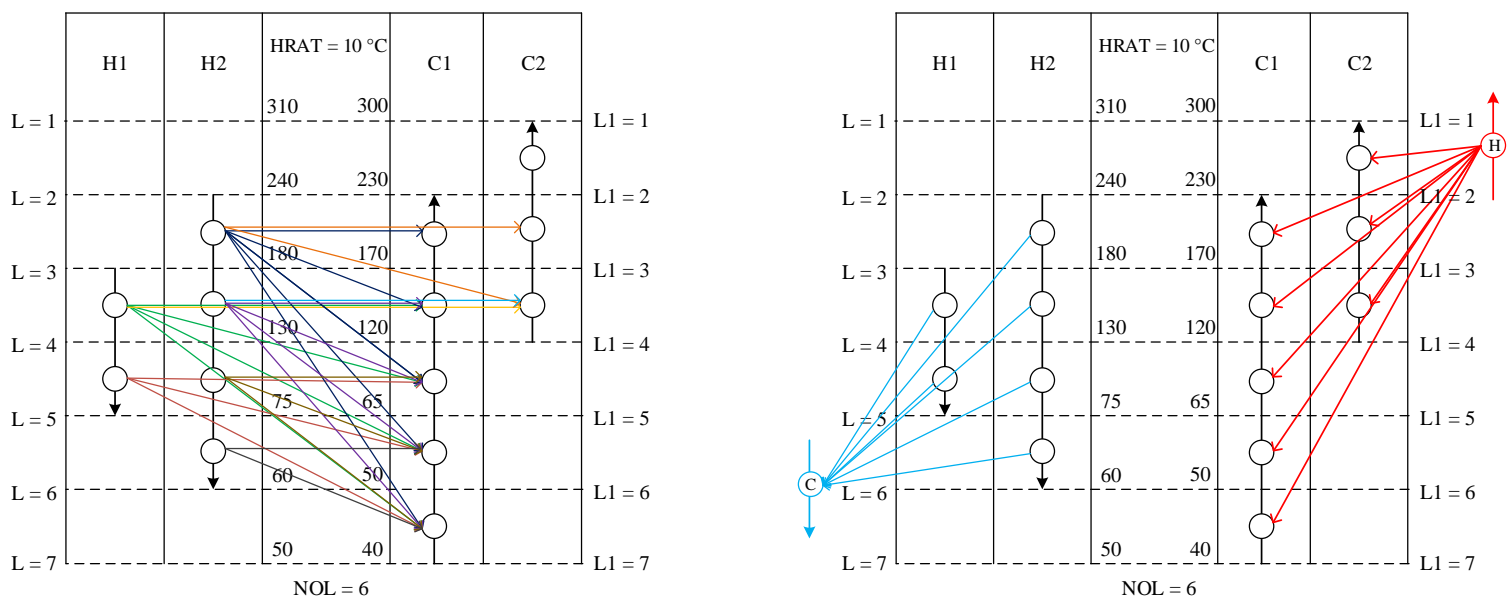

Figura 4.1. Rutas para el intercambio de calor entre corrientes de proceso y servicios auxiliares. 


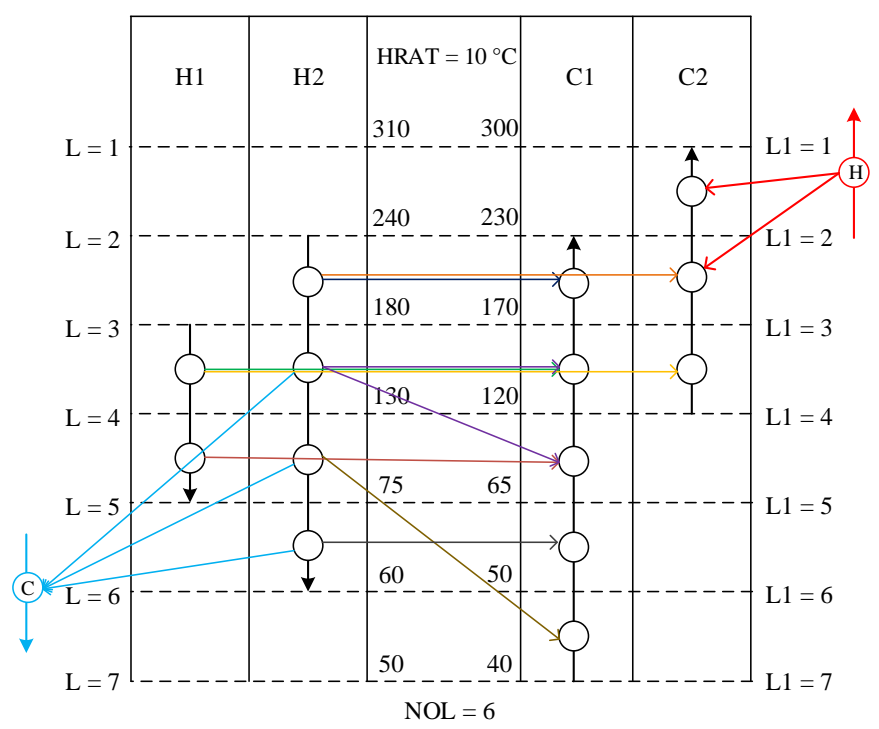

Figura 4.2. Representación de la distribución de cargas térmicas.

Un primer acercamiento para la generación de redes redundantes de intercambio de calor mediante la formulación del problema de uso mínimo de servicios auxiliares es proponer diseños base sin división de corrientes. Esta suposición suele hacerse con el propósito de evitar posibles complejidades, al reducir el espacio de solución. En la Figura 4.3 se muestra una estructura de red sin división de corrientes que considera cada elemento de la Tabla 4.2 como un intercambiador de calor, los equipos están acomodados de tal forma que corresponden al mismo orden de la tabla, siendo el primer elemento el primer intercambiador de calor y así sucesivamente.

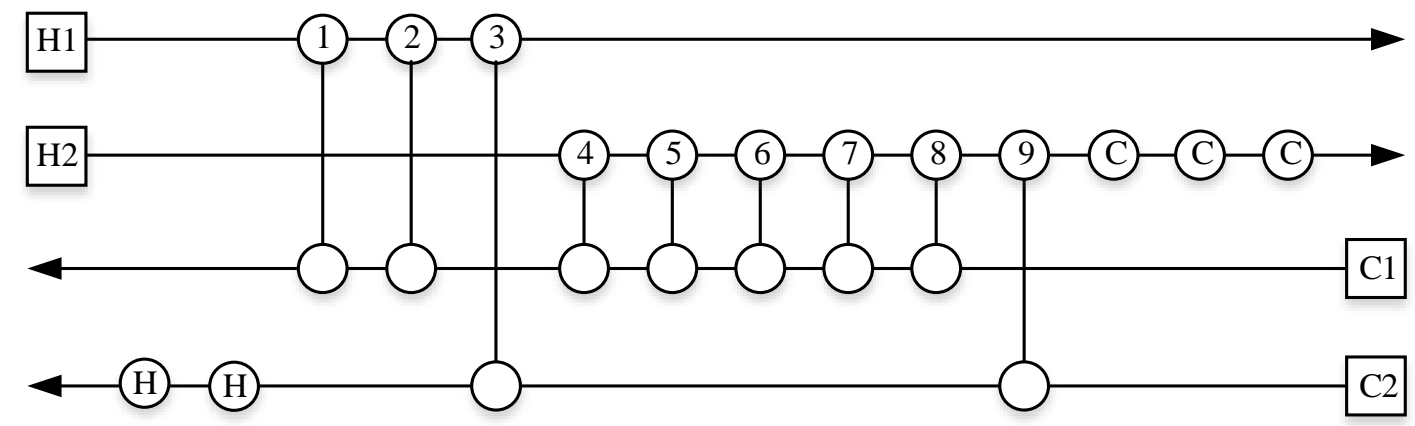

Figura 4.3. Diseño de red redundante sin división de corrientes, basado en los intercambios de calor de la solución del modelo de transporte.

La red redundante descrita en la Figura 4.3 pudiera no ser idónea como un diseño base para la optimización, algunos de los inconvenientes sobresalen de forma evidente como son las 
interacciones entre corrientes que se repiten consecutivamente (e.g. equipos 4, 5, 6, 7, y 8). Estos reapareamientos representan una carga para el modelo de optimización pues es posible anticipar que un sólo equipo puede realizar la misma labor de recuperación de calor involucrando un único cargo fijo, por lo que significan un gasto innecesario en términos del consumo de recursos computacionales y también limitan la variedad de soluciones óptimas locales. El origen de los problemas señalados pudiera ser que no se ha tomado a consideración ningún criterio razonable para la colocación de equipos, lo que pudiera generar redes redundantes poco eficientes.

Las topologías redundantes propuestas no sólo deben poseer características que aseguren la factibilidad del espacio de solución, sino que también resultaría oportuno que los intercambiadores de calor puedan ser colocados de forma estratégica favoreciendo la tarea de síntesis de redes de intercambio de calor. La distribución de cargas térmicas no sólo concede los apareamientos entre las corrientes de proceso y servicios auxiliares, también indica desde qué niveles de temperatura se debe intercambiar calor. Los intervalos de temperatura pueden determinar el orden para la asignación de equipos dando prioridad a aquellos que se encuentran en un nivel de temperatura alto, seguido por los apareamientos ubicados en niveles inferiores.

Con los elementos descritos, otra alternativa que no termina de resolver los problemas que surgen de planteamiento de la generación de topologías redundantes, consiste en estructurar una representación por etapas de la distribución de cargas térmicas sobre un diagrama de malla sin división de corrientes, donde cada etapa se asocia a un intervalo de temperatura. En la Figura 4.4 se propone una posible red redundante para el ejemplo ilustrativo 4.1, en donde se puede distinguir el arreglo por etapas y el ordenamiento con respecto a los intervalos de temperatura. De esta manera la primera etapa contiene todos los elementos de la Tabla 4.2 donde las corrientes calientes o servicios de enfriamiento estén definidos en el intervalo de temperatura caliente $l=1$ y los elementos en el intervalo de temperatura fría $l 1=1$ en el caso de las corrientes frías o servicios de calentamiento. De forma análoga sucede con las etapas posteriores donde se asocian respectivamente los elementos cada intervalo de temperatura.

El diseño de red planteado en la Figura 4.4 presenta una distribución de equipos sobre el diagrama de malla que evita en gran medida los apareamientos consecutivos entre corrientes de proceso, dando la posibilidad de que exista una mayor variedad de soluciones óptimas locales. Por otro lado, algunos inconvenientes que surgen de esta propuesta deben ser señalados, por 
ejemplo, en la etapa dos, la corriente caliente $\mathrm{H} 2$ admite un calentador $\left(q h u_{2,2}\right)$ y los recuperadores de calor $1\left(q_{2,2,1,2}\right)$ y $2\left(q_{2,2,2,2}\right)$, sin embargo, los tres equipos poseen exactamente la misma jerarquía debido a que intercambian calor a lo largo de los mismos intervalos de temperatura calientes y fríos, esto implica que el diseñador debe tomar una decisión arbitraria sobre cual equipo debe ser colocado primero, de forma similar sucede en los intervalos posteriores. En adición, el aspecto crítico se produce debido al equipo $8\left(q_{2,4,1,6}\right)$, en donde la corriente $\mathrm{H} 2$ suministra calor desde el cuarto intervalo de temperatura caliente hasta el sexto intervalo de temperatura de la corriente fría $\mathrm{C} 1$, produciendo un intercambio de calor que cruza los intervalos de temperatura dejando al equipo $9\left(q_{2,5,2,5}\right)$ en medio de este intercambio de calor. Los cruces de temperaturas pudieran limitar en gran medida la cantidad posible de energía a recuperar por lo que no suelen ser incluidos en la mayoría de las metodologías de síntesis.

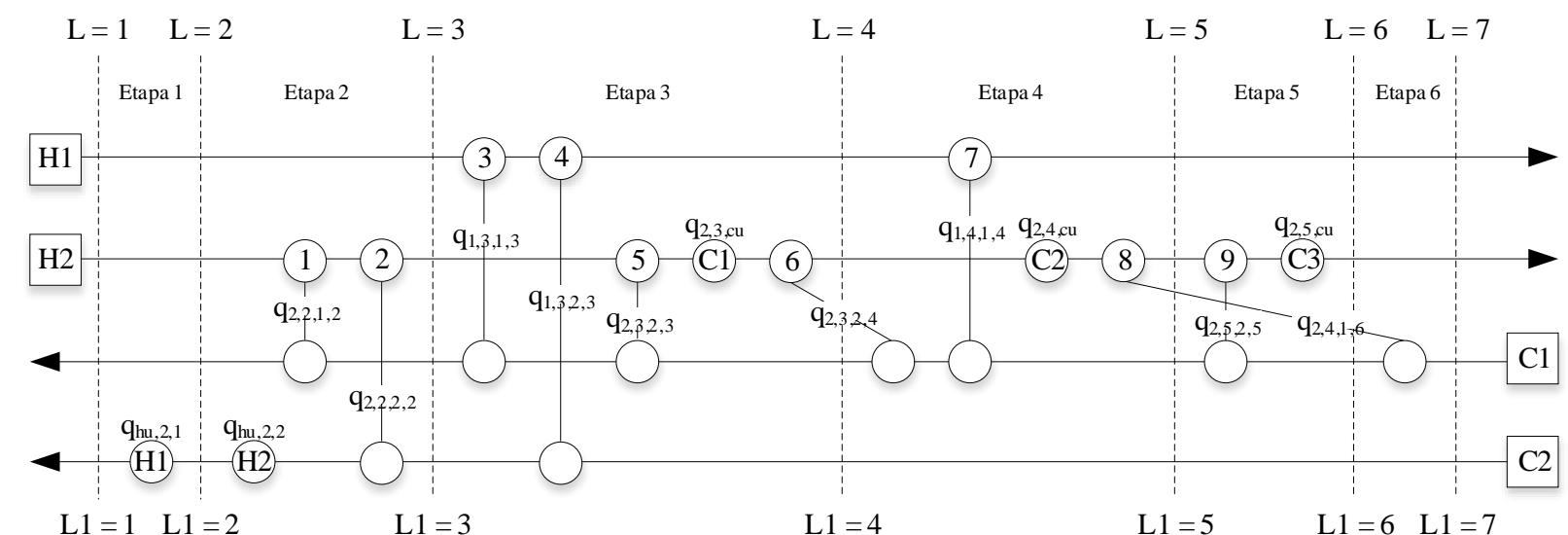

Figura 4.4. Topología redundante sin división de corrientes, basada en intervalos de temperatura.

Las propuestas de red desarrolladas en las Figuras 4.3 y 4.4, resaltan las dificultades que surgen al diseñar estructuras redundantes sin división de corrientes a partir de un conjunto de apareamientos que no sugieren la forma en que se deben distribuir los intercambiadores de calor sobre las corrientes de proceso. Es posible suponer que dichos conflictos incrementarán drásticamente en problemas con un mayor número de corrientes, por lo que estos planteamientos resultan poco fiables. Es esencial determinar estructuras de red redundantes que no estén restringidas a decisiones arbitrarias ni que representen un impedimento en la determinación de diseños finales con CTA competitivo.

Para superar los problemas antes mencionados se propone el uso de divisores y mezcladores de corrientes. El concepto consiste en la posibilidad de dividir una corriente de proceso si existe 
más de un intercambio de calor sobre un mismo intervalo de temperatura, lo que permite acomodar equipos sobre diferentes ramales de una misma corriente de proceso y a su vez conserven la misma jerarquía al tener un arreglo en paralelo.

En adición, admitir divisiones de corrientes puede incrementar la capacidad de recuperación de energía lo que supone una mayor posibilidad de obtener redes de intercambio de calor de bajo costo, sin embargo, se debe evitar el caso extremo, donde cada intercambiador de calor suponga establecer una sub-corriente de proceso diferente. Es necesario desarrollar técnicas que permitan generar estructuras redundantes eficientes guardando un compromiso conveniente entre arreglos en serie y en paralelo cuando sea necesario.

\subsection{Generación de redes redundantes eficientes}

En esta sección se proponen reglas que permiten generar topologías redundantes que incluyan divisiones de corrientes en caso de ser necesario para evitar conflictos que se producen al momento en el que dos equipos presenten la misma jerarquía. El desarrollo de estas estructuras pretende hacer uso de la tabla de distribución de cargas térmicas, y en específico de los intervalos de temperatura para la determinación sistemática de sub-corrientes de proceso necesarias, y la distribución de los equipos sobre estas.

Para los propósitos de este trabajo, se considera la simplificación de admitir a lo más un servicio auxiliar extremo por cada corriente de proceso. La solución del modelo de transporte indica las corrientes que requieren servicios auxiliares en diferentes intervalos de temperaturas. Sin embargo, un sólo calentador o enfriador será colocado al final de la corriente según indique la solución del modelo de transporte, omitiendo el uso de los intervalos de temperatura para estos casos. En problemas con un número considerable de corrientes de proceso, la solución el modelo de transporte pudiera indicar que son necesarios servicios auxiliares a lo largo de varios intervalos de temperatura de una corriente de proceso, lo que se traduce a numerosos calentadores y enfriadores intermedios; el imponer el límite de uno por corriente en topologías redundantes, previene que el modelo de optimización caiga en óptimos locales con un uso excesivo de servicios auxiliares que se traduzcan en soluciones poco convenientes en términos de costos. Cabe destacar que dicha simplificación no es necesariamente un criterio decisivo para 
la síntesis de redes de intercambio de calor con bajo costo, Ponce-Ortega y col. (2010) formulan un modelo de PNL-EM para la síntesis de redes de intercambio donde discuten que en ciertos casos es posible disminuir el costo del diseño final con un posicionamiento óptimo de los servicios auxiliares en niveles intermedios.

\section{Topología redundante 1}

Si se considera que la distribución de cargas térmicas provee de un criterio aceptable para el ordenamiento de los equipos sobre un diseño de red, es pertinente reordenar la Tabla 4.2 de modo que el primer elemento de la tabla corresponda en la topología redundante, al primer equipo de intercambio de calor sobre la estructura de red redundante a generar. Como se mostró en la Sección 4.2 las etapas de un diagrama pueden ser asociadas a los intervalos de temperatura, siendo las primeras etapas las que se encuentran en un nivel de temperatura superior. Por esta razón en la Tabla 4.3 se reordena la distribución de cargas térmicas con respecto a los intervalos de temperatura calientes (en negritas); adicionalmente se agrega una columna a la izquierda donde se asigna una numeración a cada fila que se relaciona con un respectivo intercambiador de calor, IC.

Tabla 4.3. Distribución de cargas térmicas ordenada con respecto a los intervalos de temperatura calientes.

\begin{tabular}{cccccc}
\hline IC & $i$ & $\hat{\boldsymbol{l}}$ & $j$ & $l l$ & $\begin{array}{c}q \\
\mathrm{~kW})\end{array}$ \\
\hline 1 & $\mathrm{qhu}$ & - & 2 & - & 2300 \\
\hline 2 & 2 & $\mathbf{2}$ & 1 & 2 & 2100 \\
3 & 2 & $\mathbf{2}$ & 2 & 2 & 300 \\
4 & 1 & $\mathbf{3}$ & 1 & 3 & 500 \\
5 & 1 & $\mathbf{3}$ & 2 & 3 & 1000 \\
6 & 2 & $\mathbf{3}$ & 1 & 3 & 1250 \\
7 & 2 & $\mathbf{3}$ & 1 & 4 & 275 \\
8 & 1 & $\mathbf{4}$ & 1 & 4 & 1650 \\
9 & 2 & $\mathbf{4}$ & 1 & 6 & 350 \\
10 & 2 & $\mathbf{5}$ & 1 & 5 & 525 \\
\hline 11 & 2 & - & $\mathrm{qcu}$ & - & 2400 \\
\hline
\end{tabular}


La distribución de cargas térmicas demanda cruces entre intervalos de temperatura cuando una corriente caliente suministra calor desde un intervalo caliente superior a una corriente fría en un intervalo de temperatura de nivel inferior, en un diagrama de malla sin división de corrientes estos cruces se interpretaron como una línea diagonal, lo que no es usual en la representación de redes de intercambio de calor. Por esta razón, se propone definir un esquema para la colocación de equipos por separado para las corrientes de proceso calientes y para las corrientes de proceso frías que permitan ubicar adecuadamente el posicionamiento de los intercambiadores de calor sobre las debidas sub-corrientes de proceso cuando dos o más intercambios de calor se encuentran sobre el mismo intervalo de temperatura.

Se entenderá que dos equipos presentan la misma jerarquía si en la Tabla 4.3 se encuentran sobre el mismo intervalo de temperatura. En la Tabla 4.4 se sitúan los intercambios de calor considerando únicamente las corrientes de proceso calientes, omitiendo lo intercambios que representan servicios auxiliares y tomando en cuenta el orden de equipos asociado a la Tabla 4.3. Sobre cada etapa se colocan paralelamente todos los intercambiadores de calor que corresponden a un respectivo intervalo de temperatura, por lo que cada fila de la tabla representa en un principio una potencial sub-corriente para la corriente de proceso indicada en la primera columna.

Tabla 4.4. Representación inicial por etapas de equipos en las corrientes calientes.

\begin{tabular}{|c|c|c|c|c|c|c|}
\hline $\begin{array}{c}\text { Corriente } \\
i\end{array}$ & $\begin{array}{c}\text { Etapa } 1 \\
l=1\end{array}$ & $\begin{array}{c}\text { Etapa 2 } \\
l=2\end{array}$ & $\begin{array}{c}\text { Etapa 3 } \\
l=3\end{array}$ & $\begin{array}{c}\text { Etapa } 4 \\
l=4\end{array}$ & $\begin{array}{c}\text { Etapa 5 } \\
l=5\end{array}$ & $\begin{array}{c}\text { Etapa 6 } \\
l=6\end{array}$ \\
\hline $\mathrm{H} 2$ & & 2 & & & & \\
\hline $\mathrm{H} 2$ & & 3 & & & & \\
\hline $\mathrm{H} 1$ & & & 4 & & & \\
\hline $\mathrm{H} 1$ & & & 5 & & & \\
\hline $\mathrm{H} 2$ & & & 6 & & & \\
\hline $\mathrm{H} 2$ & & & $(7$ & & & \\
\hline $\mathrm{H} 1$ & & & & 8 & & \\
\hline $\mathrm{H} 2$ & & & & 9 & & \\
\hline $\mathrm{H} 2$ & & & & & \\
\hline
\end{tabular}


Con el fin de diseñar topologías redundantes que contengan estructuras con características que incluyen tanto arreglos en serie como en paralelo se busca reducir el uso excesivo de divisiones de corrientes. Para esto se comparan los intercambios que pertenecen a una misma corriente intentando colocar, si los intervalos de temperatura lo permiten, tantos equipos en serie como sea posible. A continuación, se ejemplifica de forma detallada un conjunto de pautas para la generación de una red redundante con las características descritas.

El procedimiento consiste en un análisis comparativo por filas de la Tabla 4.4, comenzando por la fila superior, con cada fila que se encuentre por debajo de la misma. Se admite colocar equipos en serie sobre la fila superior, si la fila inferior comparada pertenece a la misma corriente de proceso y presenta un equipo en una etapa posterior (de menor nivel de temperatura) en donde no haya presente algún intercambiador de calor. Si alguna fila no dispone de al menos un intercambiador de calor, es posible eliminar dicha fila, eliminando a su vez la división de corriente que representaba.

En la Tabla 4.4 correspondiente al ejemplo ilustrativo 4.1, el análisis comienza sobre la primera fila que contiene al intercambiador de calor 2 en la etapa 2. En la Figura 4.5 se muestra de forma aislada la forma en que se procede a la comparación de la primera fila señalada con una flecha sólida a la izquierda, con la segunda fila señalada con una flecha blanca la cual contiene al intercambiador de calor 3 en la etapa 2. Ambas filas corresponden a la corriente de proceso caliente $\mathrm{H} 2$, pero los equipos que asocian se ubican sobre una misma etapa, por lo que se asume que poseen la misma jerarquía. Dicho esto, los equipos correspondientes deben permanecer sobre sub-corrientes de proceso diferentes.

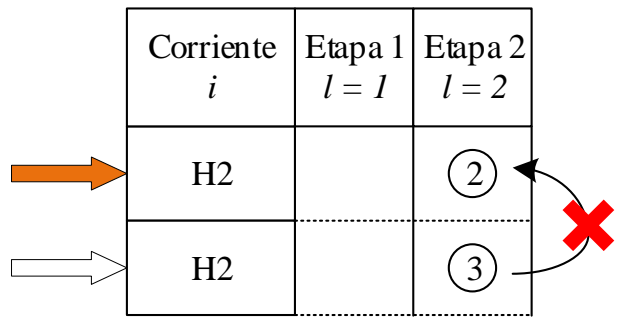

Figura 4.5. Dos filas comparadas que pertenecen a la misma corriente. Si los equipos se encuentran sobre la misma etapa deben ser colocados en sub-corrientes diferentes.

La comparación de la primera fila que corresponde a la corriente H2 continúa con las siguientes filas hasta concluir con el contenido de la tabla. Las filas 3 y 4 de la Tabla 4.4 pertenecen a la corriente de proceso H1 por lo que no hay posibilidad de colocar los equipos correspondientes en 
serie. En la Figura 4.6 se muestra la sección de la tabla en donde actualmente se realiza la comparación entre la primera y la quinta fila, ambos elementos cumplen con el criterio de pertenecer a la misma corriente de proceso y por otro lado la fila inferior señalada con la flecha blanca contiene un intercambiador de calor en la etapa 3 , que resulta ser una etapa posterior a la etapa que contiene la fila bajo análisis señalada con la flecha sólida. Dicho esto, el equipo 6 se coloca en la etapa correspondiente sobre la primera fila, pasando la quinta fila a no contener ningún intercambiador de calor asociado, por lo que se elimina la fila y la posible sub-corriente de proceso asociada a ella.

Figura 4.6. Equipo en fila inferior puede ser colocado en serie si se encuentra en una etapa posterior al último equipo de la fila bajo análisis.

\begin{tabular}{|c|c|c|c|}
\hline $\begin{array}{c}\text { Corriente } \\
i\end{array}$ & $\begin{array}{c}\text { Etapa 1 } \\
l=1\end{array}$ & $\begin{array}{c}\text { Etapa 2 } \\
l=2\end{array}$ & $\begin{array}{c}\text { Etapa 3 } \\
l=3\end{array}$ \\
\hline $\mathrm{H} 2$ & & 2 & 6 \\
\hline $\mathrm{H} 2$ & & 3 & \\
\hline $\mathrm{H} 1$ & & & 6 \\
\hline $\mathrm{H} 1$ & & & 6 \\
\hline $\mathrm{H} 2$ & & & 6 \\
\hline
\end{tabular}

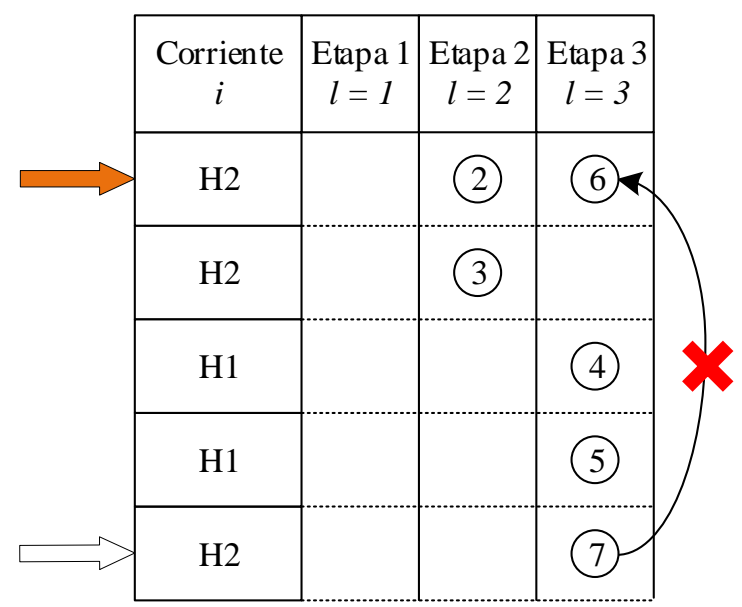

Figura 4.7. La primera fila no permite equipos sobre las etapas 2 y 3 al contener un intercambiador de calor respectivamente.

El análisis comparativo sobre la primera fila continúa, conteniendo ahora a los intercambios de calor 2 y 6 en la segunda y tercera etapa respectivamente. Se busca la posibilidad de colocar en serie otros equipos que se encuentren en las filas siguientes que no se superpongan con las etapas 
que ya contienen un equipo en la fila marcada con la flecha sólida. En la Figura 4.7 se observa que el equipo 7 de la fila siguiente marcada con la flecha blanca, no puede ser colocado en serie en sobre la primera fila puesto que el equipo 6 ya ocupa la etapa 3.

Siguiendo el análisis de la primera fila es posible concluir que los intercambios de calor 9 y 10 se pueden colocar en serie al pertenecer a la misma corriente de proceso $\mathrm{H} 2$ y estar presentes en etapas que no han sido ocupadas previamente sobre dicha fila.

Una vez concluida la comparación de la primera fila con todas las filas siguientes, se procede a realizar el mismo análisis comparativo sobre cada una de las subsecuentes filas que no han sido eliminadas. Dicho lo anterior, se procede ahora sobre la segunda fila, marcada con la flecha sólida en la Figura 4.8, la cual contiene al intercambiador de calor 3 que corresponde a la corriente $\mathrm{H} 2$, las dos filas siguientes corresponden a la corriente de proceso $\mathrm{H} 1$ por lo que no es posible colocar equipos en serie; al comparar la fila marcada con la flecha blanca se encuentra el equipo 7 sobre la etapa 3 que tampoco pudo ser colocado en serie en el análisis de la primera fila. La fila en comparación marcada con la flecha sólida no presenta ningún equipo sobre la etapa 3 por lo que se propone colocar el intercambiador de calor 7 en serie sobre la etapa correspondiente de la fila marcada con la flecha sólida indicando que los equipos son puestos en serie sobre una segunda sub-corriente de proceso. De la misma manera, la fila marcada con la flecha blanca ya no presenta ningún equipo por lo que es posible eliminarla.

Figura 4.8. $\quad$ Equipos $3 \quad$ y $\quad 7$ correspondientes a $\mathrm{H} 2$, no fueron admitidos en etapas de la primera fila, por lo que son colocados en serie sobre una sub-corriente diferente.

\begin{tabular}{|c|c|c|c|c|c|}
\hline $\begin{array}{c}\text { Corriente } \\
i\end{array}$ & $\begin{array}{c}\text { Etapa } 1 \\
l=1\end{array}$ & $\begin{array}{c}\text { Etapa } 2 \\
l=2\end{array}$ & $\begin{array}{c}\text { Etapa 3 } \\
l=3\end{array}$ & $\begin{array}{c}\text { Etapa } 4 \\
l=4\end{array}$ & $\begin{array}{c}\text { Etapa } 5 \\
l=5\end{array}$ \\
\hline $\mathrm{H} 2$ & & 2 & 6 & 9 & (10) \\
\hline $\mathrm{H} 2$ & & $(3)$ & $(7)$ & & \\
\hline $\mathrm{H} 1$ & & & 4 & & \\
\hline $\mathrm{H} 1$ & & & 5 & & \\
\hline $\mathrm{H} 2$ & & & 7 & & \\
\hline $\mathrm{H} 1$ & & & & 8 & \\
\hline
\end{tabular}

El análisis de la fila marcada con la flecha sólida concluye al no haber más equipos que 
involucren a la corriente de proceso $\mathrm{H} 2$. El procedimiento se repite con las filas siguientes colocando equipos en serie cuando las filas comparadas correspondan a la misma corriente de proceso y la fila inferior (flecha blanca) contenga un equipo en una etapa posterior en comparación con la última etapa ocupada por algún equipo de la fila superior (flecha sólida). El análisis se concluye al llegar a la última fila no eliminada para lo cual se han colocado tantos equipos en serie cómo es posible y en paralelo cuando dos equipos presentan la misma jerarquía. Adicionalmente se han eliminado un cierto número de filas en comparación con la Tabla 4.4, de modo que las filas resultantes representan a las sub-corrientes de proceso calientes que dispondrá el diseño de red y los equipos que se deben colocar sobre estas. En la Tabla 4.5 se ilustra el diagrama al final del procedimiento, donde se ha concluido el análisis comparativo para las corrientes calientes y se ha reducido el número de filas de nueve a cuatro en comparación con la Tabla 4.4. Se puede apreciar que dos filas corresponden a $\mathrm{H} 2$ y dos a $\mathrm{H} 1$ lo que implica que ambas corrientes de proceso deben dividirse en dos sub-corrientes respectivamente, mientras que los equipos serán colocados en el mismo orden de la tabla sobre su respectiva sub-corriente asociada a una fila.

Tabla 4.5. Distribución final de equipos en sub-corrientes de proceso calientes.

\begin{tabular}{|c|c|c|c|c|c|c|}
\hline $\begin{array}{c}\text { Corriente } \\
i\end{array}$ & Etapa 1 & Etapa 2 & Etapa 3 & Etapa 4 & Etapa 5 & Etapa 6 \\
\hline $\mathrm{H} 2$ & & 2 & 6 & 9 & 10 & \\
\hline $\mathrm{H} 2$ & & 3 & $(7$ & & & \\
\hline $\mathrm{H} 1$ & & & 4 & 8 & & \\
\hline $\mathrm{H} 1$ & & & $(5$ & & & \\
\hline
\end{tabular}

De forma análoga se puede realizar el mismo procedimiento para los intercambios de calor de las corrientes frías. En la Tabla 4.6 se proporciona el ordenamiento inicial de los equipos para las corrientes frías, guardando el orden de equipos otorgado en la distribución de cargas térmicas de la Tabla 4.3. El hecho de que se haya elegido como criterio de orden los intervalos de temperatura calientes, introduce la posibilidad de generar diferentes redes redundantes si se considera un ordenamiento diferente de la distribución de cargas térmicas, como pudiera ser con 
respecto a los intervalos de temperatura fríos, ofreciendo la oportunidad de generar redes de intercambio de calor con una configuración completamente diferente.

Tabla 4.6. Representación inicial por etapas de equipos en las corrientes frías.

\begin{tabular}{|c|c|c|c|c|c|c|}
\hline $\begin{array}{c}\text { Corriente } \\
j\end{array}$ & $\begin{array}{c}\text { Etapa } 1 \\
l 1=1\end{array}$ & $\begin{array}{c}\text { Etapa 2 } \\
l 1=2\end{array}$ & $\begin{array}{c}\text { Etapa } 3 \\
l 1=3\end{array}$ & $\begin{array}{c}\text { Etapa } 4 \\
l 1=4\end{array}$ & $\begin{array}{c}\text { Etapa } 5 \\
l 1=5\end{array}$ & $\begin{array}{c}\text { Etapa } 6 \\
l 1=6\end{array}$ \\
\hline $\mathrm{C} 1$ & & (2) & & & & \\
\hline $\mathrm{C} 2$ & & (3) & & & & \\
\hline $\mathrm{C} 1$ & & & (4) & & & \\
\hline $\mathrm{C} 2$ & & & (5) & & & \\
\hline $\mathrm{C} 1$ & & & (6) & & & \\
\hline $\mathrm{C} 1$ & & & & (7) & & \\
\hline $\mathrm{C} 1$ & & & & (8) & & \\
\hline $\mathrm{C} 1$ & & & & & & (9) \\
\hline $\mathrm{C} 1$ & & & & & (10) & \\
\hline
\end{tabular}

En la Figura 4.9 se muestra de forma aislada la comparación de la primera fila señalada con una flecha sólida, donde se ha procedido de la misma forma que en el caso de las corrientes calientes para colocar en serie a los 4 y 7 sobre las etapas 3 y 4 respectivamente. La comparación se realiza con la fila señalada con la flecha blanca a la izquierda que contiene al equipo 9. Este intercambiador de calor se encuentra en la etapa 6, la cual es posterior a la etapa 4 que corresponde al último equipo colocado en serie sobre la primera fila. El equipo 9 es colocado en serie sobre la fila marcada con la flecha sólida en la etapa 6 , por lo que la etapa 5 permanece sin ocuparse en la primera fila.

En la Figura 4.10 se presenta el caso donde la fila sobre la cual se está realizando el análisis marcada con la flecha sólida, presenta un equipo puesto en serie sobre la etapa 6 mientras que la fila comparada marcada con la flecha blanca, si bien pertenece a la misma corriente de proceso, no cumple el criterio de presentar un equipo en una etapa posterior al último equipo puesto en 
serie de la fila superior. Por esta razón, a pesar de que la etapa 5 no se encuentra ocupada en la primera fila, no se admite la colocación del equipo 10 en serie.

\begin{tabular}{|c|c|c|c|c|c|c|}
\hline $\begin{array}{c}\text { Corriente } \\
j\end{array}$ & $\begin{array}{c}\text { Etapa 1 } \\
l 1=1\end{array}$ & $\begin{array}{c}\text { Etapa 2 } \\
l l=2\end{array}$ & $\begin{array}{c}\text { Etapa 3 } \\
l l=3\end{array}$ & $\begin{array}{c}\text { Etapa 4 } \\
l l=4\end{array}$ & $\begin{array}{c}\text { Etapa 5 } \\
l 1=5\end{array}$ & $\begin{array}{c}\text { Etapa 6 } \\
l 1=6\end{array}$ \\
\hline $\mathrm{C} 1$ & & 2 & 4 & 7 & & 9 \\
\hline $\mathrm{C} 2$ & & 3 & & & & \\
\hline $\mathrm{C} 2$ & & & 5 & & & \\
\hline $\mathrm{C} 1$ & & & 6 & & & \\
\hline $\mathrm{C} 1$ & & & & 8 & & \\
\hline $\mathrm{C} 1$ & & & & & & \\
\hline $\mathrm{C} 1$ & & & & & & \\
\hline
\end{tabular}

Figura 4.9. El equipo 9 es colocado en serie dejando una entrada vacía.

Figura 4.10. El equipo 10 no cumple con los criterios para ser colocado en serie en la primera fila.

\begin{tabular}{|c|c|c|c|c|c|c|}
\hline $\begin{array}{c}\text { Corriente } \\
j\end{array}$ & $\begin{array}{c}\text { Etapa 1 } \\
l 1=1\end{array}$ & $\begin{array}{c}\text { Etapa 2 } \\
l 1=2\end{array}$ & $\begin{array}{c}\text { Etapa 3 } \\
l 1=3\end{array}$ & $\begin{array}{c}\text { Etapa 4 } \\
l 1=4\end{array}$ & $\begin{array}{c}\text { Etapa 5 } \\
l 1=5\end{array}$ & $\begin{array}{c}\text { Etapa 6 } \\
l 1=6\end{array}$ \\
\hline $\mathrm{C} 1$ & & 2 & 4 & 7 & & \\
\hline $\mathrm{C} 2$ & & 3 & & & \\
\hline $\mathrm{C} 2$ & & & 5 & & \\
\hline $\mathrm{C} 1$ & & & 6 & & \\
\hline $\mathrm{C} 1$ & & & & 8 & \\
\hline $\mathrm{C} 1$ & & & & & \\
\hline
\end{tabular}

En la Tabla 4.7 se definen finalmente los equipos disponibles sobre las filas que no fueron eliminadas para las corrientes frías, se puede distinguir que en la corriente de proceso $\mathrm{C} 1$ son necesarias dos sub-corrientes, mientras que la corriente de proceso $\mathrm{C} 2$ sólo está presente en una fila, esto significa que para la corriente $\mathrm{C} 2$ no necesita dividirse. 
Tabla 4.7. Distribución final de equipos en sub-corrientes de proceso frías.

\begin{tabular}{|c|c|c|c|c|c|c|}
\hline $\begin{array}{c}\text { Corriente } \\
j\end{array}$ & $\begin{array}{c}\text { Etapa 1 } \\
l l=1\end{array}$ & $\begin{array}{c}\text { Etapa 2 } \\
l 1=2\end{array}$ & $\begin{array}{c}\text { Etapa 3 } \\
l l=3\end{array}$ & $\begin{array}{c}\text { Etapa 4 } \\
l l=4\end{array}$ & $\begin{array}{c}\text { Etapa 5 } \\
l 1=5\end{array}$ & $\begin{array}{c}\text { Etapa 6 } \\
l l=6\end{array}$ \\
\hline $\mathrm{C} 1$ & & 2 & 4 & 7 & & 9 \\
\hline $\mathrm{C} 2$ & & 3 & 5 & & & \\
\hline $\mathrm{C} 1$ & & & 6 & 8 & 10 & \\
\hline
\end{tabular}

El uso de etapas es necesario para definir la posición de los equipos sobre las corrientes o subcorrientes de proceso concluyendo en la Tabla 4.5 para las corrientes calientes y la Tabla 4.7 para las corrientes frías. Por otro lado, el orden de los equipos se había definido desde la Tabla 4.2 siendo el primer intercambiador de calor el primer elemento de la tabla y así sucesivamente. Por medio de estas suposiciones es posible construir una representación sobre un diagrama de malla de la siguiente forma. El intercambiador de calor 1 se encuentra señalado en la Tabla 4.2 como un servicio de calentamiento extremo sobre la corriente C2. El segundo intercambiador de calor, de acuerdo con la Tabla 4.5 debe suministrar calor desde la primera sub-corriente de H2 y de acuerdo con la Tabla 4.7 debe recibir calor en la primera sub-corriente de C1, el intercambiador de calor 3 por su parte debe suministrar calor desde la segunda sub-corriente de $\mathrm{H} 2$ hacia la corriente de proceso C2sin divisiones. De esta manera es posible definir uno a uno la posición de cada intercambiador de calor sobre las corrientes o sub-corrientes de proceso hasta determinar una estructura de red base redundante como se detalla en la Figura 4.11.

La red redundante de la Figura 4.11 tiene la característica de distribuir los equipos disponibles a lo largo de diferentes sub-corrientes o corrientes de proceso del diagrama de malla, permitiendo definir un dominio factible amplio e incrementando la posibilidad de contener diversidad de óptimos locales, lo que significa una mayor probabilidad de revelar redes de intercambio de calor óptimas con CTA competitivo.

Uno de los aspectos más destacables de los diseños de red redundantes es que permiten colocar varios intercambiadores de calor en serie sobre una misma sub-corriente de proceso, estas estructuras no suelen incluirse en las metodologías de síntesis clásicas que utilizan el concepto de superestructuras (Yee y Grossmann, 1990 y Floudas y Ciric, 1989). Esto pudiera ser indicativo 
de que existe la posibilidad de evaluar soluciones mediante la optimización de topologías redundantes que han sido poco estudiadas.

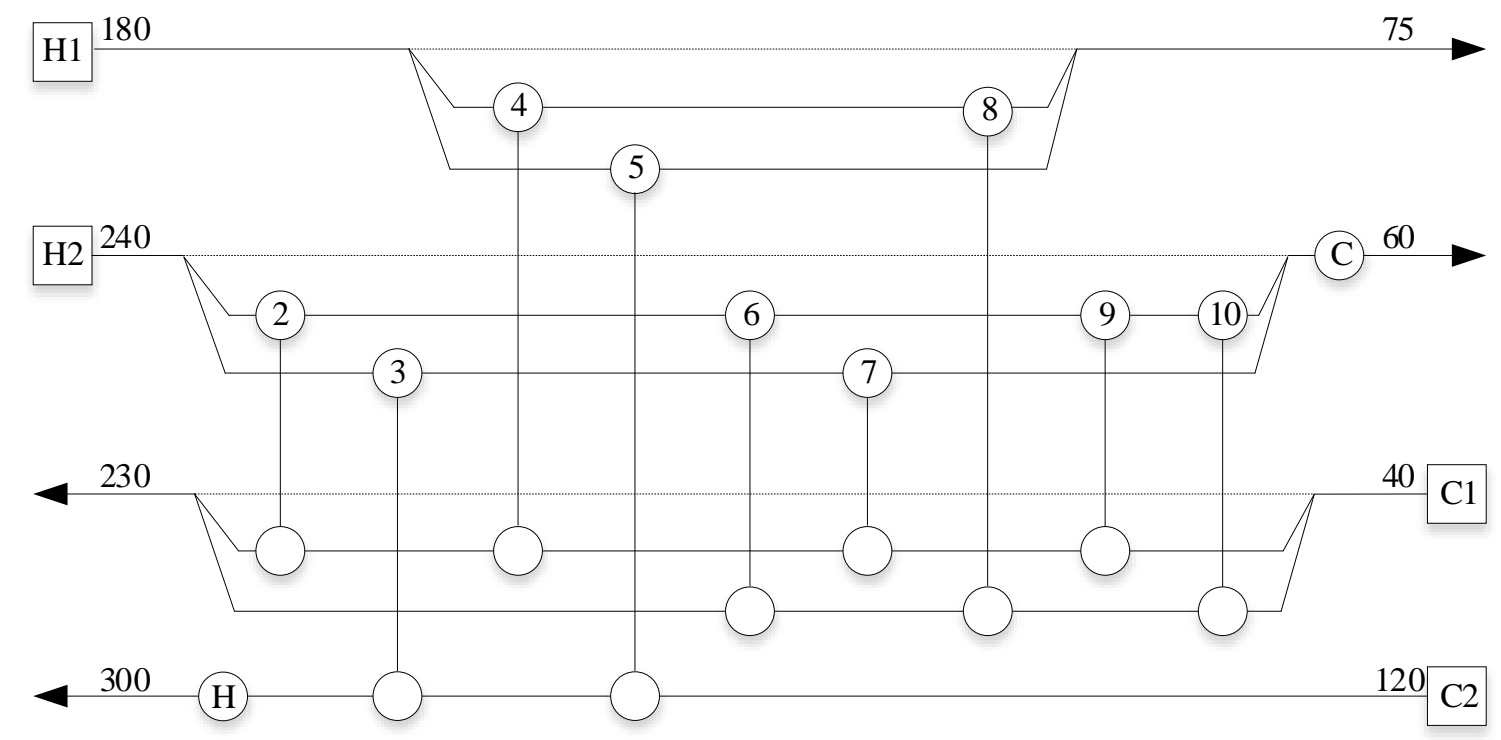

Figura 4.11. Diagrama de malla la topología redundante 1 para el ejemplo ilustrativo 4.1.

Es posible plantear formalmente un algoritmo que describa de forma sintética los pasos que deben seguirse para definir las sub-corrientes necesarias sobre las cuales los equipos deben ser posicionados, en otras palabras, que permita generar las Tablas 4.5 y 4.7 automáticamente. A continuación, se propone un algoritmo en forma de pseudocódigo con este propósito, para determinar la posición de equipos sobre corrientes o en caso de ser necesario sub-corrientes de proceso partiendo únicamente de la distribución de cargas térmicas inicial de la Tabla 4.3.

\section{PSEUDOCÓDIGO PARA LA GENERACIÓN DE LA TOPOLOGÍA REDUNDANTE 1}

1: Para cada fila $m$ que corresponde a intercambio de calor entre corrientes de proceso

2: $\quad$ Para cada fila $n$ que corresponde a intercambio de calor entre corrientes de proceso

3: $\quad$ Si $(n>m)$ entonces

4: $\quad$ Si $(i(n)=j(m)) \mathbf{y}(l(n)>l(m))$ entonces

5: $\quad$ El equipo $n$ se colca en serie sobre la misma sub-corriente de proceso caliente del equipo $m$

6: $\quad$ Se elimina la n-ésima sub-corriente de proceso caliente

7: $\quad l(m)=l(n)$ 


$\begin{array}{ll}\text { 8: } & \text { Fin Si } \\ \text { 9: } & \mathbf{S i}(j(n)=j(m)) \mathbf{y}(l 1(n)>l 1(m)) \text { entonces } \\ \text { 10: } & \text { El equipo } n \text { se coloca en serie sobre la misma sub-corriente de } \\ & \text { proceso fría del equipo } m \\ \text { 11: } & \text { Se elimina la } m \text {-ésima sub-corriente de proceso fría } \\ \text { 12: } & l 1(m)=l 1(n)\end{array}$

13: $\quad$ Fin Si

\section{4: $\quad$ Fin Si}

\section{5: $\quad$ Fin Para}

\section{6: Fin Para}

\section{Topología redundante 2}

Cada elemento de la tabla de distribución de cargas térmicas se ha considerado como un equipo de intercambio de calor en la topología redundante, sin embargo, es de suponer que en problemas que involucren un número elevado de corrientes de proceso los apareamientos indicados por la solución del modelo de transporte incrementen considerablemente. Lo anterior se traduce a topologías redundantes muy robustas con numerosos intercambiadores de calor y divisiones de corrientes. Entre más elementos presente una red de intercambio de calor redundante es necesario destinar un mayor tiempo de cómputo al proceso de optimización con el fin de asegurar que se han evaluado un número considerable soluciones óptimas que reduzcan la probabilidad de omitir soluciones de calidad.

Es posible redefinir la distribución de cargas térmicas para disminuir el número de intercambios de calor que conforman la Tabla 4.2 permitiendo generar una estructura de red redundante con un menor número de equipos en comparación con la planteada en la Figura 4.11. Esta propuesta reduce el espacio de solución, y también genera un dominio factible diferente, lo que significa que cada diseño base pudiera contener soluciones óptimas diferentes. Por esta razón los diseños de red iniciales generados mediante los esquemas propuestos deben considerarse como alternativas para la generación de redes redundantes de intercambio de calor, que dan la opción al diseñador de elegir aquella que se ajuste mejor a sus necesidades. 
Se plantea disminuir en número las filas de la Tabla 4.2 lo que implica una menor cantidad disponible de intercambiadores de calor. El procedimiento consiste en integrar las cargas térmicas donde una misma corriente de proceso caliente interactúa con una misma corriente de proceso fría sobre un mismo intervalo de temperatura o en intervalos de temperatura contiguos.

En la Figura 4.12 se muestra del lado izquierdo la distribución de cargas térmicas de la Tabla 4.2. Dentro del recuadro se remarcan dos cargas térmicas donde la corriente de proceso H1 intercambia calor con la corriente $\mathrm{C} 1$. Como los intervalos de temperatura $l$ y $l 1$ de ambos apareamientos presentan el mismo número o números consecutivos, es posible integrarlos y representarlos como una sola carga térmica. Del lado derecho se ilustra la manera en la que los dos apareamientos se ponen a disposición sobre una nueva tabla de distribución de cargas térmicas, para esto se definen nuevos intervalos de temperatura que guardan la información del primer y el último intervalo de temperatura $\left(l_{\min }-l_{\max }\right)$ donde las corrientes intercambian calor.

\begin{tabular}{|c|c|c|c|c|c|c|c|c|c|}
\hline$i$ & $l$ & $j$ & $l 1$ & $\begin{array}{c}q \\
(\mathrm{~kW})\end{array}$ & $i$ & $l_{\text {min }}-l_{\text {max }}$ & $j$ & $l 1_{\text {min }}-l 1_{\text {max }}$ & $\begin{array}{c}q \\
(\mathrm{~kW})\end{array}$ \\
\hline qhu & - & 2 & - & 2300 & qhu & - & 2 & - & 2300 \\
\hline 1 & 3 & 1 & 3 & 500 & \multirow{10}{*}{1} & \multirow{10}{*}{$3-4$} & \multirow{10}{*}{1} & \multirow{10}{*}{$3-4$} & \multirow{10}{*}{2150} \\
\hline 1 & 4 & 1 & 4 & 1650 & & & & & \\
\hline 1 & 3 & 2 & 3 & 1000 & & & & & \\
\hline 2 & 2 & 1 & 2 & 2100 & & & & & \\
\hline 2 & 3 & 1 & 3 & 1250 & & & & & \\
\hline 2 & 3 & 1 & 4 & 275 & & & & & \\
\hline 2 & 4 & 1 & 6 & 350 & & & & & \\
\hline 2 & 5 & 1 & 5 & 525 & & & & & \\
\hline 2 & 2 & 2 & 2 & 300 & & & & & \\
\hline 2 & - & qcu & - & 2400 & & & & & \\
\hline
\end{tabular}

Figura 4.12. Integración de dos apareamientos para definir una distribución de cargas térmicas reducida.

En la Figura 4.13 se resalta mediante un recuadro el apareamiento que indica que la corriente de proceso $\mathrm{H} 1$ debe intercambiar calor con la corriente $\mathrm{C} 2$, sin embargo, el apareamiento que le sigue no corresponde a la misma corriente de proceso fría por lo que no se puede integrar, manteniéndose con sus mismos valores como se muestra en el lado derecho de la figura. 


\begin{tabular}{|c|c|c|c|c|c|c|c|c|c|}
\hline$i$ & $l$ & $j$ & $l 1$ & $\begin{array}{c}q \\
(\mathrm{~kW})\end{array}$ & $i$ & $l_{\min }-l_{\max }$ & $j$ & $l 1_{\min }-l 1_{\max }$ & $\begin{array}{c}q \\
(\mathrm{~kW})\end{array}$ \\
\hline qhu & - & 2 & - & 2300 & qhu & - & 2 & - & 2300 \\
\hline 1 & 3 & 1 & 3 & 500 & \multirow{2}{*}{1} & \multirow{2}{*}{$3-4$} & \multirow{2}{*}{1} & \multirow{2}{*}{$3-4$} & \multirow{2}{*}{2150} \\
\hline 1 & 4 & 1 & 4 & 1650 & & & & & \\
\hline 1 & 3 & 2 & 3 & 1000 & 1 & $3-3$ & 2 & $3-3$ & 1000 \\
\hline 2 & 2 & 1 & 2 & 2100 & & & & & \\
\hline 2 & 3 & 1 & 3 & 1250 & & & & & \\
\hline 2 & 3 & 1 & 4 & 275 & & & & & \\
\hline 2 & 4 & 1 & 6 & 350 & & & & & \\
\hline 2 & 5 & 1 & 5 & 525 & & & & & \\
\hline 2 & 2 & 2 & 2 & 300 & & & & & \\
\hline 2 & - & qcu & - & 2400 & & & & & \\
\hline
\end{tabular}

Figura 4.13. Apareamiento que permanece igual en la distribución de cargas térmicas reducida.

En la Figura 4.14 se ilustra el caso donde tres apareamientos corresponden a la corriente caliente $\mathrm{H} 2$ que intercambia calor con la corriente $\mathrm{C} 1$, los intercambios de calor se llevan a cabo sobre los mismos intervalos de temperatura o sobre un intervalo de temperatura con un número inmediato superior, por lo que se cumplen los criterios que permiten integrarlos en un solo apareamiento.

\begin{tabular}{|c|c|c|c|c|c|c|c|c|c|}
\hline$i$ & $l$ & $j$ & 11 & $\begin{array}{c}q \\
(\mathrm{~kW})\end{array}$ & $i$ & $l_{\min }-l_{\max }$ & $j$ & $l 1_{\min }-l 1_{\max }$ & $\begin{array}{c}q \\
(\mathrm{~kW})\end{array}$ \\
\hline qhu & - & 2 & - & 2300 & qhu & - & 2 & - & 2300 \\
\hline 1 & 3 & 1 & 3 & 500 & 1 & 34 & 1 & 34 & 2150 \\
\hline 1 & 4 & 1 & 4 & 1650 & 1 & $5-4$ & 1 & $5-4$ & 2150 \\
\hline 1 & 3 & 2 & 3 & 1000 & 1 & $3-3$ & 2 & $3-3$ & 1000 \\
\hline 2 & 2 & 1 & 2 & 2100 & & & & & \\
\hline 2 & 3 & 1 & 3 & 1250 & 2 & $2-3$ & 1 & $2-4$ & 3625 \\
\hline 2 & 3 & 1 & 4 & 275 & & & & & \\
\hline 2 & 4 & 1 & 6 & 350 & 2 & 4 & 1 & 6 & 350 \\
\hline 2 & 5 & 1 & 5 & 525 & 2 & 5 & 1 & 5 & 525 \\
\hline 2 & 2 & 2 & 2 & 300 & 2 & 2 & 2 & 2 & 300 \\
\hline 2 & - & qcu & - & 2400 & 2 & - & qcu & - & 2400 \\
\hline
\end{tabular}

Figura 4.14. Integración de tres apareamientos para definir una distribución de cargas térmicas reducida.

Para el caso del siguiente intercambio de calor $q_{2,4,1,6}$ a pesar de que también presentaba un apareamiento entre la corriente caliente $\mathrm{H} 2$ y la corriente fría $\mathrm{C} 1$, no corresponde a un intervalo 
de temperatura consecutivo por parte de la corriente fría por lo que no fue integrado con los tres apareamientos señalados en el recuadro de la Figura 4.14. Por otro lado, los intercambios de calor asociados a los elementos $q_{2,4,1,6}$ y $q_{2,4,1,5}$ relacionan a las mismas corrientes de proceso entre sí, sin embargo, el intervalo de temperatura frío del apareamiento que se encuentra debajo en la tabla no es un número inmediato superior al elemento anterior de la tabla, por lo que tampoco pueden ser integrados. Con los casos anteriores descritos, se concluye la construcción de la nueva distribución de cargas térmicas sin integrar más apareamientos como se muestra en el lado derecho de la Figura 4.14, la cual se reescribe en la Tabla 4.8.

Tabla 4.8. Distribución de cargas térmicas reducida

\begin{tabular}{ccccc}
\hline$i$ & $l_{\min }-l_{\max }$ & $j$ & $l 1_{\min }-l l_{\max }$ & $\begin{array}{c}q \\
(\mathrm{~kW})\end{array}$ \\
\hline $\mathrm{q}_{\mathrm{hu}}$ & - & 2 & - & 2300 \\
\hline 1 & $3-4$ & 1 & $3-4$ & 2150 \\
1 & $3-3$ & 2 & $3-3$ & 1000 \\
2 & $2-3$ & 1 & $2-4$ & 3625 \\
2 & $4-4$ & 1 & $6-6$ & 350 \\
2 & $5-5$ & 1 & $5-5$ & 525 \\
2 & $2-2$ & 2 & $2-2$ & 300 \\
\hline 2 & - & $\mathrm{q}_{\mathrm{cu}}$ & - & 2400 \\
\hline
\end{tabular}

La Tabla 4.8 se reordena la nueva distribución de cargas térmicas para asociar un orden de los intercambiadores de calor con respecto a los intervalos de temperatura calientes $\left(l_{\text {min }}\right)$ marcados en negritas en la Tabla 4.9.

Tabla 4.9. Distribución de cargas térmicas reducida, ordenada con respecto a los intervalos iniciales de temperatura calientes.

\begin{tabular}{cccccc}
\hline & & $\Delta$ & & & $q$ \\
$\mathrm{IC}$ & $i$ & $\boldsymbol{l}_{\min }-l_{\max }$ & $j$ & $l l_{\min }-l l_{\max }$ & $(\mathrm{kW})$ \\
\hline 1 & $\mathrm{qhu}$ & - & 2 & - & 2300 \\
2 & 2 & $\mathbf{2}-2$ & 2 & $2-2$ & 300 \\
3 & 2 & $\mathbf{2}-3$ & 1 & $2-4$ & 3625 \\
4 & 1 & $\mathbf{3}-3$ & 2 & $3-3$ & 1000 \\
5 & 1 & $\mathbf{3}-4$ & 1 & $3-4$ & 2150 \\
6 & 2 & $\mathbf{4}-4$ & 1 & $6-6$ & 350 \\
7 & 2 & $\mathbf{5}-5$ & 1 & $5-5$ & 525 \\
\hline 8 & 2 & - & $\mathrm{qcu}$ & - & 2400 \\
\hline
\end{tabular}


De igual manera que para la Topología redundante 1 se busca definir una configuración sobre la cual sea posible distribuir los intercambiadores de calor en corrientes o sub-corrientes de proceso según sea el caso. En la Tabla 4.10 se plantea, para las corrientes de proceso calientes, una representación donde los equipos pueden abarcar más de una etapa dependiendo de los valores de los intervalos de temperatura $l_{\min } \mathrm{y} l_{\max }$. $\mathrm{Al}$ análisis por filas para colocar equipos en serie procede de forma similar que para la Topología redundante 1.

Tabla 4.10. Representación inicial por etapas de equipos en las corrientes calientes con apareamientos integrados.

\begin{tabular}{|c|c|c|c|c|c|c|}
\hline $\begin{array}{c}\text { Corriente } \\
i\end{array}$ & $\begin{array}{c}\text { Etapa } 1 \\
l=1\end{array}$ & $\begin{array}{c}\text { Etapa } 2 \\
l=2\end{array}$ & $\begin{array}{c}\text { Etapa } 3 \\
l=3\end{array}$ & $\begin{array}{c}\text { Etapa } 4 \\
l=4\end{array}$ & $\begin{array}{c}\text { Etapa } 5 \\
l=5\end{array}$ & $\begin{array}{c}\text { Etapa } 6 \\
l=6\end{array}$ \\
\hline $\mathrm{H} 2$ & & $\vdash 2 \dashv$ & & & & \\
\hline $\mathrm{H} 2$ & & & $3-1$ & & & \\
\hline H1 & & & $\vdash 4 \dashv$ & & & \\
\hline H1 & & & & $5-1$ & & \\
\hline $\mathrm{H} 2$ & & & & $\vdash 6 \dashv$ & & \\
\hline $\mathrm{H} 2$ & & & & & $\vdash 7 \dashv$ & \\
\hline
\end{tabular}

En la Figura 4.15 se muestra le caso donde se realiza la comparación de la primera y segunda fila. El criterio para colocar equipos en serie consiste en que la fila inferior marcada con una flecha blanca debe presentar un equipo que corresponda a la misma corriente de proceso y su intervalo de temperatura inicial $\left(l_{\min }\right)$ se debe encontrar en una etapa mayor o igual que el intervalo de temperatura final $\left(l_{\max }\right)$ del último equipo puesto en serie de la fila superior marcada con la flecha sólida. De igual manera la fila que no presenta algún intercambiador de calor debe ser eliminada.

\begin{tabular}{|c|c|c|c|}
\hline $\begin{array}{c}\text { Corriente } \\
i\end{array}$ & $\begin{array}{c}\text { Etapa 1 } \\
l=1\end{array}$ & $\begin{array}{c}\text { Etapa 2 } \\
l=2\end{array}$ & $\begin{array}{c}\text { Etapa } 3 \\
l=3\end{array}$ \\
\hline $\mathrm{H} 2$ & & $\vdash 2-\vdash$ & $3-1<$ \\
\hline $\mathrm{H} 2$ & & & $3-1$ \\
\hline
\end{tabular}

Figura 4.15. Equipos colocados en serie utilizando un esquema reducido de apareamientos. 
Mediante esta propuesta es posible colocar equipos en serie y proceder a la comparación de cada fila como se hizo para la Topología redundante 1, se obtiene una distribución final de equipos sobre las corrientes de proceso calientes, como se muestra en la Tabla 4.11.

Tabla 4.11. Distribución final de equipos en sub-corrientes de proceso calientes, con apareamientos integrados.

\begin{tabular}{|c|c|c|c|c|c|c|}
\hline $\begin{array}{c}\text { Corriente } \\
i\end{array}$ & $\begin{array}{c}\text { Etapa 1 } \\
l=1\end{array}$ & $\begin{array}{c}\text { Etapa 2 } \\
l=2\end{array}$ & $\begin{array}{c}\text { Etapa 3 } \\
l=3\end{array}$ & $\begin{array}{c}\text { Etapa 4 } \\
l=4\end{array}$ & $\begin{array}{c}\text { Etapa 5 } \\
l=5\end{array}$ & $\begin{array}{c}\text { Etapa 6 } \\
l=6\end{array}$ \\
\hline $\mathrm{H} 2$ & & $-2-1-3 \dashv$ & $\vdash 6 \dashv$ & $\vdash 7-1$ & \\
\hline $\mathrm{H} 1$ & & & & $\vdash-1-5-1$ & \\
\hline
\end{tabular}

Para las corrientes de proceso frías se realiza el mismo procedimiento partiendo de la distribución inicial planteada en la Tabla 4.12. Y mediante el debido análisis comparativo por filas es posible concluir en la Tabla 4.13 que indica las sub-corrientes de proceso frías y el acomodo de los equipos sobre estas.

Tabla 4.12. Representación inicial por etapas de equipos en las corrientes frías con apareamientos integrados.

\begin{tabular}{|c|c|c|c|c|c|c|}
\hline $\begin{array}{c}\text { Corriente } \\
j\end{array}$ & $\begin{array}{c}\text { Etapa } 1 \\
l 1=1\end{array}$ & $\begin{array}{c}\text { Etapa } 2 \\
l 1=2\end{array}$ & $\begin{array}{c}\text { Etapa } 3 \\
l 1=3\end{array}$ & $\begin{array}{c}\text { Etapa } 4 \\
l 1=4\end{array}$ & $\begin{array}{c}\text { Etapa } 5 \\
l 1=5\end{array}$ & $\begin{array}{c}\text { Etapa } 6 \\
l 1=6\end{array}$ \\
\hline $\mathrm{C} 2$ & & $\vdash 2 \dashv$ & & & & \\
\hline $\mathrm{C} 1$ & & & $-3-$ & $H$ & & \\
\hline $\mathrm{C} 2$ & & & $\vdash 4 \dashv$ & & & \\
\hline $\mathrm{C} 1$ & & & $\vdash$ & $;-1$ & & \\
\hline $\mathrm{C} 1$ & & & & & & $\vdash 6-1$ \\
\hline $\mathrm{C} 1$ & & & & & $\vdash 7 \dashv$ & \\
\hline
\end{tabular}

Los equipos pueden ser posicionados sobre un diagrama de malla con el orden otorgado por la Tabla 4.9 y distribuyéndolos sobre las corrientes y sub-corrientes de proceso como se indica en la Tabla 4.11 para las corrientes calientes y la Tabla 4.13 para las corrientes frías. El diseño de 
red es mostrado en la Figura 4.16 el cual presenta una reducción de tres equipos con respecto a la Topología redundante 1 descrita en la Figura 4.11 la cual no integra apareamientos.

Tabla 4.13. Distribución final de equipos en sub-corrientes de proceso calientes, con apareamientos integrados.

\begin{tabular}{|c|c|c|c|c|c|c|}
\hline $\begin{array}{c}\text { Corriente } \\
j\end{array}$ & $\begin{array}{c}\text { Etapa 1 } \\
l 1=1\end{array}$ & $\begin{array}{c}\text { Etapa 2 } \\
l 1=2\end{array}$ & $\begin{array}{c}\text { Etapa 3 } \\
l 1=3\end{array}$ & $\begin{array}{c}\text { Etapa 4 } \\
l 1=4\end{array}$ & $\begin{array}{c}\text { Etapa 5 } \\
l 1=5\end{array}$ & $\begin{array}{c}\text { Etapa 6 } \\
l 1=6\end{array}$ \\
\hline $\mathrm{C} 2$ & & $\vdash 2 \dashv$ & $\vdash 4 \dashv$ & & & \\
\hline $\mathrm{C} 1$ & & & & & & $\vdash 6 \dashv$ \\
\hline $\mathrm{C} 1$ & & & $\vdash 5 \dashv$ & $\vdash 7-1$ & \\
\hline
\end{tabular}

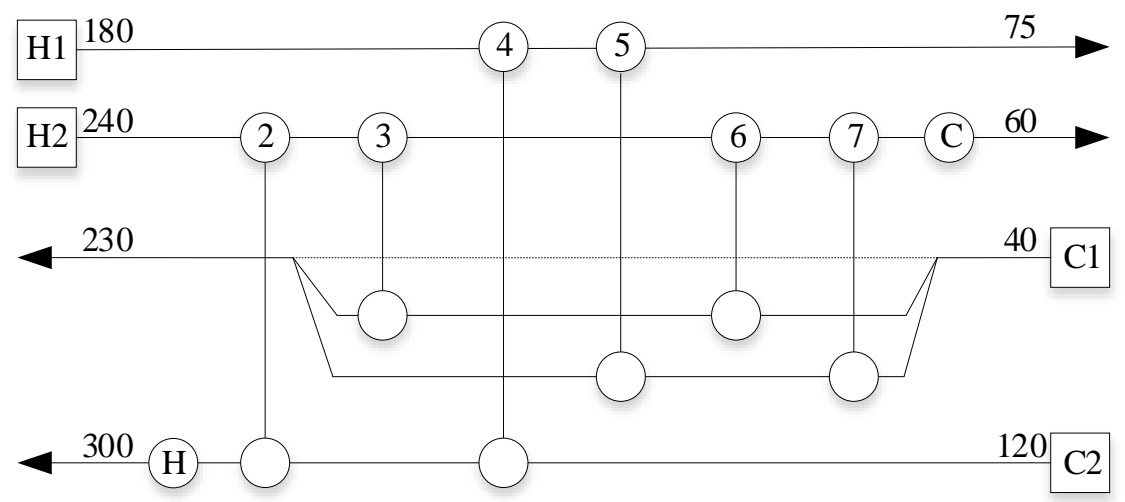

Figura 4.16. Diagrama de malla de la red redundante integración de apareamientos para el ejemplo ilustrativo 4.1 .

De igual manera que para la Topología redundante 1, es posible plantear un algoritmo en forma de pseudocódigo que genere de forma automática la distribución de equipos sobre las corrientes y sub-corrientes de proceso que comprenden a las Tablas 4.11 y 4.13 a partir de los elementos de la distribución de cargas térmicas con apareamientos reducidos de la Tabla 4.9.

\section{PSEUDOCÓDIGO PARA LA GENERACIÓN DE LAS TOPOLOGÍAS REDUNDANTES 2 Y 3}

1: Para cada fila $m$ que corresponde a intercambio de calor entre corrientes de proceso

2: $\quad$ Para cada fila $n$ que corresponde a intercambio de calor entre corrientes de proceso

3: $\quad$ Si $(m>n)$ entonces

4: $\quad$ Si $(i(n)=i(m)) \mathbf{y}\left(l_{\max }(m) \leq l_{\min }(n)\right) \mathbf{y}\left(l_{\max }(n)>l_{\text {min }}(m)\right)$ entonces 
5: $\quad$ El equipo $n$ se colca en serie sobre la misma sub-corriente de proceso caliente del equipo $m$

6: Se elimina la $n$-ésima sub-corriente de proceso caliente

7:

$l_{\max }(m)=l_{\max }(n)$

8:

Fin Si

9:

Si $(j(n)=j(m)) \mathbf{y}\left(l 1_{\text {max }}(m) \leq l 1_{\text {min }}(n)\right) \mathbf{y}\left(l_{\text {max }}(n)>l_{\text {min }}(m)\right)$ entonces

10:

El equipo $n$ se colca en serie sobre la misma sub-corriente de proceso fría del equipo $m$

11:

Se elimina la $n$-ésima sub-corriente de proceso fría

12:

$l 1_{\max }(m)=l 1_{\max }(n)$

13:

Fin Si

14:

\section{Fin Si}

\section{5: Fin Para}

\section{6: Fin Para}

\section{Topología redundante 3}

En esta sección se formula una última propuesta para la generación de una red redundante con el menor número de equipos posibles. Cada esquema presenta diferentes características y por lo tanto es posible determinar diferentes óptimos locales con cada uno. Esta variación es planteada bajo la premisa de poder realizar diseños base sencillos para una rápida evaluación de soluciones óptimas locales en un número modesto de iteraciones, lo que significa un ahorro en términos de recursos computacionales.

El planteamiento consiste en eliminar por completo la posibilidad de reapareamientos, esto quiere decir que cada corriente de proceso caliente solo puede intercambiar calor a lo más una vez con cada corriente de proceso fría. Al igual que para la Topología redundante 2 se integran los apareamientos contiguos en la distribución de cargas térmicas, pero en este caso el único criterio a considerar es que la misma corriente de proceso caliente intercambie calor con la misma corriente fría de proceso sin importar sobre que intervalos de temperatura se encuentren. 
Bajo las afirmaciones realizadas es posible reducir considerablemente el número de apareamientos de la distribución de cargas térmicas significando una menor disponibilidad de intercambios de calor entre corrientes de proceso. En la Figura 4.17 se muestra la distribución de cargas térmicas original, el primer recuadro señala a la corriente caliente $\mathrm{H} 2$ suministrando calor la corriente $\mathrm{C} 1$ por una serie de intervalos, mientras que el segundo recuadro indica que la corriente $\mathrm{H} 2$ intercambia calor con la corriente $\mathrm{C} 1$. Sin importar los intervalos de temperatura que estos apareamientos tengan asociados se representan por un único intercambio de calor como se muestra al lado derecho de la figura, siempre guardando registro del primer y último intervalo de temperatura donde se realiza la integración.

\begin{tabular}{|c|c|c|c|c|c|c|c|c|c|}
\hline$i$ & $l$ & $j$ & $l 1$ & $\begin{array}{c}q \\
(\mathrm{~kW})\end{array}$ & $i$ & $l_{\min }-l_{\max }$ & $j$ & $l 1_{\min }-l 1_{\max }$ & $\begin{array}{c}q \\
(\mathrm{~kW})\end{array}$ \\
\hline qhu & - & 2 & - & 2300 & qhu & - & 2 & - & 2300 \\
\hline 1 & 3 & 1 & 3 & 500 & \multirow{2}{*}{1} & \multirow{2}{*}{$3-4$} & \multirow{2}{*}{1} & \multirow{2}{*}{$3-4$} & \multirow{2}{*}{2150} \\
\hline 1 & 4 & 1 & 4 & 1650 & & & & & \\
\hline 1 & 3 & 2 & 3 & 1000 & \multirow[t]{3}{*}{1} & \multirow[t]{3}{*}{$3-3$} & \multirow[t]{3}{*}{2} & \multirow[t]{3}{*}{$3-3$} & \multirow[t]{3}{*}{1000} \\
\hline 2 & 2 & 1 & 2 & 2100 & & & & & \\
\hline 2 & 3 & 1 & 3 & 1250 & & & & & \\
\hline 2 & 3 & 1 & 4 & 275 & \multirow[t]{3}{*}{2} & \multirow[t]{3}{*}{$2-5$} & \multirow[t]{3}{*}{1} & \multirow[t]{3}{*}{$2-6$} & \multirow[t]{3}{*}{4500} \\
\hline 2 & 4 & 1 & 6 & 350 & & & & & \\
\hline 2 & 5 & 1 & 5 & 525 & & & & & \\
\hline 2 & 2 & 2 & 2 & 300 & 2 & $2-2$ & 2 & $2-2$ & 300 \\
\hline 2 & - & qcu & - & 2400 & 2 & - & $\mathrm{q}_{\mathrm{cu}}$ & - & 2400 \\
\hline
\end{tabular}

Figura 4.17. Integración de tres apareamientos para definir una distribución de cargas térmicas sin reapareamientos.

Tabla 4.14. Distribución de cargas térmicas sin reapareamientos.

\begin{tabular}{ccccc}
\hline$i$ & $l_{\min }-l_{\max }$ & $j$ & $l l_{\min }-l l_{\max }$ & $\begin{array}{c}q \\
(\mathrm{~kW})\end{array}$ \\
\hline $\mathrm{qhu}$ & - & 2 & - & 2300 \\
\hline 1 & $3-4$ & 1 & $3-4$ & 2150 \\
1 & $3-3$ & 2 & $3-3$ & 1000 \\
2 & $2-5$ & 1 & $2-6$ & 4500 \\
2 & $2-2$ & 2 & $2-2$ & 300 \\
\hline 2 & - & $\mathrm{qcu}$ & - & 2400 \\
\hline
\end{tabular}


En la Tabla 4.14 se muestra la nueva distribución de cargas térmicas que reduce considerablemente los intercambios de calor entre diferentes corrientes de proceso. De los doce apareamientos determinados por la solución del modelo de transporte originalmente, únicamente se conservan seis con el planteamiento propuesto. En problemas que involucran un número considerable de corrientes de proceso, las variantes con reducciones de intercambios de calor pueden ser adecuadas para determinar soluciones en tiempos de cómputo razonables.

La Tabla 4.14 se reordena con respecto a los intervalos de temperatura calientes $\left(l_{\text {min }}\right)$ para asignar el orden de los intercambiadores de calor (IC) como se ilustra en la Tabla 4.15. Exactamente el mismo procedimiento o pseudocódigo desarrollado para la Topología redúndate 2 es usado para generar la distribución de equipos sobre las sub-corrientes de proceso partir de la Tabla 4.15. El diseño de red resultante se ilustra en la Figura 4.18.

Tabla 4.15. Distribución de cargas térmicas sin reapareamientos, ordenada

\begin{tabular}{|c|c|c|c|c|c|}
\hline$I C$ & $i$ & ${\overrightarrow{l_{\min }}}^{\mathbf{1}} l_{\max }$ & $j$ & $l 1_{\text {min }}-l 1_{\text {max }}$ & $\begin{array}{c}q \\
(\mathrm{~kW})\end{array}$ \\
\hline 1 & $\mathrm{q}_{\mathrm{hu}}$ & - & 2 & - & 2300 \\
\hline 2 & 2 & $2-2$ & 2 & $2-2$ & 300 \\
\hline 3 & 2 & $2-5$ & 1 & $2-6$ & 4500 \\
\hline 4 & 1 & $3-3$ & 2 & $3-3$ & 1000 \\
\hline 5 & 1 & $3-4$ & 1 & $3-4$ & 2150 \\
\hline 6 & 2 & - & $\mathrm{q}_{\mathrm{cu}}$ & - & 2400 \\
\hline
\end{tabular}

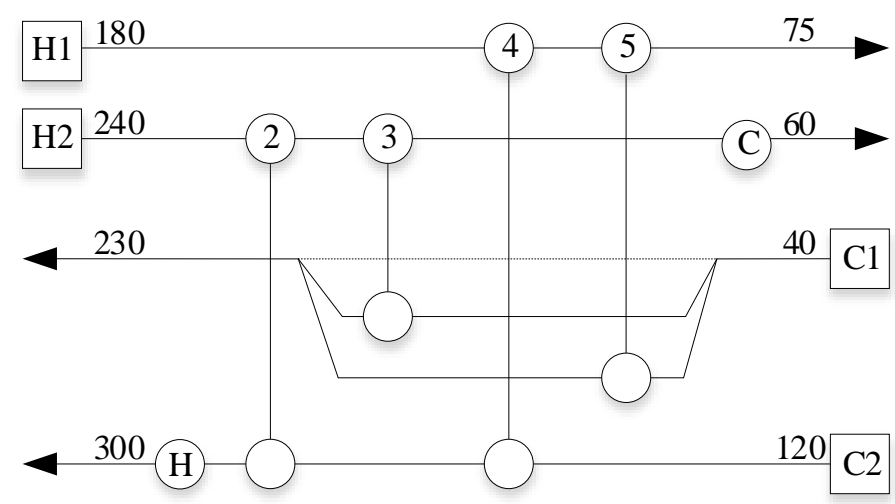

Figura 4.18. Diagrama de malla de la red redundante sin reapareamientos para el ejemplo ilustrativo 4.1 . 


\subsection{Variación del HRAT para generación de diferentes redes redundantes}

Las topologías redundantes generadas en la Sección 4.3 están basadas en intercambios de calor interpretados como apareamientos otorgados por la solución del problema de transporte para el uso mínimo de servicios auxiliares. El modelo de transporte (Cerdá y col., 1983) está diseñado sobre elementos conceptuales provenientes del método de punto de pliegue haciendo uso de intervalos de temperatura (Flower y Linnhoff, 1978). Mediante principios heurísticos la metodología de punto de pliegue permite definir el nivel de recuperación de energía por medio del parámetro HRAT. Este parámetro indica en la tabla de calor, la posición relativa entre

corrientes calientes y frías identificando el punto donde se encuentran más restringidas a recuperar calor.

Como se discutió en el Capítulo 2 el desplazamiento relativo de las corrientes debido a la variación del parámetro HRAT, define diferentes de intervalos de temperatura y en términos de la metodología de síntesis propuesta, significa la posibilidad de obtener diferentes rutas de intercambio de calor entre corrientes de proceso, abriendo la posibilidad de generar diferentes redes de intercambio de calor redundantes.

Se ha investigado la generación de topologías redundantes mediante la solución del modelo de transporte para un HRAT dado, y se han propuesto algunos esquemas que permiten reducir los apareamientos entre corrientes de proceso para generar estructuras de red con diferentes números de equipos. No obstante, es muy probable que algunos apareamientos otorgados por la solución del modelo de transporte generen diseños base que pudieran no contener soluciones atractivas en términos de costo, esto significa que la evaluación de un solo diseño base no es suficiente para asegurar que se dispone de una solución de calidad.

Debido a que la generación de redes redundantes se ha propuesto con el fin de formar parte de una metodología sistemática, la variación del parámetro HRAT en el modelo de transporte, introduce la posibilidad de obtener diferentes apareamientos entre corrientes de proceso traduciéndose en la oportunidad de generar múltiples diseños base, cada uno a su vez conteniendo diferentes óptimos locales, no obstante, un criterio pobre para la elección de este parámetro pudiera afectar de forma negativa los intercambios de calor disponibles para generar estructuras redundantes poco convenientes. 
El método de "Super-Targeting" (Linnhoff y Hindmarsh, 1983) provee una herramienta útil, capaz de determinar estimaciones del costo de una red de intercambio de calor sin proponer un diseño de red en específico, en función HRAT. Con esta técnica se puede estimar el valor del HRAT óptimo, permitiendo al usuario definir un conjunto de parámetros alrededor de este valor que presenten buenas estimaciones en su costo de acuerdo con el método de "Super-Targeting" y que permitan generar diversas topologías redundantes. Para más detalles de la tecnología de punto de pliegue y del método de "Super-Targeting" se pueden consultar diversos textos disponibles en la literatura como son, Shenoy (1995), Smith (2005) y Kemp (2011).

\subsection{Metodología secuencial para la síntesis de redes de intercambio de calor}

Con los elementos desarrollados, es posible definir una metodología secuencial para la síntesis de redes de intercambio de calor. El enfoque propuesto es de carácter iterativo y se considera secuencial puesto que consiste en descomponer el problema original en dos sub-problemas de generación y optimización de estructuras de red redundantes. La formulación desarrollada en este trabajo se plantea como una alternativa a las metodologías de síntesis clásicas, con la principal diferencia de que los múltiples diseños de red a optimizar presentan un dominio factible establecido que evitan que el espacio de solución crezca exponencialmente.

En la Figura 4.19 se ilustra la secuencia de la metodología de síntesis propuesta para determinar diseños óptimos mediante la generación y optimización de topologías redundantes, dicha formulación se apoya en la notación del Capítulo 3. El algoritmo se inicia con el método de preoptimización de "Super-Targeting" para determinar el valor HRAT* óptimo, el cual presenta el valor más bajo en su estimación de costo. El usuario debe proponer un intervalo de parámetros acotados por un $\mathrm{HRAT}^{\mathrm{L}}$ inicial y un $\mathrm{HRAT}^{\mathrm{U}}$ final que contengan al $\mathrm{HRAT}^{*}$ y que también presenten estimaciones de costos razonables para la generación de las múltiples topologías redundantes. Se asigna el costo de la mejor solución disponible en $\mathrm{CTA}^{*}=\infty$ y se inicializa la generación de estructuras en el valor definido $\operatorname{HRAT}^{\mathrm{L}}$, se propone un tamaño de paso $t$ que incrementa el valor del HRAT en cada iteración hasta llegar al $\mathrm{HRAT}^{\mathrm{U}}$ final. Se utiliza el valor para la iteración actual $\mathrm{HRAT}_{\mathrm{a}}$ para resolver el primer sub-problema de síntesis que consiste en 
generar una topología redundante, mediante una distribución de cargas térmicas obtenida de la solución del modelo de transporte (Cerdá y col., 1983).

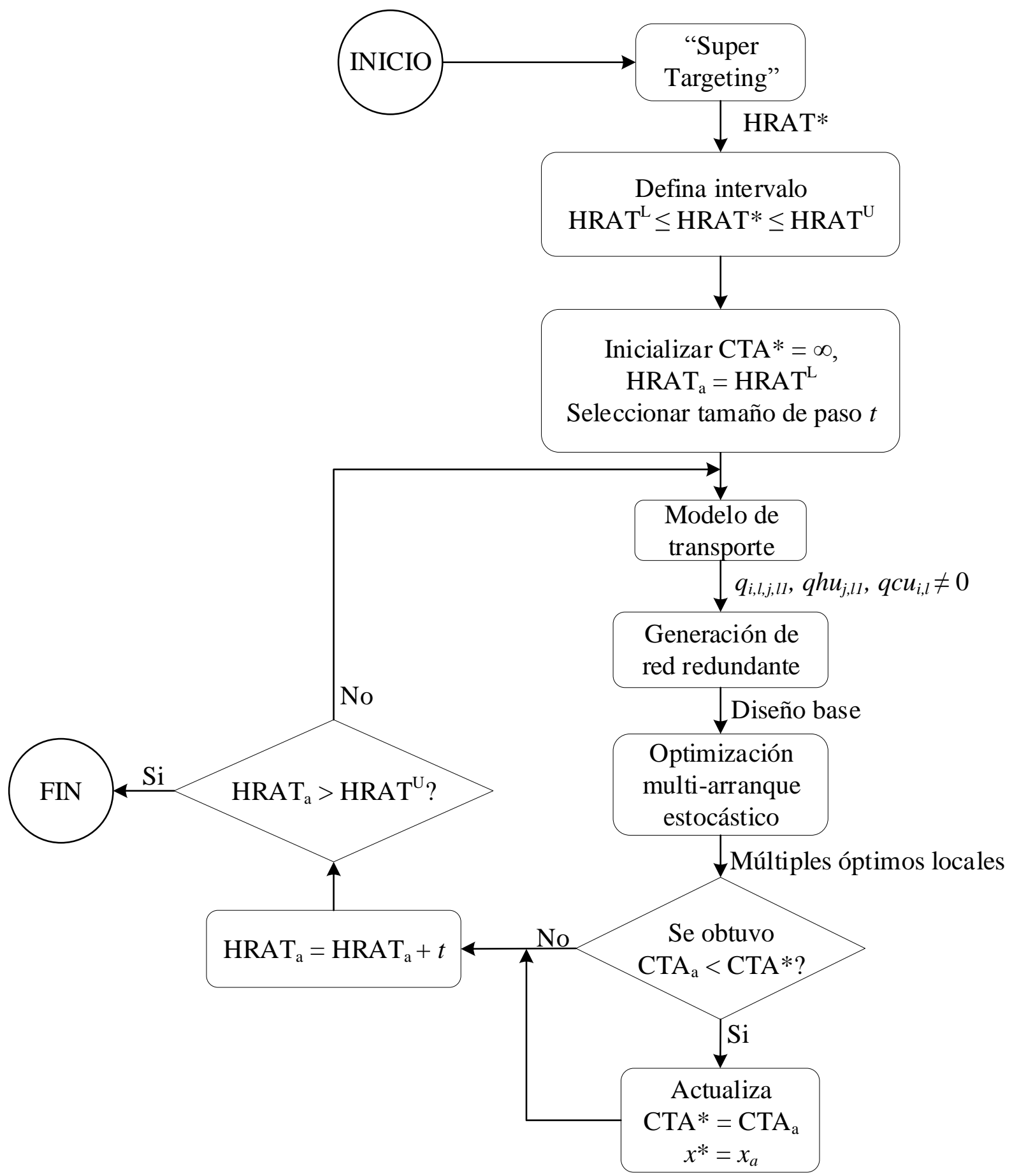

Figura 4.19. Algoritmo para la generación y optimización de topologías redundantes para la síntesis de redes de intercambio de calor. 
El segundo sub-problema consiste en la optimización del CTA del diseño de red inicial considerando únicamente su estructura topológica mediante el método de multi-arranque estocástico en dos fases (Núñez-Serna y Zamora, 2016). El enfoque de optimización permite, a partir de la inicialización aleatoria de las variables de decisión, llevar a cabo reducciones estructurales en el diseño inicial estableciendo una adecuada distribución de los valores de las variables de decisión. Es entonces posible determinar múltiples soluciones óptimas locales contenidas en una topología redundante dada, permitiendo seleccionar de entre ellas la que posea el menor CTA.

Para cada topología redundante optimizada se guarda registro si en alguna iteración se ha conseguido una solución de menor costo, actualizando tanto el vector que contiene a las variables de decisión de modo que $x^{*}=x_{a}$, como el costo de la mejor solución disponible en $\mathrm{CTA}^{*}=\mathrm{CTA}_{\mathrm{a}}$. En resumen, un conjunto de topologías redundantes son optimizadas para determinar múltiples soluciones óptimas locales las cuales son comparadas para seleccionar aquella que implique un mayor beneficio en términos del CTA.

La principal limitante de la metodología de síntesis propuesta se asocia al número de topologías redundantes que el diseñador propone evaluar. Generar numerosos diseños iniciales, así como disponer de un número considerable de inicializaciones en la metodología de optimización, incrementa las posibilidades de obtener soluciones de calidad, pero, por otro lado, se incrementa el impacto que se tiene en el uso de recursos computacionales destinado a la evaluación de todos los diseños base. 


\section{Capítulo 5. Resultados}

\subsection{Observaciones preliminares}

La metodología de síntesis de redes de intercambio de calor propuesta en este trabajo consiste en la generación y optimización de múltiples redes de intercambio de calor redundantes. Estas estructuras se pueden generar mediante la variación del parámetro HRAT que establece las diferentes rutas para la transferencia de calor mediante el problema de uso mínimo de servicios auxiliares formulado como un modelo de transporte. Otro factor que determina las características de la red de intercambio de calor redundante es la selección del tamaño o nivel de redundancia del diseño, utilizando las posibles reducciones de los elementos de la distribución de cargas térmicas desarrollados en el Capítulo 4.

Los esquemas para definir los equipos que serán asignados a un diseño base mediante las diferentes interpretaciones a la distribución de cargas térmicas se referirán como: i) el primer nivel de redundancia correspondiente a la Topología redundante 1 de la Sección 4.3, que asigna todos los elementos de la distribución de cargas térmicas a un intercambiador de calor generando el mayor número de equipos disponibles; ii) el segundo nivel de redundancia se refiere a la Topología redundante 2, el cual integra algunos apareamientos de la distribución de cargas térmicas para reducir el número de equipos; iii) el tercer nivel de redundancia correspondiente a la Topología redundante 3 permite un único intercambio de calor por corriente produciendo el número más conservador de equipos.

La importancia de definir el nivel de redundancia de los diseños base, se debe a que, en conjunto con el HRAT, son identificadores que proporcionan registro de las topologías redundantes que serán optimizadas, permitiendo distinguir aquellas que contengan a los óptimos locales con menor CTA. Tanto el nivel de redundancia, como el conjunto seleccionado de parámetros HRAT para la disposición de las múltiples topologías redundantes y el número iteraciones que se fijan en el método de multi-arranque estocástico en dos fases que definen las inicializaciones 
aleatorias para la determinación de óptimos locales, se consideran parámetros de entrada al igual que los datos del problema.

Los parámetros de entrada alimentan al programa computacional desarrollado para síntesis de redes de intercambio de calor mediante la generación y optimización de topologías redundantes. Dicho programa se desarrolló en el ambiente GAMS 21.6 y se resolvió en una computadora personal DELL XPS 8500 con CPU Intel i7-3770 3.7 GHz con 16 GB de memoria RAM. Para la solución del modelo de transporte se utilizó CPLEX v12.0 como resolvedor de PL. Así mismo, el tratamiento de datos de la distribución de cargas térmicas, establecido por el nivel de redundancia, se realiza de forma automática para generar la topología redundante, proporcionando los conjuntos y parámetros de entrada que son asignados al método de multiarranque estocástico en dos fases para la optimización de cada diseño base, utilizando CONOPT v3 como resolvedor de PNL. El programa es ejecutado a lo largo de un cierto número de iteraciones, dictadas por el conjunto seleccionado de parámetros HRAT, guardando en un archivo de salida los valores de las variables de decisión y el CTA de cada óptimo local determinado.

Para el desarrollo de este trabajo, el parámetro EMAT se fijó en $1{ }^{\circ} \mathrm{C}$ o $1{ }^{\circ} \mathrm{F}$ dependiendo el sistema de unidades de cada problema. La aproximación de Paterson (Paterson, 1984) es usada para sustituir la DMLT en el modelo de PNL con el fin de evitar problemas numéricos cuando las diferencias de temperaturas del lado caliente y frío de algún intercambiador de calor son iguales. Sin embargo, las soluciones finales son verificadas y reevaluadas con la DMLT rigurosa para realizar una comparación directa del CTA con los valores previamente reportados en la literatura. En los debidos casos donde se haga uso de alguna aproximación para la DMLT el costo de un diseño se indicará de forma explícita por medio de un subíndice (e.g. CTA Paterson). En caso contrario se asumirá que el costo corresponde al uso de la DMLT rigurosa.

En este trabajo se abordan siete casos de estudio extraídos de la literatura para mostrar la eficacia de la metodología secuencial de síntesis de redes de intercambio de calor. Los casos de estudio seleccionados poseen diferentes tamaños con el fin de examinar el alcance del enfoque propuesto. A continuación, se muestran los resultados obtenidos para cada caso de estudio. 


\subsection{Caso de Estudio 5.1}

El primer caso de estudio fue propuesto por Zamora y Grossmann (1997) el cual consiste en la integración térmica de dos corrientes calientes y dos corrientes frías, este problema se utilizó como ejemplo ilustrativo en capítulos anteriores y a partir de ahora se referirá al mismo, como el Caso de Estudio 5.1. En la Tabla 5.1 se describen los datos de corrientes, servicios auxiliares y costos del problema.

En el trabajo desarrollado por Zamora y Grossmann (1997) se aborda la síntesis de redes de intercambio de calor mediante un enfoque de optimización global determinista utilizando planos que acotan la DMLT mediante una premisa de diseño sin división de corrientes. El valor de la función objetivo determinado por los autores corresponde a un CTA de \$419,979.

Tabla 5.1. Datos de corrientes y costos para el Caso de Estudio 5.1.

\begin{tabular}{|c|c|c|c|c|}
\hline Corriente & $\begin{array}{l}\text { Temperatura de } \\
\text { suministro } \\
\operatorname{Ts}\left({ }^{\circ} \mathrm{C}\right)\end{array}$ & $\begin{array}{c}\text { Temperatura } \\
\text { objetivo } \\
\text { To }\left({ }^{\circ} \mathrm{C}\right)\end{array}$ & $\begin{array}{c}\text { Flujo de capacidad } \\
\text { calorífica } \\
\mathrm{F}\left(\mathrm{kW}^{\circ} \mathrm{C}^{-1}\right)\end{array}$ & $\begin{array}{l}\text { Coeficiente de } \\
\text { película } \\
\mathrm{h}\left(\mathrm{kW} \mathrm{m}^{-2}{ }^{\circ} \mathrm{C}^{-1}\right)\end{array}$ \\
\hline H1 & 180 & 75 & 30 & 0.15 \\
\hline $\mathrm{H} 2$ & 240 & 60 & 40 & 0.10 \\
\hline $\mathrm{C} 1$ & 40 & 230 & 35 & 0.20 \\
\hline $\mathrm{C} 2$ & 120 & 300 & 20 & 0.10 \\
\hline $\mathrm{HU}$ & 325 & 325 & - & 2 \\
\hline $\mathrm{CU}$ & 25 & 40 & - & 0.50 \\
\hline
\end{tabular}

Costo de intercambiadores de calor y enfriadores $\left(\$\right.$ año $\left.^{-1}\right)=1500+30\left[\text { Área }\left(\mathrm{m}^{2}\right)\right]^{0.8}$

Costo de calentadores $\left(\$ a\right.$ año $\left.^{-1}\right)=1500+60\left[\text { Área }\left(\mathrm{m}^{2}\right)\right]^{0.8}$

Costo de servicios de calentamiento $=110\left(\$ \mathrm{~kW}^{-1} \mathrm{año}^{-1}\right)$

Costo de servicios de enfriamiento $=10\left(\$ \mathrm{~kW}^{-1} \mathrm{año}^{-1}\right)$

Björk y Westerlund (2002) retoman posteriormente el problema mediante un enfoque riguroso de optimización global utilizando la aproximación de Paterson (Paterson, 1984) para la DMLT, pero permitiendo divisiones de corriente y estableciendo redes de intercambio de calor con y sin la restricción de mezclado isotérmico. Estos autores presentan un diseño de red con un CTA $\mathrm{A}_{\text {Paterson }}$ de $\$ 415,189$ y también afirman en la sección de datos de problemas, que la mejor solución 


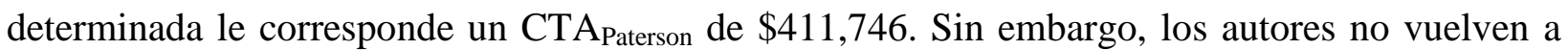
mencionar esta solución en el apartado de resultados y tampoco presentan el diseño de red correspondiente, en consecuencia, se asume que la mejor solución obtenida por Björk y Westerlund (2002) se atribuye al diseño con un CTAPaterson de \$415,189. Las propuestas anteriores toman como base la superestructura del modelo Synheat (Yee y Grossmann, 1990), donde los diseños finales de ambos autores presentan cuatro intercambiadores de calor, un calentador y un enfriador.

\section{Elección de parámetros de entrada}

Para la metodología propuesta en este trabajo, el parámetro HRAT juega un papel importante para el diseño de las múltiples topologías redundantes que puedan ser optimizadas. El procedimiento de "Super-Targeting" (Linnhoff y Hindmarsh, 1983) permite estimar los costos previamente al diseño de una red de intercambio de calor para diferentes HRAT. Mediante esta técnica es posible identificar el HRAT que produce la mínima estimación del CTA. En la Figura 5.1 se muestra que, mediante el procedimiento de "Super-Targeting", el HRAT óptimo para el Caso de Estudio 5.1 corresponde a un valor de $4{ }^{\circ} \mathrm{C}$. Así mismo en la Tabla 5.2 se detallan los valores de recuperación de calor y uso de servicios auxiliares para la estimación del CTA.

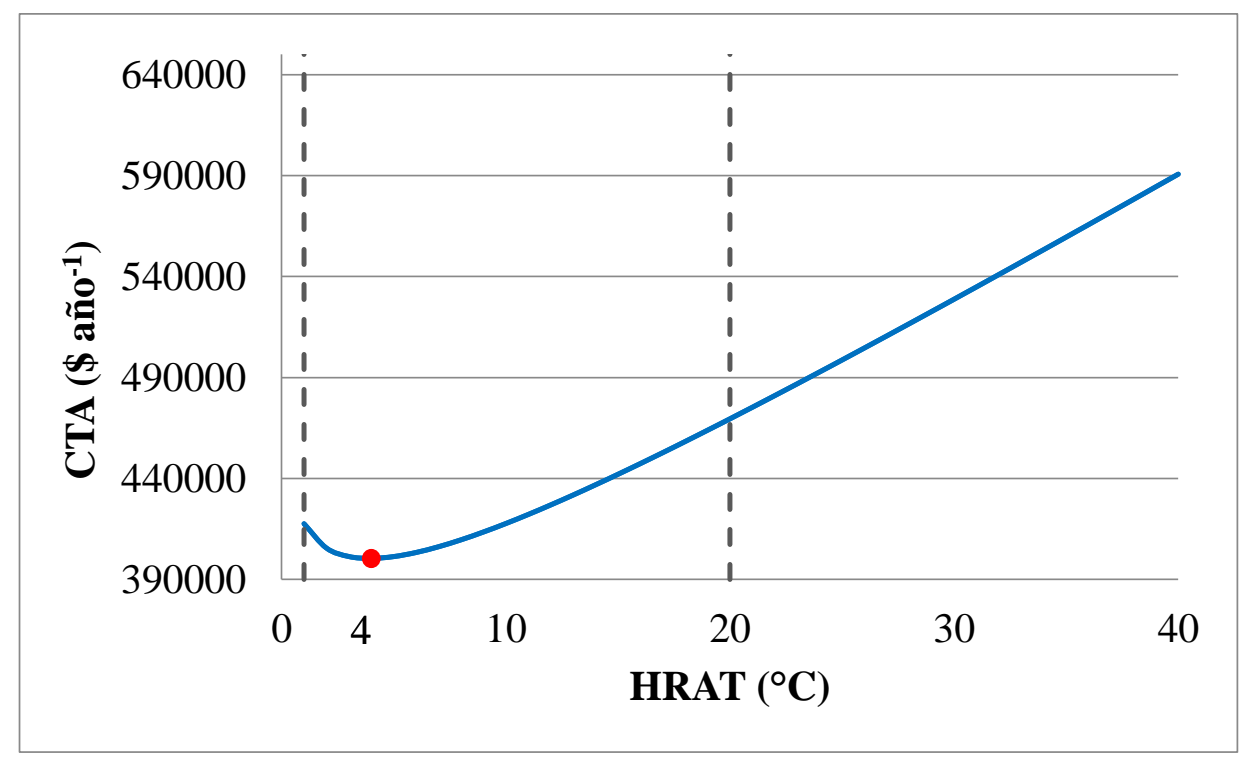

Figura 5.1. "Super-targeting” y selección del intervalo de HRAT para la generación de topologías redundantes en el Caso de Estudio 5.1. 
Tabla 5.2. Valores estimados por el método de "Super-Targeting" para el Caso de Estudio 5.1.

\begin{tabular}{ccccc}
\hline $\begin{array}{c}\text { HRAT } \\
\left({ }^{\circ} \mathrm{C}\right)\end{array}$ & $\begin{array}{c}\text { Calor } \\
\text { recuperado } \\
\mathrm{q}_{\mathrm{rec}}(\mathrm{kW})\end{array}$ & $\begin{array}{c}\text { Servicios de } \\
\text { calentamiento } \\
\mathrm{qhu}(\mathrm{kW})\end{array}$ & $\begin{array}{c}\text { Servicios de } \\
\text { enfriamiento } \\
\mathrm{qcu}(\mathrm{kW})\end{array}$ & $\begin{array}{c}\text { CTA } \\
\text { estimado } \\
\left(\$ \text { año }^{-1}\right)\end{array}$ \\
\hline 1 & 8445 & 1805 & 1905 & $417,599.30$ \\
3 & 8335 & 1915 & 2015 & $401,356.57$ \\
$\mathbf{4}$ & $\mathbf{8 2 8 0}$ & $\mathbf{1 9 7 0}$ & $\mathbf{2 0 7 0}$ & $\mathbf{4 0 0 , 4 3 4 . 2 7}$ \\
5 & 8225 & 2025 & 2125 & $401,324.47$ \\
10 & 7950 & 2300 & 2400 & $417,682.56$ \\
20 & 7400 & 2850 & 2950 & $469,532.76$ \\
\hline
\end{tabular}

Debido a las características sistemáticas de la metodología puede resultar favorable evaluar un número considerable de topologías redundantes que incrementen las posibilidades de determinar diferentes soluciones con CTA competitivo, esto se logra eligiendo un conjunto de parámetros cercanos al HRAT óptimo, con la premisa de que las topologías redundantes asociadas a estos parámetros guarden alguna relación con la estimación de costos otorgada por el método de "Super-Targeting".

Para el Caso de Estudio 5.1 se elige un intervalo de exploración contenido entre el intervalo de un $\operatorname{HRAT}^{\mathrm{L}}=1{ }^{\circ} \mathrm{C}$ y un $\operatorname{HRAT}^{\mathrm{U}}=20^{\circ} \mathrm{C}$ con un tamaño de paso $t=1{ }^{\circ} \mathrm{C}$, lo que significa que se iniciará la generación de topologías redundantes desde un HRAT de $1{ }^{\circ} \mathrm{C}$ incrementando en cada iteración un grado al HRAT hasta llegar a $20{ }^{\circ} \mathrm{C}$, lo anterior se puede interpretar como veinte distribuciones de cargas térmicas que pueden generar topologías redundantes. A su vez las distribuciones de cargas térmicas pueden ser tratadas mediante los tres niveles de redundancia disponibles, por lo que en total para el Caso de Estudio 5.1 se generarán y optimizarán 60 diseños base.

Para cada estructura redundante a evaluar, la metodología de optimización requiere que se fije el número de inicializaciones para la determinación de óptimos locales. Este número se traduce en el tiempo de cómputo que se destinará a la optimización de cada diseño base redundante. Un número grande implicará una menor incertidumbre con respecto a la evaluación de los diferentes óptimos locales que están contenidos en una topología redundante, pero un gasto computacional importante. Por otro lado, con un número pequeño de inicializaciones, aunque signifique menor tiempo de cómputo invertido, se incrementa la posibilidad de omitir algún óptimo local de 
calidad. En cada caso de estudio se debe buscar un número de iteraciones que guarde un balance entre un bajo riesgo en la omisión de óptimos locales, sin la necesidad de una inversión excesiva de recursos computacionales.

Para la elección del número de inicializaciones se puede realizar un análisis sobre una única topología redundante incrementando gradualmente el número de éstas y tomando registro en donde ya no surgen nuevos óptimos locales. Para el Caso de Estudio 5.1, por ejemplo, se elige una topología redundante construida usando el HRAT óptimo de $4{ }^{\circ} \mathrm{C}$ y el primer nivel de redundancia. La propuesta de generación propone una topología redundante con un total de 14 intercambiadores de calor, en la Figura 5.2 se muestra el número de iteración en la que por primera vez se detecta una solución óptima local. Se puede observar que después de realizar 1000 inicializaciones determinando factibilidad en cada una, se identifican 21 soluciones óptimas locales diferentes donde la última es revelada en la iteración 213, esto significa que la mayoría de las iteraciones se malgastaron en la determinación de óptimos locales repetidos.

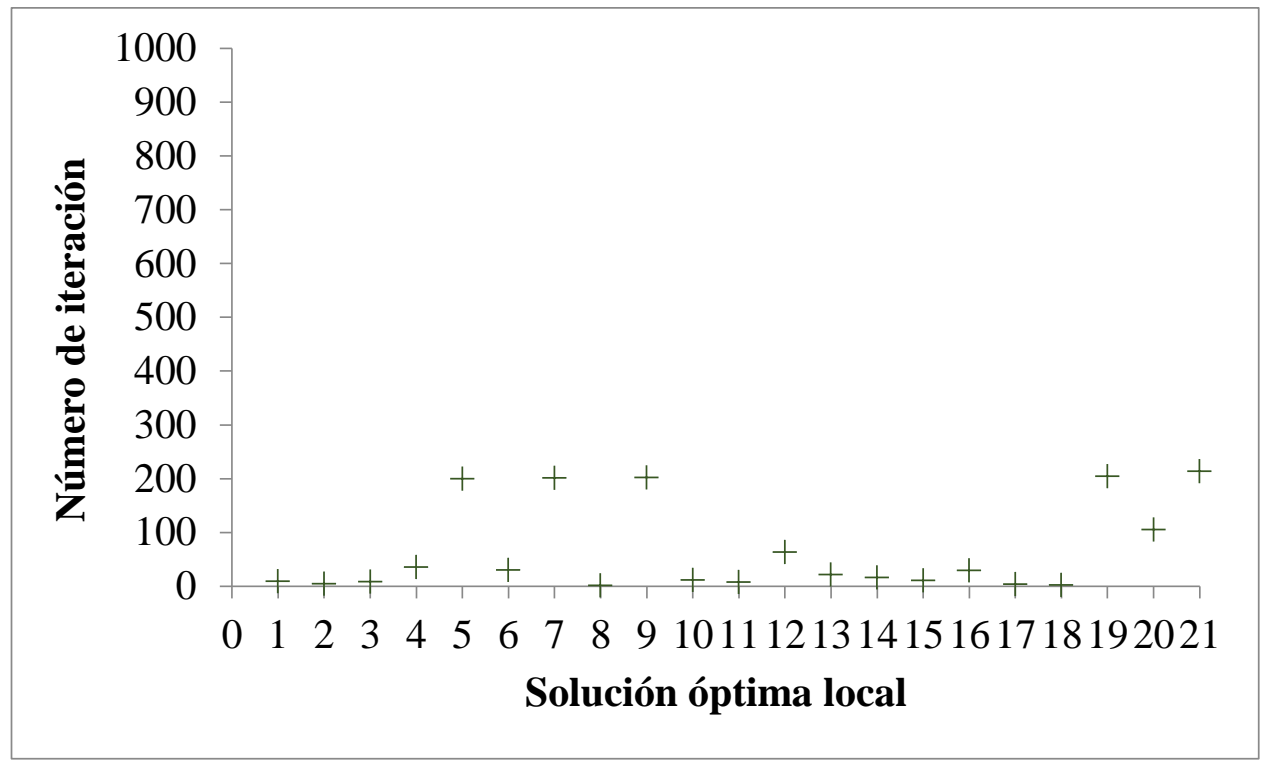

Figura 5.2. Primera ocurrencia de cada solución óptima local, de la optimización de una topología redundante con el tercer nivel de redundancia y HRAT de $4{ }^{\circ} \mathrm{C}$ realizando 1000 inicializaciones para el Caso de Estudio 5.1.

Si se consideran estos resultados como una medida representativa de las diferentes topologías redundantes a generar, se considera seleccionar 250 como un número razonable de inicializaciones para el Caso de Estudio 5.1. Es importante tener presente que lo anterior es solo 
un indicador que sugiere la intensidad de la búsqueda con el objetivo de ahorrar recursos computacionales, mas no un criterio formal que impida realizar una exploración exhaustiva si se desea asegurar que no se han omitido soluciones óptimas locales. En la Sección 3.5 se discutieron las diferencias que pueden exhibir topologías redundantes incluso con características similares, cada una presentando diferentes cantidades de óptimos locales y una reincidencia baja en algunos de ellos, por lo que el número de inicializaciones es un factor de riesgo donde la experiencia del diseñador juega el papel más importante.

\section{Generación y optimización de topologías redundantes}

Una vez elegidos los parámetros de entrada, se procede a la metodología de síntesis de redes de intercambio de calor mediante la generación y optimización de topologías redundantes. En la Tabla 5.3 se detallan algunos resultados estadísticos que representan la prueba realizada para los tres niveles de redundancia en el Caso de Estudio 5.1. Se puede apreciar una clara relación entre el tamaño de las topologías redundantes y la cantidad de óptimos locales determinados, siendo el primer nivel, el que otorga más equipos y por consecuencia los diseños base que contienen una mayor cantidad de óptimos locales. Así mismo esta característica también asocia un tiempo de cómputo más elevado al incluir más variables y restricciones en comparación con las estructuras iniciales de menor tamaño.

Tabla 5.3. Resultados del Caso de Estudio 5.1.

Nivel de redundancia Primer nivel Segundo nivel Tercer nivel

\begin{tabular}{lccc}
\hline Topologías redundantes generadas & 20 & 20 & 20 \\
Número de inicializaciones por diseño base & 250 & 250 & 250 \\
Soluciones factibles determinadas & 4955 & 4750 & 4750 \\
Óptimos locales diferentes & 90 & 54 & 29 \\
Promedio de equipos en topologías redundantes & 13 & 9 & 8 \\
Promedio de equipos en diseños óptimos & 6 & 6 & 5 \\
Tiempo de CPU promedio por inicialización & $0.01004 \mathrm{~s}$ & $0.00745 \mathrm{~s}$ & $0.00644 \mathrm{~s}$ \\
Tiempo total de CPU & $50.203 \mathrm{~s}$ & $37.266 \mathrm{~s}$ & $32.219 \mathrm{~s}$ \\
\hline
\end{tabular}

En la Figura 5.3 se muestran las mejores soluciones para cada HRAT, utilizando los tres diferentes niveles de redundancia disponibles. Para el Caso de Estudio 5.1, que puede 
considerarse como un problema de pequeña escala, es posible observar que en repetidas ocasiones se incide sobre los mismos óptimos locales, lo que se puede deber a que a pesar de que la distribución de cargas térmicas es diferente en cada HRAT, la metodología de generación pudiera coincidir en definir la misma topología redundante. Otra razón se debe a que a pesar de tener topologías redundantes que presenten diferencias estructurales, al eliminar ciertos equipos mediante el enfoque de optimización la mejor solución converja en la misma red de intercambio de calor simplificada.

Las soluciones obtenidas que presentan el menor costo corresponden a un $\mathrm{CTA}_{\text {Paterson }}$ $\$ 417,407.42$ por año (línea punteada) con la aproximación de Paterson, mientras que la mejor solución reportada en la literatura presenta un $\mathrm{CTA}_{\text {Paterson }}$ de $\$ 415,189$ (línea sólida) determinada por Björk y Westerlund (2002). Los óptimos locales con el menor costo son determinados en las estructuras generadas con un HRAT de $20{ }^{\circ} \mathrm{C}$ tanto para el segundo y tercer nivel de redundancia. Resulta interesante mencionar que la mejor solución obtenida se generó en el HRAT más alejado al estimado como óptimo por la metodología de "Super-Targeting", lo que sugiere que, en algunos casos cualquier topología redundante puede tener posibilidades de determinar soluciones atractivas puesto que la metodología de optimización considera únicamente los elementos estructurales del diseño base.

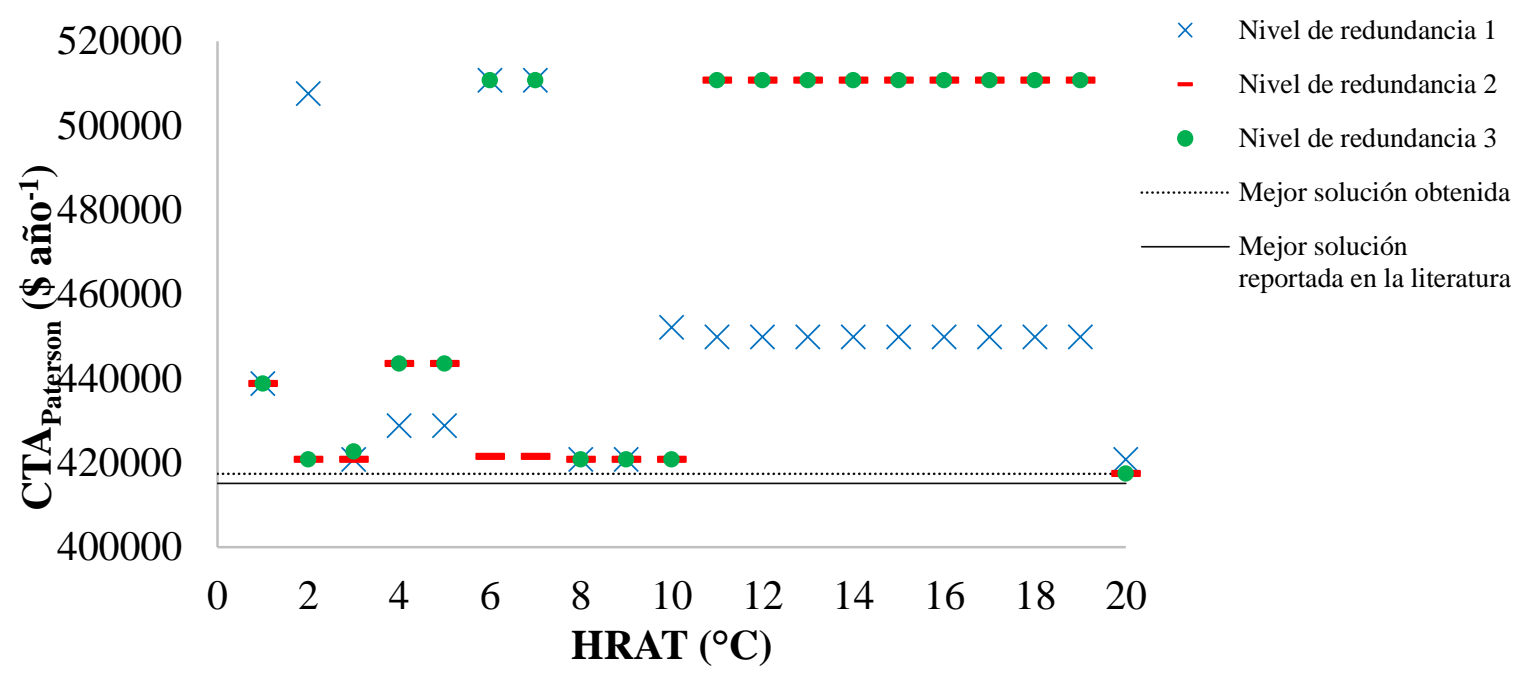

Figura 5.3. Óptimos locales de menor CTA Paterson para cada HRAT en el Caso de Estudio 5.1.

La determinación de soluciones competitivas está fuertemente relacionada con las características que posee la topología redundante a optimizar, por esta razón realizar una comparación de las 
soluciones con mayor costo pudiera exponer las particularidades que llevan a la metodología de optimización a caer en óptimos locales indeseables. En la Figura 5.4 se aprecia la solución que presenta el costo más elevado, resultante de las pruebas realizadas y generada con un HRAT de 6 ${ }^{\circ} \mathrm{C}$ usando el primer nivel de redundancia con un CTA $\mathrm{Aaterson}_{\text {de }} \$ 510,696$. Cabe recordar que esta solución representa el mejor óptimo local del diseño base, construido con los parámetros mencionados, lo que significa que otras soluciones resultantes de la optimización de dicha estructura pudieran presentar un costo aún más elevado.

En la Tabla 5.4 se describe la comparación entre soluciones representativas que exhiben diferencias importantes en su costo. En las primeras dos filas se muestra las mejores dos soluciones obtenidas hasta el momento. En las filas siguientes se muestran el mejor y el peor óptimo local del diseño base generado con un HRAT de $6^{\circ} \mathrm{C}$ y el primer nivel de redundancia, el cual generó el óptimo local con el mayor CTA en comparación con los mostrados en la Figura 5.3. Se puede apreciar que algunos óptimos locales con costos poco atractivos exhiben una menor recuperación de calor en comparación con las soluciones de menor costo, requiriendo en ambos casos un calentador de más, por lo que estas soluciones pueden asociarse a un alto uso de servicios auxiliares.

Tabla 5.4. Comparación de las mejores soluciones obtenidas con soluciones de alto costo para el Caso de Estudio 5.1.

\begin{tabular}{ccccc}
\hline $\begin{array}{c}\mathrm{CTA}_{\text {Paterson }} \\
\left(\$ \text { año }^{-1}\right)\end{array}$ & Equipos & $\begin{array}{c}\text { Calentadores } \\
\text { Enfriadores }\end{array}$ & $\begin{array}{c}\text { Calor } \\
\text { recuperado } \\
\mathrm{q}_{\mathrm{rec}}(\mathrm{kW})\end{array}$ & $\begin{array}{c}\text { Área } \\
\mathrm{A}\left(\mathrm{m}^{2}\right)\end{array}$ \\
\hline $417,407.42$ & 6 & $\begin{array}{c}\mathrm{HU} 2 \\
\mathrm{CU} 2\end{array}$ & 8125.40 & 11532.8 \\
$420,699.51$ & 6 & $\begin{array}{c}\mathrm{HU} 2 \\
\mathrm{CU} 1, \mathrm{CU} 2\end{array}$ & 8096.02 & 12439.11 \\
$510,696.76$ & 6 & $\begin{array}{c}\mathrm{HU} 1, \mathrm{HU} 2 \\
\mathrm{CU} 2\end{array}$ & 7227.83 & 8127.23 \\
$681,942.69$ & 5 & $\begin{array}{c}\mathrm{HU} 1, \mathrm{HU} 2 \\
\mathrm{CU} 2\end{array}$ & 5525.29 & 4988.17 \\
\hline
\end{tabular}




\section{Generación y optimización de topologías redundantes restringiendo servicios auxiliares}

Con el fin de explorar diseños alternativos que presenten características que favorezcan una eficiente recuperación de calor y que se traduzcan en redes de intercambio de calor con mejores oportunidades de presentar costos competitivos, se propone imponer restricciones en los apareamientos sobre la formulación del modelo de transporte para limitar el calentamiento y enfriamiento en sobre algunas corrientes de proceso.

Como punto de partida se imponen las condiciones con las cuales se generó la mejor solución obtenida, asignando un alto costo de transporte a los apareamientos que se desea evitar de forma que $C C U_{1, l}=M$ y $C H U_{1, l 1}=M$, donde $M$ es un número muy grande. Lo anterior implica que al resolver el modelo de transporte se optará por impedir las rutas de transferencia de calor entre las corrientes de proceso $\mathrm{H} 1$ y $\mathrm{C} 1$ con sus respectivos servicios auxiliares, generando diseños base que no incluyan calentadores o enfriadores sobre dichas corrientes según sea el caso.

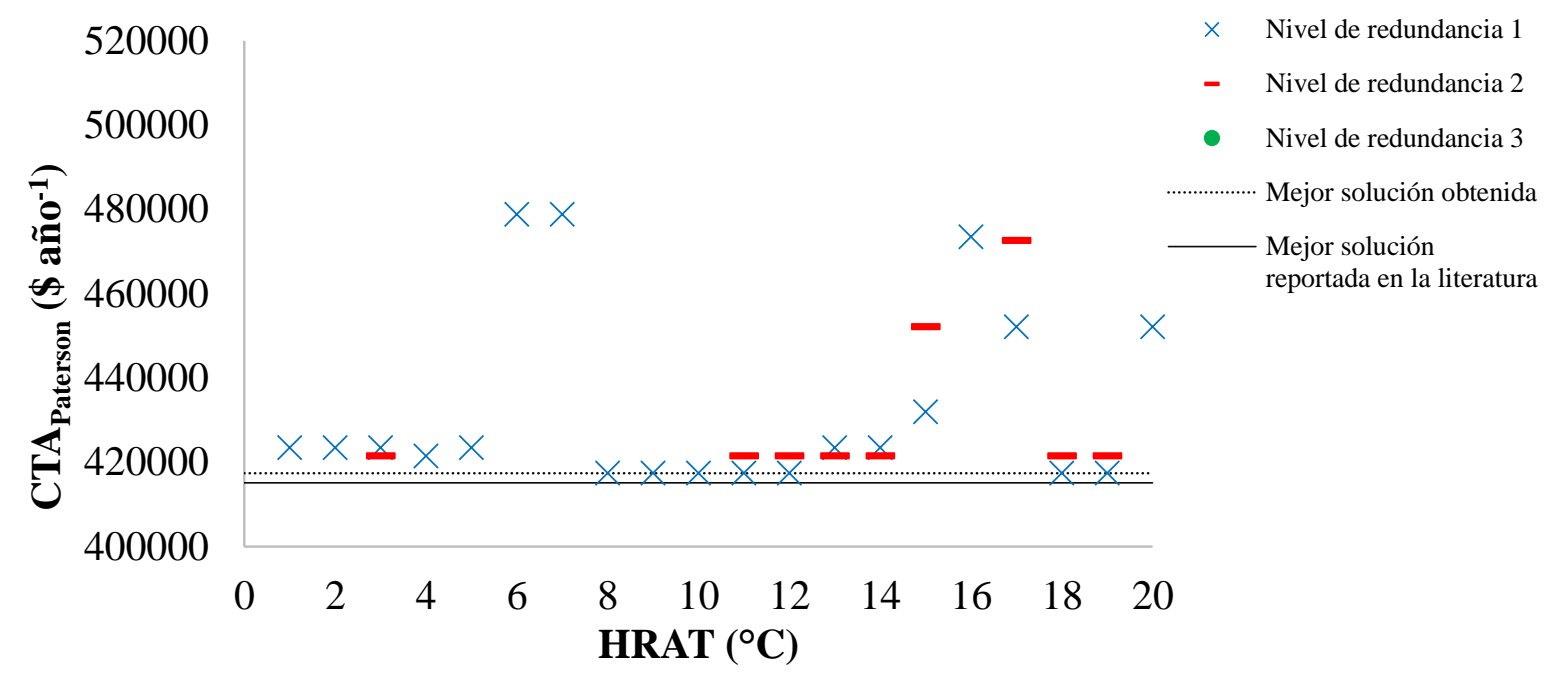

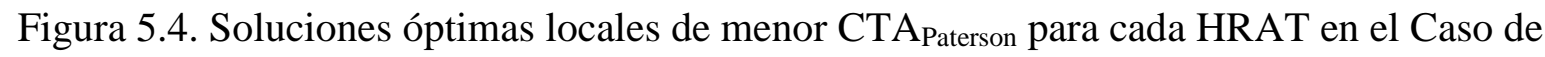

Estudio 5.1, prohibiendo los servicios auxiliares a las corrientes de proceso $\mathrm{H} 1$ y C1.

En la Figura 5.4 se observan los óptimos locales con menor costo para cada HRAT bajo las consideraciones impuestas. La mejor solución continúa mostrando un CTA Paterson de $\$ 417,407.42$, sin embargo, se puede apreciar que, en el primer nivel de redundancia, dicha solución reincide con mayor frecuencia en comparación de cuando se permitió que la solución del modelo de transporte determinara los servicios auxiliares. Por otro lado, se debe mencionar que en el tercer nivel de redundancia no se obtuvo ninguna solución factible mientras que en el 
segundo nivel, en ciertos HRAT se presenta la misma situación, esto se debe a que la integración de apareamientos producto de las formulaciones de reducción de los elementos de la distribución de cargas térmicas, impiden que las topologías redundantes posean suficientes equipos para llevar a cabo una integración térmica adecuada, sumado a que la eliminación de calentadores y enfriadores pudiera inhabilitar la posibilidad de que las corrientes de proceso alcancen sus temperaturas objetivo mediante el uso de servicios auxiliares.

Como se mostró en la Figura 5.4, debido al tamaño de este problema, prohibir ciertos servicios auxiliares puede resultar decisivo al momento de determinar de soluciones factibles. Por estas razones y debido a que la inversión computacional es relativamente modesta en problemas de este tamaño, resulta aceptable realizar diferentes pruebas con una distinta distribución de servicios auxiliares. Se propone ahora una mantener el servicio de calentamiento en la corriente C1, y cambiar la prohibición en el servicio de enfriamiento a la corriente H2 de forma que $C C U_{2, l}=M$ y $C H U_{1, l 1}=M$. En la Tabla 5.5 se describen las estadísticas para la prueba realizada restringiendo servicios auxiliares elegidos, la principal diferencia que se observa en estos resultados, es que se generan una menor cantidad de óptimos locales diferentes en comparación con la primera prueba realizada sin limitar el uso de calentamiento y enfriamiento.

Tabla 5.5. Resultados del Caso de Estudio 5.1 prohibiendo servicios auxiliares a las corrientes de proceso $\mathrm{H} 1$ y $\mathrm{C} 2$.

\begin{tabular}{lccc} 
& Primer nivel & Segundo nivel & Tercer nivel \\
\hline Topologías redundantes generadas & 20 & 20 & 20 \\
Número de inicializaciones por diseño base & 250 & 250 & 250 \\
Soluciones Factibles determinadas & 4014 & 4485 & 4995 \\
Óptimos locales diferentes & 30 & 18 & 5 \\
Promedio de equipos en topologías redundantes & 13 & 7 & 6 \\
Promedio de equipos en diseños óptimos & 6 & 5 & 5 \\
Tiempo de CPU promedio por inicialización & $0.01051 \mathrm{~s}$ & $0.00694 \mathrm{~s}$ & $0.00493 \mathrm{~s}$ \\
Tiempo total de CPU & $52.543 \mathrm{~s}$ & $34.954 \mathrm{~s}$ & $24.641 \mathrm{~s}$ \\
\hline
\end{tabular}




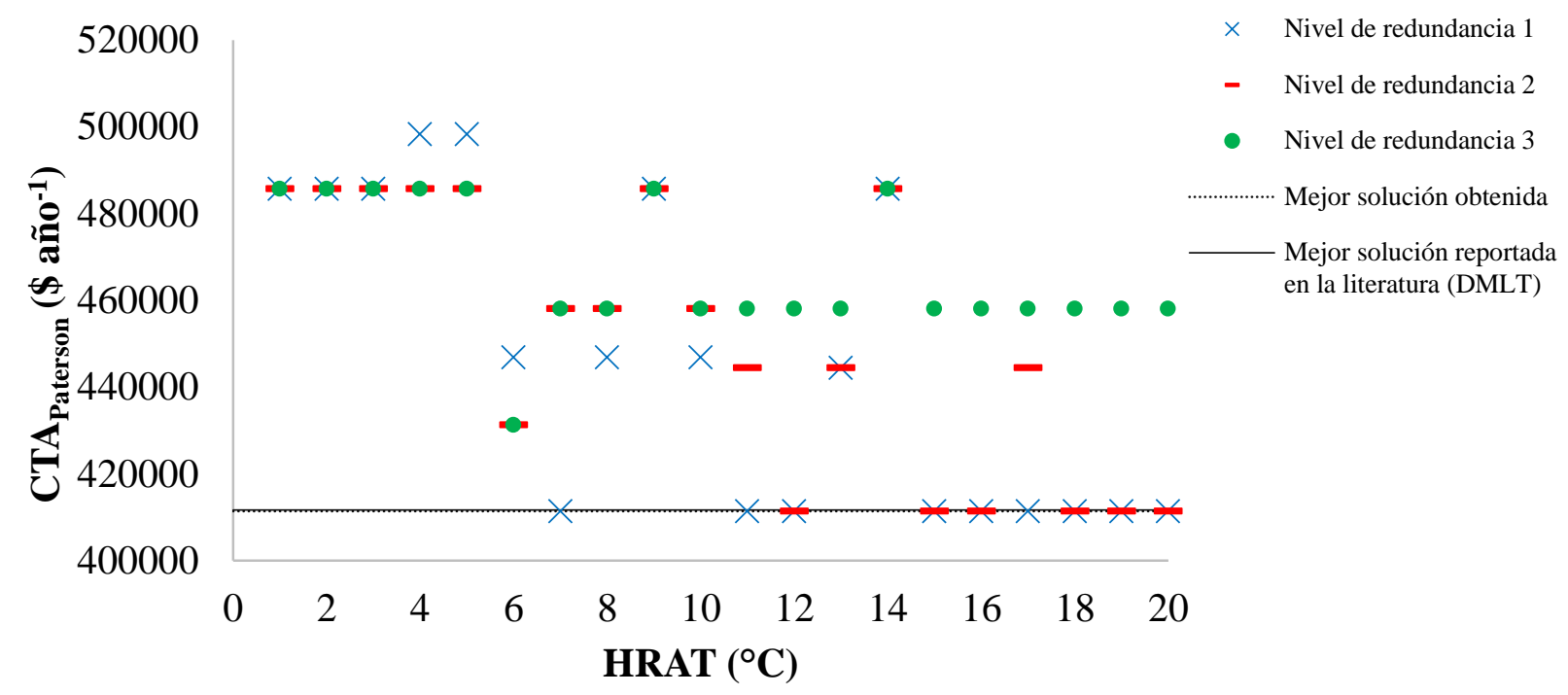

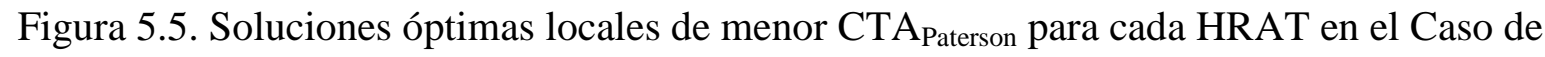

Estudio 5.1 prohibiendo los servicios auxiliares a las corrientes de proceso $\mathrm{H} 1$ y C2.

En la Figura 5.5 se muestran los óptimos locales de menor costo que corresponden a cada HRAT. Mediante los criterios considerados es posible diferenciar una nueva solución con CTA $_{\text {Paterson }}$ de $\$ 411,388.05$, dicha solución se determina en múltiples HRAT mediante el primer y segundo nivel de redundancia.

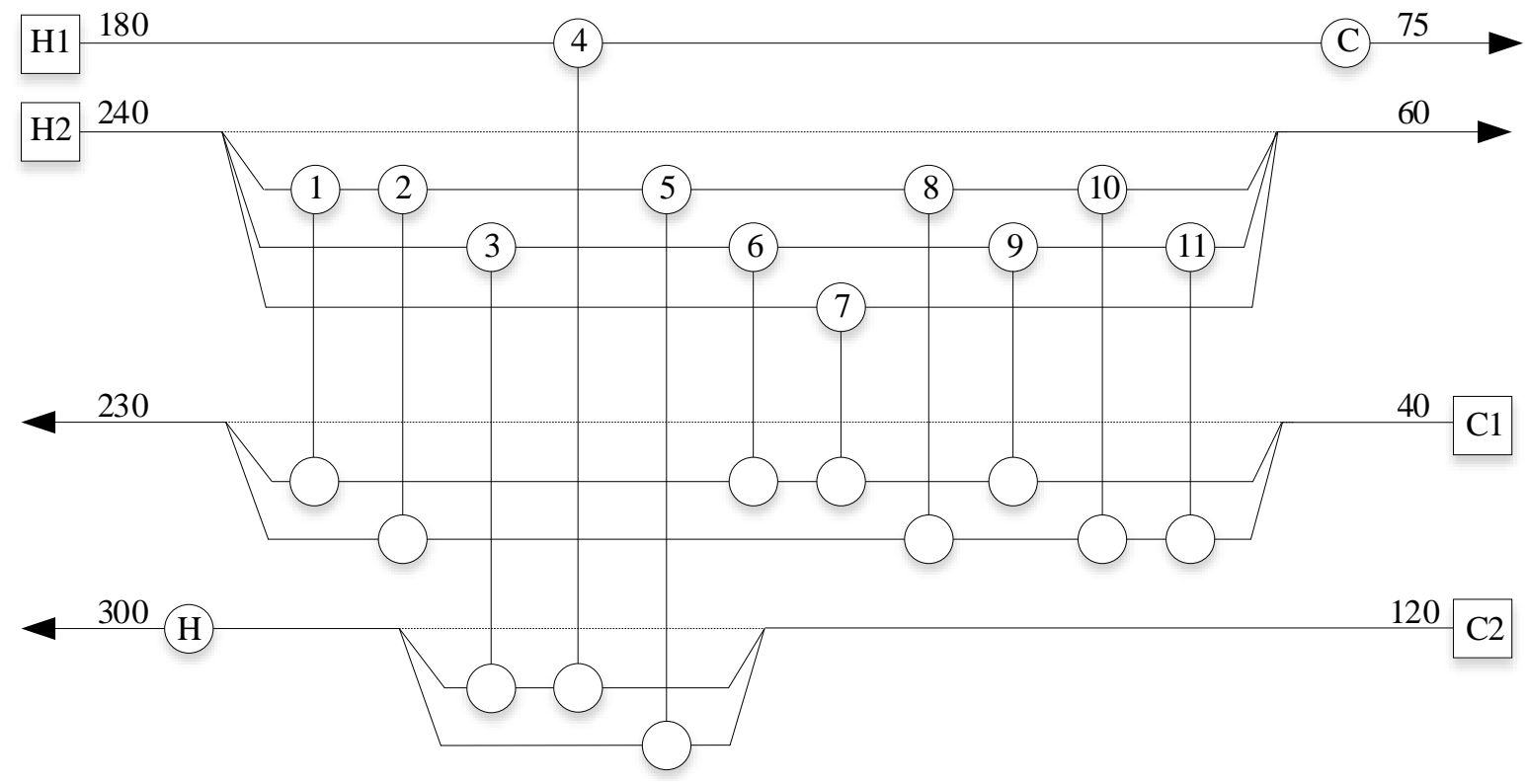

Figura 5.6. Topología redundante generada con un HRAT de $7{ }^{\circ} \mathrm{C}$ y el primer nivel de redundancia para el Caso de Estudio 5.1. 
Para ilustrar el proceso de generación y optimización de una de las topologías redundantes que determinaron la mejor solución disponible se elige el diseño base generado con el primer nivel de redundancia y un HRAT de $7{ }^{\circ} \mathrm{C}$. En Figura 5.6 se presenta el diagrama de malla de la topología redundante seleccionada, la cual muestra 13 equipos de intercambio de calor de los cuales 11 son recuperadores. El planteamiento de optimización para el diseño base planteado requiere 229 variables y 187 restricciones en el modelo de PNL del algoritmo de multi-arranque estocástico en dos fases.

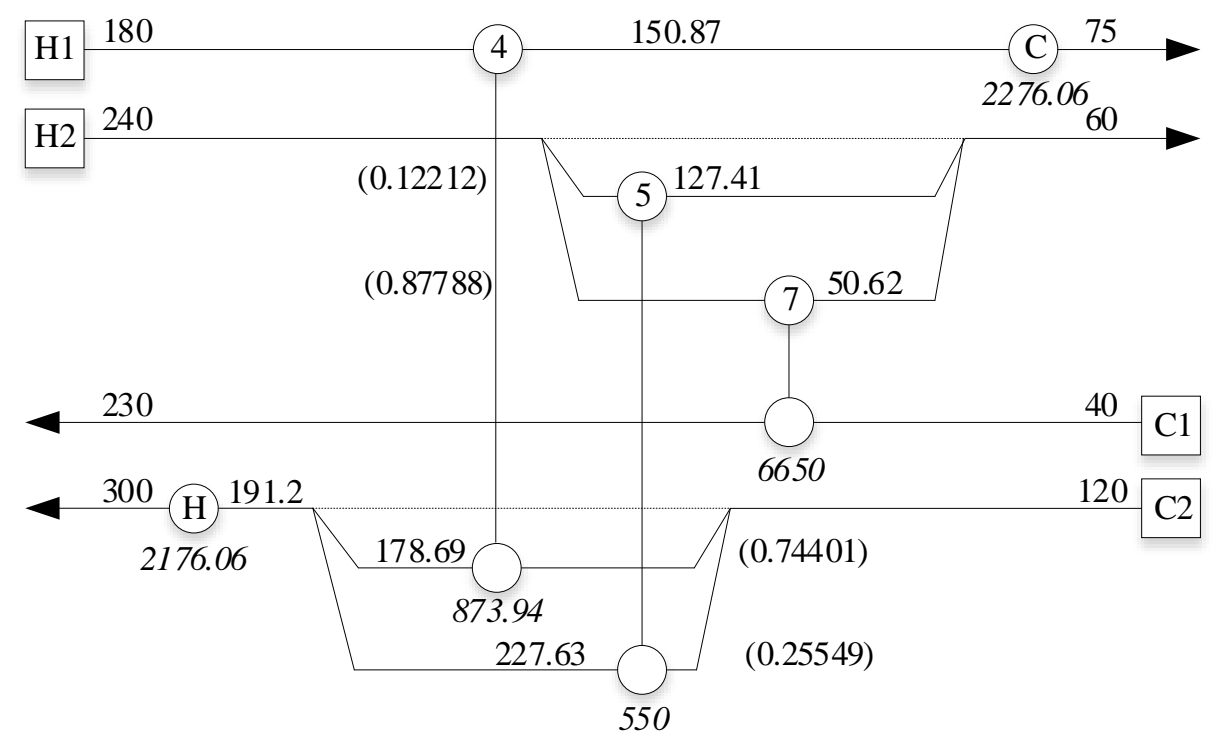

Figura 5.7. Mejor solución óptima local para el Caso de Estudio 5.1 con CTA de $\$ 411,624.48$ (temperaturas $\left({ }^{\circ} \mathrm{C}\right)$ en fuente normal, cargas térmicas $(\mathrm{kW})$ en cursivas y fracciones de flujo entre paréntesis).

En la Figura 5.7 se muestra la estructura optimizada con menor costo obtenida contenida en la topología redundante, la cual presenta un CTA $A_{\text {Paterson }}$ de $\$ 411,388.05$. Dentro de los círculos se indican las etiquetas de los equipos que no son eliminados por la técnica de optimización. La solución determinada en este trabajo se encuentra un $0.86 \%$ por debajo de la mejor solución reportada en la literatura determinada por Björk y Westerlund (2002), ambas utilizando la aproximación de Paterson (Paterson, 1984). La solución finalmente se reevalúa con la DMLT rigurosa, alcanzando a un costo de $\$ 411,624.48$ por año. Cabe mencionar que, aunque la red de intercambio de calor sintetizada en este trabajo si puede ser obtenida mediante la metodología de optimización global desarrollada por Björk y Westerlund (2002) los autores no presentan un diseño de red con las características de la solución obtenida en este trabajo. 


\section{Comparación de resultados}

Para el Caso de Estudio 5.1, en la Tabla 5.6 se muestra un resumen de comparación entre las soluciones desarrolladas por otros autores y la mejor solución determinada en este trabajo.

Tabla 5.6. Comparación del Caso de Estudio 5.1 con soluciones reportadas en la literatura.

\begin{tabular}{|c|c|c|c|}
\hline Autor(es) & Metodología & Configuración ${ }^{1}$ & $\begin{array}{c}\text { CTA Reportado } \\
\qquad \text { año }\end{array}$ \\
\hline $\begin{array}{l}\text { Zamora y Grossmann } \\
(1997)^{2}\end{array}$ & $\begin{array}{c}\text { Superestructura- } \\
\text { Optimización Global }\end{array}$ & $4,1,1-0(0)$ & 419,979 \\
\hline Björk y Westerlund (2002) ${ }^{2}$ & $\begin{array}{c}\text { Superestructura - } \\
\text { Optimización Global }\end{array}$ & $4,1,1-1(2)$ & $415,189 *$ \\
\hline Este trabajo & $\begin{array}{c}\text { Topologías } \\
\text { redundantes }\end{array}$ & $3,1,1-1(2)$ & $411,624.48$ \\
\hline
\end{tabular}

\subsection{Caso de Estudio 5.2}

El Caso de Estudio 5.2 fue presentado por Zamora y Grossmann (1998a) el cual consiste en tres corrientes de proceso calientes y dos frías con una función de costos lineal; la información del problema se muestra en la Tabla 5.7. Los autores proponen una metodología de optimización global determinista sin división de corrientes y sustituyendo la DMLT por la diferencia media aritmética de temperaturas (DMAT) obteniendo un diseño final con CTA de \$82,043 cuya reevaluación con la DMLT alcanza un costo de $\$ 83,400$ por año.

Diferentes autores han abordado el problema aplicando diversas metodologías de síntesis. Lin y Miller (2004) y Chen y col. (2008) utilizaron metodologías metaheurísticas basadas en búsqueda tabú, para concluir en la misma solución que Zamora y Grossmann (1998a). Petterson (2008) desarrolló un método de optimización global con una linealización por tramos del modelo Synheat (Yee y Grossmann, 1990) basado en la diferencia media geométrica de temperaturas (DMGT) permitiendo divisiones de corriente, pero restringiendo a mezclado isotérmico, consiguiendo un diseño que evaluado con la DMLT con costo de $\$ 80,962$ por año. Gupta y Ghosh (2010) determinan la mejor solución disponible hasta el momento utilizando un enfoque 
de aleatorización, obteniendo un diseño de red de intercambio de calor con costo de $\$ 80,379$ por año.

Tabla 5.7. Datos de corrientes y costos para el Caso de Estudio 5.2.

$\left.\begin{array}{ccccc}\hline \text { Corriente } & \begin{array}{c}\text { Temperatura de } \\ \text { suministro } \\ \mathrm{T}\left({ }^{\circ} \mathrm{C}\right)\end{array} & \begin{array}{c}\text { Temperatura } \\ \text { objetivo } \\ \mathrm{T}\left({ }^{\circ} \mathrm{C}\right)\end{array} & \begin{array}{c}\text { Flujo de } \\ \text { capacidad } \\ \text { calorífica } \\ \mathrm{F}\left(\mathrm{kW}{ }^{\circ} \mathrm{C}^{-1}\right)\end{array} & \begin{array}{c}\text { Coeficiente de } \\ \text { película }\end{array} \\ \mathrm{h}\left(\mathrm{kW} \mathrm{m}^{-2}{ }^{\circ} \mathrm{C}^{-1}\right)\end{array}\right)$

Costo de todos los equipos $\left(\$\right.$ año $\left.^{-1}\right)=7400+80\left[\right.$ Área $\left.\left(\mathrm{m}^{2}\right)\right]$

Costo de servicios de calentamiento $=110\left(\$ \mathrm{~kW}^{-1} \mathrm{año}^{-1}\right)$

Costo de servicios de enfriamiento $=10\left(\$ \mathrm{~kW}^{-1}\right.$ año $\left.^{-1}\right)$

\section{Elección de parámetros de entrada}

En la Tabla 5.8 se detallan algunos datos estimados para ciertos HRAT obtenidos el método de "Super-Targeting". El HRAT estimado como óptimo, es mostrado en la Figura 5.8 el cual presenta un valor de $33{ }^{\circ} \mathrm{C}$ para el Caso de Estudio 5.2.

Tabla 5.8. Valores estimados por el método de "Super-Targeting" para el Caso de Estudio 5.2.

\begin{tabular}{ccccc}
\hline $\begin{array}{c}\text { HRAT } \\
\left({ }^{\circ} \mathrm{C}\right)\end{array}$ & $\begin{array}{c}\text { Calor } \\
\text { recuperado } \\
\mathrm{q}_{\mathrm{rec}}(\mathrm{kW})\end{array}$ & $\begin{array}{c}\text { Servicios de } \\
\text { calentamiento } \\
\mathrm{qhu}(\mathrm{kW})\end{array}$ & $\begin{array}{c}\text { Servicios de } \\
\text { enfriamiento } \\
\mathrm{qcu}(\mathrm{kW})\end{array}$ & $\begin{array}{c}\text { CTA } \\
\text { estimado } \\
\left(\$ \text { año }^{-1}\right)\end{array}$ \\
\hline 10 & 276.05 & 106.45 & 85.58 & $82,681.83$ \\
20 & 256.44 & 126.06 & 105.19 & $79,103.90$ \\
32 & 232.91 & 149.59 & 128.73 & $78,158.91$ \\
$\mathbf{3 3}$ & $\mathbf{2 3 0 . 0 1}$ & $\mathbf{1 5 2 . 4 9}$ & $\mathbf{1 3 1 . 6 2}$ & $\mathbf{7 8 , 1 5 8 . 8 7}$ \\
34 & 227.12 & 155.38 & 134.51 & $78,163.22$ \\
40 & 209.75 & 172.75 & 151.88 & $78,377.20$ \\
\hline
\end{tabular}




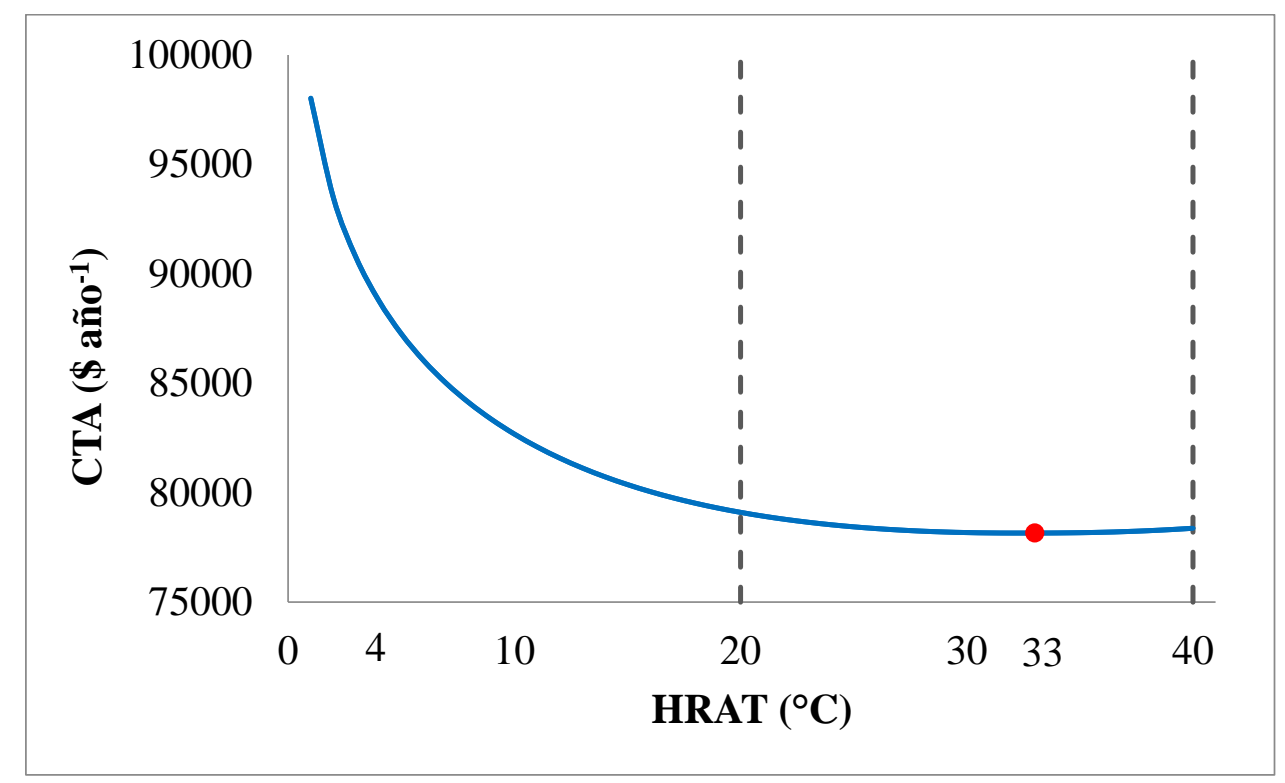

Figura 5.8. "Super-Targeting" y selección del intervalo de HRAT para la generación de topologías redundantes en el Caso de Estudio 5.2.

La generación de las diferentes topologías redundantes se elige en el intervalo que comprende desde un $\operatorname{HRAT}^{\mathrm{L}}=20^{\circ} \mathrm{C}$ hasta un $\operatorname{HRAT}^{\mathrm{U}}=40^{\circ} \mathrm{C}$ con un incremento en el HRAT en cada iteración de $t=1{ }^{\circ} \mathrm{C}$. Al aumentar el número de corrientes, las rutas de intercambio de calor entre éstas se incrementan por lo que se espera que los diseños iniciales presenten una mayor cantidad de equipos conforme el tamaño del problema crezca. Por esta razón para el Caso de Estudio 5.2 se elige incrementar a 500 inicializaciones por cada diseño base generado.

\section{Generación y optimización de topologías redundantes}

Para el Caso de Estudio 5.2 se procede a generar diseños base mediante los tres niveles de redundancia disponibles. En la Tabla 5.9 se muestran las estadísticas del problema abordado, donde es posible comprobar que el tiempo de cómputo se ha incrementado debido al número de inicializaciones y a que las topologías redundantes presentan un mayor número de equipos con relación con el Caso de Estudio 5.1. 
Tabla 5.9. Resultados del Caso de Estudio 5.2.

Nivel de redundancia Primer nivel Segundo nivel Tercer nivel

\begin{tabular}{lccc} 
Topologías redundantes generadas & 21 & 21 & 21 \\
Número de inicializaciones por diseño base & 500 & 500 & 500 \\
Soluciones Factibles determinadas & 10477 & 10495 & 10493 \\
Óptimos locales diferentes & 200 & 187 & 89 \\
Promedio de equipos en topologías redundantes & 20 & 13 & 10 \\
Promedio de equipos en diseños óptimos & 8 & 9 & 8 \\
Tiempo de CPU promedio por inicialización & $0.0164 \mathrm{~s}$ & $0.01282 \mathrm{~s}$ & $0.01113 \mathrm{~s}$ \\
Tiempo total de CPU & $2.87 \mathrm{~min}$ & $2.243 \mathrm{~min}$ & $1.948 \mathrm{~min}$ \\
\hline
\end{tabular}

En la Figura 5.9 se muestran los mejores óptimos locales para cada HRAT identificando a la solución con menor $\mathrm{CTA}_{\text {Paterson }}$ de $\$ 83,808$ por año con la aproximación de Paterson. Esta solución es determinada únicamente con un HRAT de $20{ }^{\circ} \mathrm{C}$ en el segundo y tercer nivel de redundancia, dicha solución presenta tres recuperadores de calor y requiere de calentamiento en la corriente fría $\mathrm{C} 2$, así como enfriamiento en las corrientes calientes $\mathrm{H} 1$ y H3 respectivamente.

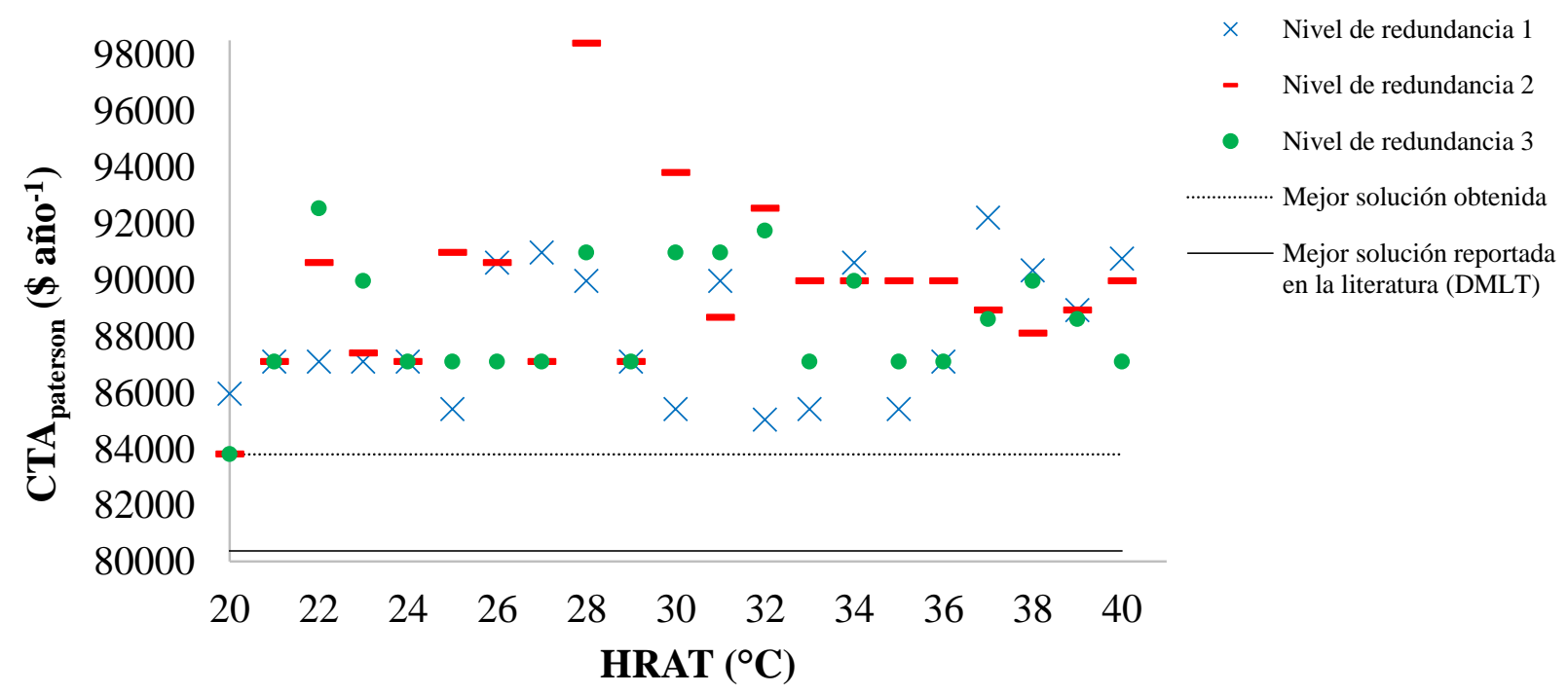

Figura 5.9. Óptimos locales de menor CTA Paterson para cada HRAT en el Caso de Estudio 5.2. 


\section{Generación y optimización de topologías redundantes restringiendo servicios auxiliares}

Al igual que en la Sección 5.2, se intenta mejorar las posibilidades de determinar soluciones de bajo costo prohibiendo servicios auxiliares para evitar que la metodología de optimización caiga soluciones poco convenientes. Utilizando la mejor solución obtenida, se decide prohibir los apareamientos de servicios auxiliares que no fueron requeridos, fijando $C C U_{2, l}=M \mathrm{y}$ $C H U_{1, l 1}=M$ lo que impide a la solución del modelo de transporte generar rutas entre las corrientes de proceso $\mathrm{H} 2$ y $\mathrm{C} 1$ con servicios de enfriamiento y calentamiento, respectivamente. En la Tabla 5.10 se presenta el resumen de resultados de la prueba realizada con servicios auxiliares limitados.

Tabla 5.10. Resultados del Caso de Estudio 5.2 prohibiendo servicios auxiliares a las corrientes de proceso $\mathrm{H} 2$ y $\mathrm{C} 1$.

\begin{tabular}{lccc} 
& Primer nivel & Segundo nivel & Tercer nivel \\
\hline Topologías redundantes generadas & 21 & 21 & 21 \\
Número de inicializaciones por diseño base & 500 & 500 & 500 \\
Soluciones factibles determinadas & 10347 & 10425 & 10486 \\
Óptimos locales diferentes & 156 & 212 & 40 \\
Promedio de equipos en topologías redundantes & 21 & 13 & 9 \\
Promedio de equipos en diseños óptimos & 8 & 8 & 7 \\
Tiempo de CPU promedio por inicialización & $0.0169 \mathrm{~s}$ & $0.01299 \mathrm{~s}$ & $0.01018 \mathrm{~s}$ \\
Tiempo total de CPU & $2.957 \mathrm{~min}$ & $2.273 \mathrm{~min}$ & $1.781 \mathrm{~min}$ \\
\hline
\end{tabular}

En la Figura 5.10 se muestran los resultados de la optimización de topologías que no incluyen los servicios auxiliares seleccionados. Como era de esperarse los óptimos locales con CTA $\mathrm{A}_{\text {Paterson }}$ de $\$ 83,808$ son obtenidos con mayor frecuencia al haber fijado que los servicios auxiliares favorezcan esta solución. Por otro lado, se puede reconocer una solución generada con un HRAT de $21{ }^{\circ} \mathrm{C}$ y el tercer nivel de redundancia que exhibe un menor costo en relación con la obtenida previamente sin limitar el uso de servicios auxiliares, la cual presenta un CTA Paterson $_{\text {de } \$ 80,840 .}$

La topología redundante que genera la solución con $\mathrm{CTA}_{\text {Paterson }}$ de $\$ 80,840$ por año se ilustra en la Figura 5.11, presentando 21 equipos de intercambio de calor. Para el modelo de PNL de esta estructura se utilizan 540 variables y 468 restricciones. 


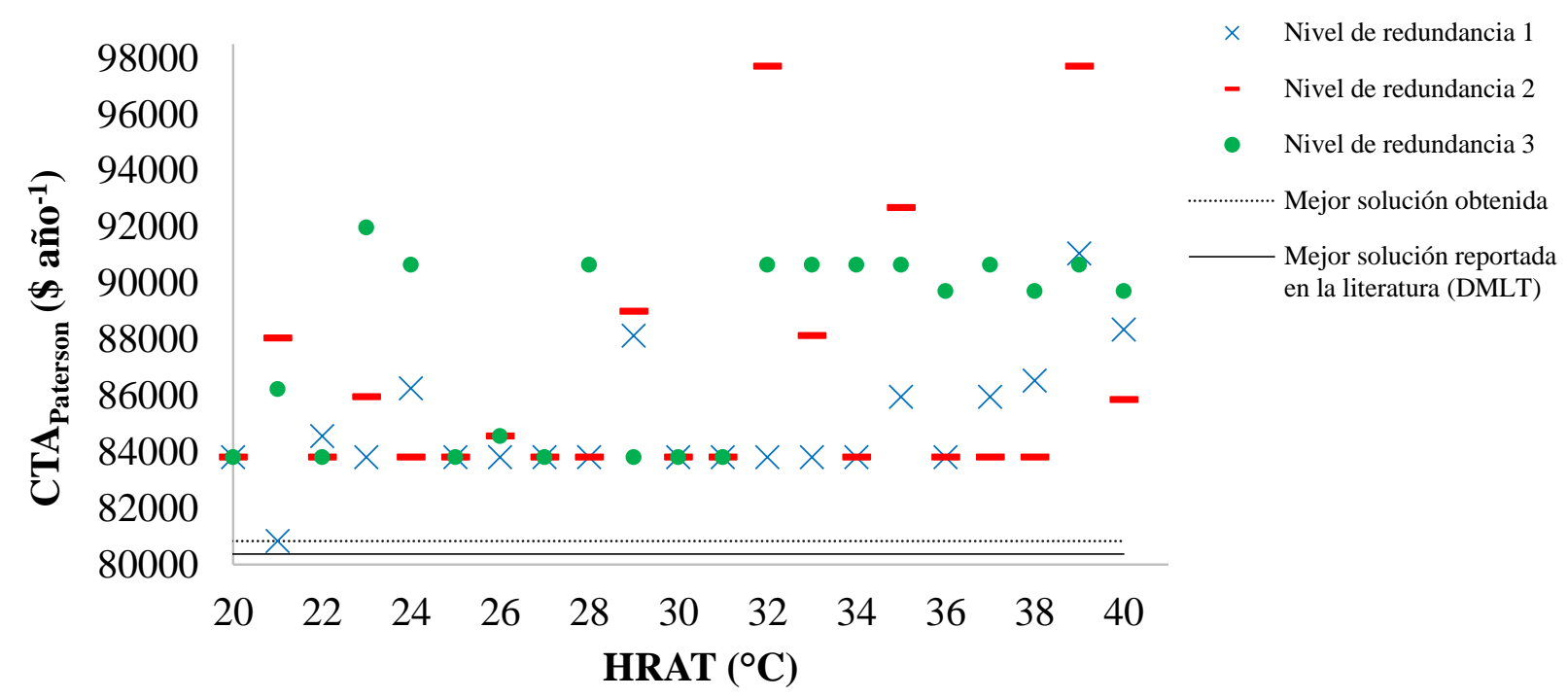

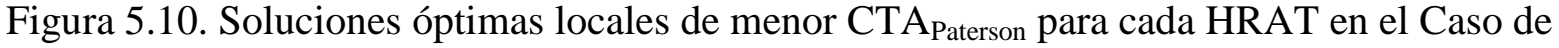

Estudio 5.2, prohibiendo los servicios auxiliares a las corrientes de proceso $\mathrm{H} 2$ y C1.

El diseño final se muestra en la Figura 5.12, en la cual únicamente permanecen 3 recuperadores y dos servicios auxiliares, dicha solución presenta un costo reevaluado con la DMTL de $\$ 80,847.71$ por año. Cabe mencionar que la misma estructura obtenida en este trabajo fue previamente determinada por Petterson (2008), quien afirma que la solución representa el óptimo global de las estructuras contenidas en la superestructura Synheat (Yee y Grossmann, 1990). La diferencia en el CTA de la red de intercambio de calor desarrollada en este trabajo y la de Petterson (2008) consiste en que la metodología aquí propuesta no requiere hacer la suposición de mezclado isotérmico.

La mejor solución reportada en la literatura desarrollada por Gupta y Ghosh (2010) tiene una estructura similar a la obtenida en este trabajo, difiriendo únicamente en que el calentador asociado a la corriente $\mathrm{C} 2$ se coloca en una sub-corriente paralelamente al intercambiador de calor con etiqueta número 13. Las estructuras con estas características no se incluyen en el modelo Synheat ni en las topologías redundantes generadas en este trabajo por lo que no es posible determinar dicha solución mediante estas formulaciones. 


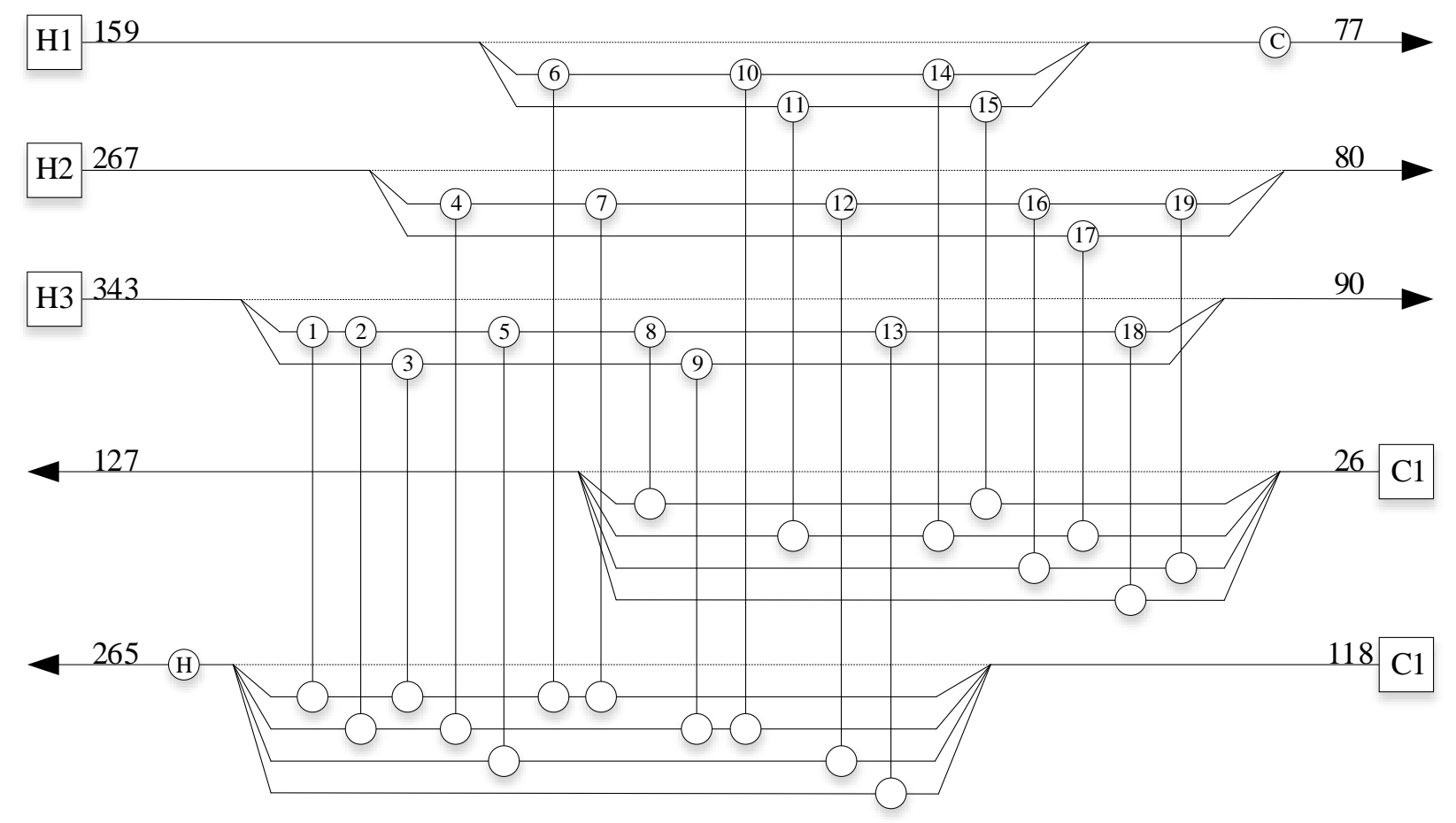

Figura 5.11. Topología redundante generada con un HRAT de $21^{\circ} \mathrm{C}$ y el primer nivel de redundancia para el Caso de Estudio 5.2.

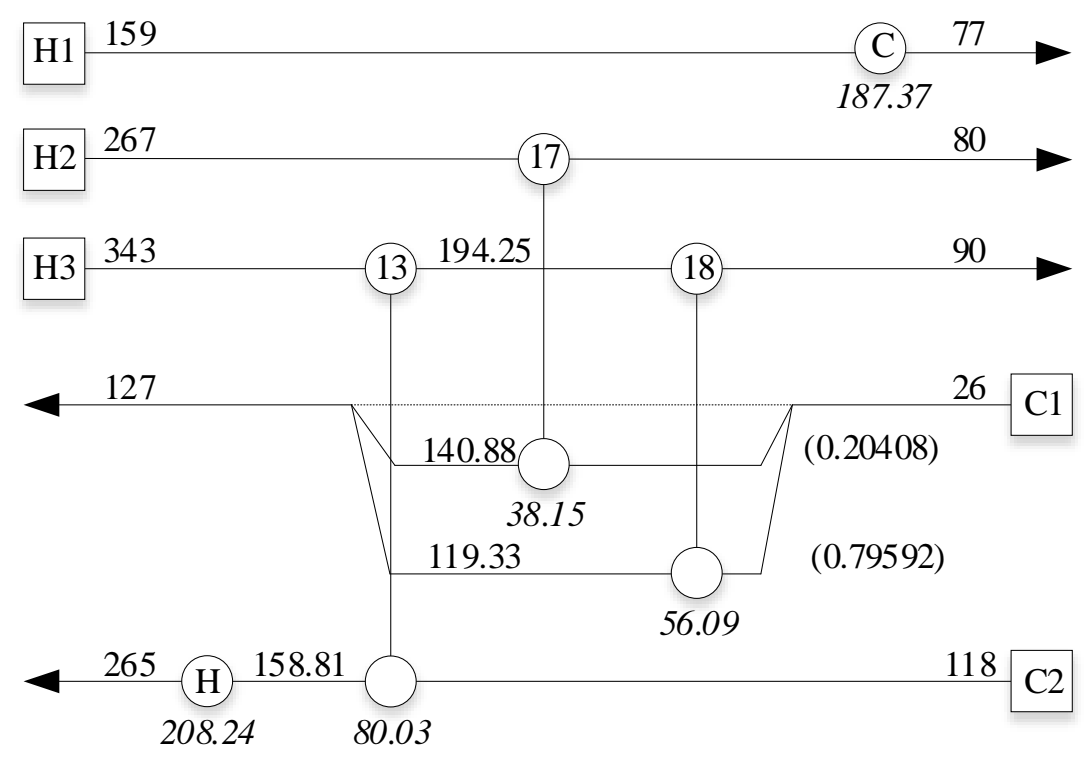

Figura 5.12. Mejor solución óptima local para el Caso de Estudio 5.2 con CTA de \$80,847 (temperaturas $\left({ }^{\circ} \mathrm{C}\right)$ en fuente normal, cargas térmicas $(\mathrm{kW})$ en cursivas y fracciones de flujo entre paréntesis). 


\section{Comparación de resultados}

En la Tabla 5.11 se detallan los resultados obtenidos por autores que han abordado el Caso de Estudio 5.2 comparados con la mejor solución obtenida en este trabajo.

Tabla 5.11. Comparación del Caso de Estudio 5.2 con soluciones reportadas en la literatura.

\begin{tabular}{|c|c|c|c|}
\hline Autor(es) & Metodología & Configuración $^{1}$ & $\begin{array}{c}\text { CTA Reportado } \\
\$ \text { año }^{-1}\end{array}$ \\
\hline $\begin{array}{l}\text { Zamora y Grossmann } \\
\text { (1997) }\end{array}$ & $\begin{array}{c}\text { Superestructura - } \\
\text { Optimización } \\
\text { Global }\end{array}$ & $3,1,1-0(0)$ & 83,400 \\
\hline Lin y Miller (2004) & Búsqueda Tabú & $3,1,1-0(0)$ & 83,400 \\
\hline Chen y col. (2008) & Búsqueda Tabú & $3,1,1-0(0)$ & 83,400 \\
\hline Rezaei y Shafiei (2008) & $\begin{array}{l}\text { Algoritmos } \\
\text { Genéticos }\end{array}$ & $3,1,1-0(0)$ & 82,151 \\
\hline Petterson $(2008)^{2}$ & $\begin{array}{c}\text { Superestructura - } \\
\text { Optimización } \\
\text { Global }\end{array}$ & $3,1,1-1(2)$ & 80,962 \\
\hline Gupta y Ghosh $(2010)^{2}$ & Búsqueda aleatoria & $3,1,1-1(2)$ & 80,379 \\
\hline Este trabajo & $\begin{array}{c}\text { Topologías } \\
\text { redundantes }\end{array}$ & $3,1,1-1(2)$ & $80,847.71$ \\
\hline
\end{tabular}

\subsection{Caso de Estudio 5.3}

El Caso de Estudio 5.3 es tomado de un trabajo de Trivedi (1998) el cual involucra cuatro corrientes de proceso calientes y tres corrientes de proceso frías, vapor y agua de enfriamiento. Los datos del problema se encuentran detallados en la Tabla 5.12.

Este problema es retomado por Huang y Karimi (2013) quienes desarrollaron dos metodologías basadas en superestructuras nuevas que incluyen características tales como permitir flujos cruzados, reapareamientos cíclicos, equipos en serie en sub-corrientes y múltiples servicios auxiliares que pueden ser intermedios. Estos autores utilizan la aproximación de Paterson para DMLT y determinan una solución con $\mathrm{CTA}_{\text {Paterson }}$ de $\$ 105,403$, comparando su enfoque 
simultáneo con la metodología de hiperestructura desarrollada por Ciric y Floudas (1991) quienes reportan una solución de $\$ 114,460$ por año.

Tabla 5.12. Datos de corrientes y costos para el Caso de Estudio 5.3.

\begin{tabular}{|c|c|c|c|c|}
\hline Corriente & $\begin{array}{l}\text { Temperatura de } \\
\text { suministro } \\
\mathrm{T}\left({ }^{\circ} \mathrm{C}\right)\end{array}$ & $\begin{array}{l}\text { Temperatura } \\
\text { objetivo } \\
\mathrm{T}\left({ }^{\circ} \mathrm{C}\right)\end{array}$ & $\begin{array}{c}\text { Flujo de } \\
\text { capacidad } \\
\text { calorífica } \\
\mathrm{F}\left(\mathrm{kW}{ }^{\circ} \mathrm{C}^{-1}\right)\end{array}$ & $\begin{array}{l}\text { Coeficiente de } \\
\text { película } \\
\mathrm{h}\left(\mathrm{kW} \mathrm{m}^{-2}{ }^{\circ} \mathrm{C}^{-1}\right)\end{array}$ \\
\hline H1 & 160 & 110 & 7.032 & 1.6 \\
\hline $\mathrm{H} 2$ & 249 & 138 & 8.440 & 1.6 \\
\hline H3 & 227 & 106 & 11.816 & 1.6 \\
\hline $\mathrm{H} 4$ & 271 & 146 & 7.000 & 1.6 \\
\hline $\mathrm{C} 1$ & 96 & 160 & 9.144 & 1.6 \\
\hline $\mathrm{C} 2$ & 116 & 217 & 7.296 & 1.6 \\
\hline C3 & 140 & 250 & 18.00 & 1.6 \\
\hline $\mathrm{HU}$ & 300 & 300 & - & 1.6 \\
\hline $\mathrm{CU}$ & 70 & 90 & - & 1.6 \\
\hline \multicolumn{5}{|c|}{$\begin{array}{l}\text { Costo de todos los equipos }\left(\$ \text { año }^{-1}\right)=1300\left[\text { Área }\left(\mathrm{m}^{2}\right)\right]^{0.6} \\
\text { Costo de servicios de calentamiento }=80\left(\$ \mathrm{~kW}^{-1} \mathrm{año}^{-1}\right) \\
\text { Costo de servicios de enfriamiento }=20\left(\$ \mathrm{~kW}^{-1} \mathrm{año}^{-1}\right)\end{array}$} \\
\hline
\end{tabular}

\section{Elección de parámetros de entrada}

En la Tabla 5.13 se muestran las metas determinadas por el método de "Super-Targeting" para algunos valores de HRAT. En la Figura 5.13 se ilustra la curva del CTA estimado en función del HRAT identificando el valor óptimo en $13{ }^{\circ} \mathrm{C}$. Se elige generar las topologías redundantes desde un $\operatorname{HRAT}^{\mathrm{L}}=12{ }^{\circ} \mathrm{C}$ hasta $\mathrm{HRAT}^{\mathrm{U}}=14{ }^{\circ} \mathrm{C}$ con un incremento de $t=0.1{ }^{\circ} \mathrm{C}$. Asignando 1000 inicializaciones a cada diseño base. A diferencia de las Secciones 5.2 y 5.3 el tamaño de paso se selecciona como número fraccional en lugar de un incremento unitario para el HRAT. Se elige de esta manera debido a que, al aumento en el número de corrientes de proceso del problema, otorga una mayor sensibilidad a pequeñas variaciones en el HRAT en las distribuciones de cargas térmicas que se producen de la solución del modelo de transporte, esta característica no tenía un fuerte impacto en los casos de estudio anteriores pues los incrementos menores a uno generaban, casi en su totalidad, las mismas topologías redundantes que los HRAT enteros contiguos. 
Tabla 5.13. Valores estimados por el método de "Super-Targeting" para el Caso de Estudio 5.3.

\begin{tabular}{ccccc}
\hline $\begin{array}{c}\text { HRAT } \\
\left({ }^{\circ} \mathrm{C}\right)\end{array}$ & $\begin{array}{c}\text { Calor } \\
\text { recuperado } \\
\text { qrec }(\mathrm{kW})\end{array}$ & $\begin{array}{c}\text { Servicios de } \\
\text { calentamiento } \\
\mathrm{qhu}(\mathrm{kW})\end{array}$ & $\begin{array}{c}\text { Servicios de } \\
\text { enfriamiento } \\
\mathrm{qcu}(\mathrm{kW})\end{array}$ & $\begin{array}{c}\text { CTA } \\
\text { estimado } \\
\left(\$ \mathrm{año}^{-1}\right)\end{array}$ \\
\hline 1 & 3302.11 & 0 & 291.06 & $113,316.90$ \\
10 & 3201.79 & 100.32 & 391.384 & $103,237.68$ \\
12 & 3151.2 & 150.91 & 441.976 & $102,049.24$ \\
$\mathbf{1 3}$ & $\mathbf{3 1 2 5 . 9 0}$ & $\mathbf{1 7 6 . 2 1}$ & $\mathbf{4 6 7 . 2 7 2}$ & $\mathbf{1 0 1 , 9 9 7 . 2 1}$ \\
14 & 3100.61 & 201.50 & 492.568 & $102,220.13$ \\
20 & 2897.27 & 404.84 & 695.904 & $110,096.72$ \\
\hline
\end{tabular}

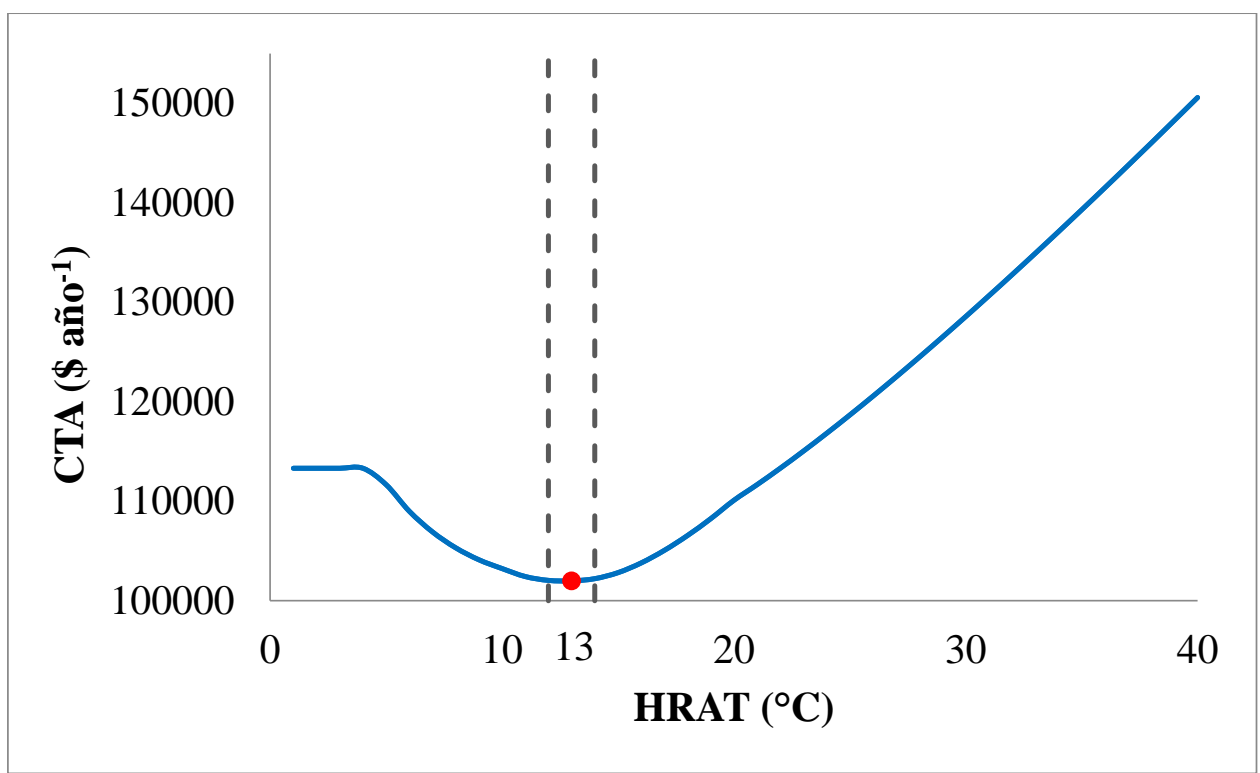

Figura 5.13. "Super-Targeting” y selección del intervalo de HRAT para la generación de topologías redundantes para el Caso de Estudio 5.3.

Aunque bien se podrían explorar una cantidad de topologías redundantes generadas con HRAT enteros más alejados del óptimo, se mantiene la premisa de que los diseños base construidos cercanos al HRAT óptimo tienen una buena posibilidad de generar estructuras de redes con las características adecuadas para la optimización. Se pretende que los diseños construidos posean valores de HRAT cercanos al óptimo, tratando de que éstos guarden alguna relación con los valores estimados por el método de "Super-Targeting".

Si bien, como se comentó en la Sección 5.2, pudiera darse el caso de que la mejor solución se determine en un HRAT no necesariamente cercano al óptimo, alejarse de este valor implica 
cierto riesgo; el HRAT proporciona el desplazamiento relativo de las corrientes de proceso en la tabla de calor, que establece las rutas de intercambio de calor en el planteamiento del modelo de transporte, por lo que un HRAT alejado del óptimo pudiera comprometer la integración térmica entre corrientes de proceso, conduciendo a topologías redundantes con un uso inadecuado de servicios auxiliares.

Por las razones mencionadas, la elección del incremento en el HRAT se propone como un número fraccional permitiendo evaluar múltiples topologías redundantes diferentes, con un HRAT cercano al estimado como óptimo.

\section{Generación y optimización de topologías redundantes}

En la Tabla 5.14 se describen resultados significativos al optimizar las redes de intercambio de calor generadas. Para este caso de estudio puede observarse que tanto el segundo y tercer nivel de redundancia generan una cantidad de equipos muy por debajo de las topologías desarrolladas mediante el primer nivel.

Tabla 5.14. Resultados del Caso de Estudio 5.3.

\begin{tabular}{lccc} 
& Primer nivel & Segundo nivel & Tercer nivel \\
\cline { 2 - 4 } & 21 & 21 & 21 \\
Topologías redundantes generadas & 1000 & 1000 & 1000 \\
Número de inicializaciones por diseño base & 18689 & 19177 & 18835 \\
Soluciones factibles determinadas & 949 & 1273 & 933 \\
Óptimos locales diferentes & 42 & 24 & 16 \\
Promedio de equipos en topologías redundantes & 9 & 9 & 8 \\
Promedio de equipos en diseños óptimos & $0.03116 \mathrm{~s}$ & $0.018 \mathrm{~s}$ & $0.01788 \mathrm{~s}$ \\
Tiempo de CPU promedio por inicialización & $10.907 \mathrm{~min}$ & $6.301 \mathrm{~min}$ & $6.258 \mathrm{~min}$ \\
Tiempo total de CPU & & & \\
\hline
\end{tabular}

La generación y optimización de topologías redundantes para los parámetros seleccionados se

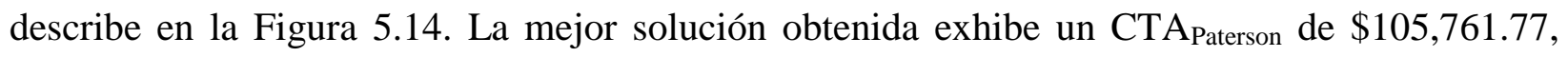
generada con un HRAT de $14{ }^{\circ} \mathrm{C}$ en el segundo y tercer nivel de redundancia. La red de intercambio de calor presenta cinco recuperadores de calor, un calentador en la corriente C3 y enfriadores en las corrientes $\mathrm{H} 1, \mathrm{H} 2$ y H3. El diseño de red determinado sin restringir servicios 
auxiliares presenta una diferencia únicamente un $0.34 \%$ mayor que la solución reportada por Huang y Karimi (2013), utilizando en ambas soluciones la aproximación de Paterson para la DMLT. La red de intercambio de calor presenta un costo que puede considerarse competitivo, sin embargo, se desea explotar la capacidad de la metodología utilizando la estrategia de prohibir servicios auxiliares con el fin de encontrar alguna solución con el menor costo posible.

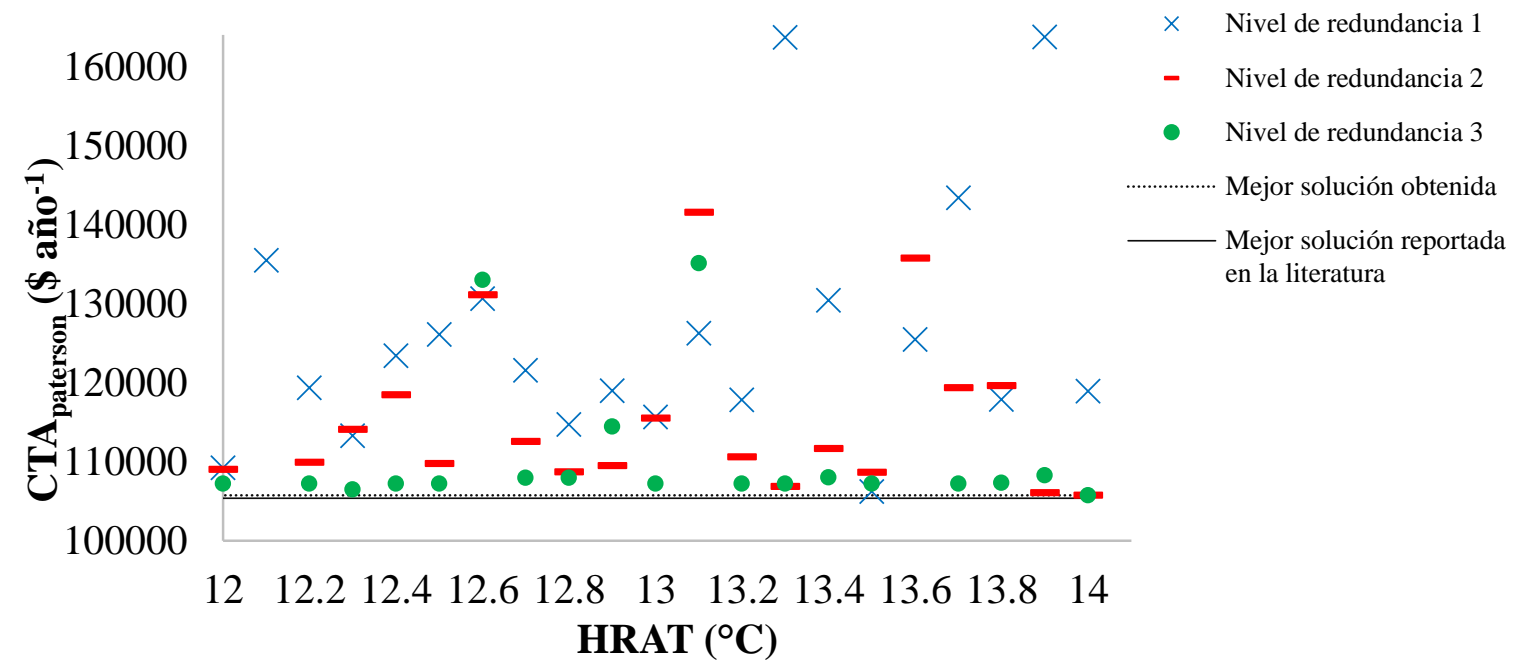

Figura 5.14. Óptimos locales de menor CTA $\mathrm{A}_{\text {Paterson }}$ para cada HRAT en el Caso de Estudio 5.3.

\section{Generación y optimización de topologías redundantes restringiendo servicios auxiliares}

La limitación de servicios auxiliares ha permitido obtener soluciones de bajo costo en comparación que en topologías redundantes cuya asignación de servicios auxiliares es determinada por la solución del modelo de transporte. Mediante este concepto se procede a prohibir los servicios auxiliares que no fueron requeridos en la mejor solución obtenida hasta el momento para el Caso de Estudio 5.3. Se fijan costos de transporte altos a los apareamientos de las corrientes $\mathrm{H} 4, \mathrm{C} 1$ y $\mathrm{C} 2$ con servicios auxiliares, de modo que $C C U_{4, l}=M, C H U_{1, l 1}=M$ y $C H U_{2, l 1}=M$. En la Tabla 5.15 se muestran los resultados estadísticos del Caso de Estudio 5.3. En la Figura 5.15 se detallan los resultados de la optimización de topologías redundantes prohibiendo el uso de servicios auxiliares, generadas mediante los tres niveles de redundancia. En este caso, a pesar de que se favorecieron las condiciones bajo las cuales se obtuvo la mejor solución determinada anteriormente sin prohibir servicios auxiliares, la mejor solución de esta 
prueba asciende a un $\mathrm{CTA}_{\text {Paterson }}$ de $\$ 105,828.36$, generada con un HRAT de $12.5{ }^{\circ} \mathrm{C}$ y el segundo nivel de redundancia, siendo $\$ 67$ más cara que la previamente obtenida con CTA $\mathrm{A}_{\text {Paterson }}$ de $\$ 105,761.77$. Esto resalta los riesgos que pudiera implicar realizar un número de iteraciones insuficiente para asegurarse de que óptimos locales de calidad no han sido omitidos.

Tabla 5.15. Resultados del Caso de Estudio 5.3 prohibiendo servicios auxiliares a las corrientes de proceso $\mathrm{H} 4, \mathrm{C} 1$ y $\mathrm{C} 2$.

\begin{tabular}{lccc} 
& Primer nivel & Segundo nivel & Tercer nivel \\
\cline { 2 - 4 } & 21 & 21 & 21 \\
Topologías redundantes generadas & 1000 & 1000 & 1000 \\
Súmero de inicializaciones por diseño base & 19206 & 18196 & 19668 \\
Soluciones factibles determinadas & 762 & 1079 & 686 \\
Óptimos locales diferentes & 42 & 25 & 16 \\
Promedio de equipos en topologías redundantes & 9 & 9 & 8 \\
Promedio de equipos en diseños óptimos & $0.02989 \mathrm{~s}$ & $0.01605 \mathrm{~s}$ & $0.01701 \mathrm{~s}$ \\
Tiempo de CPU promedio por inicialización & $10.46 \mathrm{~min}$ & $5.618 \mathrm{~min}$ & $5.955 \mathrm{~min}$ \\
Tiempo total de CPU & & & \\
\hline
\end{tabular}

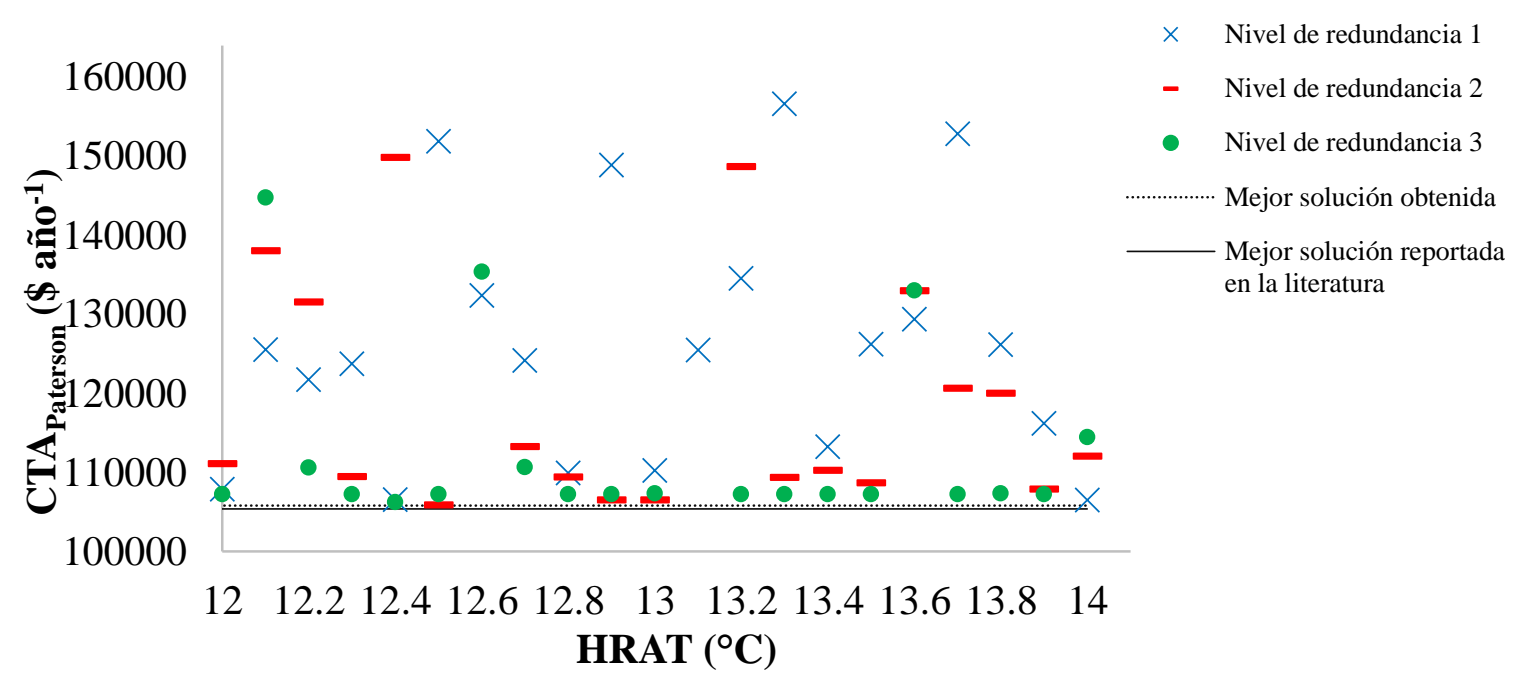

Figura 5.15. Soluciones óptimas locales de menor CTA Paterson para cada HRAT en el Caso de

Estudio 5.3, prohibiendo los servicios auxiliares a las corrientes de proceso $\mathrm{H} 4, \mathrm{C} 1$ y $\mathrm{C} 2$.

Una alternativa para determinar nuevas soluciones consiste en cambiar la elección de servicios auxiliares prohibidos para generar topologías redundantes con diferentes características como se realizó en la Sección 5.2, sin embargo, se desea explotar las capacidades de automatización de la metodología desarrollada, sin dar pie a incertidumbres causadas por tomas de decisiones. Antes 
de que el diseñador decida darse a la tarea de realizar numerosas pruebas individuales mediante la limitación de diferentes servicios auxiliares, se propone diversificar la generación de topologías, con el fin de explorar una mayor cantidad de diseños iniciales, e intensificar el número de inicializaciones del enfoque de optimización, buscando disminuir las probabilidades de omitir óptimos locales de calidad. Se plantea realizar la generación de topologías redundantes en el mismo intervalo de parámetros HRAT, pero disminuyendo el tamaño de paso a $t=0.01{ }^{\circ} \mathrm{C}$. Por otro lado, como las tipologías redundantes generadas en el Caso de Estudio 5.3 tienen un número considerablemente mayor que las topologías que representaban a los casos de estudio anteriores, se incrementa el número de inicializaciones a 2000 por cada diseño base. La generación de topologías redundantes se formula manteniendo prohibidos los servicios en las corrientes de proceso $\mathrm{H} 4, \mathrm{C} 1$ y $\mathrm{C} 2$.

La propuesta de diversificación e intensificación supone un consumo de recursos computacionales considerablemente mayor a las pruebas reportadas previamente en esta sección, por este motivo dicha prueba se ejecuta únicamente en el primer nivel de redundancia. Se elige el mayor tamaño disponible debido a que, al incrementar el número de inicializaciones, las topologías de mayor tamaño pueden verse beneficiadas al presentar una mayor cantidad de equipos, tal y como se argumenta en la Sección 3.5. Estos diseños tienen una alta probabilidad de contener una diversidad mayor de óptimos locales en comparación con aquellas que presentan un número reducido de equipos.

Tabla 5.16. Resultados del Caso de Estudio 5.3 prohibiendo servicios auxiliares a las corrientes de proceso e intensificando la búsqueda.

\begin{tabular}{lc}
\cline { 2 - 2 } & Primer nivel \\
\hline Topologías redundantes generadas & 201 \\
Número de inicializaciones por diseño base & 2000 \\
Soluciones factibles determinadas & 356906 \\
Óptimos locales diferentes & 5945 \\
Promedio de equipos en topologías redundantes & 43 \\
Promedio de equipos en diseños óptimos & 9 \\
Tiempo de CPU promedio por inicialización & $0.02905 \mathrm{~s}$ \\
Tiempo total de CPU & $3.244 \mathrm{hrs}$ \\
\hline
\end{tabular}


En la Tabla 5.16 se muestra el resumen de resultados para la prueba realizada. Como se anticipó, el tiempo de cómputo se incrementa considerablemente, por lo que este recurso debe considerarse como una opción solo si se desean llevar al límite las capacidades de la metodología propuesta en este trabajo. Por otro lado, la generación de una mayor cantidad de topologías redundantes junto con una búsqueda más exhaustiva de óptimos locales produce un incremento importante en la diversidad de soluciones óptimas diferentes.

En la Figura 5.16 se detallan los óptimos locales de menor costo para la prueba realizada en cada HRAT. El óptimo local determinado en la primera prueba de esta sección con CTA $\mathrm{Paterson}_{\text {de }}$ $\$ 105,761.77$ se determinó una sola ocasión en el diseño generado con un HRAT de 13.38, la

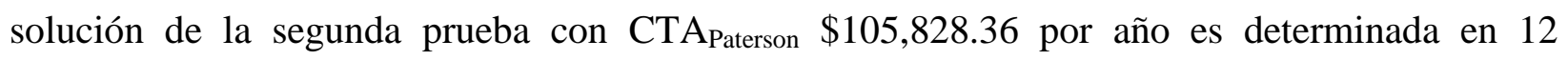
ocasiones. La inversión en recursos computacionales se ve recompensada con una nueva solución que presenta un menor costo con respecto a las soluciones obtenidas en pruebas anteriores. La mejor solución determinada presenta un CTA Paterson de $\$ 105,460.52$, la cual es revelada en dos ocasiones mediante el HRAT de $12.96{ }^{\circ} \mathrm{C}$ y el HRAT de $13.6{ }^{\circ} \mathrm{C}$. Los resultados demuestran que, a cambio de un alto costo computacional, es posible generar y optimizar un mayor número de topologías redundantes, lo que se asocia al incremento en las posibilidades de determinar soluciones con costo competitivo que pudieran surgir con poca frecuencia.

La topología redundante generada con un HRAT de $12.96{ }^{\circ} \mathrm{C}$ que contiene a la mejor solución determinada, se muestra en la Figura 5.17. Esta estructura presenta 39 recuperadores de calor, dos calentadores y dos enfriadores. El planteamiento del modelo de PNL para la optimización de la red redundante se formula con 1424 variables y 1284 restricciones. El diseño simplificado con

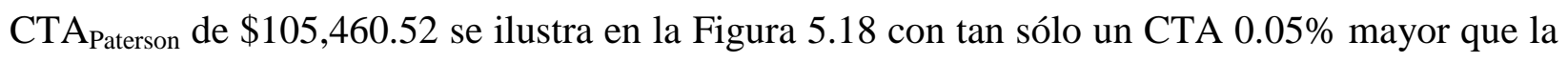
mejor solución reportada en la literatura desarrollada por Huang y Karimi (2013) utilizando la aproximación de Paterson. La reevaluación de la red de intercambio de calor obtenida en este trabajo presenta un costo final reevaluado con la DMLT de $\$ 105,481.12$ por año. Cabe destacar que ambas soluciones presentan el mismo número de equipos y una estructura con asignación similar en los valores de las variables de decisión, diferenciándose únicamente en que el calentador se coloca en serie sobre la primer sub corriente de C3, a la salida del intercambiador de calor con la etiqueta número 6. 


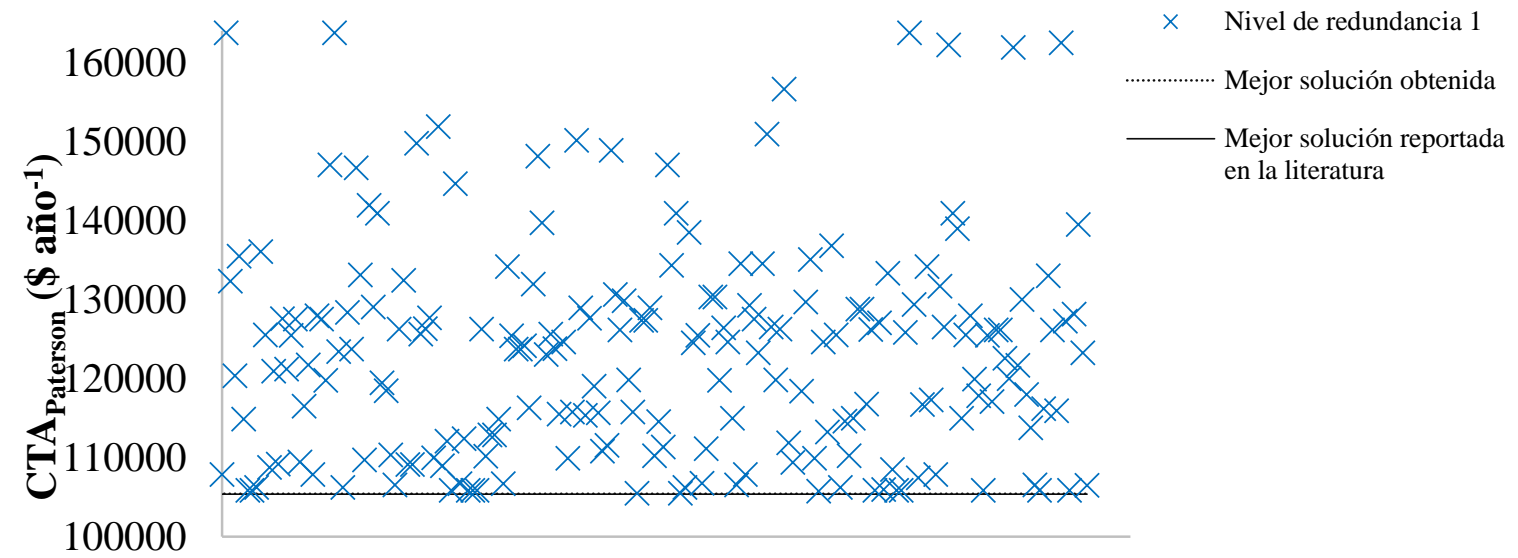

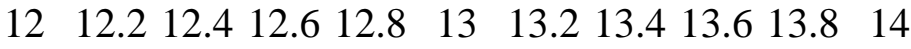 HRAT $\left({ }^{\circ} \mathrm{C}\right)$}

Figura 5.16. Búsqueda intensiva de soluciones óptimas locales para cada HRAT en el Caso de Estudio 5.3, prohibiendo los servicios auxiliares a las corrientes de proceso $\mathrm{H} 1, \mathrm{C} 2$ y C3.

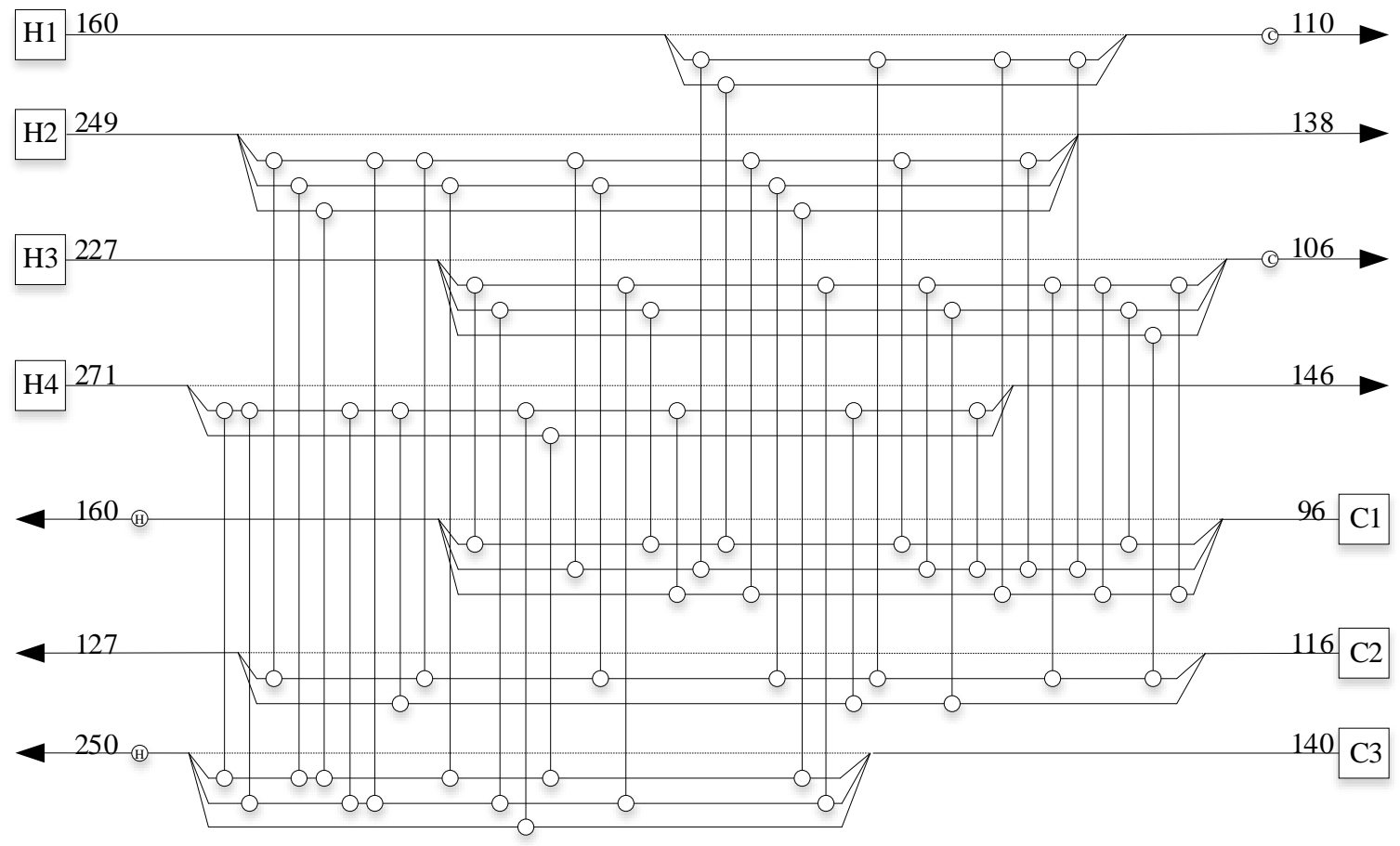

Figura 5.17. Topología redundante generada con un HRAT de $12.96{ }^{\circ} \mathrm{C}$ y el tercer nivel de redundancia para el Caso de Estudio 5.3.

Aunque la selección de servicios auxiliares intermedios no está considerada en la implementación de este trabajo, se puede señalar que el tiempo de cómputo es considerablemente menor en comparación con el de la metodología desarrollada por Huang y Karimi (2013), la cual 
requiere un total de 48 horas de CPU para obtener la solución reportada con CTA Paterson de \$105,403 siendo únicamente \$57 por año más económica que la determinada en este trabajo.

Una característica destacable de la metodología propuesta se se puede apreciar en la Figura 5.18 sobre la primer sub-corriente de C3, donde se colocan dos intercambiadores de calor sobre un mismo ramal de una sub-corriente de proceso, dichos elementos no están incluidos en algunas de las metodologías de síntesis basadas en superestructuras clásicas como Synheat (Yee y Grossmann, 1990) o la hiperestructura de Ciric y Floudas (1990).

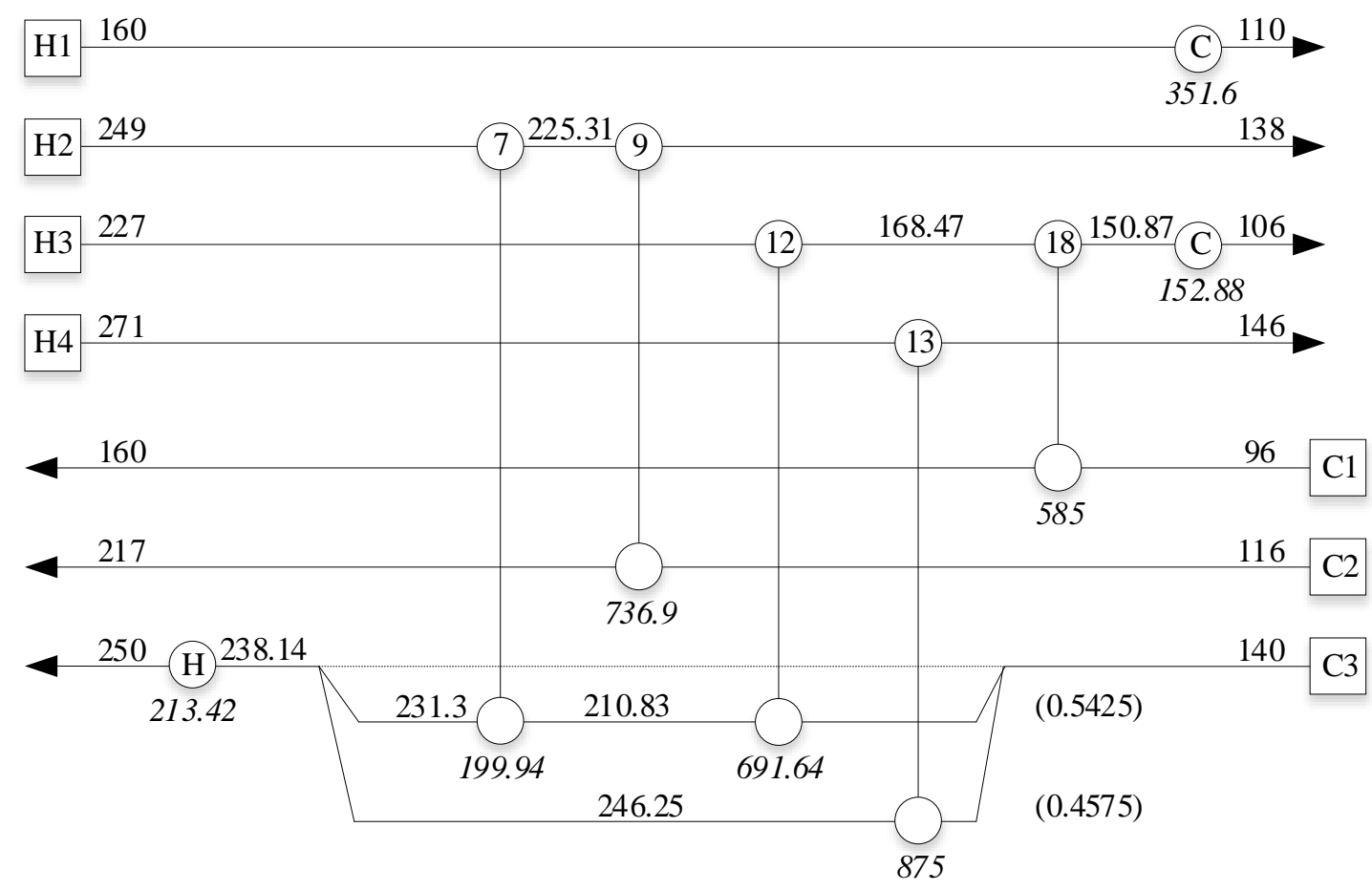

Figura 5.18. Mejor solución óptima local para el Caso de Estudio 5.3 con CTA de \$105,481.12 (temperaturas $\left({ }^{\circ} \mathrm{C}\right)$ en fuente normal, cargas térmicas $(\mathrm{kW})$ en cursivas y fracciones de flujo entre paréntesis).

\section{Comparación de resultados}

En la Tabla 5.17 se detallan los resultados obtenidos por autores que han abordado el Caso de Estudio 5.3 comparados con la mejor solución obtenida en este trabajo. 
Tabla 5.17. Comparación del Caso de Estudio 5.3 con soluciones reportadas en la literatura.

\begin{tabular}{lccc}
\hline \multicolumn{1}{c}{ Autor(es) } & Metodología & Configuración $^{1}$ & $\begin{array}{c}\text { CTA Reportado } \\
\$ \text { año }^{-1}\end{array}$ \\
\hline Ciric y Foudas (1991) & $\begin{array}{c}\text { Hiperestructura } \\
\text { Algoritmos genéticos/ }\end{array}$ & - & 114,460 \\
Luo y col. (2004) & Recocido simulado & $6,1,2-2(6)$ & 107.124 \\
Huang y Karimi (2013) & Superestructuras & $5,1,2-1(2)$ & $105,403 *$ \\
Este trabajo & Topologías redundantes & $\mathbf{5 , 1 , 2 - 1 ( 2 )}$ & $\mathbf{1 0 5 , 4 8 1 . 1 2}$ \\
\hline $\begin{array}{l}{ }^{1} \text { Recuperadores,Calentadores,Enfriadores-No. de Divisores de corrientes(No. de Sub-corrientes). } \\
{ }^{2} \text { Solución revisada en el Apéndice A } \\
{ }^{*} \text { CTA reportado con la aproximación de Paterson }\end{array}$ &
\end{tabular}

\subsection{Caso de Estudio 5.4}

El Caso de Estudio 5.4 es el problema 8SP1 extraído del trabajo de Grossmann y Sargent (1978) que consiste en la integración térmica de cuatro corrientes calientes y cuatro corrientes frías. Los datos del problema junto con la información de costos se muestran en la Tabla 5.18.

Tabla 5.18. Datos de corrientes y costos para el Caso de Estudio 5.4.

\begin{tabular}{ccccc}
\hline Corriente & $\begin{array}{c}\text { Temperatura de } \\
\text { suministro } \\
\mathrm{T}\left({ }^{\circ} \mathrm{F}\right)\end{array}$ & $\begin{array}{c}\text { Temperatura } \\
\text { objetivo } \\
\mathrm{T}\left({ }^{\circ} \mathrm{F}\right)\end{array}$ & $\begin{array}{c}\text { Flujo de } \\
\text { capacidad } \\
\text { calorífica } \\
\mathrm{F}\left(\mathrm{kBtu} \mathrm{h}^{-1}{ }^{\circ} \mathrm{C}^{-1}\right)\end{array}$ & $\begin{array}{c}\text { Coeficiente de película } \\
\mathrm{h}\left(\mathrm{kBtu}\left(\mathrm{h} \mathrm{ft}{ }^{\circ}{ }^{\circ} \mathrm{F}\right)^{-1}\right)\end{array}$ \\
\hline $\mathrm{H} 1$ & 470 & 320 & 22.4 & 0.30 \\
$\mathrm{H} 2$ & 450 & 240 & 17.5 & 0.30 \\
$\mathrm{H} 3$ & 370 & 150 & 28.5 & 0.30 \\
$\mathrm{H} 4$ & 310 & 200 & 20.1 & 0.30 \\
$\mathrm{C} 1$ & 200 & 420 & 16.8 & 0.30 \\
$\mathrm{C} 2$ & 150 & 400 & 23.2 & 0.30 \\
$\mathrm{C} 3$ & 185 & 330 & 35.1 & 0.30 \\
$\mathrm{C} 4$ & 140 & 300 & 17.25 & 0.30 \\
$\mathrm{HU}$ & 456 & 456 & - & 0.30 \\
$\mathrm{CU}$ & 100 & 180 & - & 0.30 \\
\hline
\end{tabular}

Costo de todos los equipos $\left(\$\right.$ año $\left.^{-1}\right)=35\left[\text { Área }\left(\mathrm{ft}^{2}\right)\right]^{0.6}$

Costo de servicios de calentamiento $=11.05\left(\$(\mathrm{kBtu} / \mathrm{h})^{-1} \mathrm{anno}^{-1}\right)$

Costo de servicios de enfriamiento $=5.31\left(\$(\mathrm{kBtu} / \mathrm{h})^{-1} \mathrm{año}^{-1}\right)$ 
Este caso de estudio es el único que se aborda con datos en el Sistema Inglés de unidades, debido a que los autores que lo han retomado han decidido mantener los datos en el sistema original. Grossmann y Sargent (1978) resolvieron este problema con un enfoque de optimización con dos etapas formulando un algoritmo que permite definir una configuración óptima y usar un modelo de PNL para optimizar las variables de la red, los autores reportan una solución con CTA de $\$ 31,890$. La mejor solución disponible en la literatura de $\$ 29,438$ por año fue determinada por Luo y col. (2004) usando un método híbrido que combina algoritmos genéticos con recocido simulado.

\section{Elección de parámetros de entrada}

Para este caso de estudio, la técnica de "Super-Targeting" para la elección del intervalo de parámetros HRAT sobre los cuales se construyen las topologías redundantes juega un papel sumamente importante. En la Figura 5.19 se describen las metas del CTA en función del HRAT, sin embargo, se puede observar una región plana desde un HRAT de $1{ }^{\circ} \mathrm{F}$ hasta el HRAT de 6 ${ }^{\circ} \mathrm{F}$, esto significa que la diferencia de temperaturas se encuentra sobre el "umbral" (Linnhoff y Hindmarsh, 1983) donde únicamente servicios de calentamiento o enfriamiento son requeridos, pero no ambos; para este problema en particular son requeridos únicamente servicios de calentamiento como puede apreciarse en la Tabla 5.19. En un problema de umbral, a pesar de incrementar el HRAT, las metas de energía permanecen constantes por lo que no se distingue un punto de pliegue, haciendo imposible determinar un único HRAT óptimo. No obstante, es posible elegir la sección de umbral como parámetro de diseño. Otra observación relevante es que al sobrepasar el punto de umbral incrementando ligeramente el HRAT por encima de $6{ }^{\circ} \mathrm{F}$, no es posible determinar las metas de costos mediante la metodología de "Super-Targeting".

Tabla 5.19. Valores estimados por el método de "Super-Targeting" para el Caso de Estudio 5.4.

\begin{tabular}{|c|c|c|c|c|}
\hline $\begin{array}{l}\text { HRAT } \\
\left({ }^{\circ} \mathrm{F}\right)\end{array}$ & $\begin{array}{c}\text { Calor } \\
\text { recuperado } \\
\mathrm{q}_{\text {rec }}\left(\mathrm{kBtu} \mathrm{h}^{-1}\right)\end{array}$ & $\begin{array}{l}\text { Servicios de } \\
\text { calentamiento } \\
\text { qhu }\left(\mathrm{kBtu} \mathrm{h}^{-1}\right)\end{array}$ & $\begin{array}{l}\text { Servicios de } \\
\text { enfriamiento } \\
\mathrm{qcu}\left(\mathrm{kBtu} \mathrm{h}^{-1}\right)\end{array}$ & $\begin{array}{c}\text { CTA } \\
\text { estimado } \\
\left(\$ \text { año }^{-1}\right)\end{array}$ \\
\hline 1 & 15516 & 1829.5 & $\mathbf{0}$ & $28,757.98$ \\
\hline 6 & 15516 & 1829.5 & $\mathbf{0}$ & $28,757.98$ \\
\hline
\end{tabular}


Tomando los valores de umbral se consideran los parámetros de entrada en el intervalo $\mathrm{HRAT}^{\mathrm{L}}=$ $1{ }^{\circ} \mathrm{F}$ hasta $\mathrm{HRAT}^{\mathrm{U}}=6^{\circ} \mathrm{F}$ para la construcción de topologías redundantes con un tamaño de paso $t=0.1^{\circ} \mathrm{F}$. Asignando 1000 inicializaciones a cada diseño base.

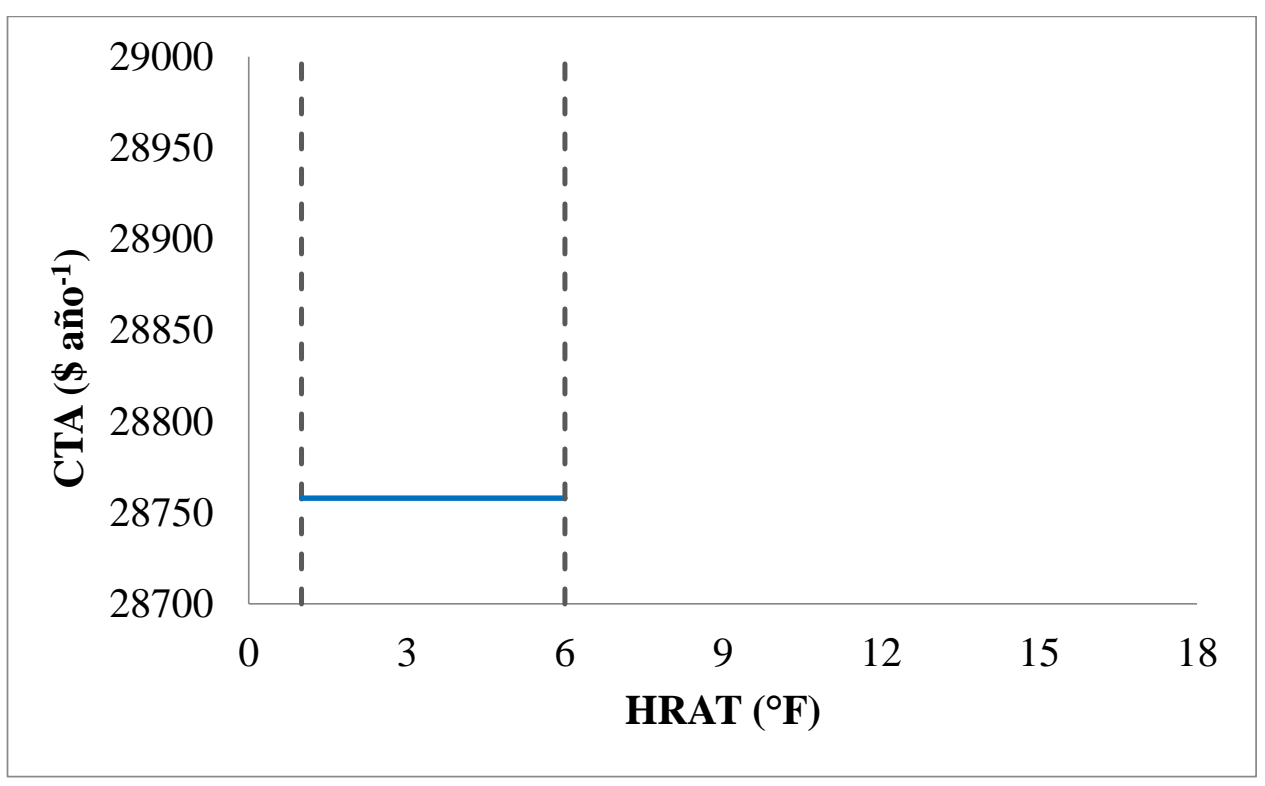

Figura 5.19. "Super-Targeting" y selección del intervalo de HRAT para la generación de topologías redundantes para el Caso de Estudio 5.4.

\section{Generación y optimización de topologías redundantes}

En la Tabla 5.20 se describen los resultados obtenidos después de optimizar las topologías redundantes del Caso de Estudio 5.4. En este problema se distingue que el primer nivel de redundancia genera un número importante de equipos, pero por otro lado la capacidad de determinar factibilidad en considerables inicializaciones se ve deteriorada en comparación con las estructuras que presentan menos equipos. En contraparte, a pesar de que mediante el tercer nivel de redundancia se incide sobre soluciones factibles en casi todas las inicializaciones, la cantidad de óptimos locales diferentes es menor en comparación con los otros dos niveles. El segundo nivel es el que presenta las mejores características determinando la mayor cantidad de óptimos locales diferentes.

En la Figura 5.20 se describen los óptimos locales de menor costo para cada topología redundante generada, permitiendo apreciar 16 óptimos locales diferentes que presentan un CTA 
menor al de la mejor solución de la cual se tiene registro. Coincidiendo con los resultados mostrados en la Tabla 5.20, el mejor diseño se obtiene en el segundo nivel de redundancia que determinó la mayor cantidad de óptimos locales diferentes, generándose mediante un HRAT de

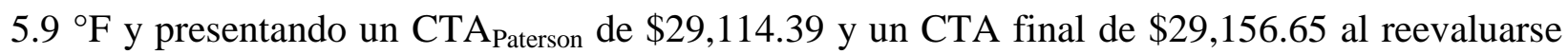
con la DMTL rigurosa. Esta solución presenta un costo $1 \%$ por debajo de la solución reportada por Luo y col. (2004). Las alternativas para disminuir el costo de las soluciones, propuestas en secciones anteriores, no son exploradas en este caso de estudio debido a que la solución determinada supera a la mejor solución reportada en la literatura.

Tabla 5.20. Resultados del Caso de Estudio 5.4.

Nivel de redundancia Primer nivel Segundo nivel Tercer nivel

\begin{tabular}{lccc}
\hline Topologías redundantes generadas & 60 & 60 & 60 \\
Número de inicializaciones por diseño base & 1000 & 1000 & 1000 \\
Soluciones factibles determinadas & 17933 & 27805 & 41299 \\
Óptimos locales diferentes & 3017 & 3632 & 1103 \\
Promedio de equipos en topologías redundantes & 58 & 40 & 18 \\
Promedio de equipos en diseños óptimos & 10 & 10 & 9 \\
Tiempo de CPU promedio por inicialización & $0.06103 \mathrm{~s}$ & $0.04213 \mathrm{~s}$ & $0.02667 \mathrm{~s}$ \\
Tiempo total de CPU & $50.86 \mathrm{~min}$ & $34.405 \mathrm{~min}$ & $20.45 \mathrm{~min}$ \\
\hline
\end{tabular}

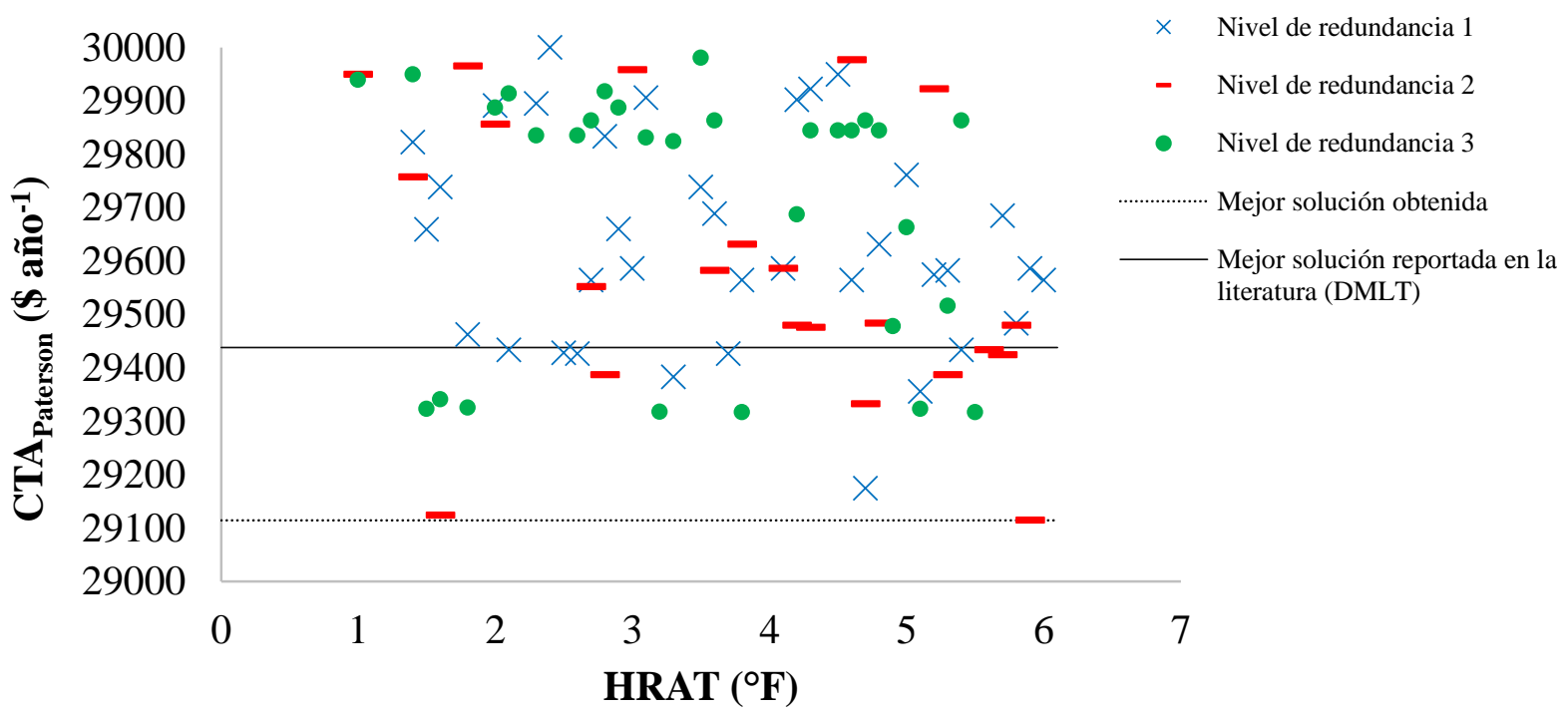

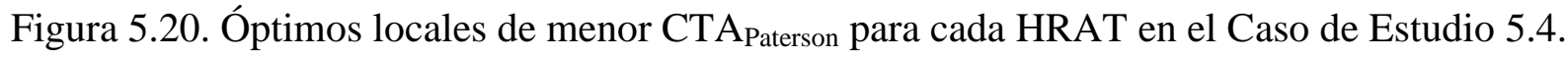


Un análisis que no se aprecia en la Figura 5.20, es que al sobrepasar el HRAT de $6{ }^{\circ} \mathrm{F}$, si es posible generar y optimizar topologías redundantes, pero los óptimos locales de dichas estructuras proporcionan costos que sobrepasan los $\$ 40,000$ por año, lo que se encuentra muy por encima del promedio de soluciones determinadas por otros autores. Esto sugiere que pueden darse casos donde resulta verdaderamente crítico realizar una correcta selección del intervalo de HRAT para determinar estructuras con posibilidades de contener soluciones competitivas.

En la Figura 5.21 se presenta la topología redundante generada con un HRAT de $5.9^{\circ} \mathrm{F}$ y el segundo nivel de redundancia la cual contiene la mejor solución óptima local determinada. La estructura presenta 42 intercambiadores de calor de los cuales 39 son recuperadores de calor y se presentan servicios de enfriamiento en las corrientes de proceso frías $\mathrm{C} 1, \mathrm{C} 2$ y C3. Se requieren 1700 variables y 1557 restricciones para su planteamiento en el modelo de PNL. La mejor solución se detalla en la Figura 5.22, presentando una estructura óptima reducida con siete recuperadores y un calentador en la corriente $\mathrm{C} 2$.

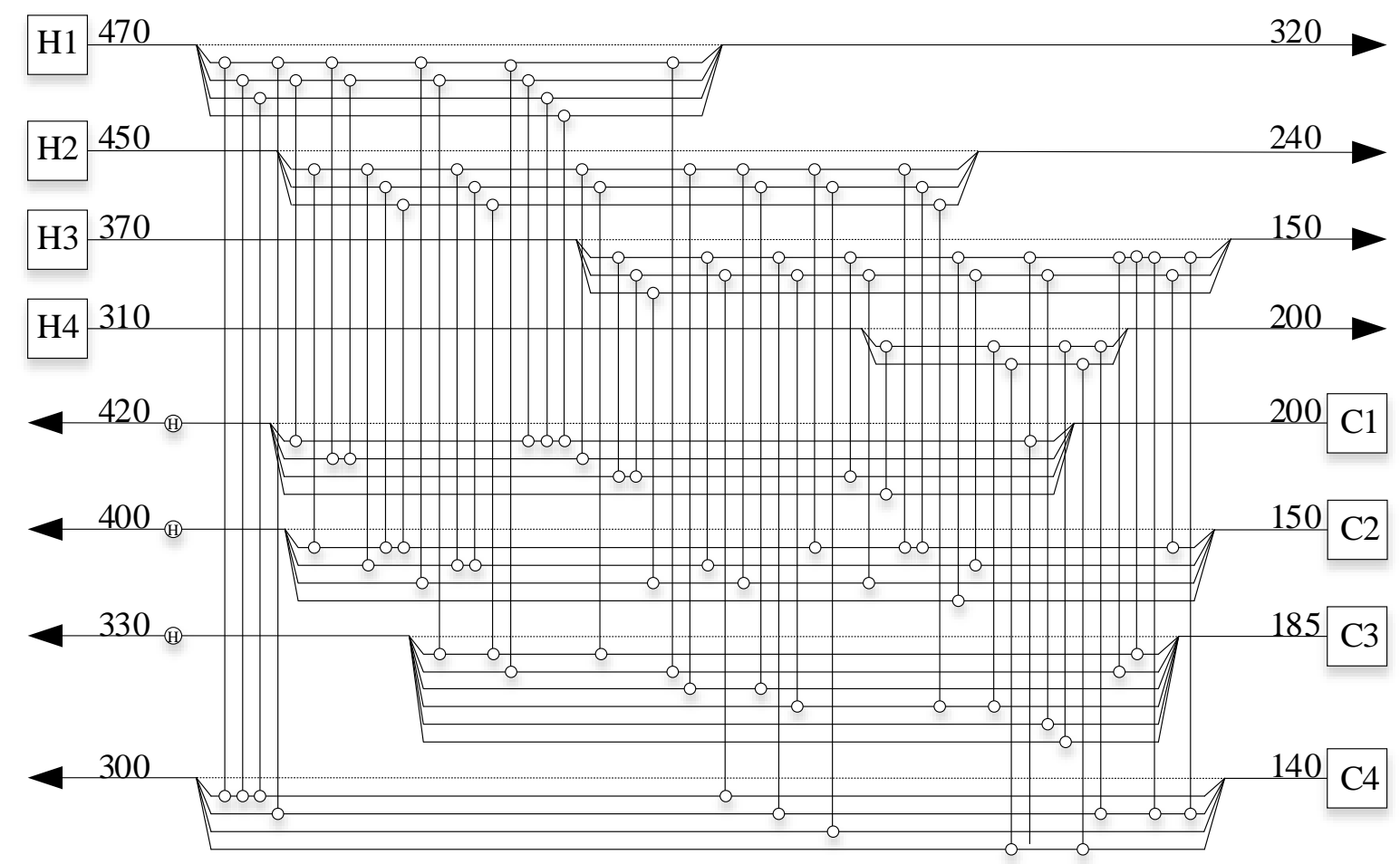

Figura 5.21. Topología redundante generada con un HRAT de $5.9^{\circ} \mathrm{F}$ y el segundo nivel de redundancia para el Caso de Estudio 5.4. 


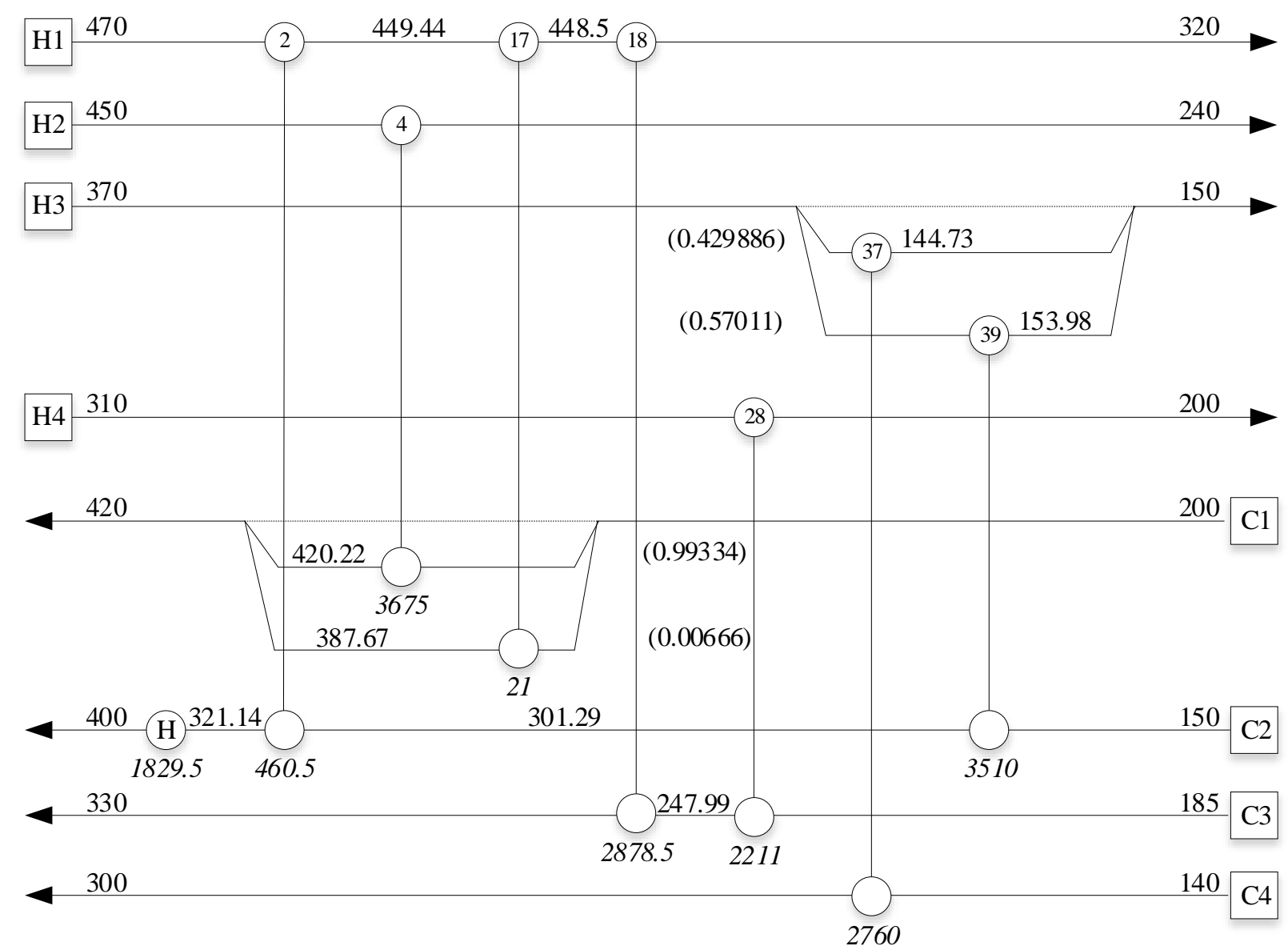

Figura 5.22. Mejor solución óptima local para el Caso de Estudio 5.4 con CTA de $\$ 29,156.65$ (temperaturas $\left({ }^{\circ} \mathrm{F}\right)$ en fuente normal, cargas térmicas $\left(\mathrm{kBtu} \mathrm{h}^{-1}\right)$ en cursivas y fracciones de flujo entre paréntesis).

\section{Comparación de resultados}

En la Tabla 5.21 se detallan los resultados obtenidos por autores que han abordado el Caso de Estudio 5.4 comparados con la mejor solución obtenida en este trabajo. 
Tabla 5.21. Comparación del Caso de Estudio 5.4 con soluciones reportadas en la literatura.

\begin{tabular}{|c|c|c|c|}
\hline Autor(es) & Metodología & Configuración ${ }^{1}$ & $\begin{array}{l}\text { CTA Reportado } \\
\qquad \begin{array}{c}\text { año } \\
-1\end{array}\end{array}$ \\
\hline $\begin{array}{l}\text { Grossman y Sargent } \\
\text { (1978) }\end{array}$ & $\begin{array}{c}\text { Algoritmo de PNL de dos } \\
\text { etapas }\end{array}$ & - & 31,890 \\
\hline $\begin{array}{l}\text { Maruki y Hayakawa } \\
\text { (1982) }\end{array}$ & $\begin{array}{c}\text { Algoritmo evolucionario de } \\
\text { tres etapas }\end{array}$ & - & 38,303 \\
\hline Luo y col. $(2004)^{2}$ & $\begin{array}{c}\text { Algoritmos } \\
\text { genéticos/Recocido } \\
\text { simulado }\end{array}$ & $6,2,0-2(4)$ & 29,438 \\
\hline He y Cui (2013) & Factor de uniformidad & $6,4,0-0(0)$ & 30,793 \\
\hline Zhang y col. (2017) & $\begin{array}{l}\text { Modelo por etapas de } \\
\text { tablero de ajedrez }\end{array}$ & $7,3,0-0(0)$ & 30,783 \\
\hline Este trabajo & Topologías redundantes & $7,1,0-2(4)$ & $29,156.65$ \\
\hline
\end{tabular}

\subsection{Caso de Estudio 5.5}

El quinto caso de estudio es el conocido problema 9SP de una planta aromática propuesto por Linnhoff y Ahmad (1990). Este problema es uno de los casos de estudio más abordados en el tema por diferentes grupos de investigación. El problema posee cuatro corrientes calientes y cinco frías. Los datos de corrientes y costos se detallan en la Tabla 5.22.

Linnhoff y Ahmad (1990) resolvieron el problema mediante la metodología de punto de pliegue consiguiendo determinar dos diseños con costo reportado de $\$ 2,930,000$ y $\$ 2,890,000$ por año. Posteriormente Zhu y col. (1995) retoman el problema y corrigen el CTA reportado de las soluciones de Linnhoff y Ahmad (1990) cuyos costos ascienden a \$2,992,000 y \$2,960,000 por año respectivamente. Estos autores utilizan un modelo de descomposición por bloques para determinar una solución con CTA de $\$ 2,980,000$. Lewin (1998) aplica por primera vez algoritmos genéticos combinado con PNL para la síntesis de redes de intercambio de calor obteniendo una solución utilizando la aproximación de Chen (Chen, 1987) para la DMLT obteniendo una solución con $\mathrm{CTA}_{\text {Chen }}$ de $\$ 2,936,000$. Petterson (2005) desarrolla un método 
secuencial de linealización por tramos para determinar la solución que se ha mantenido como la más económica hasta el momento con CTA de \$2,904,953. Bergamini y col. (2007) resuelve el problema utilizando un método de optimización global determinista con un método de relajación por partes sin permitir división de corrientes y utilizando la aproximación de Paterson para la DMLT, la solución final de estos autores tiene un CTAPaterson de \$2,935,104. Núñez-Serna y Zamora (2016) utilizaron el método de multi-arranque estocástico en dos fases para mejorar un diseño base y determinar una solución óptima con CTA de \$2,932,817 sin usar división de corrientes. Recientemente Pavão y col. (2017b) utilizan un método híbrido incorporando recocido simulado para definir las combinaciones entre corrientes y una técnica de optimización de fuegos artificiales para desarrollar una solución de \$2,919,675 por año.

Tabla 5.22. Datos de corrientes y costos para el Caso de Estudio 5.5.

\begin{tabular}{ccccc}
\hline Corriente & $\begin{array}{c}\text { Temperatura de } \\
\text { suministro } \\
\mathrm{T}\left({ }^{\circ} \mathrm{C}\right)\end{array}$ & $\begin{array}{c}\text { Temperatura } \\
\text { objetivo } \\
\mathrm{T}\left({ }^{\circ} \mathrm{C}\right)\end{array}$ & $\begin{array}{c}\text { Flujo de } \\
\text { capacidad } \\
\text { calorífica } \\
\mathrm{F}\left(\mathrm{kW}^{\circ} \mathrm{C}^{-1}\right)\end{array}$ & $\begin{array}{c}\text { Coeficiente de } \\
\text { película } \\
\mathrm{h}\left(\mathrm{kW} \mathrm{m}^{-2}{ }^{\circ} \mathrm{C}^{-1}\right)\end{array}$ \\
\hline $\mathrm{H} 1$ & 327 & 40 & 100 & 0.50 \\
$\mathrm{H} 2$ & 220 & 160 & 160 & 0.40 \\
$\mathrm{H} 3$ & 220 & 60 & 60 & 0.14 \\
$\mathrm{H} 4$ & 160 & 45 & 400 & 0.30 \\
$\mathrm{C} 1$ & 100 & 300 & 100 & 0.35 \\
$\mathrm{C} 2$ & 35 & 164 & 70 & 0.70 \\
$\mathrm{C} 3$ & 85 & 138 & 350 & 0.50 \\
$\mathrm{C} 4$ & 60 & 170 & 60 & 0.14 \\
$\mathrm{C} 5$ & 140 & 300 & 200 & 0.60 \\
$\mathrm{HU}$ & 330 & 250 & - & 0.50 \\
$\mathrm{CU}$ & 15 & 30 & - & 0.50 \\
\hline
\end{tabular}

Costo de todos los equipos $\left(\$\right.$ año $\left.^{-1}\right)=10,000+350\left[\text { Área }\left(\mathrm{m}^{2}\right)\right]^{0.6}$

Tiempo de vida de la planta $=5$ años; Tasa de interés $=0 \%$

Costo de servicios de calentamiento $=60\left(\$ \mathrm{~kW}^{-1} \mathrm{año}^{-1}\right)$

Costo de servicios de enfriamiento $=6\left(\$ \mathrm{~kW}^{-1} \mathrm{año}^{-1}\right)$

\section{Elección de parámetros de entrada}

Los resultados del método de "Super-Targeting" para el Caso de Estudio 5.5, estiman el valor del HRAT óptimo en $25^{\circ} \mathrm{C}$ como se puede ver en la Tabla 5.23. En la Figura 5.23 se muestra la 
selección del intervalo de HRAT que comprende desde un $\operatorname{HRAT}^{\mathrm{L}}=24{ }^{\circ} \mathrm{C}$ hasta un HRAT $^{\mathrm{U}}=26^{\circ} \mathrm{C}$ con un tamaño de paso $t=0.1^{\circ} \mathrm{C}$.

Tabla 5.23. Valores estimados por el método de "Super-Targeting" para el Caso de Estudio 5.5.

\begin{tabular}{ccccc}
\hline $\begin{array}{c}\text { HRAT } \\
\left({ }^{\circ} \mathrm{C}\right)\end{array}$ & $\begin{array}{c}\text { Calor } \\
\text { recuperado } \\
\text { qrec }(\mathrm{kW})\end{array}$ & $\begin{array}{c}\text { Servicios de } \\
\text { calentamiento } \\
\text { qhu }(\mathrm{kW})\end{array}$ & $\begin{array}{c}\text { Servicios de } \\
\text { enfriamiento } \\
\text { qcu }(\mathrm{kW})\end{array}$ & $\begin{array}{c}\text { CTA } \\
\text { estimado } \\
\left(\$ \text { año }^{-1}\right)\end{array}$ \\
\hline 10 & 68900 & 17280 & 25000 & $3,594,078.06$ \\
20 & 64500 & 21680 & 29400 & $3,001,087.17$ \\
24 & 62260 & 23920 & 31640 & $2,945,433.35$ \\
$\mathbf{2 5}$ & $\mathbf{6 1 7 0 0}$ & $\mathbf{2 4 4 8 0}$ & $\mathbf{3 2 2 0 0}$ & $\mathbf{2 , 9 4 2 , 8 1 7 . 3 6}$ \\
26 & 61140 & 25040 & 32760 & $2,943,589.65$ \\
30 & 58900 & 27280 & 35000 & $2,973,350.61$ \\
\hline
\end{tabular}

Se decide asignar 2000 inicializaciones por cada diseño base, debido a que es probable que el incremento en el número de corrientes involucre un mayor número de intercambiadores de calor en las estructuras de red redundantes con respecto a los casos de estudio previamente abordados en este trabajo. Por esto, resulta prudente evaluar un mayor número de puntos de arranque con el propósito de disminuir los riesgos de omitir, dentro de lo posible, soluciones óptimas locales de calidad.

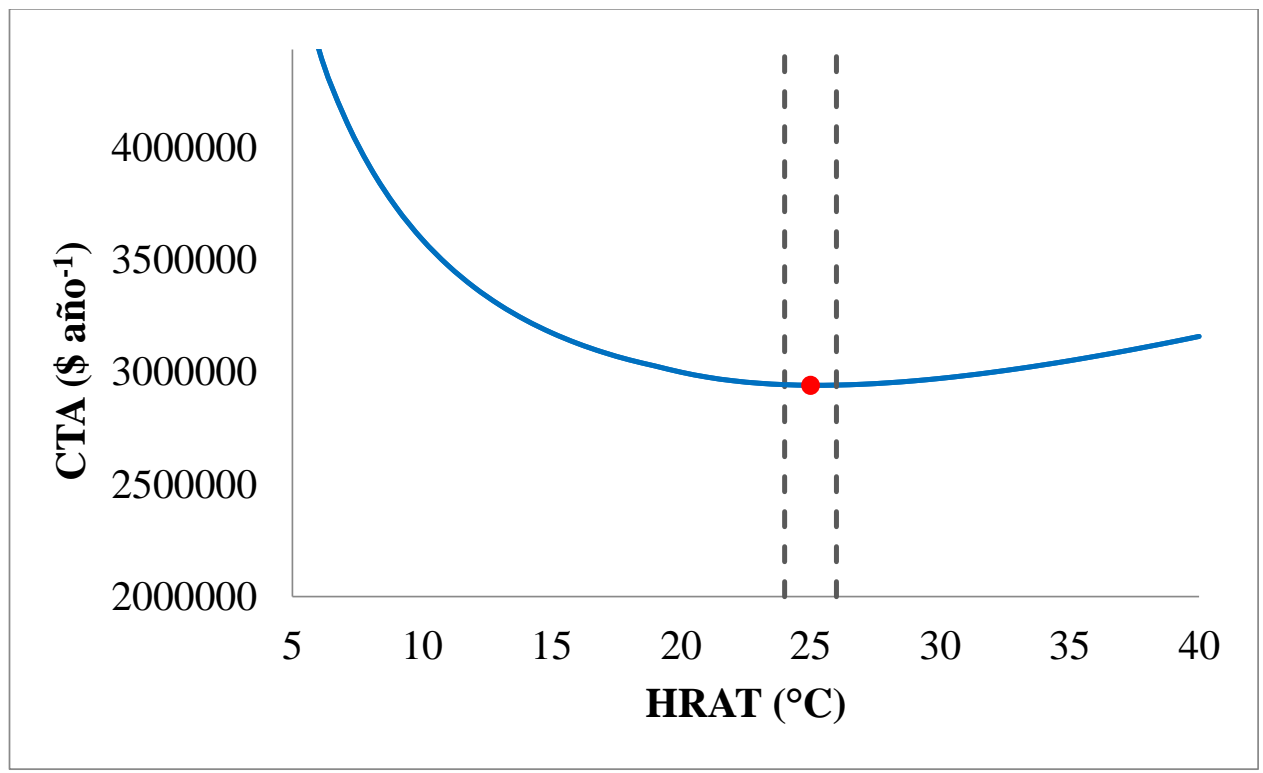

Figura 5.23. "Super-Targeting" y selección del intervalo de HRAT para la generación de topologías redundantes para el Caso de Estudio 5.5. 


\section{Generación y optimización de topologías redundantes}

Mediante el conjunto de parámetros seleccionados se generan y optimizan diferentes topologías redundantes cuyos resultados se encuentran sintetizados en la Tabla 5.24.

Tabla 5.24. Resultados del Caso de Estudio 5.5.

Nivel de redundancia

Primer nivel Segundo nivel Tercer nivel

Topologías redundantes generadas

21

Número de inicializaciones por diseño base

2000

Soluciones factibles determinadas

39126

1224

48

14

21

21

Óptimos locales diferentes

Promedio de equipos en topologías redundantes

Promedio de equipos en diseños óptimos

14

Tiempo de CPU promedio por inicialización

$0.04595 \mathrm{~s}$

2000

2000

Tiempo total de CPU

$32.163 \mathrm{~min} \quad 21.898 \mathrm{~min}$

1050

41881

936

$35 \quad 24$

$15 \quad 13$

Las soluciones con menor costo para cada HRAT se detallan en la Figura 5.24, siendo la mejor aquella que presenta un $\mathrm{CTA}_{\text {Paterson }}$ asociado de $\$ 2,930,288.49$. La red de intercambio de calor de menor costo es generada en el segundo nivel de redundancia utilizando un HRAT de $24.9^{\circ} \mathrm{F}$; el diseño de red presenta 19 equipos, de entre los cuales 14 son recuperadores de calor, requiriendo servicios de calentamiento en las corrientes $\mathrm{C} 1$ y $\mathrm{C} 5$, y servicios de calentamiento en las corrientes $\mathrm{H} 1, \mathrm{H} 3$ y H4.

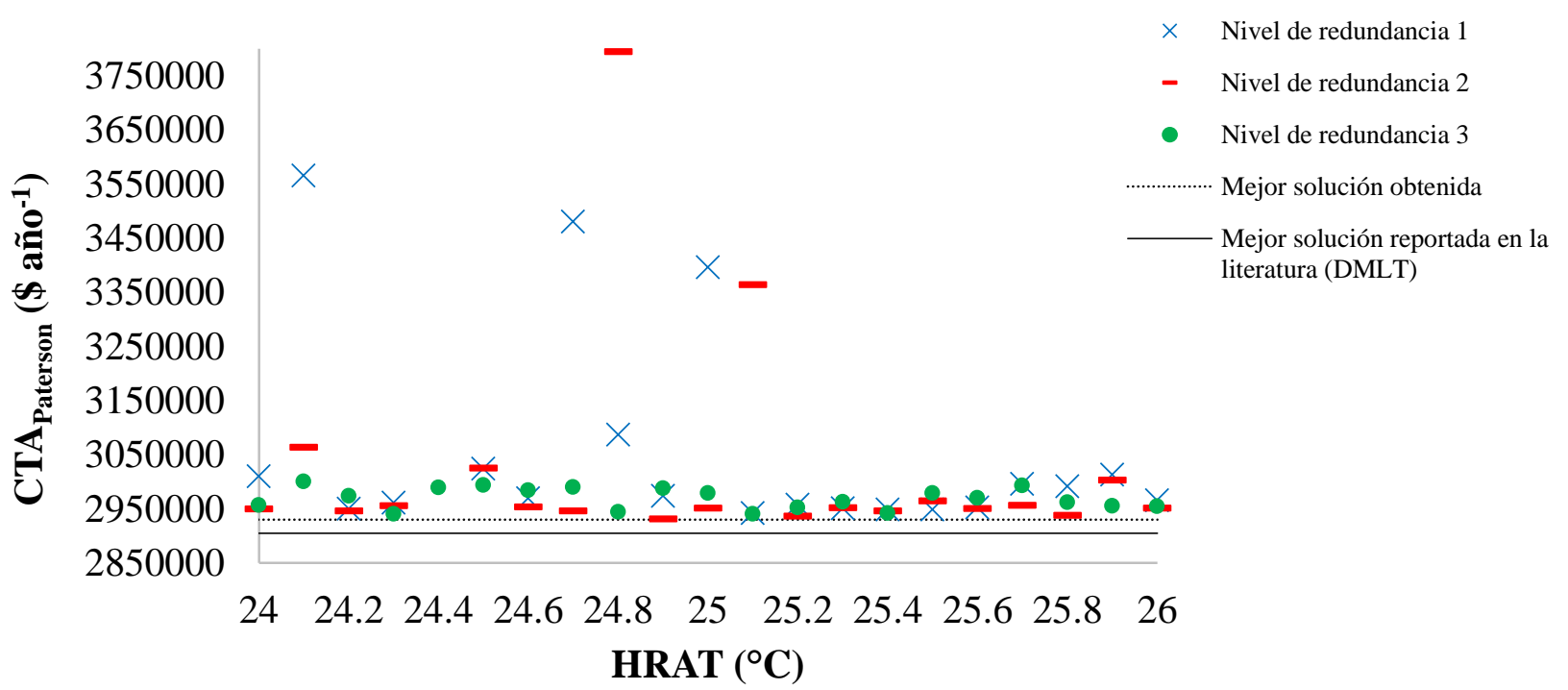

Figura 5.24. Óptimos locales de menor CTAPaterson para cada HRAT en el Caso de Estudio 5.5. 


\section{Generación y optimización de topologías redundantes restringiendo servicios auxiliares}

Se procede a generar topologías redundantes prohibiendo los servicios auxiliares no requeridos por la mejor solución previamente determinada. Se imponen costos de transporte altos asociados a estas corrientes de forma que $C C U_{2, l}=M, C H U_{2, l 1}=M, C H U_{3, l 1}=M$ y $C H U_{4, l 1}=M$. Las soluciones determinadas en esta prueba tienen un CTA muy elevado, casi en su mayoría presentando costos superiores a los diseños sin división de corrientes desarrollados por otros autores. En busca de compensar este inconveniente se propone también evaluar un mayor número de diseños iniciales cambiando el incremento en el HRAT a $t=0.01{ }^{\circ} \mathrm{C}$, y aumentando el número de inicializaciones a 5000. Por otro lado, debido a que el tiempo de cómputo comienza a verse afectado de forma negativa por el tamaño del problema, la prueba de diversificación e intensificación se diseña únicamente para el primer nivel de redundancia. En la Tabla 5.18 se muestran los datos resultantes para la prueba con servicios auxiliares limitados.

Tabla 5.25. Resultados del Caso de Estudio 5.5 prohibiendo servicios auxiliares a las corrientes de proceso e intensificando la búsqueda.

\begin{tabular}{lc}
\cline { 2 - 2 } & Primer nivel \\
\hline Topologías redundantes generadas & 201 \\
Número de inicializaciones por diseño base & 5000 \\
Soluciones factibles determinadas & 969135 \\
Óptimos locales diferentes & 15711 \\
Promedio de equipos en topologías redundantes & 49 \\
Promedio de equipos en diseños óptimos & 13 \\
Tiempo de CPU promedio por inicialización & $0.05071 \mathrm{~s}$ \\
Tiempo total de CPU & $14.155 \mathrm{hrs}$ \\
\hline
\end{tabular}

Los costos de los óptimos locales representativos de cada HRAT se muestran en la Figura 5.25. Para este problema a pesar de realizar una considerable inversión de recursos computacionales no fue posible obtener un diseño con CTA equiparable con la mejor solución reportada en la

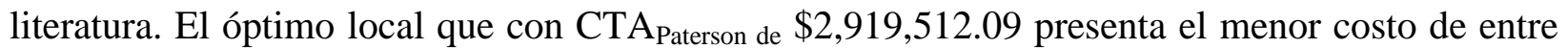
todas las soluciones obtenidas. La reevaluación con la DMLT proporciona un CTA final de $\$ 2,919,688.66$ la cual se encuentra $0.5 \%$ por encima de la mejor solución reportada en la literatura desarrollada por Petterson (2005). Una ventaja que presenta la solución obtenida en 
este trabajo es que requiere una menor cantidad de intercambiadores de calor con un total de 12 unidades, en comparación con la solución determinada por Petterson (2005) la cual alcanza las 17 unidades. Otra ventaja que exhibe la solución determinada en este trabajo, es que presenta una estructura considerablemente menos compleja al requerir solamente tres divisores de corriente con seis sub-corrientes en total, mientras que la solución de Petterson (2005) requiere de siete divisores y un total de catorce sub-corrientes de proceso. Otra característica de la solución obtenida por estos autores es que utilizan una misma corriente de calentamiento externo para calentar la corriente de proceso C5 en diferentes secciones. Esta propiedad no se puede reproducir en la mayoría de las metodologías y formulaciones de síntesis al no disponer de una cantidad que represente el flujo de capacidad calorífica de los servicios auxiliares que permita calcular la temperatura a la salida del primer intercambiador que interactúa con el servicio auxiliar.

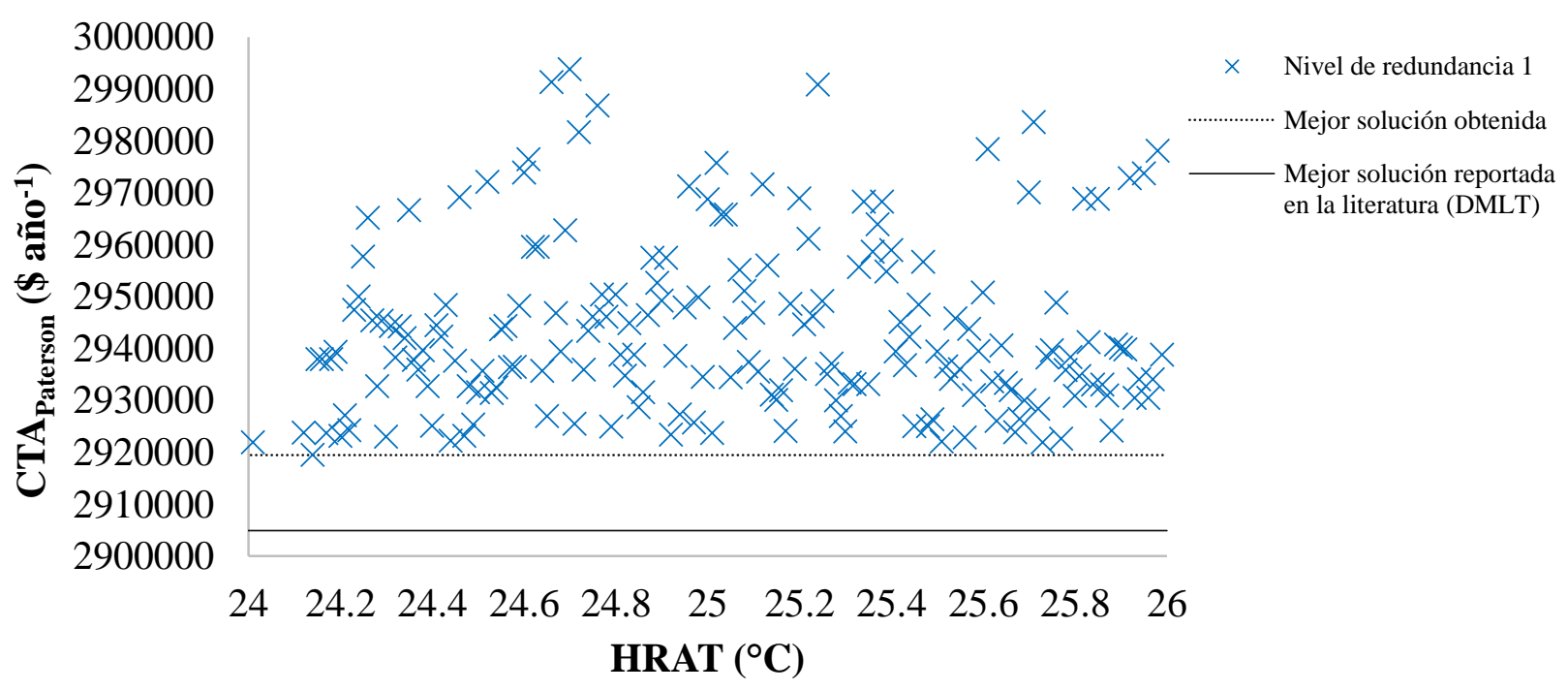

Figura 5.25. Búsqueda intensiva de soluciones óptimas locales para cada HRAT en el Caso de Estudio 5.5, prohibiendo servicios auxiliares a las corrientes de proceso $\mathrm{H} 2, \mathrm{C} 2, \mathrm{C} 3$ y C4.

Por otro lado, el diseño obtenido en este trabajo presenta un costo similar a la solución determinada por Pavão y col. (2017) quienes recientemente determinaron una solución con CTA reportado de \$2,919,675 colocándose como los autores con la segunda mejor solución disponible en la literatura, siendo únicamente $\$ 13$ por año más barata que el diseño obtenido en este trabajo. El hecho de que no se hayan conseguido soluciones cercanas a la determinada por Petterson 
(2008), aun siendo un caso de estudio recurrentemente abordado, sugiere que es un problema particularmente difícil de resolver.

La topología con HRAT de $24.14{ }^{\circ} \mathrm{C}$ que contiene a la mejor solución obtenida en este trabajo se muestra en la Figura 5.26. La formulación de esta estructura requiere 1814 variables y 1666 restricciones para el modelo de PNL asociado. El diseño final simplificado más económico obtenido en este trabajo con CTA de \$2,919,687.66 se ilustra en la Figura 5.27.

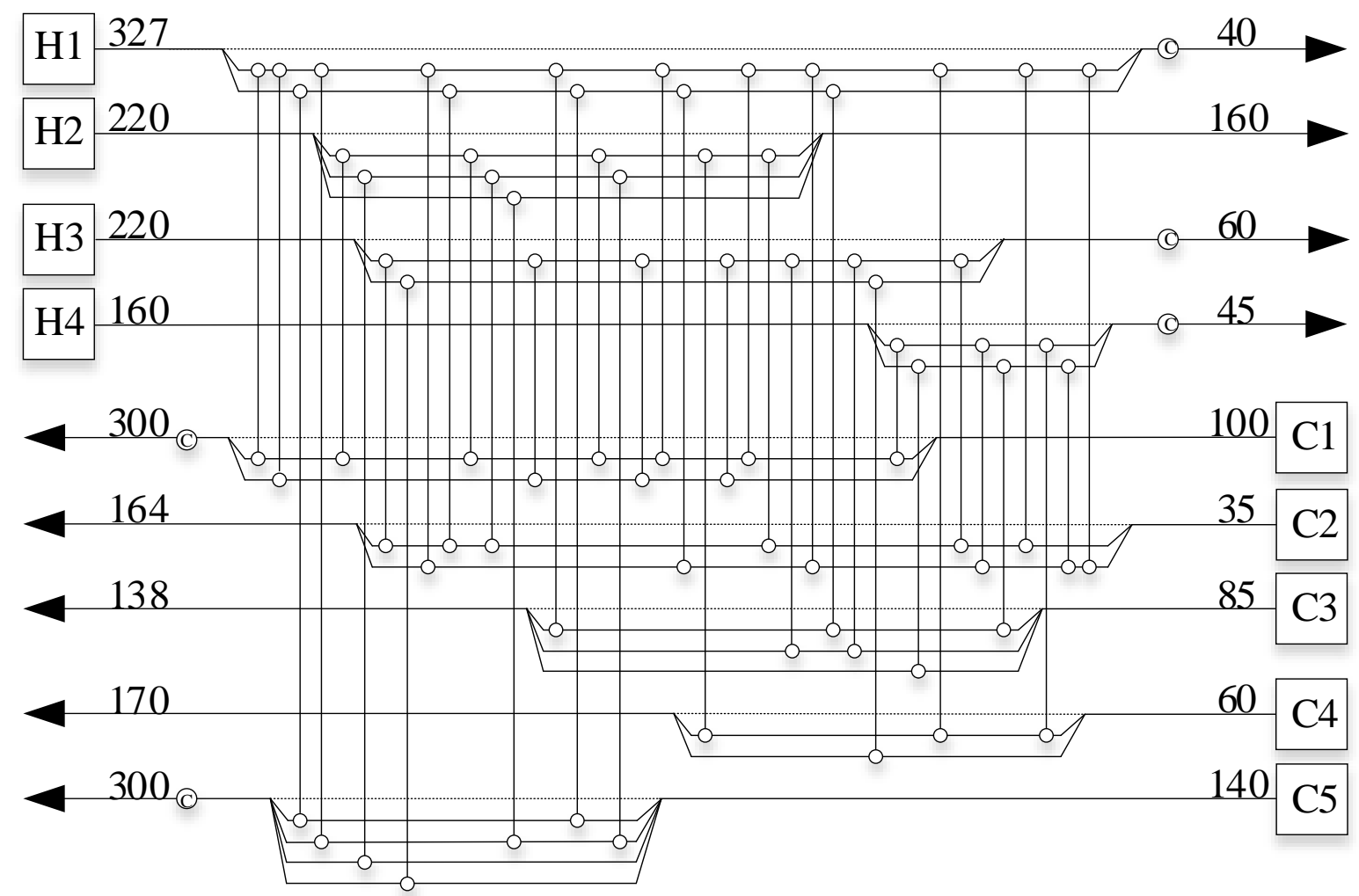

Figura 5.26. Topología redundante generada con un HRAT de $12{ }^{\circ} \mathrm{C}$ y una búsqueda intensiva usando el primer nivel de redundancia para el Caso de Estudio 5.5. 


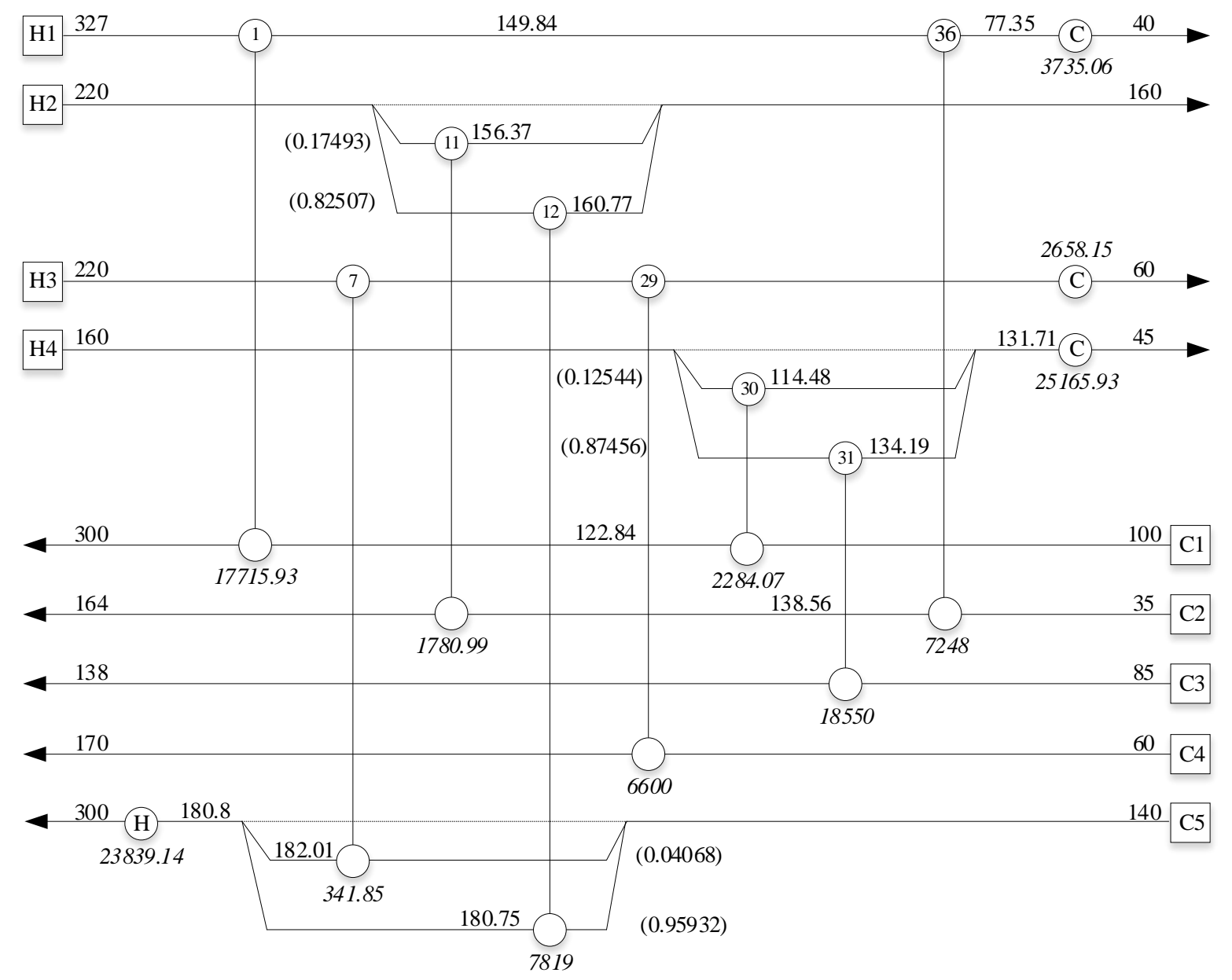

Figura 5.27. Mejor solución óptima local para el Caso de Estudio 5.5 con CTA de \$2,919,687.66 (temperaturas $\left({ }^{\circ} \mathrm{C}\right)$ en fuente normal, cargas térmicas $(\mathrm{kW})$ en cursivas y fracciones de flujo entre paréntesis). 


\section{Comparación de resultados}

En la Tabla 5.26 se detallan los resultados obtenidos por autores que han abordado el Caso de Estudio 5.5 comparados con la mejor solución obtenida en este trabajo.

Tabla 5.26. Comparación del Caso de Estudio 5.5 con soluciones reportadas en la literatura.

\begin{tabular}{|c|c|c|c|}
\hline Autor(es) & Metodología & Configuración ${ }^{1}$ & $\begin{array}{l}\text { CTA Reportado } \\
\qquad \text { año }^{-1}\end{array}$ \\
\hline Linnhoff y Ahmad (1990) & Punto de Pliegue & $7,3,3-0(0)$ & $2,960,522$ \\
\hline Zhu y col. (1995) & Descomposición por bloques & $5,2,3-0(0)$ & $2,984,000$ \\
\hline Lewin (1998) & Algoritmos genéticos & $7,2,3-2(4)$ & $2,936,000^{* * *}$ \\
\hline \multirow{3}{*}{ Petterson $(2005)^{2}$} & \multirow{3}{*}{ Linealización por tramos } & $11,3,3-7(14)$ & $2,904,953$ \\
\hline & & $10,3,3-6(12)$ & $2,919,044$ \\
\hline & & $8,3,3-3(6)$ & $2,930,390$ \\
\hline Bergamini (2007) & $\begin{array}{c}\text { Superestructura-Optimización } \\
\text { Global }\end{array}$ & $10,2,3-0(0)$ & $2,935,104^{*}$ \\
\hline $\begin{array}{l}\text { Yerramsetty y Murty } \\
(2008)\end{array}$ & Evolución diferencial & $10,2,3-0(0)$ & $2,942,000 * *$ \\
\hline Luo y col. (2009) & Algoritmos genéticos & $9,2,3-3(6)$ & $2,924,298$ \\
\hline Toffolo (2009) & Topologías sin restricciones & $11,1,3-4(8)$ & $2,920,000$ \\
\hline Huo y col. (2013) & Algoritmos genéticos & $9,1,3-2(4)$ & $2,922,600 * *$ \\
\hline Peng y Cui (2015) & Recocido simulado & $10,2,3-0(0)$ & $2,935,000$ \\
\hline $\begin{array}{l}\text { Núñez-Serna y Zamora } \\
(2016)\end{array}$ & Optimización multi-arranque & $10,2,3-0(0)$ & $2,932,817$ \\
\hline Chen y col. (2017) & Evolución diferencial & $10,2,3-0(0)$ & $2,924,592$ \\
\hline \multirow{3}{*}{ Pavão y col. $(2017 b)^{2}$} & \multirow{3}{*}{$\begin{array}{l}\text { Recocido simulado-Fuegos } \\
\text { artificiales }\end{array}$} & $8,1,3-2(4)$ & $2,919,675$ \\
\hline & & $7,1,3-2(4)$ & $2,920,073$ \\
\hline & & $10,1,3-0(0)$ & $2,920,763$ \\
\hline Xiao y col. (2018) & $\begin{array}{l}\text { Algoritmo de búsqueda aleatoria } \\
\text { con evolución compulsiva }\end{array}$ & $10,2,3-0(0)$ & $2,926,766$ \\
\hline Este trabajo & Topologías redundantes & $8,1,3-2(4)$ & 2,919,687.66 \\
\hline \multicolumn{4}{|c|}{$\begin{array}{l}{ }^{1} \text { Recuperadores, Calentadores,Enfriadores-No. de Divisores de corrientes(No. de Sub-corrientes). } \\
{ }^{2} \text { Solución revisada en el Apéndice A } \\
\text { * CTA reportado con la aproximación de Paterson } \\
\text { **CTA reportado con la aproximación de Chen }\end{array}$} \\
\hline
\end{tabular}




\subsection{Caso de Estudio 5.6}

El caso de estudio 5.6 es el conocido problema 10SP1, propuesto por Pho y Lapidus (1973), el cual se constituye de 5 corrientes calientes y 5 corrientes frías. Los datos originales del problema fueron presentados en el sistema inglés de unidades y posteriormente se replantearon en el sistema internacional de unidades, siendo manejados de esta manera por la mayoría de los autores. Los datos para este problema se presentan en la Tabla 5.27. Este caso de estudio es uno de los más abordados en la literatura y ha sido utilizado como punto de referencia para probar las diferentes metodologías y técnicas de síntesis.

Tabla 5.27. Datos de corrientes y costos para el Caso de Estudio 5.6.

\begin{tabular}{ccccc}
\hline Corriente & $\begin{array}{c}\text { Temperatura de } \\
\text { suministro } \\
\mathrm{T}(\mathrm{K})\end{array}$ & $\begin{array}{c}\text { Temperatura } \\
\text { objetivo } \\
\mathrm{T}(\mathrm{K})\end{array}$ & $\begin{array}{c}\text { Flujo de } \\
\text { capacidad } \\
\text { calorífica } \\
\mathrm{F}\left(\mathrm{kW} \mathrm{K}^{-1}\right)\end{array}$ & $\begin{array}{c}\text { Coeficiente de } \\
\text { película } \\
\mathrm{h}\left(\mathrm{kW} \mathrm{m}^{-2} \mathrm{~K}^{-1}\right)\end{array}$ \\
\hline $\mathrm{H} 1$ & 433 & 366 & 8.79 & 1.704 \\
$\mathrm{H} 2$ & 522 & 411 & 10.55 & 1.704 \\
$\mathrm{H} 3$ & 544 & 422 & 12.56 & 1.704 \\
$\mathrm{H} 4$ & 500 & 339 & 14.77 & 1.704 \\
$\mathrm{H} 5$ & 472 & 339 & 17.73 & 1.704 \\
$\mathrm{C} 1$ & 355 & 450 & 17.28 & 1.704 \\
$\mathrm{C} 2$ & 366 & 478 & 13.90 & 1.704 \\
$\mathrm{C} 3$ & 311 & 494 & 8.44 & 1.704 \\
$\mathrm{C} 4$ & 333 & 433 & 7.62 & 1.704 \\
$\mathrm{C} 5$ & 389 & 495 & 6.08 & 1.704 \\
$\mathrm{HU}$ & 509 & 509 & - & 3.408 \\
$\mathrm{CU}$ & 311 & 355 & - & 1.704 \\
\hline
\end{tabular}

Costo de todos los equipos $\left(\$\right.$ año $\left.^{-1}\right)=145.63\left[\text { Área }\left(\mathrm{m}^{2}\right)\right]^{0.6}$

Costo de servicios de calentamiento $=37.64\left(\$ \mathrm{~kW}^{-1} \mathrm{año}^{-1}\right)$

Costo de servicios de enfriamiento $=18.12\left(\$ \mathrm{~kW}^{-1} \mathrm{año}^{-1}\right)$

Lin y Miller (2004) con un enfoque de búsqueda tabú desarrollaron un diseño con CTA de $\$ 43,329$. Pariyani y col. (2006) resolvieron el problema mediante algoritmos aleatorios desarrollando una solución con costo de $\$ 43,439$ por año. Petterson (2008) mediante un enfoque de optimización global basado en la superestructura Synheat, utiliza la diferencia media 
geométrica de temperaturas para sustituir la DMLT, y obtener una solución óptima de \$43,331 por año. Recientemente Wu y col. (2015) implementaron un modelo simultáneo adoptando la superestructura de Yee y Grossmann (1990) que permite mezclado no isotérmico y la capacidad de admitir flujos de capacidad caloríficas dependientes de la temperatura. Estos autores utilizaron la aproximación de Chen (Chen, 1987) para sustituir la DMLT y presentan una solución con $\mathrm{CTA}_{\mathrm{Chen}}$ reportado de $\$ 42,961$, sin embargo, la diferencia considerable de costos en comparación con otras soluciones encontradas en la literatura se debe a un error en los coeficientes de película de los servicios auxiliares. Al recalcular y reevaluar con la DMLT, el diseño de red presenta un costo revisado final de $\$ 43,319$ por año la cual aún representa la solución más económica de la que se tiene conocimiento.

\section{Elección de parámetros de entrada}

El Caso de Estudio 5.6 al igual que el abordado en la Sección 5.5 es considerado un problema de umbral, con la diferencia de que en esta ocasión solamente son requeridos servicios de enfriamiento. En la Tabla 5.28 se detallan los valores obtenidos por el método de "SuperTargeting”, y en la Figura 5.28 es posible distinguir que las metas de energía se mantienen constantes en los valores comprendidos desde el HRAT de $1 \mathrm{~K}$ hasta $39 \mathrm{~K}$. Esta característica otorga flexibilidad al momento de elegir el parámetro de diseño HRAT, pues es posible considerar a cualquier valor definido dentro de la región de umbral como óptimo. Se elige el intervalo definido desde un $\mathrm{HRAT}^{\mathrm{L}}=10 \mathrm{~K}$ hasta un $\mathrm{HRAT}^{\mathrm{U}}=15 \mathrm{~K}$ para la construcción de topologías redundantes, considerando un incremento al HRAT en cada iteración de $t=0.1 \mathrm{~K}$. Asignando 2000 inicializaciones a cada diseño base.

Tabla 5.28. Valores estimados por el método de "Super-Targeting" para el Caso de Estudio 5.6.

\begin{tabular}{ccccc}
\hline $\begin{array}{c}\text { HRAT } \\
(\mathrm{K})\end{array}$ & $\begin{array}{c}\text { Calor } \\
\text { recuperado } \\
\mathrm{q}_{\mathrm{rec}}(\mathrm{kW})\end{array}$ & $\begin{array}{c}\text { Servicios de } \\
\text { calentamiento } \\
\mathrm{qhu}(\mathrm{kW})\end{array}$ & $\begin{array}{c}\text { Servicios de } \\
\text { enfriamiento } \\
\mathrm{qcu}(\mathrm{kW})\end{array}$ & $\begin{array}{c}\text { CTA } \\
\text { estimado } \\
\left(\$ \text { año }^{-1}\right)\end{array}$ \\
\hline $\mathbf{1}$ & $\mathbf{6 1 4 9 . 4}$ & $\mathbf{0}$ & $\mathbf{1 8 7 8 . 9 6}$ & $\mathbf{4 3 6 4 1 . 2 1}$ \\
$\mathbf{3 9}$ & $\mathbf{6 1 4 9 . 4}$ & $\mathbf{0}$ & $\mathbf{1 8 7 8 . 9 6}$ & $\mathbf{4 3 6 4 1 . 2 1}$ \\
40 & 6130.4 & 19 & 1897.96 & 44647.83 \\
\hline
\end{tabular}




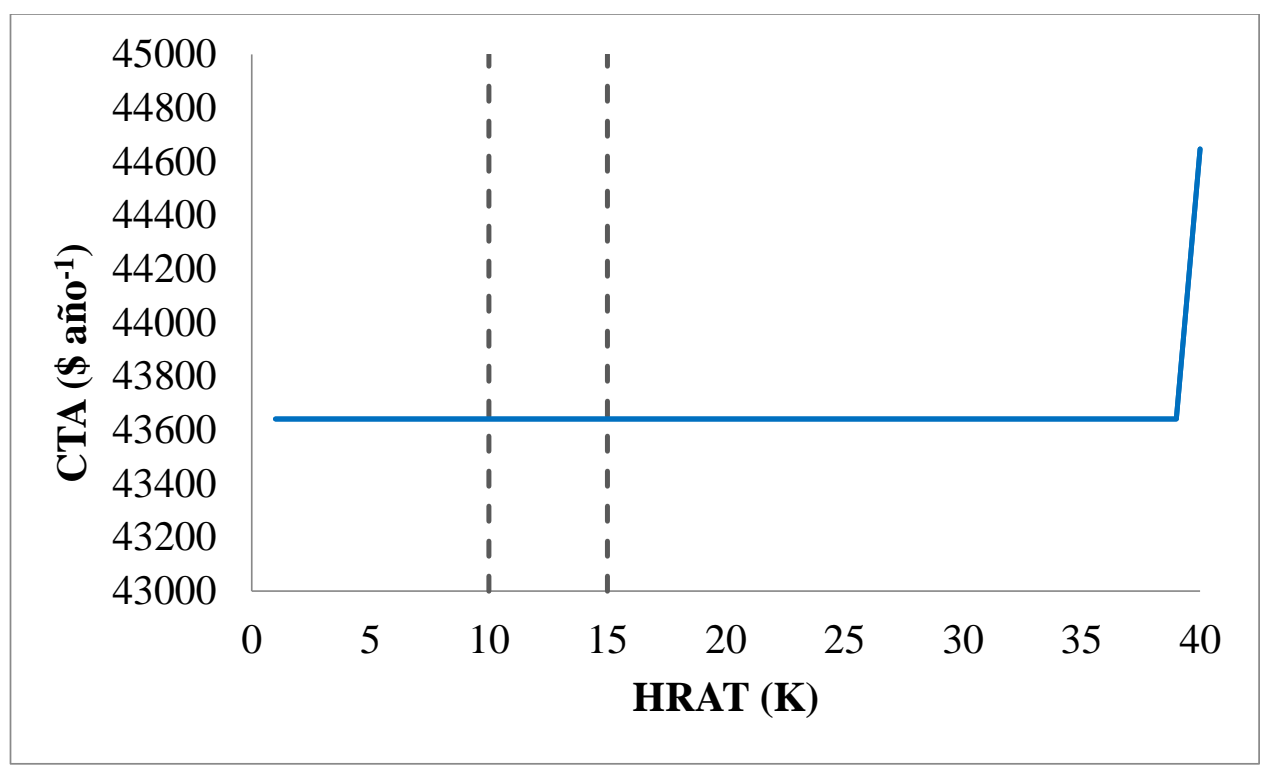

Figura 5.28. “Super-Targeting” y selección del intervalo de HRAT para la generación de topologías redundantes para el Caso de Estudio 5.6.

\section{Generación y optimización de topologías redundantes}

El resultado de optimización para las topologías redundantes generadas se detalla en la Tabla 5.21. A pesar de que este caso de estudio presenta tan solo una corriente de proceso más en comparación con el Caso de Estudio 5.6, el número de equipos disponibles en los tres niveles de redundancia se ve incrementado considerablemente. Esto se traduce en un requerimiento mayor en el uso recursos computacionales por cada iteración, lo cual se ve reflejado en el tiempo total de CPU requerido para optimizar el conjunto de diseños base.

En la Figura 5.29 se muestran las soluciones de menor costo obtenidas. El problema 10SP1 presenta la particularidad de que las soluciones evaluadas con la aproximación de Paterson (Paterson, 1984) para la DMLT exhiben un costo prácticamente equivalente a los calculados con la DMLT rigurosa. La mejor solución obtenida en este trabajo se determinó mediante la estructura de red generada con un HRAT de $10.9{ }^{\circ} \mathrm{C}$ y el primer nivel de redundancia, dicha solución presenta un $\mathrm{CTA}_{\text {Paterson }}$ de $\$ 43,323.5$ y un costo reevaluado con la DMLT de $\$ 43,323.8$ por año. Aunque la solución determinada exhibe un CTA únicamente $0.01 \%$ por encima de la mejor solución reportada en la literatura por Wu y col. (2015) con CTA revisado de \$43,319, la importancia de este caso de estudio como punto de referencia de metodologías de síntesis, 
demanda llevar al límite las capacidades del enfoque propuesto por lo que se diseña una segunda prueba en búsqueda de alguna solución de menor costo.

Tabla 5.29. Resultados del Caso de Estudio 5.6.

Nivel de redundancia Primer nivel Segundo nivel Tercer nivel

\begin{tabular}{lccc} 
Topologías redundantes generadas & 51 & 51 & 51 \\
Número de inicializaciones por diseño base & 2000 & 2000 & 2000 \\
Soluciones factibles determinadas & 81073 & 87766 & 100535 \\
Óptimos locales diferentes & 8773 & 8919 & 4870 \\
Promedio de equipos en topologías redundantes & 84 & 64 & 27 \\
Promedio de equipos en diseños óptimos & 11 & 12 & 11 \\
Tiempo de CPU promedio por inicialización & $0.15809 \mathrm{~s}$ & $0.08415 \mathrm{~s}$ & $0.04446 \mathrm{~s}$ \\
Tiempo total de CPU & $4.479 \mathrm{hrs}$ & $2.384 \mathrm{hrs}$ & $1.26 \mathrm{hrs}$ \\
\hline
\end{tabular}

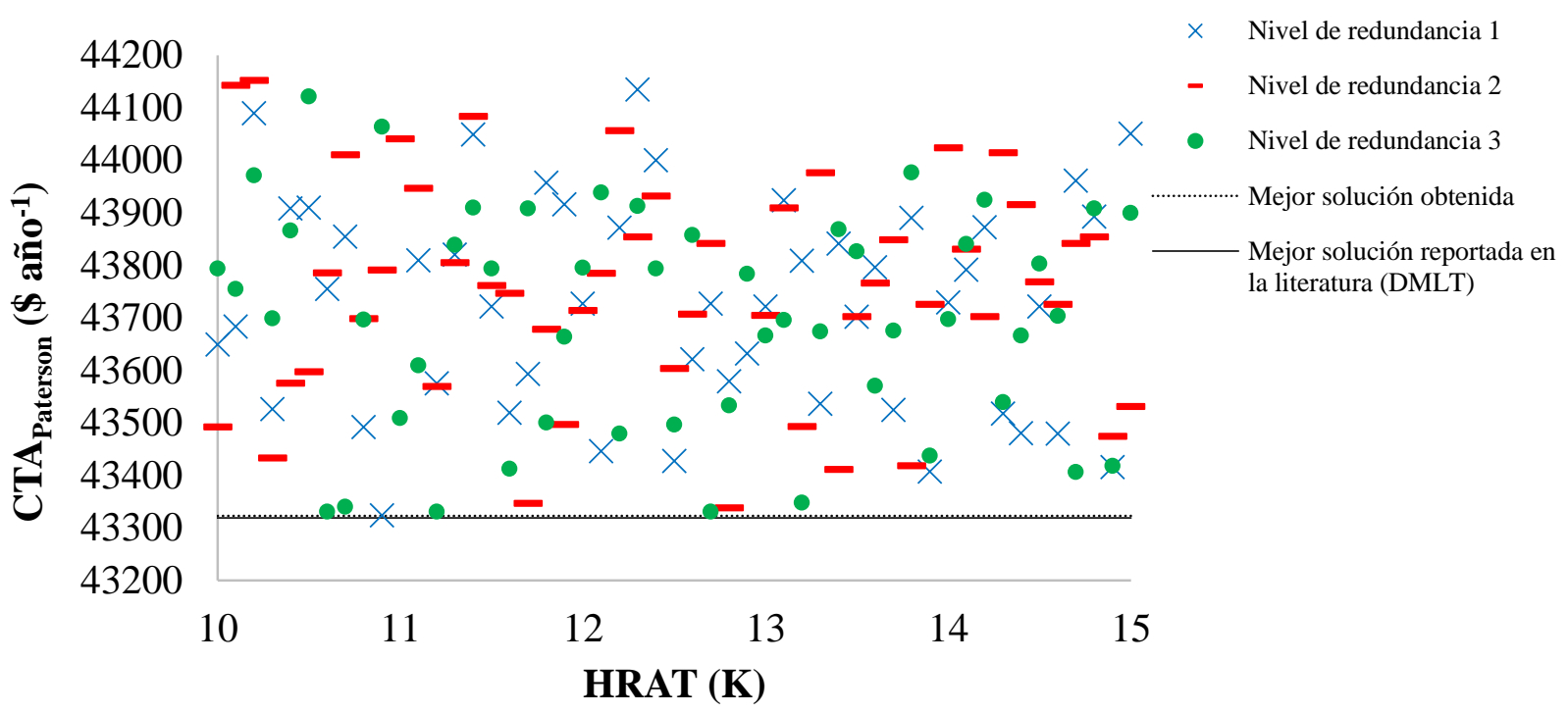

Figura 5.29. Óptimos locales de menor CTA Paterson para cada HRAT en el Caso de Estudio 5.6.

\section{Generación y optimización de topologías redundantes restringiendo servicios auxiliares}

Debido a que en este caso de estudio los recursos computacionales se encuentran comprometidos por el alto número de equipos presentes en las topologías redundantes a optimizar, se propone únicamente intensificar la búsqueda de soluciones óptimas locales sin generar una mayor cantidad de diseños iniciales, con la idea de que las topologías generadas, al tener un número 
elevado de equipos, pudieran contener óptimos locales de mejor calidad que no han sido explorados. Por otro lado, aunque el Caso de Estudio 5.6 es un problema de umbral que no requiere servicios de calentamiento, se pudo observar que algunas distribuciones de cargas térmicas otorgadas por la solución del modelo de transporte, requieren de enfriamiento en todas las corrientes calientes a pesar de que las metas de energía del "Super-Targeting" indican que solo es necesario en las corrientes de proceso $\mathrm{H} 4$ y H5, esto se confirma al identificar que todas las soluciones obtenidas eliminan los enfriadores innecesarios. Aunque se tenga cierta certidumbre de que estos equipos serán eliminados por el esquema de optimización, suponen restricciones y variables innecesarias que pueden ser evitadas desde la formulación del modelo de transporte. De esta manera se propone que $C C U_{1, l}=M, C C U_{2, l}=M$ y $C C U_{3, l}=M$ para prohibir los servicios de enfriamiento en las corrientes de proceso H1, H2 y H3 y se incrementa el número de inicializaciones a 5000 por cada diseño base a optimizar. Se utiliza únicamente el primer nivel de redundancia para la búsqueda de soluciones debido al tiempo de cómputo requerido esta prueba incrementa considerablemente. En la Tabla 5.30 se describen los resultados de la optimización.

Tabla 5.30. Resultados del Caso de Estudio 5.6 prohibiendo servicios auxiliares a las corrientes de proceso e intensificando la búsqueda.

\begin{tabular}{lc}
\cline { 2 - 2 } & Primer nivel \\
\hline Topologías redundantes generadas & 51 \\
Número de inicializaciones por diseño base & 5000 \\
Soluciones factibles determinadas & 197787 \\
Óptimos locales diferentes & 11262 \\
Promedio de equipos en topologías redundantes & 85 \\
Promedio de equipos en diseños óptimos & 11 \\
Tiempo de CPU promedio por inicialización & $0.14137 \mathrm{~s}$ \\
Tiempo total de CPU & $10.21 \mathrm{hrs}$ \\
\hline
\end{tabular}

En la Figura 5.30 se muestran los resultados en términos de costos de la optimización de las topologías redundantes generadas. La inversión de recursos computacionales se ve recompensada con una solución con un CTA ligeramente menor a la mejor solución encontrada en la literatura. La estructura redundante que la contiene, mostrada en la Figura 5.31 se genera 
con un HRAT de $12 \mathrm{~K}$ y presenta un total de 83 intercambiadores de calor, para la cual fueron necesarias 4743 variables y 4463 restricciones en la formulación del modelo de PNL.

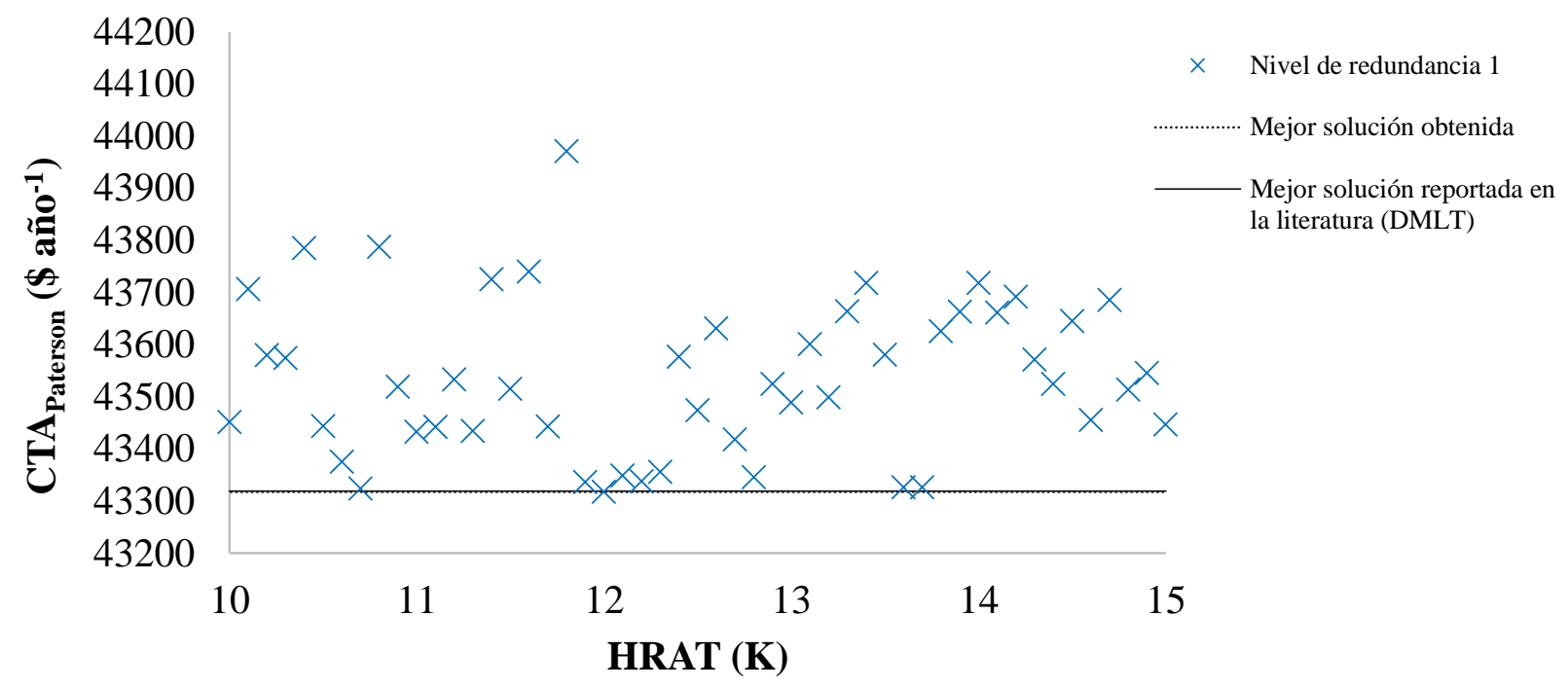

Figura 5.30. Búsqueda intensiva de soluciones óptimas locales para cada HRAT en el Caso de Estudio 5.6, prohibiendo los servicios auxiliares a las corrientes de proceso $\mathrm{H} 1, \mathrm{H} 2$ y $\mathrm{H} 3$.

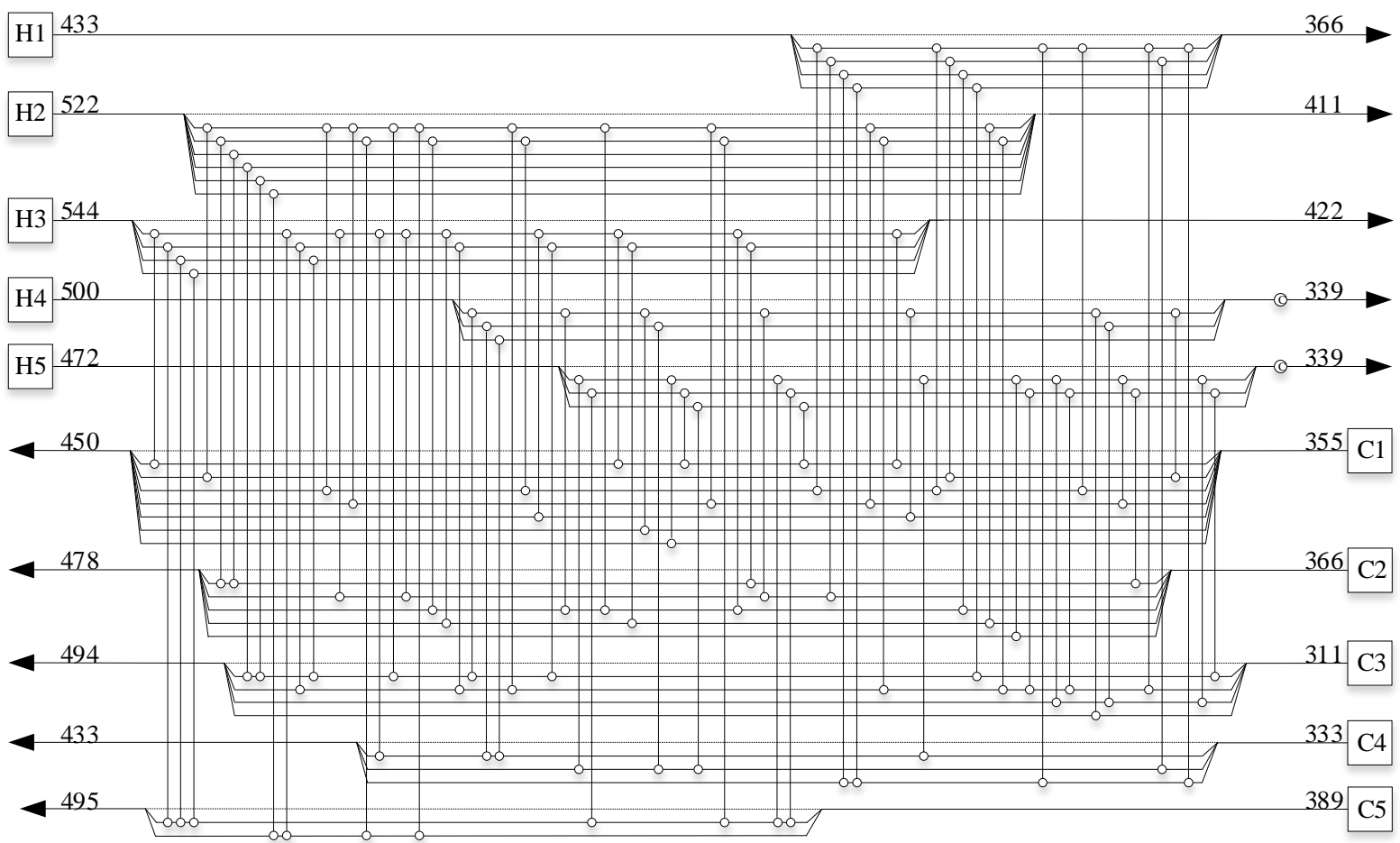

Figura 5.31. Topología redundante generada con un HRAT de $12 \mathrm{~K}$ y una búsqueda intensiva usando el primer nivel de redundancia para el Caso de Estudio 5.6. 
El óptimo local de menor costo mostrado como diseño reducido en la Figura 5.32, presenta un

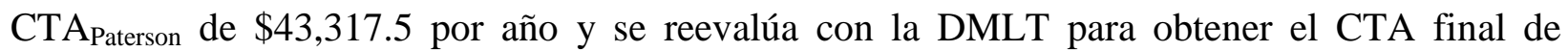
$\$ 43,317.97$. Esta solución aparece por primera vez en la iteración 3291, lo que reafirma, el gran riesgo de realizar pocas iteraciones que no revelen óptimos locales con CTA competitivo.

La red de intercambio de calor determinada en este trabajo no solo presenta un CTA más económico que las soluciones determinadas por otros autores, sino que también exhibe una estructura de red más simple pues únicamente requiere dos divisores de corriente y cuatro subcorrientes en total. Mientras que la solución de Wu y col. (2015) al igual que la desarrollada por Petterson (2008) requieren de tres divisores con seis sub-corrientes en total. Por otro lado, la solución determinada por Lin y Miller (2004) presenta tres divisores y siete sub-corrientes.

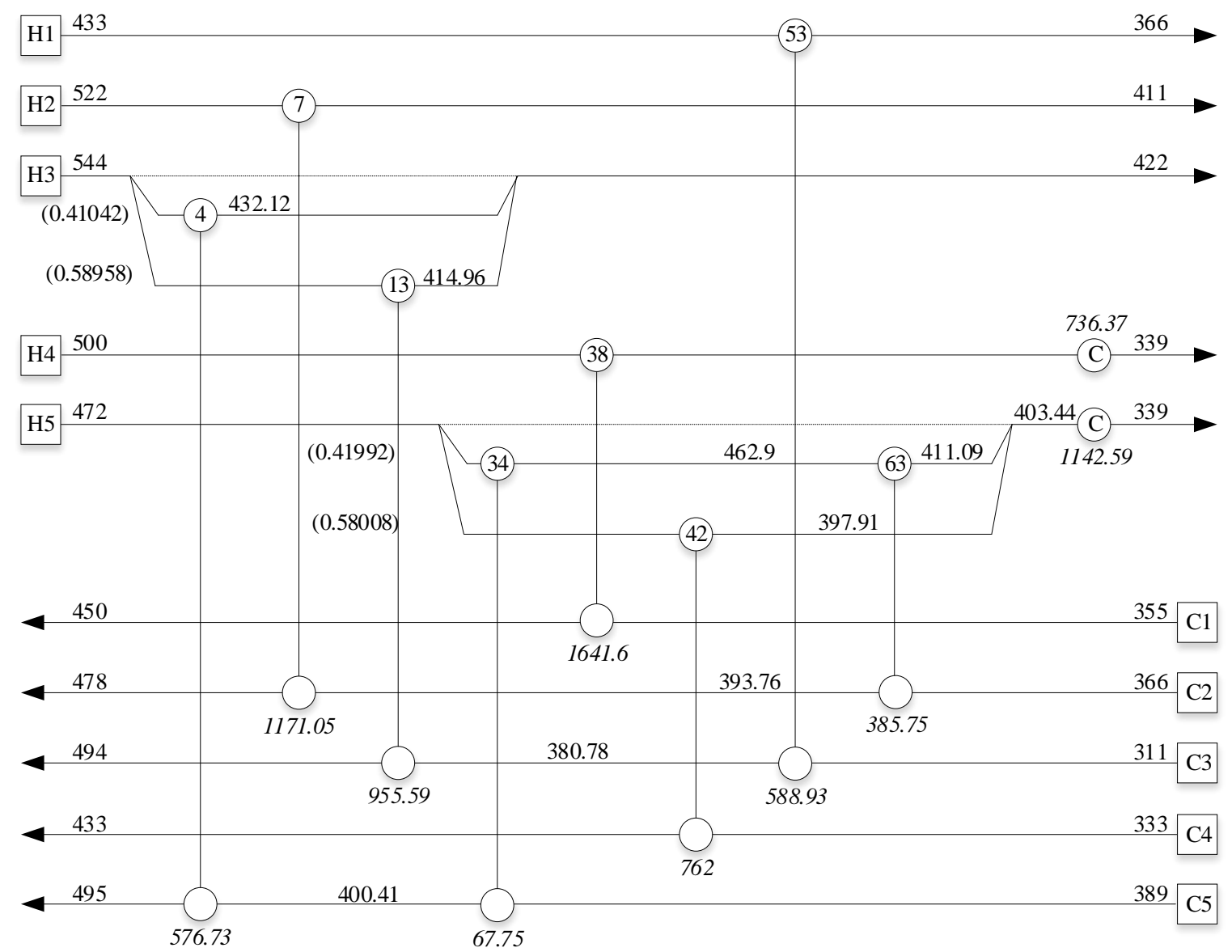

Figura 5.32. Mejor solución óptima local para el Caso de Estudio 5.6 con CTA de \$43,317.97 (temperaturas $(\mathrm{K})$ en fuente normal, cargas térmicas $(\mathrm{kW})$ en cursivas y fracciones de flujo entre paréntesis). 


\section{Comparación de resultados}

En la Tabla 5.31 se muestran los mejores resultados obtenidos por autores que han abordado el Caso de Estudio 5.6 comparados con la mejor solución obtenida en este trabajo.

Tabla 5.31. Comparación del Caso de Estudio 5.6 con soluciones reportadas en la literatura.

\begin{tabular}{|c|c|c|c|}
\hline Autor(es) & Metodología & Configuración ${ }^{1}$ & $\begin{array}{c}\text { CTA Reportado } \\
\$ \text { año }^{-1}\end{array}$ \\
\hline Flower y Linnhoff (1979) & Método ED & $8,0,2-0(0)$ & $43,468.96$ \\
\hline Lewin y Col. (1998) & Algoritmos genéticos & $8,0,2-0(0)$ & $43,751.40$ \\
\hline Lewin (1998) & Algoritmos genéticos & $8,0,2-0(0)$ & $43,798.44$ \\
\hline Chakraborty and Ghosh (1999) & Aleatorización & $8,0,2-0(0)$ & $44,521.41$ \\
\hline Lin y Miller $(2004)^{2}$ & $\begin{array}{l}\text { Algoritmo de } \\
\text { búsqueda tabú }\end{array}$ & $\begin{array}{l}8,0,2-3(7) \\
8,0,2-3(6) \\
8,0,2-4(8)\end{array}$ & $\begin{array}{c}43,329.24 \\
43,350 \\
43,357\end{array}$ \\
\hline Pariyani y col. (2006) & Aleatorización & $8,0,2-0(0)$ & $43,439.69$ \\
\hline Bergamini y Col. (2007) & $\begin{array}{l}\text { Optimización global } \\
\text { Superestructura }\end{array}$ & $8,0,2-0(0)$ & $43,584.15^{*}$ \\
\hline Petterson (2008) & $\begin{array}{l}\text { Optimización Global- } \\
\text { Superestructura }\end{array}$ & $8,0,2-3(6)$ & $43,330.94$ \\
\hline Yerramsetty y Murty (2008) & Evolución diferencial & $8,0,2-0(0)$ & $43,536.07 * *$ \\
\hline Gupta y Ghosh (2010) & Aleatorización & $8,0,2-1(2)$ & $43,342.46$ \\
\hline Huang and Karimi (2013) & Superestructura & $8,0,2-1(2)$ & $43,359.33$ \\
\hline Wu y Col. $(2015)^{2}$ & $\begin{array}{l}\text { Modelo de mezclado } \\
\text { no isotérmico }\end{array}$ & $8,0,2-3(6)$ & $43,319.28$ \\
\hline Peng and Cui (2015) & Recocido simulado & $8,0,2-0(0)$ & $43,410.89$ \\
\hline Este trabajo & $\begin{array}{c}\text { Topologías } \\
\text { redundantes }\end{array}$ & $8,0,2-2(4)$ & $43,317.97$ \\
\hline
\end{tabular}

${ }^{1}$ Recuperadores,Calentadores,Enfriadores-No. de Divisores de corrientes(No. de Sub-corrientes).

${ }^{2}$ Solución revisada en el Apéndice A

* CTA reportado con la aproximación de Paterson

**CTA reportado con la aproximación de Chen 


\subsection{Caso de Estudio 5.7}

El Caso de Estudio 5.7 corresponde a un problema de mediana-gran escala que presenta un total de quince corrientes de proceso, introducido a la literatura por Björk y Petterson (2003). Los datos del problema se enlistan en la Tabla 5.32. Los autores proponen una alteración del modelo Synheat para la síntesis de redes de intercambio de calor utilizando un método híbrido que combina un enfoque de búsqueda estocástica de algoritmos genéticos con una técnica de optimización determinista de PNL-EM, reportando un resultado óptimo con $\mathrm{CTA}_{\text {Paterson }}$ de $\$ 1,513,854$. Posteriormente Björk y Nordman (2005) retoman el mencionado modelo, para abordar el problema de reacondicionamiento de redes de intercambio de calor determinando una solución con $\mathrm{CTA}_{\text {Paterson }}$ de $\$ 1,530,063$. En los trabajos anteriores las estructuras óptimas no se encuentran reportadas por los autores.

Fieg y col. (2009) proponen el uso de un algoritmo mono-genético para el diseño óptimo de redes de intercambio de calor de gran escala basado en la optimización de sub-redes obteniendo un diseño final con CTA de $\$ 1,510,891$. Escobar y Trierweiler (2013) por otro lado, abordan el problema haciendo una comparación entre los enfoques clásicos de síntesis secuenciales y simultáneos y proponiendo diferentes estrategias de inicialización. La mejor solución obtenida por dichos autores es desarrollada mediante el modelo Synheat presentando un CTA Chen de $\$ 1,506,667$, siendo la solución que presenta el menor costo reportado en la literatura. Peng y Cui (2015) resuelven el problema usando un método simultáneo basado en el algoritmo de recocido simulado mediante un procedimiento de dos niveles, estos autores obtienen una estructura óptima sin división de corrientes con CTA de \$1,527,240 la cual requirió más de 26 días de CPU. Chen y col. (2017) mediante un algoritmo de evolución diferencial de multi-población basado en la superestructura Synheat sin división de corrientes, desarrollaron una solución con CTA de $\$ 1,523,735$. Wang y col. (2017) proponen un método de síntesis de dos niveles para optimizar las variables enteras y continuas por separado mediante optimización memética de enjambre de partículas, concluyendo en una estructura óptima con CTA de $\$ 1,519,250$. Xiao y Cui (2017) determinan una solución con CTA de \$1,518,968 mediante un algoritmo de caminata aleatoria con evolución compulsiva. Pavão y col. (2017a) presentan un método híbrido para la síntesis de redes de intercambio de calor sin división de corrientes usando recocido simulado para la optimización de la estructura topológica, mientras que las variables continuas son 
optimizadas mediante enjambre de partículas, este enfoque les permite determinar una solución utilizando la aproximación de Chen (Chen, 1987) para la DMLT, con CTA Chen $_{\text {de } \$ 1,525,394 .}$ Recientemente Pavão y col. (2017b) con su enfoque híbrido de recocido simulado y optimización de fuegos artificiales desarrollaron una solución con CTA de \$1,507,290.

Tabla 5.32. Datos de corrientes y costos para el Caso de Estudio 5.7.

\begin{tabular}{|c|c|c|c|c|}
\hline Corriente & $\begin{array}{c}\text { Temperatura de } \\
\text { suministro } \\
\mathrm{T}\left({ }^{\circ} \mathrm{C}\right)\end{array}$ & $\begin{array}{c}\text { Temperatura } \\
\text { objetivo } \\
\mathrm{T}\left({ }^{\circ} \mathrm{C}\right)\end{array}$ & $\begin{array}{c}\text { Flujo de } \\
\text { capacidad } \\
\text { calorífica } \\
\mathrm{F}\left(\mathrm{kW}^{\circ} \mathrm{C}^{-1}\right)\end{array}$ & $\begin{array}{l}\text { Coeficiente de } \\
\text { película } \\
\mathrm{h}\left(\mathrm{kW} \mathrm{m}^{-2}{ }^{\circ} \mathrm{C}^{-1}\right)\end{array}$ \\
\hline H1 & 180 & 75 & 30 & 2 \\
\hline $\mathrm{H} 2$ & 280 & 120 & 60 & 1 \\
\hline H3 & 180 & 75 & 30 & 2 \\
\hline $\mathrm{H} 4$ & 140 & 40 & 30 & 1 \\
\hline $\mathrm{H} 5$ & 220 & 120 & 50 & 1 \\
\hline H6 & 180 & 55 & 35 & 2 \\
\hline $\mathrm{H} 7$ & 200 & 60 & 30 & 0.4 \\
\hline H8 & 120 & 40 & 100 & 0.5 \\
\hline $\mathrm{C} 1$ & 40 & 230 & 20 & 1 \\
\hline $\mathrm{C} 2$ & 100 & 220 & 60 & 1 \\
\hline $\mathrm{C} 3$ & 40 & 190 & 35 & 2 \\
\hline $\mathrm{C} 4$ & 50 & 190 & 30 & 2 \\
\hline $\mathrm{C} 5$ & 50 & 250 & 60 & 2 \\
\hline C6 & 90 & 190 & 50 & 1 \\
\hline C7 & 160 & 250 & 60 & 3 \\
\hline $\mathrm{HU}$ & 325 & 325 & - & 1 \\
\hline $\mathrm{CU}$ & 25 & 40 & - & 2 \\
\hline \multicolumn{5}{|c|}{$\begin{array}{l}\text { Costo de servicios de calentamiento }=80\left(\$ \mathrm{~kW}^{-1} \mathrm{año}^{-1}\right) \\
\text { Costo de servicios de enfriamiento }=10\left(\$ \mathrm{~kW}^{-1} \mathrm{año}^{-1}\right)\end{array}$} \\
\hline
\end{tabular}

\section{Elección de parámetros de entrada}

Los resultados del método de "Super-Targeting" para el Caso de Estudio 5.7 se muestran en la Tabla 5.33, el valor del HRAT estimado como óptimo se sitúa en $12{ }^{\circ} \mathrm{C}$. En la Figura 5.33 se muestra la selección del intervalo de HRAT que comprende desde un $\operatorname{HRAT}^{\mathrm{L}}=11^{\circ} \mathrm{C}$ hasta un 
$\operatorname{HRAT}^{\mathrm{U}}=13{ }^{\circ} \mathrm{C}$ con un tamaño de paso $t=0.1{ }^{\circ} \mathrm{C}$. Se asignan 2000 inicializaciones aleatorias para la optimización de cada diseño base.

Tabla 5.33. Valores estimados por el método de "Super-Targeting" para el Caso de Estudio 5.7.

\begin{tabular}{ccccc}
\hline $\begin{array}{c}\text { HRAT } \\
\left({ }^{\circ} \mathrm{C}\right)\end{array}$ & $\begin{array}{c}\text { Calor } \\
\text { recuperado } \\
\mathrm{q}_{\text {rec }}(\mathrm{kW})\end{array}$ & $\begin{array}{c}\text { Servicios de } \\
\text { calentamiento } \\
\mathrm{qhu}(\mathrm{kW})\end{array}$ & $\begin{array}{c}\text { Servicios de } \\
\text { enfriamiento } \\
\text { qcu }(\mathrm{kW})\end{array}$ & $\begin{array}{c}\text { CTA } \\
\text { estimado } \\
\left(\$ \text { año }^{-1}\right)\end{array}$ \\
\hline 1 & 36245 & 6605 & 4230 & $2,580,843.38$ \\
10 & 33950 & 8900 & 6525 & $1,525,482.41$ \\
11 & 33695 & 9155 & 6780 & $1,518,430.67$ \\
$\mathbf{1 2}$ & $\mathbf{3 3 4 4 0}$ & $\mathbf{9 4 1 0}$ & $\mathbf{7 0 3 5}$ & $\mathbf{1 , 5 1 5 , 2 8 7 . 4 6}$ \\
13 & 33185 & 9665 & 7290 & $1,515,293.90$ \\
20 & 31400 & 11450 & 9075 & $\mathbf{1 , 5 6 8 , 9 9 0 . 0 8}$ \\
\hline
\end{tabular}

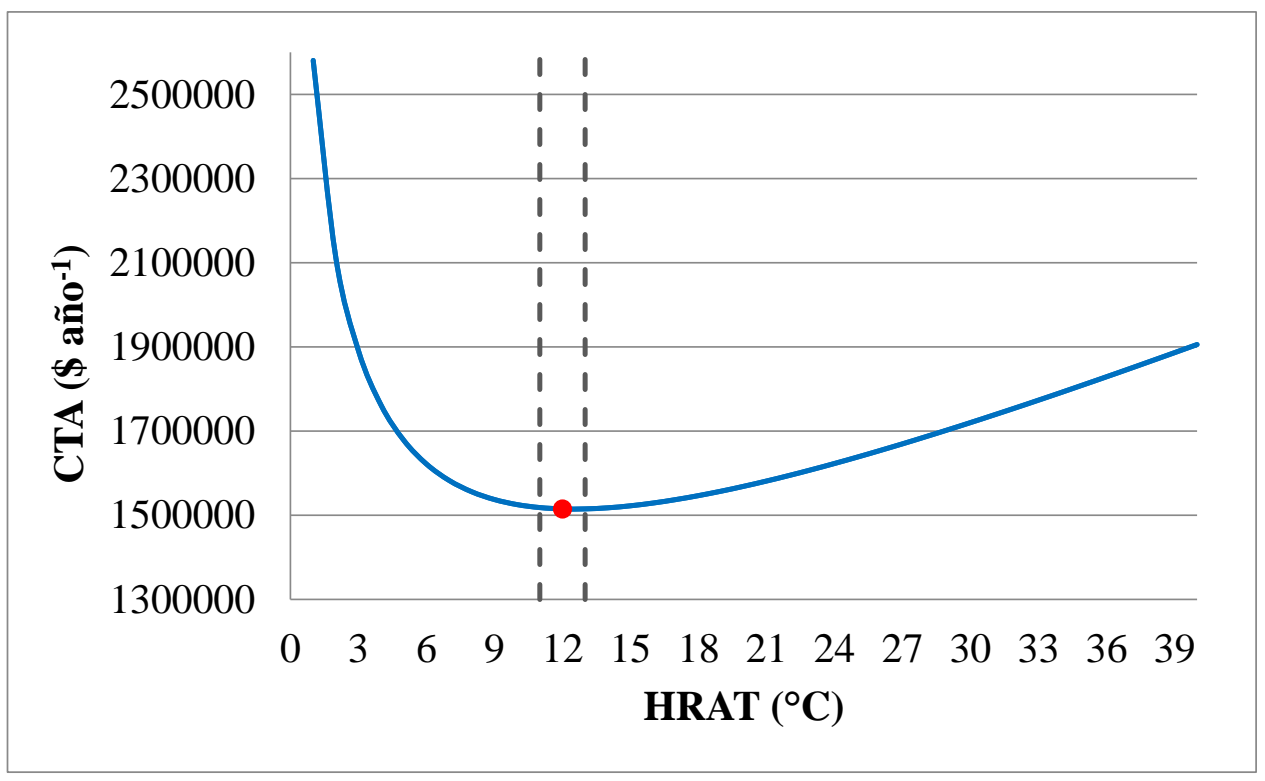

Figura 5.33. "Super-Targeting” y selección del intervalo de HRAT para la generación de topologías redundantes para el Caso de Estudio 5.7.

\section{Generación y optimización de topologías redundantes}

En la Tabla 5.34 se presenta el resumen de resultados del caso de estudio abordado. Como era de esperarse, un problema en donde se presenta un mayor número de corrientes de proceso también involucra un incremento considerable en los equipos de intercambio de calor en cada nivel de 
redundancia. Lo anterior se traduce en un mayor requerimiento de tiempo de cómputo dedicado a cada inicialización para optimizar las estructuras redundantes generadas. Por otro lado, a diferencia de los casos de estudio resueltos anteriormente, en la Tabla 5.34 se puede distinguir que los óptimos locales diferentes en los primeros dos niveles de redundancia representan casi la mitad del total de las soluciones factibles determinadas para cada nivel respectivamente, esto pudiera significar que la complejidad del problema se ha incrementado considerablemente por lo que el enfoque de optimización difícilmente determina soluciones repetidas partiendo de los diferentes puntos de arranque aleatorios, esto implica que es necesario realizar una exploración profunda de topología redundante, buscando reducir la incertidumbre en la posible omisión de óptimos locales a cambio una inversión importante de recursos computacionales.

Tabla 5.34. Resultados del Caso de Estudio 5.7.

Nivel de redundancia Primer nivel Segundo nivel Tercer nivel

\begin{tabular}{lccc}
\hline Topologías redundantes generadas & 21 & 21 & 21 \\
Número de inicializaciones por diseño base & 2000 & 2000 & 2000 \\
Soluciones factibles determinadas & 37949 & 39149 & 40201 \\
Óptimos locales diferentes & 14771 & 16886 & 13414 \\
Promedio de equipos en topologías redundantes & 98 & 79 & 54 \\
Promedio de equipos en diseños óptimos & 19 & 20 & 18 \\
Tiempo de CPU promedio por inicialización & $0.2102 \mathrm{~s}$ & $0.15377 \mathrm{~s}$ & $0.08622 \mathrm{~s}$ \\
Tiempo total de CPU & $2.452 \mathrm{hrs}$ & $1.794 \mathrm{hrs}$ & $1.006 \mathrm{hrs}$ \\
\hline
\end{tabular}

En la Figura 5.34 se muestra el mejor óptimo local de cada topología redundante generada mediante los tres niveles de redundancia disponibles. La mejor solución es determinada utilizando un HRAT de $12.7^{\circ} \mathrm{C}$ en el tercer nivel de redundancia, presentando un $\mathrm{CTA}_{\text {Paterson }}$ de $\$ 1,552,767$. La solución de menor costo requiere de 19 intercambiadores de calor de los cuales dos son enfriadores presentes en las corrientes de proceso $\mathrm{H} 4$ y H8, y siete calentadores son requeridos en las corrientes frías C1, C2, C3, C4, C5, C7 y C8. Dicha solución exhibe un costo por arriba del promedio en comparación con otras soluciones reportadas en la literatura, por lo que para este problema en particular es necesario llevar al límite las capacidades del método propuesto para generar y optimizar un número considerable de diseños iniciales que incrementen las posibilidades de determinar diseños de red finales con costos competitivos. 


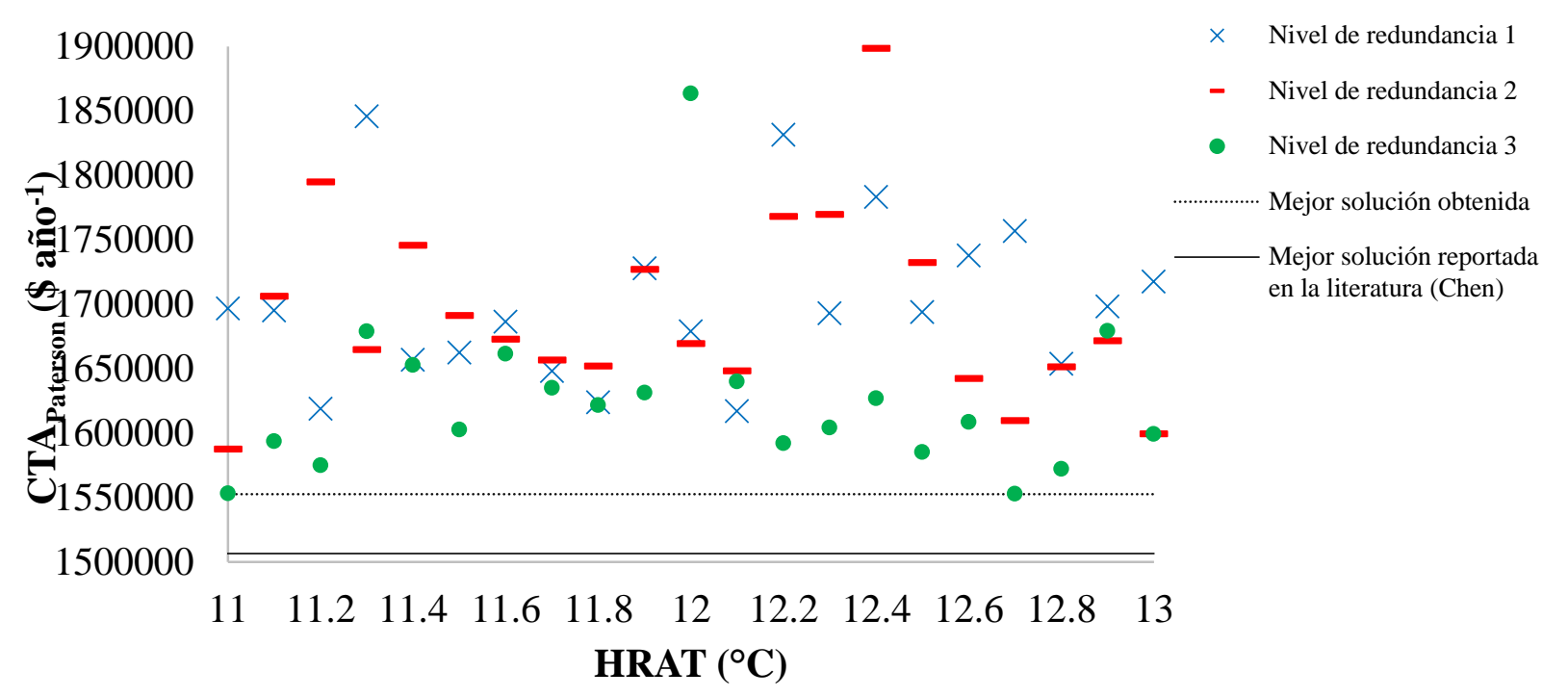

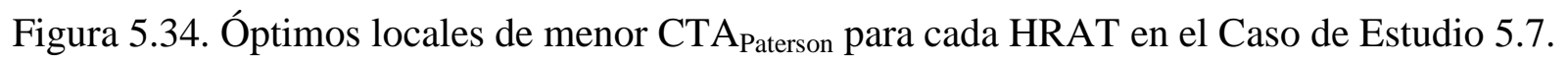

\section{Generación y optimización de topologías redundantes restringiendo servicios auxiliares}

Se busca incrementar las posibilidades de obtener soluciones con costo competitivo impidiéndole al método de optimización caer en óptimos locales con uso excesivo de servicios auxiliares prohibiendo el uso de servicios que no fueron requeridos en la mejor solución obtenida anteriormente. Para esto se imponen costos de transporte altos asociados a las corrientes C6, H1, $\mathrm{H} 2, \mathrm{H} 3, \mathrm{H} 5, \mathrm{H} 6$ y $\mathrm{H} 7$ fijando los parámetros $C H U_{6, l 1}=M, C C U_{1, l}=M, C C U_{2, l}=M$, $C C U_{3, l}=M, C C U_{5, l}=M, C C U_{6, l}=M$ y $C C U_{7, l}=M$. Adicionalmente se plantea diversificar e intensificar la búsqueda fijando un incremento en el HRAT de $t=0.01{ }^{\circ} \mathrm{C}$ y aumentando a 5000 las inicializaciones por cada diseño base generado, utilizando el primer nivel de redundancia. En la Tabla 5.35 se describen los resultados de la prueba realizada prohibiendo servicios auxiliares. Es importante mencionar que para este caso de estudio se generaron 201 diseños iniciales al igual que para el Caso de Estudio 5.7 presentado en la Sección 5.6, sin embargo, debido al incremento en el número de equipos redundantes, el tiempo de cómputo total destinado a la optimización de los diseños base generados se ha incrementado más de cinco veces para este problema. Esto sugiere que en problemas de escala industrial pudiera resultar poco eficiente diseñar redes de intercambio de calor usando el primer nivel de redundancia a menos de que el tiempo de cómputo no resulte una limitante para el diseñador. 
Tabla 5.35. Resultados del Caso de Estudio 5.7 prohibiendo servicios auxiliares a las corrientes de proceso e intensificando la búsqueda.

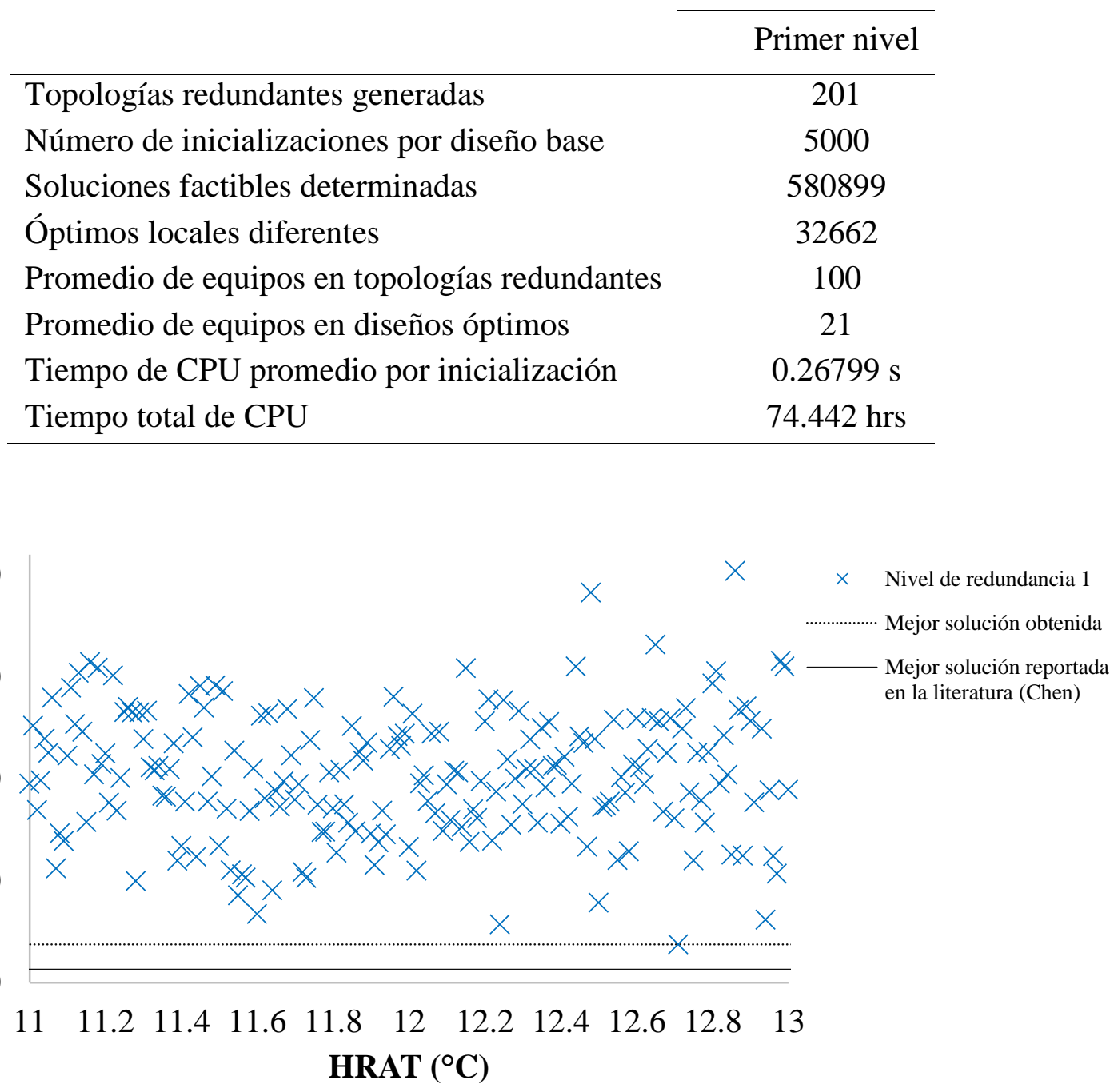

Figura 5.35. Búsqueda intensiva de soluciones óptimas locales para cada HRAT en el Caso de Estudio 5.7, prohibiendo los servicios auxiliares a las corrientes de proceso C6, H1, H2, H3, H5, H6 y H7.

En la Figura 5.35 se muestran los óptimos locales de menor costo para la prueba con servicios auxiliares prohibidos. Mediante esta formulación se determina una solución mediante el HRAT de $12.7{ }^{\circ} \mathrm{C}$ que presenta un $\mathrm{CTA}_{\text {Paterson }}$ de $\$ 1,518,788$ la cual se reevalúa con la DMLT alcanzando un CTA final de $\$ 1,519,049.24$, esta solución se encuentra en el promedio de las soluciones reportadas en la literatura. La topología redundante de 103 intercambiadores de calor que contiene la mejor solución se muestra en la Figura 5.36. Esta estructura plantea 7323 variables y 6984 restricciones para su formulación en el modelo de PNL. 
El diseño simplificado de la solución óptima local de menor costo se presenta en la Figura 5.37 en la cual se han eliminado la mayoría de los equipos permaneciendo únicamente diez recuperadores, cuatro calentadores y dos enfriadores, otorgando un total de 16 intercambiadores de calor. La solución obtenida en este trabajo presenta un equipo menos que la mejor solución reportada en la literatura (Escobar y Trierweiler, 2013) y tres equipos menos que la segunda mejor solución (Pavão y col., 2017b).

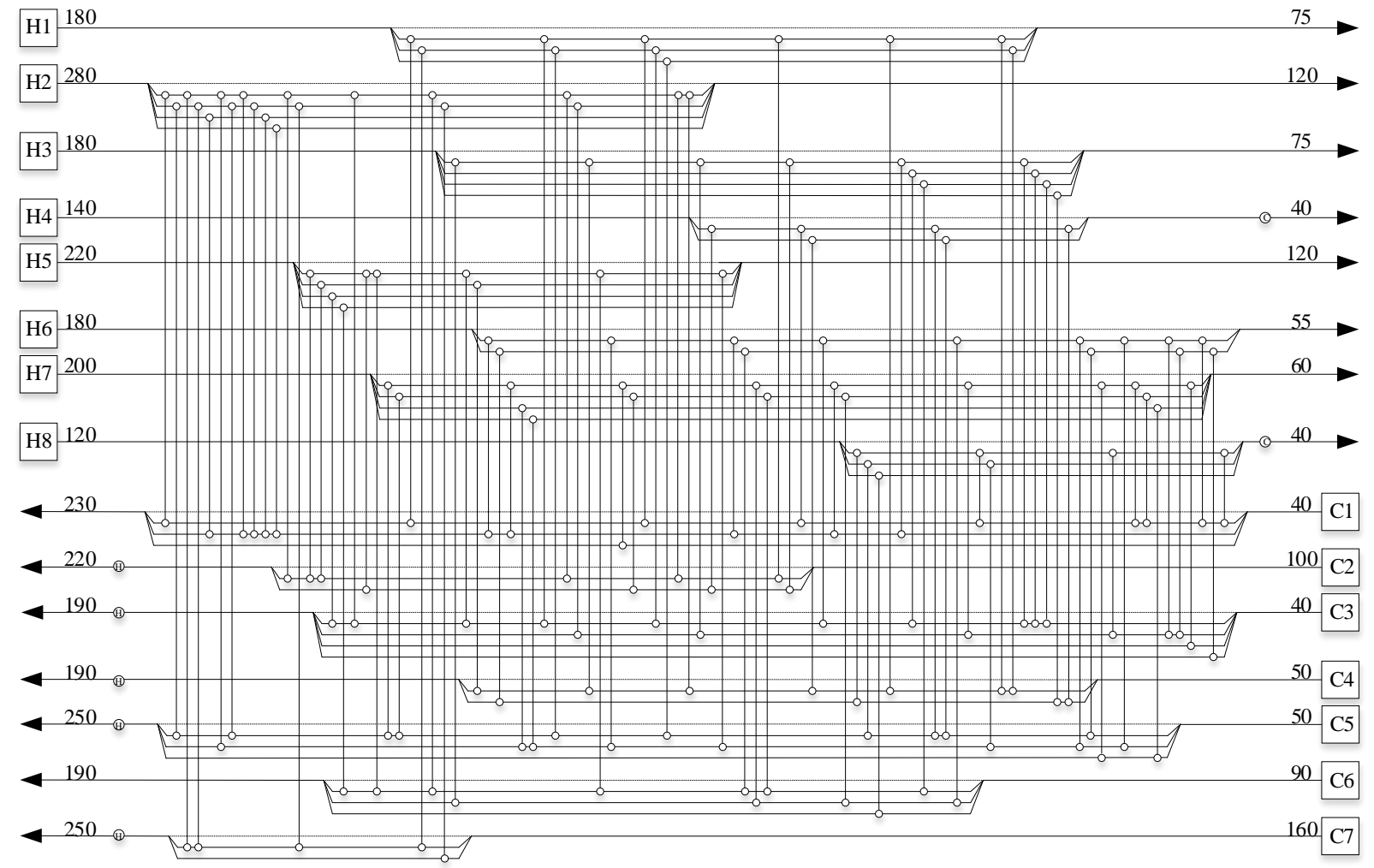

Figura 5.36. Topología redundante generada con un HRAT de $12.71{ }^{\circ} \mathrm{C}$ y una búsqueda intensiva usando el primer nivel de redundancia para el Caso de Estudio 5.7. 


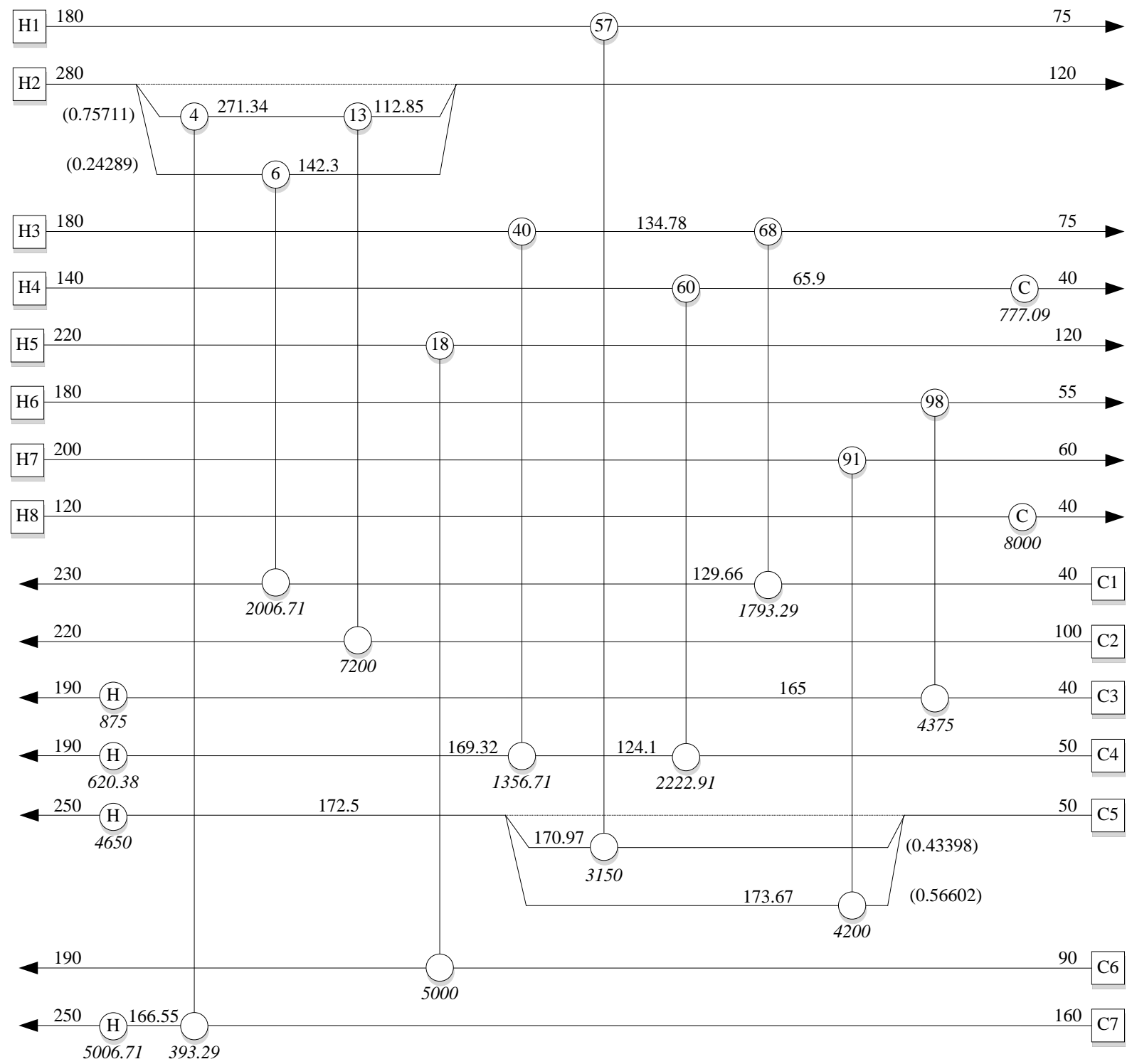

Figura 5.37. Mejor solución óptima local para el Caso de Estudio 5.7 con CTA de \$1,519,049.24 (temperaturas $\left({ }^{\circ} \mathrm{C}\right)$ en fuente normal, cargas térmicas $(\mathrm{kW})$ en cursivas y fracciones de flujo entre paréntesis).

\section{Comparación de resultados}

En la Tabla 5.36 se detalla una comparación de la solución obtenida con la metodología propuesta en este trabajo, contra otras soluciones reportadas en la literatura.

Tabla 5.36. Comparación del Caso de Estudio 5.7 con soluciones reportadas en la literatura. 


\begin{tabular}{|c|c|c|c|}
\hline Autor(es) & Metodología & Configuración ${ }^{1}$ & $\begin{array}{c}\text { CTA Reportado } \\
\$ \text { año }\end{array}$ \\
\hline Björk y Petterson (2003) & $\begin{array}{c}\text { Algoritmos Genéticos; } \\
\text { PNL-EM }\end{array}$ & - & $1,513,854^{*}$ \\
\hline Björk y Nordman (2005) & $\begin{array}{c}\text { Algoritmos Genéticos; } \\
\text { PNL-EM }\end{array}$ & - & $1,530,063 *$ \\
\hline Fieg y col. (2009) & $\begin{array}{l}\text { Algoritmo mono- } \\
\text { genético }\end{array}$ & $9,4,2-1(2)$ & $1,510,891$ \\
\hline Escobar y Trierweiler $(2013)^{2}$ & Synheat & $11,4,2-1(2)$ & $1,506,667 * *$ \\
\hline Peng y Cui (2015) & Recocido simulado & $11,5,3-0(0)$ & $1,527,240$ \\
\hline Chen y col. (2017) & $\begin{array}{l}\text { Evolución diferencial- } \\
\text { Synheat }\end{array}$ & $10,6,3-0(0)$ & $1,523,725$ \\
\hline Wang y col. (2017) & Enjambre de partículas & $10,6,3-0(0)$ & $1,519,250$ \\
\hline Xiao y Cui (2017) & $\begin{array}{l}\text { Caminata aleatoria- } \\
\text { evolución compulsiva }\end{array}$ & $10,6,3-0(0)$ & $1,518,968$ \\
\hline Pavão y col. (2017a) & $\begin{array}{l}\text { Recocido simulado- } \\
\text { enjambre de partículas }\end{array}$ & $10,6,3-0(0)$ & $1,525,394 * *$ \\
\hline Pavão y col. (2017b) & $\begin{array}{l}\text { Recocido simulado- } \\
\text { fuegos artificiales }\end{array}$ & $11,5,3-1(2)$ & $1,507,290$ \\
\hline Este trabajo & $\begin{array}{c}\text { Topologías } \\
\text { redundantes }\end{array}$ & $10,4,2-2(4)$ & $1,519,049.24$ \\
\hline
\end{tabular}

\footnotetext{
${ }^{1}$ Recuperadores,Calentadores,Enfriadores-No. de Divisores de corrientes(No. de Sub-corrientes).

${ }^{2}$ Solución revisada en el Apéndice A

* CTA reportado con la aproximación de Paterson

**CTA reportado con la aproximación de Chen
} 


\subsection{Resumen de resultados}

En esta sección se muestran de forma sintetizada los mejores resultados obtenidos mediante la metodología de síntesis secuencial de redes de intercambio de calor propuesta en este trabajo que consiste en la generación y optimización de topologías redundantes. Los diseños de red reportados en la literatura que presentan el menor CTA, así como los determinados en este trabajo, se revisaron y evaluaron con la DMLT en el Apéndice A, para poder realizar una comparación directa entre las soluciones.

En la Tabla 5.37 se contrastan las características de los diseños de cada problema, que representan a la mejor solución obtenida comparada con la mejor solución reportada en la literatura hasta el momento. En el primer bloque de la tabla ubicado del lado izquierdo, se indica el caso de estudio y el número de corrientes calientes y frías que caracterizan a cada problema. Sobre el segundo bloque se describen las soluciones reportadas en la literatura que presentan el costo más bajo, así como la configuración estructural que presentan. En la tercera columna se detallan los mejores resultados obtenidos en este trabajo para cada caso de estudio. En los Casos de Estudio 5.1, 5.4 y 5.6 fue posible determinar soluciones que presentan mejoras en el costo en comparación con las reportadas por otros autores.

Tanto para el Caso de Estudio 5.2 y 5.3 las soluciones desarrolladas en este trabajo presentan estructuras similares a las mejores soluciones conocidas, con la diferencia de que la metodología propuesta en este trabajo no admite servicios auxiliares intermedios, que para estas soluciones en particular permiten disminuir el costo del diseño final. Por otro lado, la metodología propuesta en este trabajo es capaz de obtener las soluciones para estos problemas en tiempos de cómputo razonables, siendo una ventaja en comparación a las formulaciones propuestas por los autores que abordan estos problemas.

En los peores escenarios como son el Caso de Estudio 5.5 y 5.7, fue posible obtener soluciones que presentan una diferencia considerablemente pequeña en comparación a las mejores soluciones reportadas en la literatura, presentando diseños con un CTA menor al 1\% por arriba del costo determinado por otros autores. No obstante, características destacables surgen de estas soluciones, como pudieran ser estructuras de red más simples, con un número menor de intercambiadores de calor o menos divisiones de corrientes. 
Tabla 5.37. Resumen de resultados para los siete casos de estudio abordados en este trabajo.

\begin{tabular}{|c|c|c|c|c|c|c|c|c|c|c|}
\hline \multirow[b]{2}{*}{$\begin{array}{c}\text { Caso } \\
\text { de } \\
\text { estudio }\end{array}$} & \multirow[b]{2}{*}{ Corrientes } & \multicolumn{3}{|c|}{ Mejor solución reportada en la literatura } & \multicolumn{6}{|c|}{ Este trabajo } \\
\hline & & Autor(es) & Configuración ${ }^{1}$ & $\begin{array}{c}\text { CTA } \\
\text { revisado } \\
\left(\$ \text { año }^{-1}\right)\end{array}$ & $\begin{array}{l}\text { Tiempo } \\
\text { de } \\
\text { CPU total }\end{array}$ & Restricciones & Variables & Configuración $^{1}$ & $\begin{array}{c}\text { CTA } \\
\left(\$ \text { año }^{-1}\right)\end{array}$ & $\begin{array}{c}\text { Diferencia } \\
\%\end{array}$ \\
\hline 5.1 & $2 h, 2 c$ & Björk y Westerlund (2002) & $4,1,1-0(0)$ & $415,445.24$ & $2.957 \mathrm{~min}$ & 187 & 229 & $3,1,1-1(2)$ & $411,624.48$ & -0.92 \\
\hline 5.2 & $3 \mathrm{~h}, 2 \mathrm{c}$ & Gupta y Ghosh (2010) & $3,1,1-1(2)$ & $80,378.98$ & $10.46 \mathrm{~min}$ & 468 & 540 & $3,1,1-1(2)$ & $80,847.71$ & +0.58 \\
\hline 5.3 & $4 \mathrm{~h}, 3 \mathrm{c}$ & Huang y Karimi (2013) & $5,1,2-1(2)$ & $105,421.83$ & $3.24 \mathrm{hrs}$ & 1284 & 1424 & $5,1,2-1(2)$ & $105,481.12$ & +0.06 \\
\hline 5.4 & $4 \mathrm{~h}, 4 \mathrm{c}$ & Li y col. (2004) & $6,2,0-2(4)$ & $29,326.00$ & $50.86 \mathrm{~min}$ & 1557 & 1700 & $7,1,0-2(4)$ & $29,156.65$ & -0.58 \\
\hline 5.5 & $4 h-5 c$ & Petterson (2005) & $11,3,3-7(14)$ & $2,904,953.09$ & $14.15 \mathrm{hrs}$ & 1666 & 1814 & $8,1,3-2(4)$ & $2,919,687.66$ & +0.5 \\
\hline 5.6 & $5 h-5 c$ & Wu y Col. (2015) & $8,0,2-3(6)$ & $43,319.28$ & $10.21 \mathrm{hrs}$ & 4463 & 4743 & $8,0,2-2(4)$ & 43,317.97 & -0.003 \\
\hline 5.7 & $8 \mathrm{~h}-7 \mathrm{c}$ & Escobar y Trierweiler (2013) & $11,4,2-1(2)$ & $1,505,262.18$ & $74.442 \mathrm{hrs}$ & 6984 & 7323 & $10,4,2-2(4)$ & $1,519,049.24$ & +0.91 \\
\hline
\end{tabular}

${ }^{1}$ Recuperadores,Calentadores,Enfriadores-No. de Divisores de corrientes(No. de Sub-corrientes). 


\section{Capítulo 6. Conclusiones y trabajo a futuro}

\subsection{Recapitulación}

En este trabajo se desarrolló una metodología para la síntesis de redes de intercambio de calor que resuelve secuencialmente sub-problemas de generación (PL) y optimización (PNL) de topologías redundantes. La propuesta se basa en la construcción de diseños de red intencionalmente dotados de suficientes grados de libertad, que le permitan a la técnica de optimización de multi-arranque estocástico en dos fases (Núñez-Serna y Zamora, 2016) asignar valores a las variables de decisión como son cargas térmicas, fracciones de flujos, temperaturas intermedias y áreas de intercambio de calor, que minimicen el costo total anual de la red redundante para realizar las debidas reducciones estructurales, eliminando equipos y subcorrientes innecesarias para determinar, dentro de lo posible, los mejores diseños óptimos contenidos en los diseños base.

A diferencia de otras metodologías de los enfoques de síntesis clásicos, tanto secuenciales como simultáneos, no se recurre al uso de técnicas de programación entera mixta, evitando la explosión combinatoria característica de dichas formulaciones, teniendo en su lugar espacios de solución acotados por las estructuras con diseño preestablecido. Para la metodología propuesta, un incremento en el número de corrientes implica también que se destinará un tiempo mayor a cada inicialización realizada, lo que podría suponer un uso de recursos computacionales considerables en problemas de escala industrial, no obstante, esta limitante es impuesta por el criterio de calidad que el diseñador requiera de la solución.

El método fue aplicado satisfactoriamente a siete casos de estudio de referencia, que incluyen desde 4 corrientes de proceso el más pequeño hasta 15 corrientes de proceso el más grande, mostrando en cada caso, diseños de red óptimos con costos competitivos en comparación con las soluciones reportadas en la literatura. En este capítulo se resaltan las contribuciones del enfoque 
propuesto, así como los puntos clave en donde es posible implementar posibles mejoras para incrementar la eficiencia del método.

\subsection{Conclusiones}

Se ha desarrollado una metodología secuencial de síntesis de redes de intercambio de calor recurriendo a nociones de programación matemática y la creación de algoritmos. La propuesta se integra de dos elementos fundamentales. En primer lugar, la generación de las redes de intercambio de calor redundantes, es decir, diseños de red que fueron construidos con la premisa de disponer de un número sobreestimado de equipos, divisiones de corrientes y en algunos casos re-apareamientos de las mismas corrientes de proceso en más de una ocasión. En segundo lugar, la optimización de dichas estructuras mediante la técnica de multi-arranque estocástico en dos fases, que permite hacer una exploración razonable sobre un espacio de solución definido, inicializando las variables de decisión con valores asignados de forma pseudo-aleatorios entre los valores de cotas previamente calculadas. A continuación, se describen brevemente algunos puntos destacables de la metodología propuesta.

\section{Generación de topologías redundantes}

Uno de los principales retos de este trabajo fue formular un método sistemático que permitiera determinar diseños base redundantes y a su vez cumpliera con características que asegurasen la factibilidad del dominio de solución y brindaran compatibilidad con la técnica de optimización. Por estas razones una propuesta de síntesis de redes de intercambio de calor redundantes tuvo que ser elaborada desde la raíz.

En el Capítulo 2 se introduce el problema de uso mínimo de servicios auxiliares como un modelo de transporte (Cerdá y col., 1983). Este modelo de PL asigna rutas de intercambio de calor prometedoras entre corrientes de proceso, necesarias para cumplir con las metas de máxima recuperación de energía. La solución del modelo de transporte no tiene como objetivo determinar redes de costo óptimo, pero otorga intercambios de calor factibles, por lo que si se considera 
estos apareamientos como intercambiadores de calor en potencia es posible interpretarlos como diseños de red que posean un número sobreestimado de equipos.

En el Capítulo 4 se retoma la formulación del modelo de transporte, y se propone interpretar los apareamientos asignados por la solución del modelo de transporte como equipos de intercambio de calor en un diagrama de malla. La dificultad de traducir la distribución de cargas térmicas otorgada por la solución del modelo de transporte en estructuras redundantes surge debido a que los apareamientos entre corrientes de proceso no sugieren una estructura evidente para el diseñador, ni el orden en que los equipos deben ser colocados. En este trabajo se propusieron tres formulaciones para definir diferentes niveles de redundancia en los diseños base, es decir, que el número total de elementos disponibles en la tabla de distribución de cargas térmicas puede ser reducido, integrando apareamientos que cumplan con ciertas reglas heurísticas desarrolladas, en cuyo caso se interpretan como un solo intercambiador de calor. Lo anterior permite disponer de topologías redundantes con diferentes tamaños definidos previamente al proceso de optimización, dando lugar a la generación de diseños base con diferentes características, como son el número y ordenamiento de equipos y las divisiones de corrientes disponibles, lo que pudieran significar la determinación de diferentes óptimos locales y adicionalmente pueden presentar ventajas en términos de inversión de recursos computacionales. El primer nivel posee una mayor cantidad de equipos, disminuyendo consecutivamente el número de éstos en los niveles de redundancia dos y tres.

Una limitante de la metodología propuesta es que solo es posible obtener diseños óptimos contenidos en las topologías redundantes generadas, para superar esto se aprovecharon las características sistemáticas del método proponiendo optimizar un conjunto de diseños base mediante incrementos equidistantes en el HRAT. Este parámetro define el desplazamiento relativo entre las corrientes de proceso en la tabla de calor donde se plantea el modelo de transporte, permitiendo determinar diferentes distribuciones de cargas térmicas que se traducen en diferentes estructuras redundantes a optimizar. La decisión del número de diseños base definidos recae en el diseñador y en la necesidad que se tenga de la obtención de soluciones de calidad. Proponer una mayor cantidad de topologías redundantes significará en principio, un incremento en la probabilidad de obtener soluciones competitivas, pero pudiera afectar fuertemente el tiempo total de CPU dedicado a optimizar todas estas redes. 


\section{Metodología de optimización}

En el Capítulo 3 se hace una descripción completa del algoritmo de multi-arranque estocástico en dos fases (Núñez-Serna y Zamora, 2016). Para los fines de la metodología desarrollada, esta técnica es adoptada como el segundo sub-problema que se resuelve secuencialmente para la síntesis de redes de intercambio de calor. Una de las principales virtudes del enfoque es que únicamente toma en cuenta los elementos estructurales de un diseño base, por lo que a pesar de haber construido las topologías redundantes mediante un enfoque de uso mínimo de servicios auxiliares sin considerar el costo inicial del diseño, es capaz de llevar a cabo posibles reducciones estructurales al definir en cero las cargas térmicas de los equipos redundantes que no son necesarios, para determinar en conjunto los mejores valores de fracciones de flujo, temperaturas intermedias y áreas de intercambio de calor que concluyan en una tarea óptima de recuperación de calor para determinar soluciones finales de bajo costo.

Otra característica que presenta la técnica de optimización es la disminución de las probabilidades de obtener un solo óptimo local de baja calidad, mediante la inicialización pseudo-aleatoria con distribución probabilística uniforme de las variables de decisión, permitiendo explorar diferentes zonas del espacio de solución. No obstante, el número de inicializaciones no sólo está ligado al posible incremento en la calidad de las soluciones, sino también con el tiempo de cómputo destinado a la optimización de una estructura redundante. Por otro lado, esta técnica ha probado tener la capacidad de lidiar con los diseños iniciales propuestos a pesar de incluir un número considerable de variables y restricciones, permitiendo optimizar topologías redundantes de más de cien equipos, y definir soluciones simplificadas con un número óptimo de intercambiadores de calor, y en el mejor de los casos que presentan un costo total anual competitivo.

\section{Resultados obtenidos}

En el Capítulo 5 se detallan los resultados obtenidos para siete casos de estudio abordados, donde cada uno presenta diferencias en el número total de corrientes de proceso, y consideran diferentes funciones de costos. 
A lo largo del desarrollo de este trabajo se identificó que la metodología de optimización en muchas ocasiones no elimina de forma eficiente algunos servicios auxiliares, pudiendo conducir a la determinación de óptimos locales indeseables con costos elevados utilizando recursos que pudieran ser destinados a la identificación de soluciones con mejores probabilidades de presentar costos atractivos en términos de costos. Tomando esto en cuenta, se propuso realizar una primera prueba para registrar los servicios auxiliares que no son requeridos considerando únicamente la mejor solución obtenida y pruebas posteriores donde se prohíben dichos apareamientos sobre la formulación del modelo de transporte con el objetivo de generar diseños iniciales que no contengan los servicios auxiliares indeseados. Esto, en conjunto con una mayor inversión de recursos computacionales al incrementar el número de inicializaciones del método de optimización, permitieron obtener estructuras óptimas con costos competitivos en comparación con las soluciones reportadas en la literatura.

El Caso de Estudio 5.1 es un problema de pequeña escala que comprende dos corrientes de proceso calientes y dos frías. El problema fue abordado autores con técnicas de optimización global determinista. Björk y Westerlund (2002) afirman determinar el óptimo global para este problema mostrando una solución con CTA revisado de $\$ 415,445.24$. A pesar de esto, la solución definida con la metodología propuesta en este trabajo presenta un CTA final correspondiente a $\$ 411,624.48$, lo que significa una mejora del $0.92 \%$ con respecto a la mejor solución reportada en la literatura.

El Caso de Estudio 5.2 presenta tres corrientes calientes y dos corrientes frías. En este problema solución Gupta y Ghosh (2010) determinan una solución con CTA revisado de \$80,378.98. Mediante el enfoque de síntesis desarrollado en este trabajo, fue posible obtener una solución con CTA de $\$ 80,847.71$ la cual se encuentra $0.58 \%$ por encima de la mejor solución reportada, no obstante, ambos diseños presentan estructuras muy similares, donde la diferencia en costos se debe a que la metodología de Gupta y Ghosh (2010) admite colocar servicios intermedios. Esta característica, permite para este caso, una disminución considerable en el CTA del diseño de red.

El Caso de Estudio 5.3 involucra cuatro corrientes de proceso calientes y tres corrientes frías. La solución determinada en este trabajo con CTA de 105,481.12 se encuentra un $0.06 \%$ por arriba del costo de la mejor solución reportada en la literatura desarrollada por Huang con CTA 
revisado de $\$ 105,421.83$. Al igual que en el Caso de Estudio 5.2 ambas estructuras se diferencian únicamente por un servicio auxiliar intermedio colocado sobre una sub-corriente de proceso.

Para el caso de Estudio 5.4 corresponde al problema 8SP1 que consiste de un total de cuatro corrientes de proceso calientes y cuatro frías. La solución determinada en este trabajo con CTA $\$ 29,156.65$ representa una mejora del $0.58 \%$ comparada con la mejor solución obtenida previamente por Luo y col. (2004) con CTA revisado de $\$ 29,326$.

El Caso de Estudio 5.5 que dispone de cuatro corrientes calientes y cinco frías, es el problema conocido como 9SP el cual ha sido abordado frecuentemente por diferentes autores. La mejor solución disponible en la literatura fue determinada por Petterson (2005) y presenta un CTA revisado de \$2,904,953.09. La solución obtenida en este trabajo exhibe un CTA de $\$ 2,919,687.66$ encontrándose un $0.5 \%$ por arriba de la mejor solución reportada en la literatura. Sin embargo, la estructura definida en este trabajo presenta ventajas estructurales al ser considerablemente más simple, requiriendo únicamente 12 intercambiadores de calor y 2 divisiones de corriente, mientras que la solución de Petterson (2005) presenta 17 equipos y un total de 7 divisiones de corriente.

El Caso de Estudio 5.6, es el conocido problema 10SP1 el cual es considerado como uno de los principales problemas de referencia en la literatura. Este caso implica cinco corrientes de proceso calientes y cinco frías. La solución definida mediante el enfoque de síntesis de generación y optimización de redes redundantes presenta un CTA de $\$ 43,317.97$ la cual disminuye marginalmente el costo de la solución obtenida por Wu y col. (2015) con CTA revisado de $\$ 43,319.28$ ubicándose un $0.003 \%$ por debajo de esta. Sumado a esto, la solución obtenida en este trabajo muestra una estructura de red más simple con solo 2 divisiones de corriente en comparación con las 3 divisiones de corriente requeridas por la solución de Wu y col. (2015).

El Caso de Estudio 5.8 consiste en la integración térmica de ocho corrientes de proceso calientes y siete frías. El número de corrientes involucradas en este problema perjudica de forma considerable el tiempo de cómputo requerido por la metodología de síntesis propuesta en este trabajo para la detección de óptimos locales de calidad. La mejor solución determinada por el enfoque de síntesis desarrollado en este trabajo presenta un CTA de $\$ 1,519,049.24$, la cual supone un incremento del $0.91 \%$ con respecto a la mejor solución disponible en la literatura 
determinada por Escobar y Trierweiler (2013) la cual presenta un CTA revisado de $\$ 1,505,262.18$.

En todos los casos de estudio abordados fue posible determinar, soluciones que se encuentran a menos del $1 \%$ por encima de las mejores soluciones reportadas en la literatura, en términos del CTA. Lo anterior pudiera ser considerado un criterio que demuestra la efectividad del método frente a otras formulaciones de síntesis, permitiendo obtener soluciones competitivas si la metodología es llevada al límite al explorar un número suficiente de estructuras redundantes.

Como es de suponerse, el primer nivel de redundancia mostró una menor eficiencia en tiempos de CPU en comparación con el segundo y tercer nivel de redundancia en todos los casos, al disponer de un mayor número de equipos a eliminar. Sin embargo, el primer nivel se utilizó como premisa principal de síntesis puesto que hasta este punto se ha considerado como prioridad la calidad de la solución sobre el tiempo de cómputo. Con esto en mente, se admitió que las estructuras con más equipos pueden incrementar la probabilidad de contener soluciones óptimas locales dando mayor oportunidad de determinar soluciones de costo competitivo. El primer paso de una implementación a futuro en problemas de mayor escala pudiera ser considerar los diferentes niveles de redundancia como premisas de síntesis, permitiendo mejoras considerables en los recursos computacionales requeridos.

La metodología de generación y optimización de topologías redundantes permite diseñar redes con división de corrientes, mezclado no isotérmico y la posibilidad de admitir equipos en serie sobre un mismo ramal de una sub-corriente de proceso; esta característica no se incluye en algunas de las metodologías de síntesis clásicas como el modelo Synheat (Yee y Grossmann, 1990), por lo que es posible determinar soluciones que probablemente han sido poco estudiadas. Las soluciones obtenidas en los Casos de Estudio 5.4, 5.6 y 5.7 son claros ejemplos de esta aseveración. En contraparte, ni la metodología de generación de redes redundantes, ni el enfoque de optimización permiten hasta ahora más de un divisor y mezclador por corriente, y debido a consideraciones realizadas con el fin de mantener limitadas las dificultades computacionales, se decidió generar topologías redundantes restringidas a máximo, un servicio auxiliar extremo por cada corriente de proceso, excluyendo la posibilidad de servicios auxiliares intermedios.

Tomar ventaja de los grados de libertad intencionalmente asignados a diseños redundantes definiendo una estructura base a optimizar, constituye un enfoque de síntesis que no había sido 
previamente considerado en la literatura. Esta metodología, representa un primer acercamiento a una nueva alternativa para enfrentar las limitaciones que tienen los enfoques de programación matemática, omitiendo el uso de variables binarias que suelen incrementar exponencialmente el espacio de solución conforme el número de corrientes incrementa y en su lugar se lidia con múltiples espacios de solución redundantes acotados por estructuras factibles previamente definidas.

\subsection{Posibles líneas de investigación en un trabajo a futuro}

Si bien la metodología de síntesis propuesta ha probado tener la capacidad de definir diseños de red competitivos y en ciertas ocasiones con mejoras en el costo con respecto a las soluciones determinadas por otros enfoques de síntesis, existen diversas áreas de implementación que pudieran incrementar la eficacia del método desarrollado. A continuación, se proponen algunos puntos clave que se considera, podrían significar un paso hacia adelante en la evolución de la metodología propuesta.

En recuento, la principal limitante de la formulación de síntesis secuencial desarrollada en este trabajo es el uso de recursos computacionales que incrementa conforme el número de corrientes aumenta y más importante, conforme se requiere una exploración más exhaustiva para determinar soluciones de bajo costo. Para afrontar esta limitación es posible proponer mejoras tanto en la generación y como en la optimización de las topologías redundantes.

a) Se sugiere plantear un modelo de transporte multi-objetivo que permita minimizar el uso de servicios auxiliares y a su vez reduzca el número de calentadores y enfriadores requeridos para llevar a cabo esta tarea. Lo que permitiría en principio, omitir las pruebas para la prohibición de servicios auxiliares. Bajo la misma línea se introduce la idea de considerar un modelo de transporte ponderado donde apareamientos altamente prometedores para la integración térmica son conservados, y se penalizan aquellos que son prescindibles, esto permitiría determinar diseños base manteniendo control sobre el número final de equipos, abriendo la posibilidad de abordar casos de estudio de escala industrial. 
b) En términos del enfoque de optimización se propone buscar la posibilidad de reducir las características aleatorias de la optimización mediante una metodología de búsqueda informada (e.g. búsqueda tabú) que permita explorar diferentes zonas del espacio y que suponga una convergencia menor a óptimos locales ya definidos. Significando una menor incertidumbre acerca de la omisión de soluciones de calidad con una inversión modesta de recursos computacionales.

Por otro lado, incorporar características estructurales que no fueron incluidas en este trabajo pudieran significar una posibilidad incrementada de obtener soluciones de bajo costo. El uso de servicios auxiliares intermedios y sobre los ramales en las sub-corrientes de proceso, incluir corrientes de desviación (baipás), las posibilidades de flujos cruzados han probado, en algunos casos, conducir a soluciones con área óptima de intercambio de calor que se traduce en diseños de menor costo. Por otro lado, la inclusión de estas características pudiera significar un incremento en el gasto de recursos computacionales por lo que es necesario previamente mejorar este aspecto, así como desarrollar una técnica de optimización que admita dichas características. 


\section{Apéndice A}

\section{A.1. Revisión del Caso de Estudio 5.1}

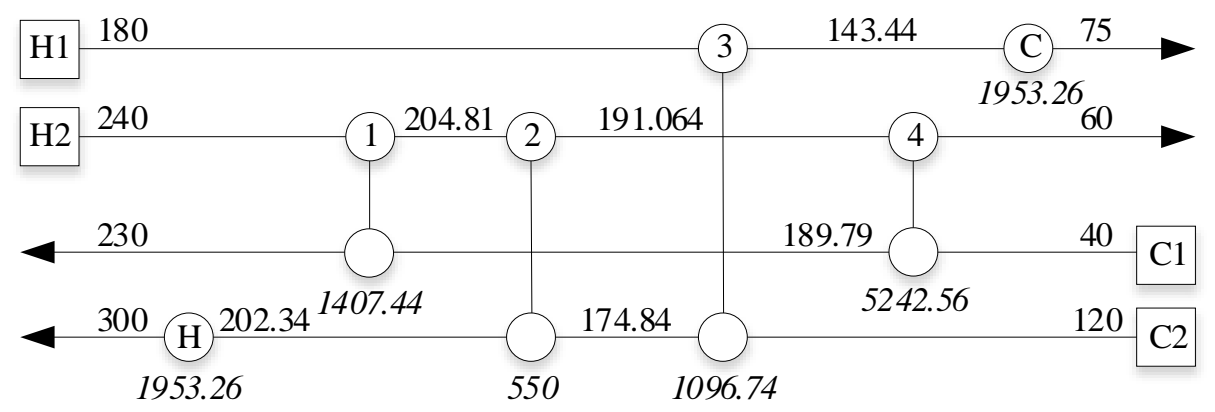

Figura A.1. Diseño de red determinado por Zamora y Grossmann (1997) para el Caso de Estudio 5.1 con $\mathrm{CTA}_{\mathrm{DMLT}}$ reportado de $\$ 419,979$ y $\mathrm{CTA}_{\mathrm{DMLT}}$ revisado de $\$ 419,978.82$.

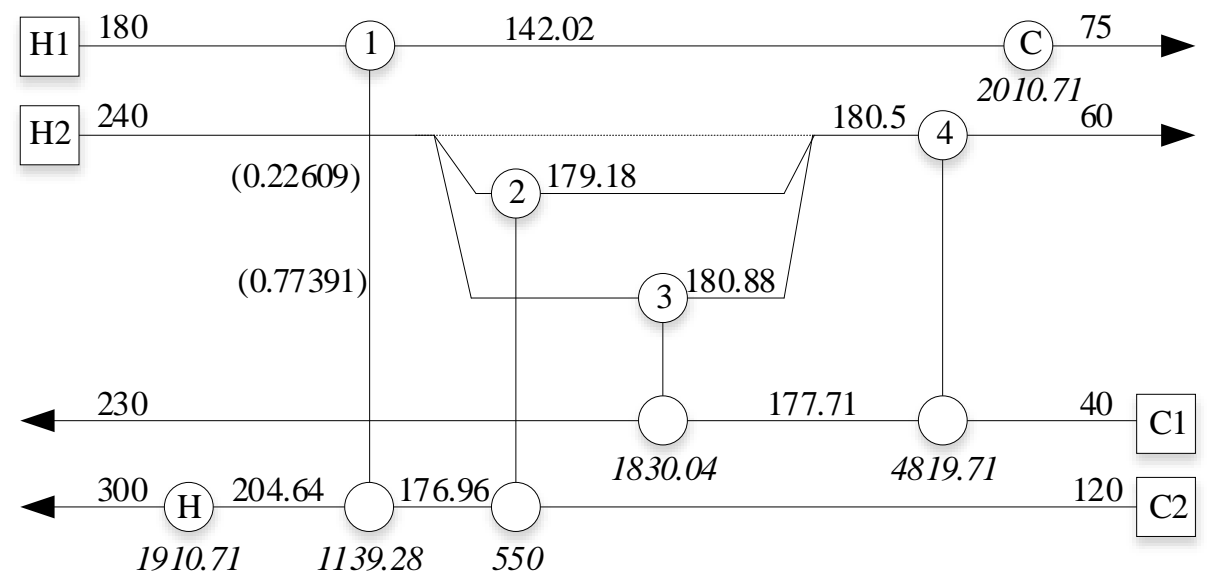

Figura A.2. Diseño de red determinado por Björk y Westerlund (2002) para el Caso de Estudio

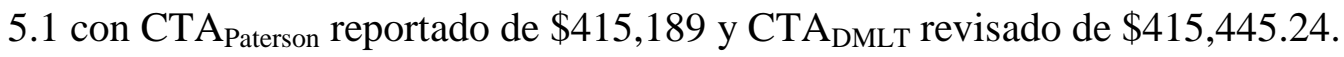


Tabla A.1 Revisión de costos del diseño de red de la Figura A.1 desarrollado por Zamora y Grossmann (1997) para el Caso de Estudio 5.1.

\begin{tabular}{|c|c|c|c|c|c|c|c|c|c|c|c|}
\hline $\begin{array}{l}\text { Número } \\
\text { de ID del } \\
\text { equipo }\end{array}$ & $\begin{array}{l}\text { Corrientes } \\
\text { que } \\
\text { participan }\end{array}$ & $\begin{array}{c}\text { Carga } \\
\text { Térmica } \\
{[\mathrm{kW}]}\end{array}$ & $\frac{\mathrm{hi}}{\left[\mathrm{kWm}^{-2}{ }^{-1} \mathrm{C}^{-1}\right]}$ & $\begin{array}{c}\text { ho } \\
{\left[\mathrm{kWm}^{-2} \mathrm{C}^{-1}\right]}\end{array}$ & $\frac{\mathrm{U}}{\left[\mathrm{kWm}^{-2 \circ} \mathrm{C}^{-1}\right]}$ & $\begin{array}{l}\text { dth } \\
{\left[{ }^{\circ} \mathrm{C}\right]}\end{array}$ & $\begin{array}{l}\mathrm{dtc} \\
{\left[{ }^{\circ} \mathrm{C}\right]}\end{array}$ & $\begin{array}{c}\text { DMLT } \\
{\left[{ }^{\circ} \mathrm{C}\right]}\end{array}$ & $\begin{array}{c}\mathrm{A} \\
{\left[\mathrm{m}^{2}\right]}\end{array}$ & $\begin{array}{l}\text { Costo de capital } \\
{\left[\$ \$ \mathrm{anno}^{-1}\right]}\end{array}$ & $\begin{array}{c}\text { Costo de operación } \\
{\left[\$ \text { \$año }^{-1}\right]}\end{array}$ \\
\hline 1 & $\mathrm{H} 2-\mathrm{C} 1$ & 1407.44 & 0.10 & 0.20 & 0.07 & 10.00 & 15.03 & 12.34 & 1710.39 & 26577.02 & - \\
\hline 2 & $\mathrm{H} 2-\mathrm{C} 2$ & 550.00 & 0.10 & 0.10 & 0.05 & 2.48 & 16.23 & 7.32 & 1503.70 & 25443.61 & - \\
\hline 3 & $\mathrm{H} 1-\mathrm{C} 2$ & 1096.74 & 0.15 & 0.10 & 0.06 & 5.16 & 23.44 & 12.08 & 1513.01 & 25495.30 & - \\
\hline 4 & $\mathrm{H} 2-\mathrm{C} 1$ & 5242.56 & 0.10 & 0.20 & 0.07 & 1.28 & 20.00 & 6.80 & 11556.53 & 68380.56 & \\
\hline HU2 & HU-C2 & 1953.26 & 2.00 & 0.10 & 0.10 & 25.00 & 122.66 & 61.40 & 334.02 & 21268.56 & 110.00 \\
\hline \multirow[t]{6}{*}{$\mathrm{CU} 2$} & H1-CU & 2053.26 & 0.15 & 0.50 & 0.12 & 103.44 & 50.00 & 73.51 & 242.07 & 17422.56 & 10.00 \\
\hline & & & & & \multicolumn{7}{|c|}{15149.33} \\
\hline & & \multicolumn{3}{|c|}{ Calor total recuperado $[\mathrm{kW}]$} & 8296.74 & \multicolumn{5}{|c|}{ Costo total anual de capital $\left[\$ a \tilde{n} o^{-1}\right]$} & 184587.62 \\
\hline & & \multicolumn{3}{|c|}{$\begin{array}{l}\text { Total de servicios de } \\
\text { calentamiento }[\mathrm{kW}]\end{array}$} & 214858.60 & \multicolumn{5}{|c|}{ Costo total anual de operación $\left[\$ a n ̃ o^{-1}\right]$} & 235391.20 \\
\hline & & \multicolumn{3}{|c|}{$\begin{array}{l}\text { Total de servicios de } \\
\text { enfriamiento }[\mathrm{kW}]\end{array}$} & 20532.60 & \multicolumn{5}{|c|}{ Costo total anual de la red $\left[\$ a \tilde{n} o^{-1}\right]$} & $419,978.82$ \\
\hline & & \multicolumn{3}{|c|}{ Área total estimada $\left[\mathrm{m}^{2}\right]$} & 15149.33 & & & & & & \\
\hline
\end{tabular}

\begin{tabular}{cc}
\multicolumn{3}{c}{$\begin{array}{c}\text { Cálculo de temperaturas } \\
\text { intermedias calientes }\end{array}$} \\
th 1,3 & 143.44 \\
\hline th2,1 & 204.81 \\
th 2,2 & 191.06
\end{tabular}

Cálculo de temperaturas intermedias frías

\begin{tabular}{ll}
$\mathrm{tc} 1,4$ & 189.79 \\
\hline $\mathrm{tc} 2,3$ & 174.84 \\
$\mathrm{tc} 2,2$ & 202.34
\end{tabular}


Tabla A.2 Revisión de costos del diseño de red de la Figura A.2 desarrollado por Björk y Westerlund (2002) para el Caso de Estudio 5.1.

\begin{tabular}{|c|c|c|c|c|c|c|c|c|c|c|c|}
\hline $\begin{array}{l}\text { Número } \\
\text { de ID del } \\
\text { equipo }\end{array}$ & $\begin{array}{l}\text { Corrientes } \\
\text { que } \\
\text { participan }\end{array}$ & $\begin{array}{l}\text { Carga } \\
\text { Térmica } \\
{[\mathrm{kW}]}\end{array}$ & $\frac{\mathrm{hi}}{\left[\mathrm{kWm}^{-2} \mathrm{C}^{-1}\right]}$ & $\begin{array}{c}\text { ho } \\
{\left[\mathrm{kWm}^{-2}{ }^{-1} \mathrm{C}^{-1}\right]}\end{array}$ & $\frac{\mathrm{U}}{\left[\mathrm{kWm}^{-2}{ }^{\circ} \mathrm{C}^{-1}\right]}$ & $\begin{array}{l}\text { dth } \\
{\left[{ }^{\circ} \mathrm{C}\right]}\end{array}$ & $\begin{array}{l}\mathrm{dtc} \\
{\left[{ }^{\circ} \mathrm{C}\right]}\end{array}$ & $\begin{array}{c}\text { DMLT } \\
{\left[{ }^{\circ} \mathrm{C}\right]}\end{array}$ & $\begin{array}{c}\mathrm{A} \\
{\left[\mathrm{m}^{2}\right]}\end{array}$ & $\begin{array}{l}\text { Costo de capital } \\
\quad\left[\$ \mathrm{año}^{-1}\right]\end{array}$ & 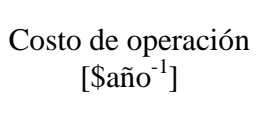 \\
\hline 1 & H1-C2 & 1139.28 & 0.15 & 0.10 & 0.06 & 3.04 & 22.02 & 9.58 & 1981.66 & 28024.01 & - \\
\hline 2 & $\mathrm{H} 2 \mathrm{a}-\mathrm{C} 2$ & 550.00 & 0.10 & 0.10 & 0.05 & 35.54 & 2.22 & 12.01 & 915.56 & 22022.15 & - \\
\hline 3 & $\mathrm{H} 2 \mathrm{~b}-\mathrm{C} 1$ & 1830.04 & 0.10 & 0.20 & 0.07 & 10.00 & 3.17 & 5.94 & 4617.45 & 40623.75 & - \\
\hline 4 & $\mathrm{H} 2-\mathrm{C} 1$ & 4819.96 & 0.10 & 0.20 & 0.07 & 2.79 & 20.00 & 8.73 & 8278.94 & 55879.05 & \\
\hline HU2 & HU-C2 & 1910.72 & 2.00 & 0.10 & 0.10 & 25.00 & 120.54 & 60.73 & 330.34 & 21213.34 & 110.00 \\
\hline CU1 & $\mathrm{H} 1-\mathrm{CU}$ & 2010.72 & 0.15 & 0.50 & 0.12 & 102.02 & 50.00 & 72.95 & 238.89 & 17397.08 & 10.00 \\
\hline & & & & & & \multicolumn{6}{|c|}{14381.18} \\
\hline & & \multicolumn{3}{|c|}{ Calor total recuperado $[\mathrm{kW}]$} & 8339.28 & \multicolumn{5}{|c|}{ Costo total anual de capital $\left[\$ a n ̃ o o^{-1}\right]$} & 185159.39 \\
\hline & & \multicolumn{3}{|c|}{$\begin{array}{l}\text { Total de servicios de } \\
\text { calentamiento }[\mathrm{kW}]\end{array}$} & 210178.69 & \multicolumn{5}{|c|}{ Costo total anual de operación $\left[\$ a n ̃ o^{-1}\right]$} & 230285.84 \\
\hline & & \multicolumn{3}{|c|}{$\begin{array}{l}\text { Total de servicios de } \\
\text { enfriamiento }[\mathrm{kW}]\end{array}$} & 20107.15 & \multicolumn{5}{|c|}{ Costo total anual de la red $\left[\$ a n ̃ o^{-1}\right]$} & $415,445.24$ \\
\hline & & \multicolumn{3}{|c|}{ Área total estimada $\left[\mathrm{m}^{2}\right]$} & 14381.18 & & & & & & \\
\hline
\end{tabular}

Cálculo de temperaturas intermedias calientes

\begin{tabular}{cc} 
th1,1 & 142.02 \\
\hline th2,1 & 179.18 \\
th 2,2 & 180.88 \\
th2,1-2 & 180.50 \\
\hline
\end{tabular}

Cálculo de temperaturas intermedias frías

tc $1,3 \quad 177.71$

tc2,4 $\quad 176.96$

tc2,1 204.46

\section{Cálculo de fracciones de} flujo

fh2,2 0.226093436 fh $2,3 \quad 0.773906564$ 


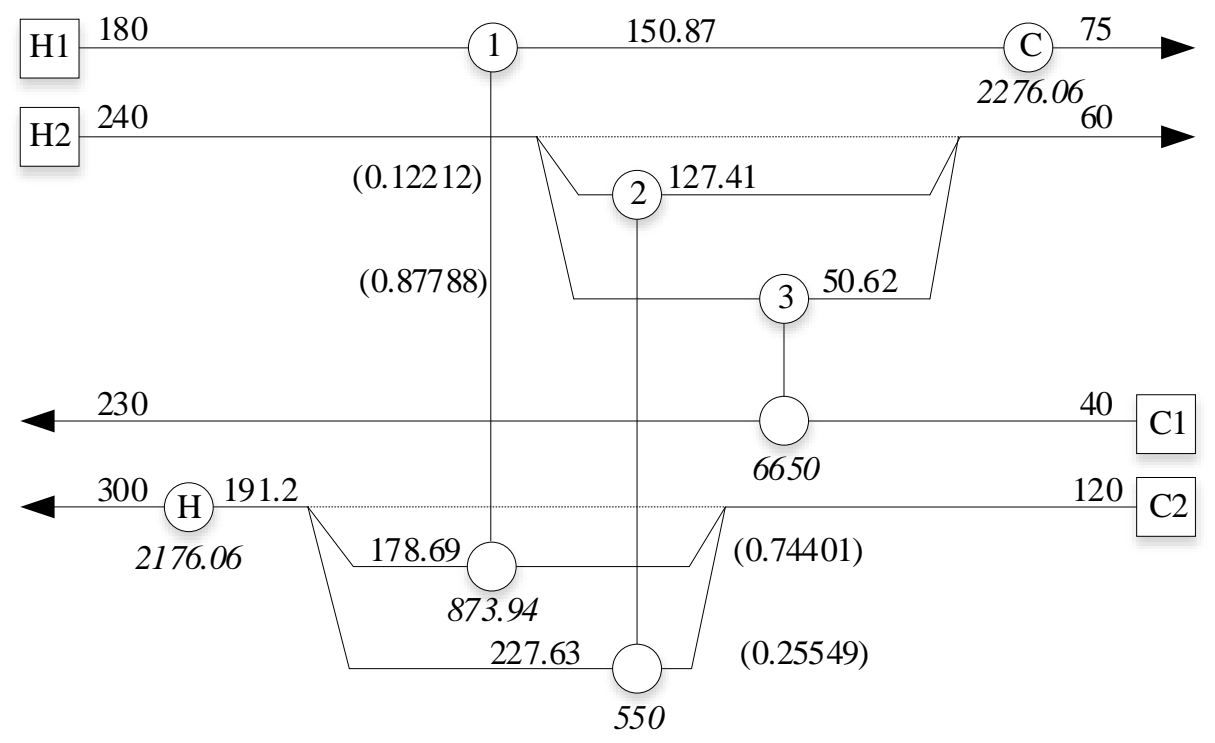

Figura A.3. Diseño de red determinado en este trabajo para el Caso de Estudio 5.1 con CTA de $\$ 411,624.48$. 
Tabla A.3 Revisión de costos del diseño de red de la Figura A.3 desarrollado en este trabajo para el Caso de Estudio 5.1.

\begin{tabular}{|c|c|c|c|c|c|c|c|c|c|c|c|}
\hline $\begin{array}{l}\text { Número } \\
\text { de ID del } \\
\text { equipo }\end{array}$ & $\begin{array}{l}\text { Corrientes } \\
\text { que } \\
\text { participan }\end{array}$ & $\begin{array}{c}\text { Carga } \\
\text { Térmica } \\
{[\mathrm{kW}]}\end{array}$ & $\begin{array}{c}\mathrm{hi} \\
{\left[\mathrm{kWm}^{-2 \circ} \mathrm{C}^{-1}\right]}\end{array}$ & $\begin{array}{c}\text { ho } \\
{\left[\mathrm{kWm}^{-2} \mathrm{C}^{-1}\right]}\end{array}$ & $\frac{\mathrm{U}}{\left[\mathrm{kWm}^{-2 \circ} \mathrm{C}^{-1}\right]}$ & $\begin{array}{l}\text { dth } \\
{\left[{ }^{\circ} \mathrm{C}\right]}\end{array}$ & $\begin{array}{l}\mathrm{dtc} \\
{\left[{ }^{\circ} \mathrm{C}\right]}\end{array}$ & $\begin{array}{c}\text { DMLT } \\
{\left[{ }^{\circ} \mathrm{C}\right]}\end{array}$ & $\begin{array}{c}\mathrm{A} \\
{\left[\mathrm{m}^{2}\right]}\end{array}$ & $\begin{array}{l}\text { Costo de capital } \\
\quad\left[\$ \mathrm{añono}^{-1}\right]\end{array}$ & $\begin{array}{c}\text { Costo de operación } \\
{\left[\$ \mathrm{añon}^{-1}\right]}\end{array}$ \\
\hline 1 & $\mathrm{H} 1-\mathrm{C} 2 \mathrm{a}$ & 873.94 & 0.15 & 0.10 & 0.06 & 1.31 & 30.87 & 9.35 & 1557.80 & 25743.10 & - \\
\hline 2 & $\mathrm{H} 2 \mathrm{a}-\mathrm{C} 2 \mathrm{~b}$ & 550.00 & 0.10 & 0.10 & 0.05 & 12.37 & 7.41 & 9.68 & 1136.67 & 23348.89 & - \\
\hline 3 & $\mathrm{H} 2 \mathrm{~b}-\mathrm{C} 1$ & 6650.00 & 0.10 & 0.20 & 0.07 & 10.00 & 10.62 & 10.31 & 9676.95 & 61313.96 & - \\
\hline HU2 & HU-C2 & 2176.06 & 2.00 & 0.10 & 0.10 & 25.00 & 133.80 & 64.86 & 352.27 & 21541.17 & 110.00 \\
\hline CU1 & $\mathrm{H} 1-\mathrm{CU}$ & 2276.06 & 0.15 & 0.50 & 0.12 & 110.87 & 50.00 & 76.44 & 258.07 & 17549.80 & 10.00 \\
\hline & & \multicolumn{3}{|c|}{ Calor total recuperado $[\mathrm{kW}]$} & 12526.06 & \multicolumn{5}{|c|}{ Costo total anual de capital $\left[\$\right.$ año $\left.^{-1}\right]$} & 149496.92 \\
\hline & & \multicolumn{3}{|c|}{$\begin{array}{l}\text { Total de servicios de } \\
\text { calentamiento }[\mathrm{kW}]\end{array}$} & 239366.93 & \multicolumn{5}{|c|}{ Costo total anual de operación [ $\$$ año $\left.{ }^{-1}\right]$} & 262127.56 \\
\hline & & \multicolumn{3}{|c|}{$\begin{array}{l}\text { Total de servicios de } \\
\text { enfriamiento }[\mathrm{kW}]\end{array}$} & 22760.63 & \multicolumn{5}{|c|}{ 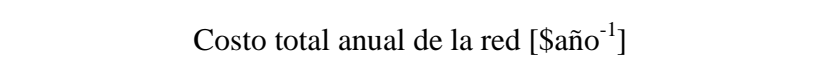 } & $411,624.48$ \\
\hline & & \multicolumn{3}{|c|}{ Área total estimada $\left[\mathrm{m}^{2}\right]$} & 12981.75 & & & & & & \\
\hline \multicolumn{2}{|c|}{$\begin{array}{l}\text { Cálculo de temperaturas } \\
\text { intermedias calientes }\end{array}$} & \multicolumn{3}{|c|}{$\begin{array}{c}\text { Cálculo de temperaturas } \\
\text { intermedias frías }\end{array}$} & & \multirow{2}{*}{\multicolumn{4}{|c|}{$\begin{array}{l}\text { Cálculo de fracciones de } \\
\text { flujo }\end{array}$}} & & \\
\hline th1,1 & 150.87 & & $\mathrm{tc} 2,1$ & 178.69 & & & & & & & \\
\hline th2,2 & 127.41 & & $\mathrm{tc} 2,3$ & 227.63 & & fh 2,2 & 0.122124868 & fh 2,3 & 0.877875132 & & \\
\hline th 2,3 & 50.62 & & $\mathrm{tc} 2,1-3$ & 191.20 & & $\mathrm{fc} 2,1$ & 0.744505699 & $\mathrm{fc} 2,3$ & 0.255494301 & & \\
\hline
\end{tabular}




\section{A.2. Revisión del Caso de Estudio 5.2}

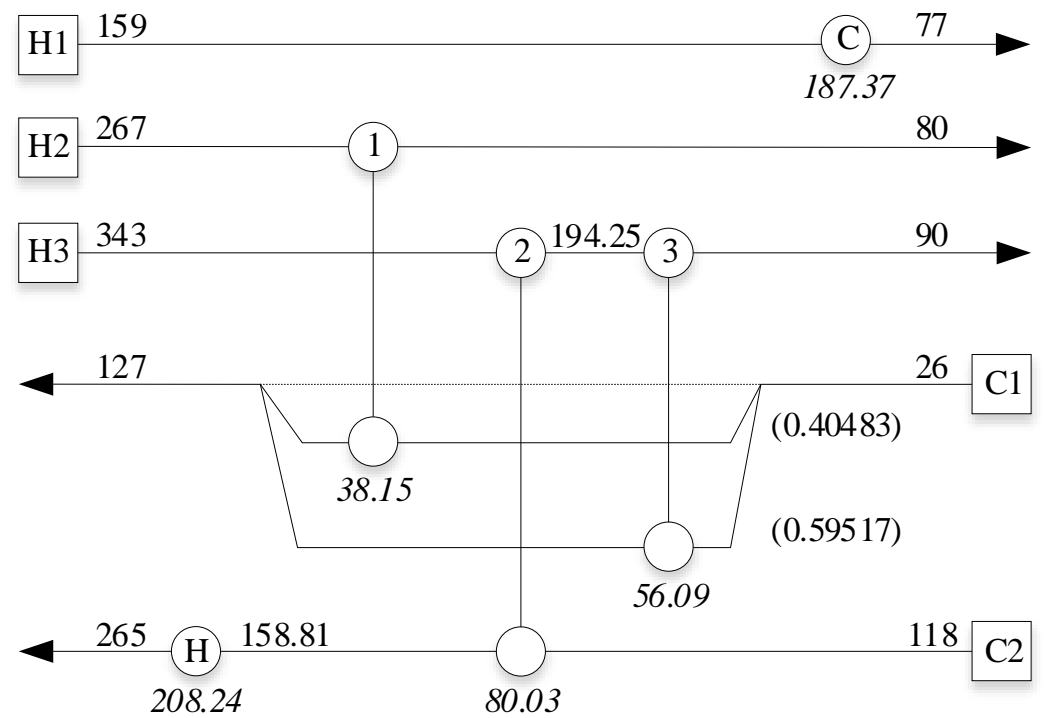

Figura A.4. Diseño de red determinado por Petterson (2008) para el Caso de Estudio 5.2 con $\mathrm{CTA}_{\mathrm{DMLT}}$ reportado de $\$ 80,962$ y $\mathrm{CTA}_{\mathrm{DMLT}}$ revisado de $\$ 80,959.55$.

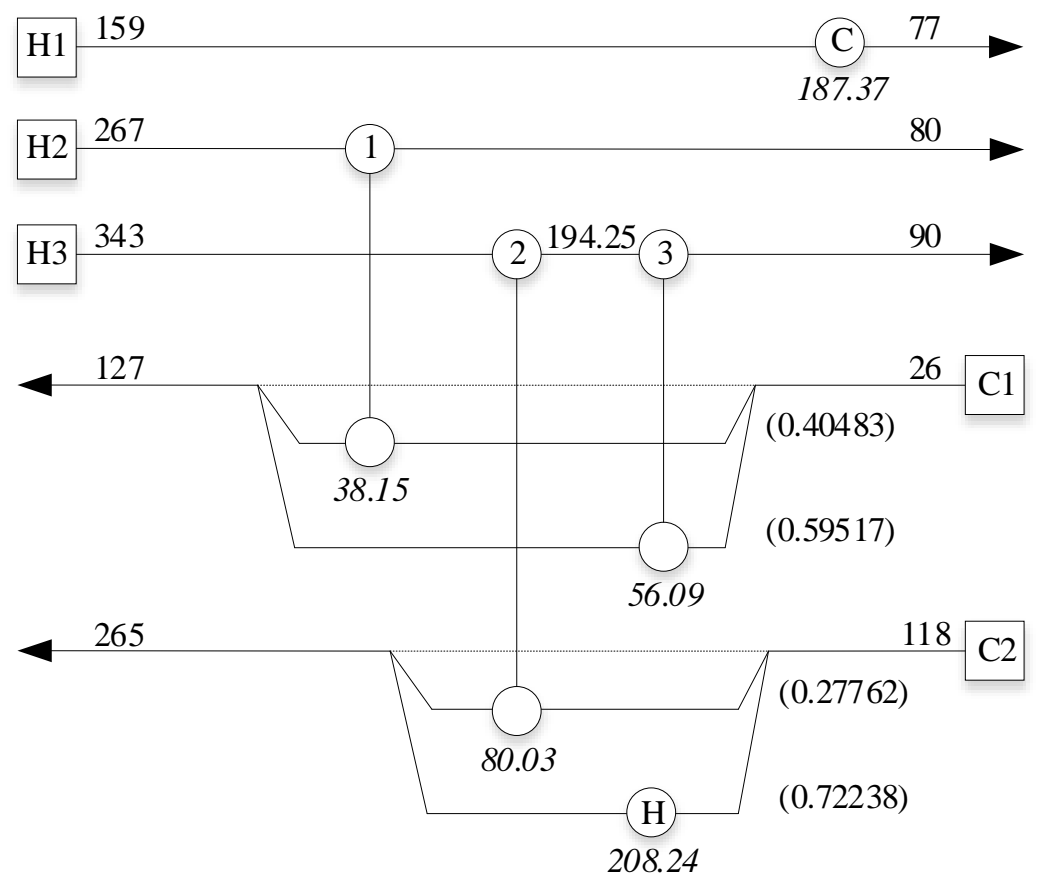

Figura A.5. Diseño de red determinado por Gupta y Ghosh (2010) para el Caso de Estudio 5.2 con $\mathrm{CTA}_{\text {Paterson }}$ reportado de $\$ 80,379$ y $\mathrm{CTA}_{\mathrm{DMLT}}$ revisado de $\$ 80,378.98$. 
Tabla A.4 Revisión de costos del diseño de red de la Figura A.4 desarrollado por Petterson (2008) para el Caso de Estudio 5.2.

\begin{tabular}{|c|c|c|c|c|c|c|c|c|c|c|c|}
\hline $\begin{array}{l}\text { Número } \\
\text { de ID del } \\
\text { equipo }\end{array}$ & $\begin{array}{l}\text { Corrientes } \\
\text { que } \\
\text { participan }\end{array}$ & $\begin{array}{c}\text { Carga } \\
\text { Térmica } \\
{[\mathrm{kW}]}\end{array}$ & $\begin{array}{c}\mathrm{hi} \\
{\left[\mathrm{kWm}^{-2}{ }^{\circ} \mathrm{C}^{-1}\right]}\end{array}$ & $\begin{array}{c}\text { ho } \\
{\left[\mathrm{kWm}^{-2}{ }^{\circ} \mathrm{C}^{-1}\right]}\end{array}$ & $\frac{\mathrm{U}}{\left[\mathrm{kWm}^{-2}{ }^{\circ} \mathrm{C}^{-1}\right]}$ & $\begin{array}{l}\text { dth } \\
{\left[{ }^{\circ} \mathrm{C}\right]}\end{array}$ & $\begin{array}{l}\mathrm{dtc} \\
{\left[{ }^{\circ} \mathrm{C}\right]}\end{array}$ & $\begin{array}{c}\text { DMLT } \\
{\left[{ }^{\circ} \mathrm{C}\right]}\end{array}$ & $\begin{array}{c}\mathrm{A} \\
{\left[\mathrm{m}^{2}\right]}\end{array}$ & $\begin{array}{l}\text { Costo de capital } \\
\quad\left[\$ \text { año }^{-1}\right]\end{array}$ & $\begin{array}{c}\text { Costo de operación } \\
{\left[\$ \text { año }^{-1}\right]}\end{array}$ \\
\hline 1 & $\mathrm{H} 2-\mathrm{C} 1 \mathrm{~b}$ & 38.15 & 0.04 & 0.01 & 0.01 & 140.00 & 54.00 & 90.27 & 52.82 & 11625.82 & - \\
\hline 3 & $\mathrm{H} 3-\mathrm{C} 1 \mathrm{a}$ & 56.09 & 0.50 & 0.01 & 0.01 & 67.25 & 64.00 & 65.61 & 87.19 & 14375.34 & - \\
\hline HU2 & HU-C2 & 208.24 & 0.05 & 0.50 & 0.05 & 35.00 & 141.19 & 76.13 & 60.17 & 12213.81 & 22906.18 \\
\hline CU1 & $\mathrm{H} 1-\mathrm{CU}$ & 187.37 & 0.10 & 0.20 & 0.07 & 99.00 & 57.00 & 76.08 & 36.94 & 10355.46 & 1873.70 \\
\hline \multicolumn{12}{|c|}{239.75} \\
\hline & & \multicolumn{3}{|c|}{ Calor total recuperado $[\mathrm{kW}]$} & 174.26 & \multicolumn{5}{|c|}{ Costo total anual de capital $\left[\$ a \tilde{n} o^{-1}\right]$} & 56179.67 \\
\hline & & \multicolumn{3}{|c|}{$\begin{array}{l}\text { Total de servicios de } \\
\text { calentamiento }[\mathrm{kW}]\end{array}$} & 208.24 & \multicolumn{5}{|c|}{ Costo total anual de operación [ $\left[\right.$ año $\left.0^{-1}\right]$} & 24779.88 \\
\hline & & \multicolumn{3}{|c|}{$\begin{array}{l}\text { Total de servicios de } \\
\text { enfriamiento }[\mathrm{kW}]\end{array}$} & 187.37 & \multicolumn{5}{|c|}{ Costo total anual de la red $\left[\$ a \tilde{a n o}^{-1}\right]$} & $80,959.55$ \\
\hline & & \multicolumn{3}{|c|}{ Área total estimada $\left[\mathrm{m}^{2}\right]$} & 239.75 & & & & & & \\
\hline
\end{tabular}

Cálculo de temperaturas intermedias calientes

th3,2 194.25

\section{Cálculo de temperaturas} intermedias frías

\begin{tabular}{ll}
$\mathrm{tc} 1,1$ & 127.00 \\
$\mathrm{tc} 1,3$ & 127.00 \\
\hline $\mathrm{tc} 2,2$ & 158.81
\end{tabular}

Cálculo de fracciones de

flujo

$\mathrm{fc} 1,1 \quad 0.404826335 \quad \mathrm{fc} 1,3 \quad 0.595173665$ 
Tabla A.5 Revisión de costos del diseño de red de la Figura A.5 desarrollado por Gupta y Ghosh (2010) para el Caso de Estudio 5.2.

\begin{tabular}{|c|c|c|c|c|c|c|c|c|c|c|c|}
\hline $\begin{array}{l}\text { Número } \\
\text { de ID del } \\
\text { equipo }\end{array}$ & $\begin{array}{l}\text { Corrientes } \\
\text { que } \\
\text { participan }\end{array}$ & $\begin{array}{c}\text { Carga } \\
\text { Térmica } \\
{[\mathrm{kW}]}\end{array}$ & $\begin{array}{c}\mathrm{hi} \\
{\left[\mathrm{kWm}^{-2} \mathrm{C}^{-1}\right]}\end{array}$ & $\begin{array}{c}\text { ho } \\
{\left[\mathrm{kWm}^{-2 \circ} \mathrm{C}^{-1}\right]}\end{array}$ & $\underset{\left[\mathrm{kWm}^{-2 \circ} \mathrm{C}^{-1}\right]}{\mathrm{U}}$ & $\begin{array}{l}\text { dth } \\
{\left[{ }^{\circ} \mathrm{C}\right]}\end{array}$ & $\begin{array}{l}\mathrm{dtc} \\
{\left[{ }^{\circ} \mathrm{C}\right]}\end{array}$ & $\begin{array}{c}\text { DMLT } \\
{\left[{ }^{\circ} \mathrm{C}\right]}\end{array}$ & $\begin{array}{c}\mathrm{A} \\
{\left[\mathrm{m}^{2}\right]}\end{array}$ & $\begin{array}{c}\text { Costo de capital } \\
{\left[\$ \mathrm{añno}^{-1}\right]}\end{array}$ & $\begin{array}{c}\text { Costo de operación } \\
{\left[\$ \text { \$año }^{-1}\right]}\end{array}$ \\
\hline 1 & $\mathrm{H} 2-\mathrm{C} 1 \mathrm{~b}$ & 38.15 & 0.04 & 0.01 & 0.01 & 140.00 & 54.00 & 90.27 & 52.82 & 11625.82 & - \\
\hline 2 & H3-C2 & 80.03 & 0.50 & 0.50 & 0.25 & 78.00 & 76.25 & 77.12 & 4.15 & 7732.07 & - \\
\hline 3 & $\mathrm{H} 3-\mathrm{C} 1 \mathrm{a}$ & 56.09 & 0.50 & 0.01 & 0.01 & 67.25 & 64.00 & 65.61 & 87.19 & 14375.34 & - \\
\hline HU2 & HU-C2 & 208.24 & 0.05 & 0.50 & 0.05 & 35.00 & 182.00 & 89.16 & 51.38 & 11510.42 & 22906.18 \\
\hline CU1 & $\mathrm{H} 1-\mathrm{CU}$ & 187.37 & 0.10 & 0.20 & 0.07 & 99.00 & 57.00 & 76.08 & 36.94 & 10355.46 & 1873.70 \\
\hline & & \multicolumn{3}{|c|}{ Calor total recuperado $[\mathrm{kW}]$} & 174.26 & \multicolumn{5}{|c|}{ Costo total anual de capital $\left[\$\right.$ año $\left.^{-1}\right]$} & 55599.10 \\
\hline & & \multicolumn{3}{|c|}{$\begin{array}{l}\text { Total de servicios de } \\
\text { calentamiento }[\mathrm{kW}]\end{array}$} & 208.24 & \multicolumn{5}{|c|}{ Costo total anual de operación $\left[\$ a n ̃ o^{-1}\right]$} & 24779.88 \\
\hline & & \multicolumn{3}{|c|}{$\begin{array}{l}\text { Total de servicios de } \\
\text { enfriamiento }[\mathrm{kW}]\end{array}$} & 187.37 & \multicolumn{5}{|c|}{ Costo total anual de la red $\left[\$ a n ̃ o o^{-1}\right]$} & $80,378.98$ \\
\hline & & \multicolumn{3}{|c|}{ Área total estimada $\left[\mathrm{m}^{2}\right]$} & 232.49 & & & & & & \\
\hline
\end{tabular}

Cálculo de temperaturas

intermedias calientes

th 3,2

194.25
Cálculo de temperaturas

intermedias frías

\begin{tabular}{cc}
$\mathrm{tc} 1,1$ & 127.00 \\
$\mathrm{tc} 1,3$ & 127.00 \\
\hline $\mathrm{tc} 2,2$ & 265.00 \\
$\mathrm{tc} 2, \mathrm{HU} 2$ & 265.00
\end{tabular}

Cálculo de fracciones de

flujo

$\mathrm{fc} 1,1 \quad 0.404826335 \quad \mathrm{fc} 1,3 \quad 0.595173665$

fc2,2 0.277621094 fc2,HU2 0.722378906 


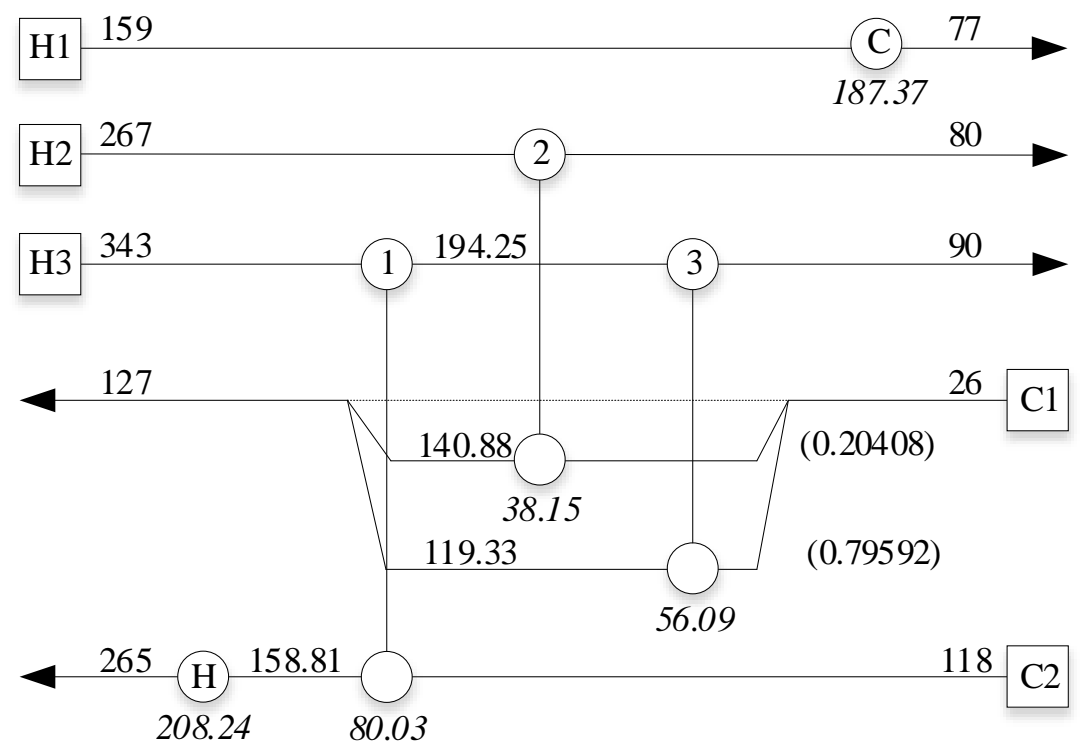

Figura A.6. Diseño de red determinado en este trabajo para el Caso de Estudio 5.2 con CTA de $\$ 80,847.71$. 
Tabla A.6 Revisión de costos del diseño de red de la Figura A.6 desarrollado en este trabajo para el Caso de Estudio 5.2.

\begin{tabular}{|c|c|c|c|c|c|c|c|c|c|c|c|}
\hline $\begin{array}{l}\text { Número } \\
\text { de ID del } \\
\text { equipo }\end{array}$ & $\begin{array}{l}\text { Corrientes } \\
\text { que } \\
\text { participan }\end{array}$ & $\begin{array}{c}\text { Carga } \\
\text { Térmica } \\
{[\mathrm{kW}]}\end{array}$ & $\underset{\left[\mathrm{kWm}^{-2 \circ} \mathrm{C}^{-1}\right]}{\mathrm{hi}}$ & $\begin{array}{c}\text { ho } \\
{\left[\mathrm{kWm}^{-2 \circ} \mathrm{C}^{-1}\right]}\end{array}$ & $\frac{\mathrm{U}}{\left[\mathrm{kWm}^{-2 \circ} \mathrm{C}^{-1}\right]}$ & $\begin{array}{l}\mathrm{dth} \\
{\left[{ }^{\circ} \mathrm{C}\right]}\end{array}$ & $\begin{array}{c}\mathrm{dtc} \\
{\left[{ }^{\circ} \mathrm{C}\right]}\end{array}$ & $\begin{array}{c}\text { DMLT } \\
{\left[{ }^{\circ} \mathrm{C}\right]}\end{array}$ & $\begin{array}{c}\mathrm{A} \\
{\left[\mathrm{m}^{2}\right]}\end{array}$ & $\begin{array}{c}\text { Costo de capital } \\
{\left[\$ \text { año }^{-1}\right]}\end{array}$ & $\begin{array}{c}\text { Costo de operación } \\
{\left[\$ \text { \año }^{-1}\right]}\end{array}$ \\
\hline HU2 & HU-C2 & 208.24 & 0.05 & 0.50 & 0.05 & 35.00 & 141.19 & 76.13 & 60.17 & 12213.81 & 22906.18 \\
\hline 1 & $\mathrm{H} 3-\mathrm{C} 2$ & 80.03 & 0.50 & 0.50 & 0.25 & 184.19 & 76.25 & 122.39 & 2.62 & 7609.25 & - \\
\hline 2 & $\mathrm{H} 3-\mathrm{C} 1 \mathrm{a}$ & 56.09 & 0.50 & 0.01 & 0.01 & 74.92 & 64.00 & 69.32 & 82.53 & 14002.43 & - \\
\hline 3 & $\mathrm{H} 2-\mathrm{C} 1 \mathrm{~b}$ & 38.15 & 0.04 & 0.01 & 0.01 & 126.12 & 54.00 & 85.02 & 56.09 & 11886.89 & - \\
\hline \multirow[t]{5}{*}{ CU1 } & $\mathrm{H} 1-\mathrm{CU}$ & 187.37 & 0.10 & 0.20 & 0.07 & 99.00 & 57.00 & 76.08 & 36.94 & 10355.46 & 1873.70 \\
\hline & & \multicolumn{3}{|c|}{ Calor total recuperado $[\mathrm{kW}]$} & 174.26 & \multicolumn{5}{|c|}{ Costo total anual de capital $\left[\$ a \tilde{n} o^{-1}\right]$} & 56067.83 \\
\hline & & \multicolumn{3}{|c|}{$\begin{array}{l}\text { Total de servicios de } \\
\text { calentamiento }[\mathrm{kW}]\end{array}$} & 208.24 & \multicolumn{5}{|c|}{ Costo total anual de operación [ $\$$ año $\left.{ }^{-1}\right]$} & 24779.88 \\
\hline & & \multicolumn{3}{|c|}{$\begin{array}{l}\text { Total de servicios de } \\
\text { enfriamiento }[\mathrm{kW}]\end{array}$} & 187.37 & \multicolumn{5}{|c|}{ Costo total anual de la red $\left[\$ a n ̃ o^{-1}\right]$} & $80,847.71$ \\
\hline & & \multicolumn{3}{|c|}{ Área total estimada $\left[\mathrm{m}^{2}\right]$} & 238.35 & & & & & & \\
\hline
\end{tabular}

Cálculo de temperaturas

intermedias calientes

th 3,1

194.25
Cálculo de temperaturas

\begin{tabular}{ll}
$\mathrm{tc} 1,2$ & 119.33 \\
$\mathrm{tc} 1,3$ & 140.88 \\
\hline $\mathrm{tc} 2,1$ & 158.81
\end{tabular}

Cálculo de fracciones de

flujo

fc1,2 $0.644094756 \quad f c 1,3 \quad 0.355905244$ 


\section{A.3. Revisión del Caso de Estudio 5.3}

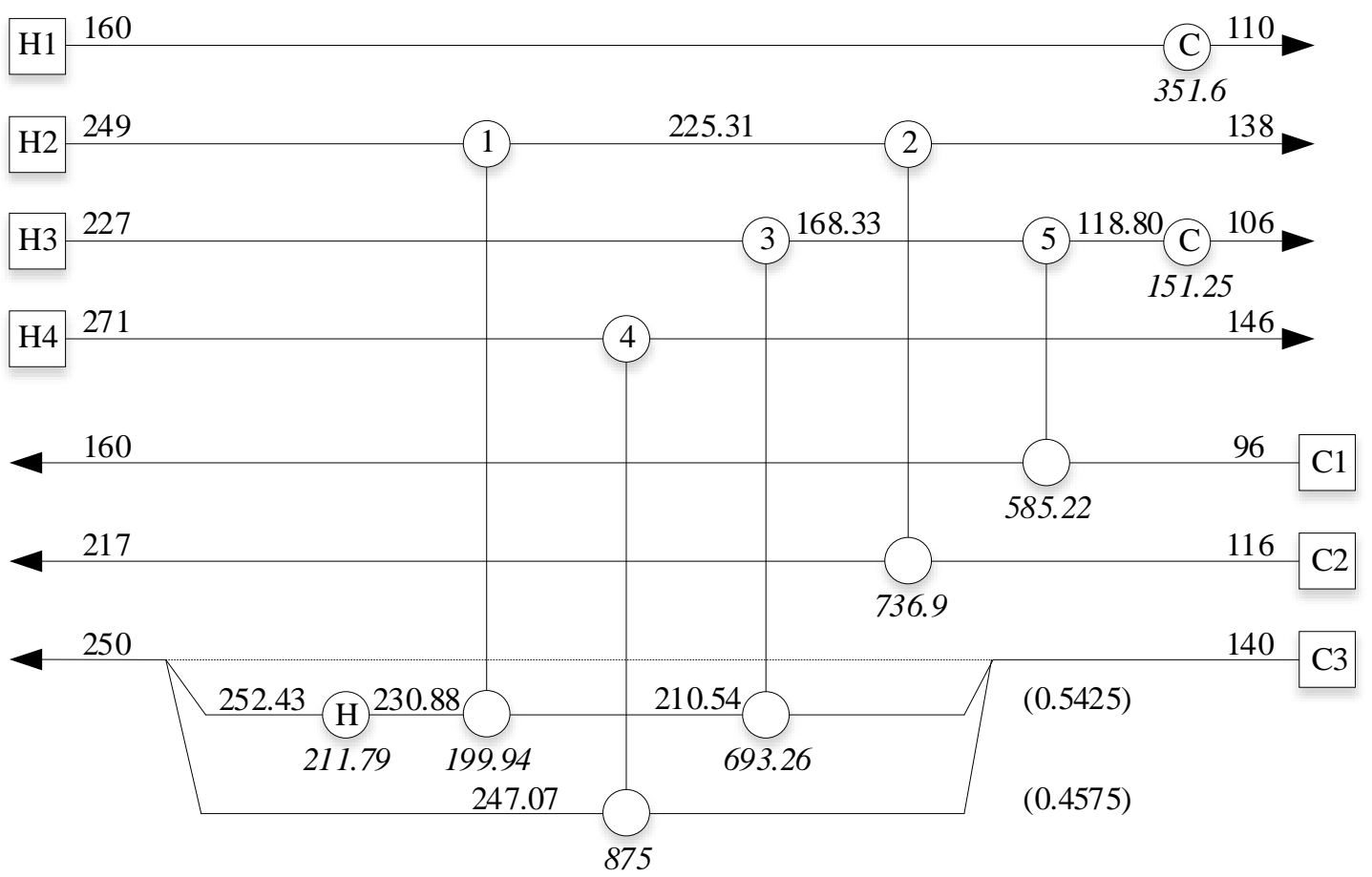

Figura A.7. Diseño de red determinado por Huang y Karimi (2013) con CTAPaterson reportado de $\$ 105,403$ y CTA $A_{\text {DMLT }}$ revisado de $\$ 105,421.83$. 
Tabla A.7 Revisión de costos del diseño de red de la Figura A.7 desarrollado por Luo y col. (2004) para el Caso de Estudio 5.3.

\begin{tabular}{|c|c|c|c|c|c|c|c|c|c|c|c|}
\hline $\begin{array}{l}\text { Número } \\
\text { de ID del } \\
\text { equipo }\end{array}$ & $\begin{array}{l}\text { Corrientes } \\
\text { que } \\
\text { participan }\end{array}$ & $\begin{array}{c}\text { Carga } \\
\text { Térmica } \\
{[\mathrm{kW}]}\end{array}$ & $\begin{array}{c}\mathrm{hi} \\
{\left[\mathrm{kWm}^{-2 \circ} \mathrm{C}^{-1}\right]}\end{array}$ & $\begin{array}{c}\text { ho } \\
{\left[\mathrm{kWm}^{-2}{ }^{\circ} \mathrm{C}^{-1}\right]}\end{array}$ & $\frac{\mathrm{U}}{\left[\mathrm{kWm}^{-2 \circ} \mathrm{C}^{-1}\right]}$ & $\begin{array}{l}\mathrm{dth} \\
{\left[{ }^{\circ} \mathrm{C}\right]}\end{array}$ & $\begin{array}{l}\mathrm{dtc} \\
{\left[{ }^{\circ} \mathrm{C}\right]}\end{array}$ & $\begin{array}{c}\text { DMLT } \\
{\left[{ }^{\circ} \mathrm{C}\right]}\end{array}$ & $\begin{array}{c}\mathrm{A} \\
{\left[\mathrm{m}^{2}\right]}\end{array}$ & $\begin{array}{c}\text { Costo de capital } \\
{\left[\$ \mathrm{Qaño}^{-1}\right]}\end{array}$ & $\begin{array}{c}\text { Costo de operación } \\
{\left[\$ \text { año }^{-1}\right]}\end{array}$ \\
\hline 1 & $\mathrm{H} 2-\mathrm{C} 3 \mathrm{a}$ & 199.94 & 1.60 & 1.60 & 0.80 & 18.12 & 14.77 & 16.39 & 15.25 & 6666.94 & - \\
\hline 2 & $\mathrm{H} 4-\mathrm{C} 3 \mathrm{~b}$ & 875.00 & 1.60 & 1.60 & 0.80 & 23.92 & 6.00 & 12.96 & 84.40 & 18609.87 & - \\
\hline 3 & $\mathrm{H} 3-\mathrm{C} 3 \mathrm{a}$ & 693.26 & 1.60 & 1.60 & 0.80 & 16.46 & 28.33 & 21.86 & 39.64 & 11825.81 & - \\
\hline 4 & $\mathrm{H} 2-\mathrm{C} 2$ & 736.90 & 1.60 & 1.60 & 0.80 & 8.31 & 22.00 & 14.06 & 65.51 & 15985.10 & - \\
\hline 5 & H3-C1 & 585.22 & 1.60 & 1.60 & 0.80 & 8.33 & 22.80 & 14.37 & 50.91 & 13740.58 & - \\
\hline CU1 & H1-CU & 351.60 & 1.60 & 1.60 & 0.80 & 70.00 & 40.00 & 53.61 & 8.20 & 4593.88 & 7032.00 \\
\hline CU3 & $\mathrm{H} 3-\mathrm{CU}$ & 151.26 & 1.60 & 1.60 & 0.80 & 28.80 & 36.00 & 32.27 & 5.86 & 3755.46 & 3025.11 \\
\hline \multicolumn{12}{|c|}{274.35} \\
\hline & & \multicolumn{3}{|c|}{ Calor total recuperado $[\mathrm{kW}]$} & 3090.32 & & \multicolumn{4}{|c|}{ Costo total anual de capital $\left[\$ \mathrm{año}^{-1}\right]$} & 78421.42 \\
\hline & & \multicolumn{3}{|c|}{$\begin{array}{l}\text { Total de servicios de } \\
\text { calentamiento }[\mathrm{kW}]\end{array}$} & 211.79 & & \multicolumn{4}{|c|}{ Costo total anual de operación $\left[\$\right.$ añ̃o $\left.^{-1}\right]$} & 27000.41 \\
\hline & & \multicolumn{3}{|c|}{$\begin{array}{l}\text { Total de servicios de } \\
\text { enfriamiento }[\mathrm{kW}]\end{array}$} & 502.86 & & \multicolumn{4}{|c|}{ Costo total anual de la red $\left[\$ \mathrm{año}^{-1}\right]$} & $105,421.83$ \\
\hline & & \multicolumn{3}{|c|}{ Área total estimada $\left[\mathrm{m}^{2}\right]$} & 274.35 & & & & & & \\
\hline
\end{tabular}

Cálculo de temperaturas intermedias calientes

\begin{tabular}{rr} 
th2,1 & 225.31 \\
\hline th 3,3 & 168.33 \\
th 3,5 & 118.80
\end{tabular}

Cálculo de temperaturas intermedias frías

$\begin{array}{cc}\text { tc3,HU3 } & 252.43 \\ \text { tc } 3,1 & 230.88 \\ \text { tc } 3,3 & 210.54 \\ \text { tc } 3,4 & 247.08\end{array}$

Cálculo de fracciones de

flujo 


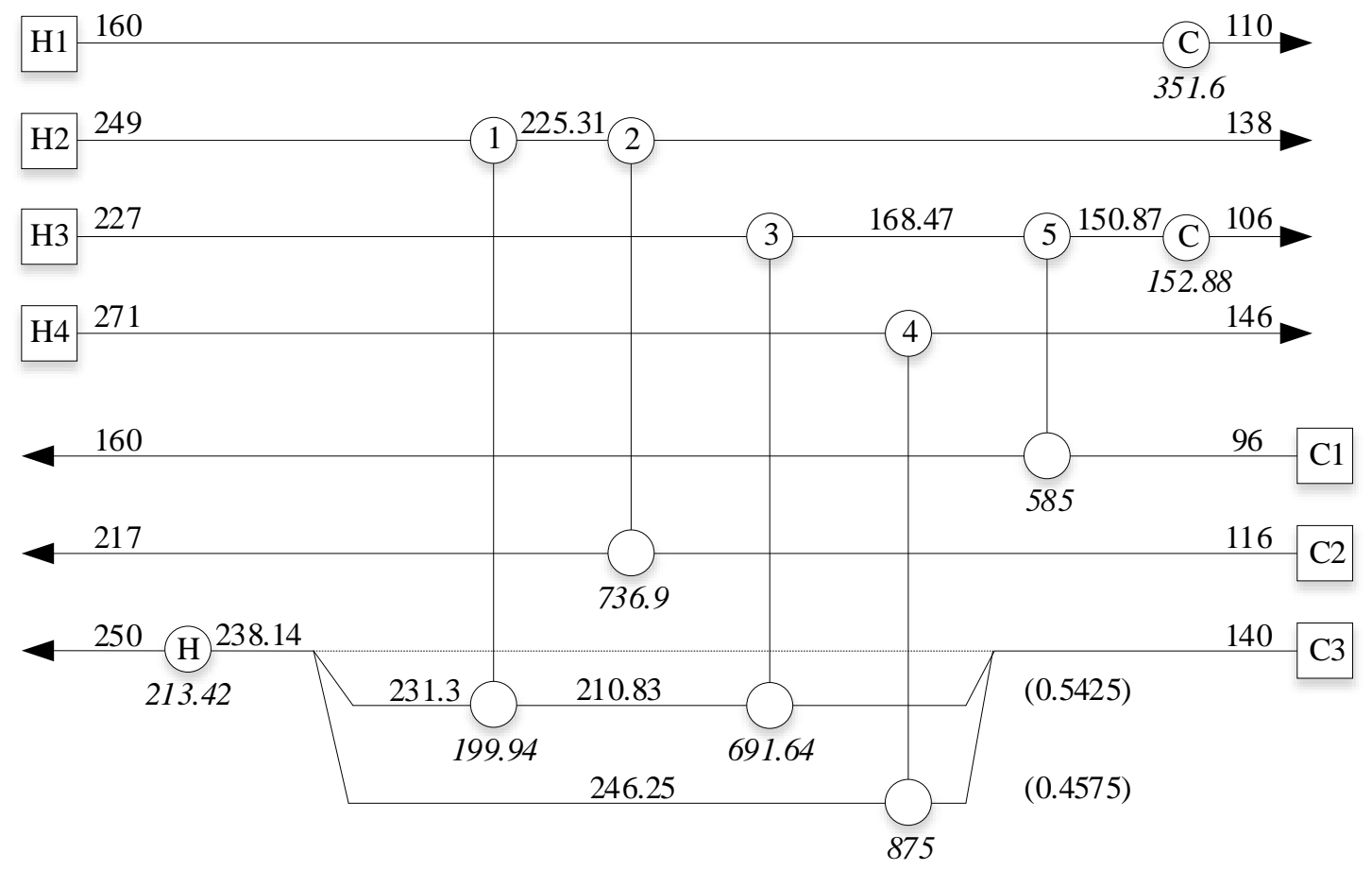

Figura A.8. Diseño de red determinado en este trabajo para el Caso de Estudio 5.3 con CTA de $\$ 105,481.12$. 
Tabla A.8 Revisión de costos del diseño de red de la Figura A.8 desarrollado en este trabajo para el Caso de Estudio 5.3.

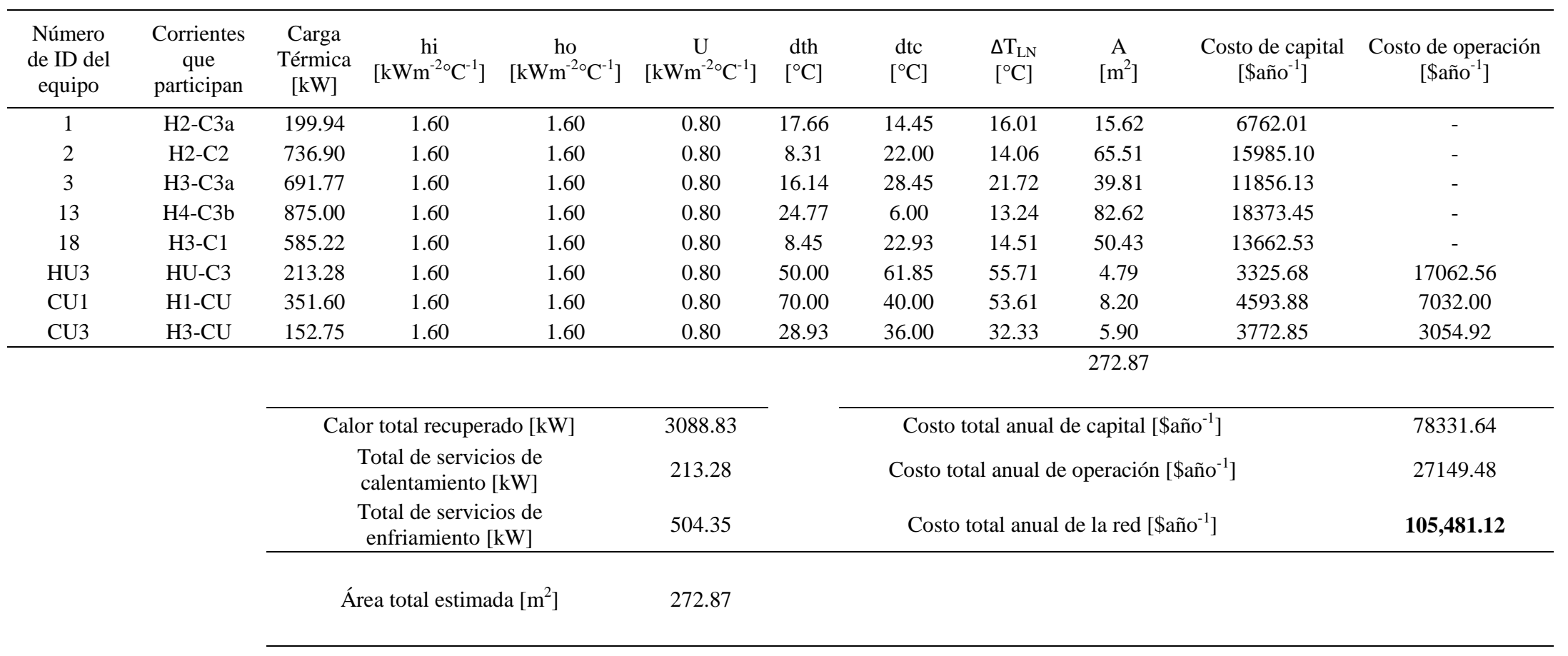

Cálculo de temperaturas intermedias calientes

\begin{tabular}{cc} 
th2,1 & 225.31 \\
\hline th3,3 & 168.45 \\
th 3,18 & 118.93
\end{tabular}

Cálculo de temperaturas intermedias frías

$\begin{array}{cc}\text { tc } 3,3 & 210.86 \\ \text { tc } 3,1 & 231.34 \\ \text { tc } 3,4 & 246.23 \\ \text { tc } 3,1-4 & 238.15\end{array}$

Cálculo de fracciones de

flujo

fc1,1 $0.542392554 \quad$ fc1,3 0.457607446 


\section{A.4. Revisión del Caso de Estudio 5.4}

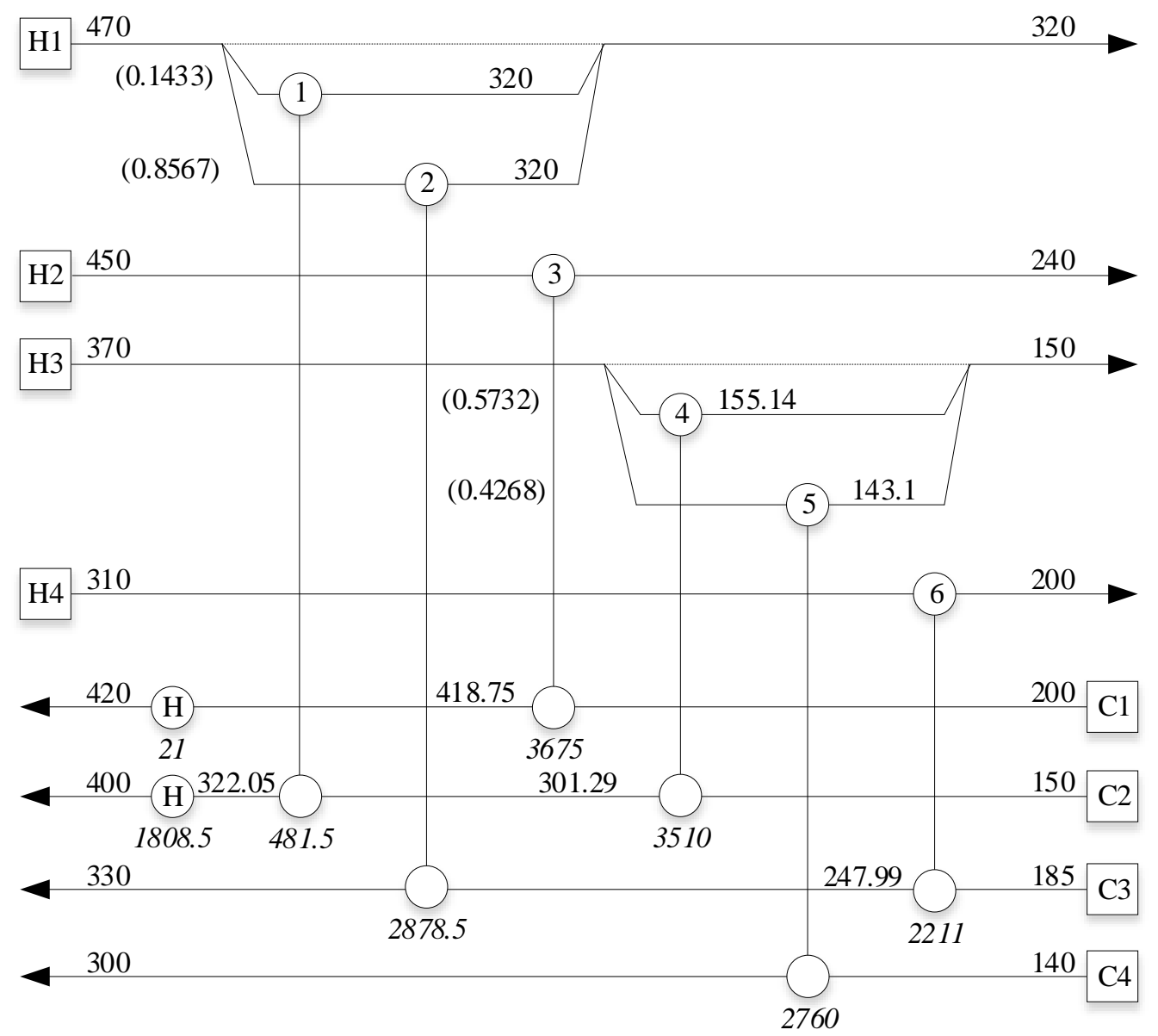

Figura A.9. Diseño de red determinado por Luo y col. (2004) para el Caso de Estudio 5.4 con

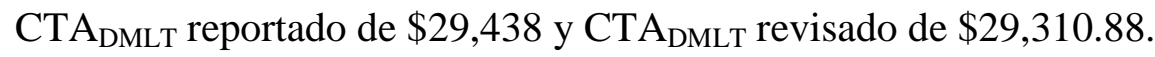


Tabla A.9 Revisión de costos del diseño de red de la Figura A.9 desarrollado por Luo y col. (2004) para el Caso de Estudio 5.4.

\begin{tabular}{|c|c|c|c|c|c|c|c|c|c|c|c|}
\hline $\begin{array}{c}\text { Número } \\
\text { de ID } \\
\text { del } \\
\text { equipo }\end{array}$ & $\begin{array}{l}\text { Corrientes } \\
\text { que } \\
\text { participan }\end{array}$ & $\begin{array}{c}\text { Carga } \\
\text { Térmica } \\
{\left[\mathrm{kBtu} \mathrm{h}^{-1}\right]}\end{array}$ & $\frac{\mathrm{hi}}{\left[\mathrm{kBtu}\left(\mathrm{h} \mathrm{ft}^{2}{ }^{\circ} \mathrm{F}\right)^{-1}\right]}$ & $\begin{array}{c}\text { ho } \\
{\left[\mathrm{kBtu}\left(\mathrm{h} \mathrm{ft}^{2}{ }^{\circ} \mathrm{F}\right)^{-1}\right]}\end{array}$ & 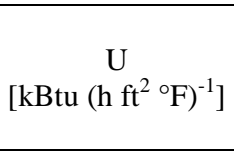 & $\begin{array}{l}\text { dth } \\
{\left[{ }^{\circ} \mathrm{F}\right]}\end{array}$ & $\begin{array}{c}\mathrm{dtc} \\
{\left[{ }^{\circ} \mathrm{F}\right]}\end{array}$ & $\begin{array}{c}\text { DMLT } \\
{\left[{ }^{\circ} \mathrm{F}\right]}\end{array}$ & $\begin{array}{c}\mathrm{A} \\
{\left[\mathrm{ft}^{2}\right]}\end{array}$ & $\begin{array}{c}\text { Costo de } \\
\text { capital } \\
{\left[\$ a \tilde{n} o^{-1}\right]}\end{array}$ & $\begin{array}{c}\text { Costo de } \\
\text { operación } \\
{\left[\$ \text { año }^{-1}\right]}\end{array}$ \\
\hline 1 & $\mathrm{H} 1 \mathrm{a}-\mathrm{C} 2$ & 481.50 & 0.30 & 0.30 & 0.15 & 147.95 & 18.71 & 62.50 & 51.36 & 371.92 & - \\
\hline 2 & $\mathrm{H} 1 \mathrm{~b}-\mathrm{C} 3$ & 2878.50 & 0.30 & 0.30 & 0.15 & 140.00 & 72.01 & 102.26 & 187.65 & 809.24 & - \\
\hline 3 & $\mathrm{H} 2-\mathrm{C} 1$ & 3675.00 & 0.30 & 0.30 & 0.15 & 31.25 & 40.00 & 35.45 & 691.21 & 1769.43 & - \\
\hline 4 & $\mathrm{H} 3 \mathrm{a}-\mathrm{C} 2$ & 3510.00 & 0.30 & 0.30 & 0.15 & 68.71 & 5.14 & 24.52 & 954.45 & 2147.43 & - \\
\hline 5 & $\mathrm{H} 3 \mathrm{a}-\mathrm{C} 4$ & 2760.00 & 0.30 & 0.30 & 0.15 & 70.00 & 3.10 & 21.46 & 857.55 & 2013.84 & - \\
\hline HU1 & HU-C1 & 21.00 & 0.60 & 0.30 & 0.20 & 36.00 & 37.25 & 36.62 & 2.87 & 65.85 & 232.05 \\
\hline HU2 & HU-C2 & 1808.50 & 0.60 & 0.30 & 0.20 & 56.00 & 133.95 & 89.38 & 101.17 & 558.59 & 19983.93 \\
\hline
\end{tabular}

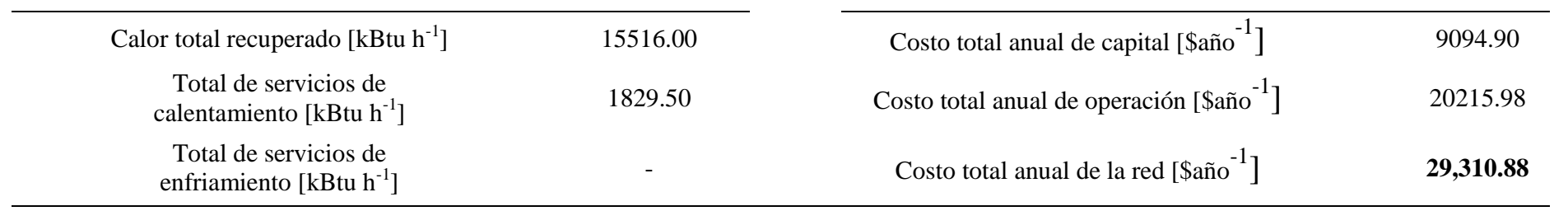

Área total estimada $\left[\mathrm{ft}^{2}\right]$

3291.27

Cálculo de

temperaturas

intermedias

calientes

\begin{tabular}{ll} 
th 1,1 & 320.00 \\
th1,2 & 320.00 \\
\hline th 3,4 & 155.14 \\
th 3,5 & 143.10
\end{tabular}

th3,5 143.10

\section{Cálculo de temperaturas}

intermedias frías

\begin{tabular}{ll}
$\mathrm{tc} 1,3$ & 418.75 \\
\hline $\mathrm{tc} 2,4$ & 301.29 \\
$\mathrm{tc} 2,1$ & 322.05 \\
\hline $\mathrm{tc} 3,6$ & 247.99
\end{tabular}

Cálculo de fracciones de

flujo

fh1,1 0.143303571 fh1,2 0.856696429

fh $3,4 \quad 0.573200664$ fh3,5 0.426799336 


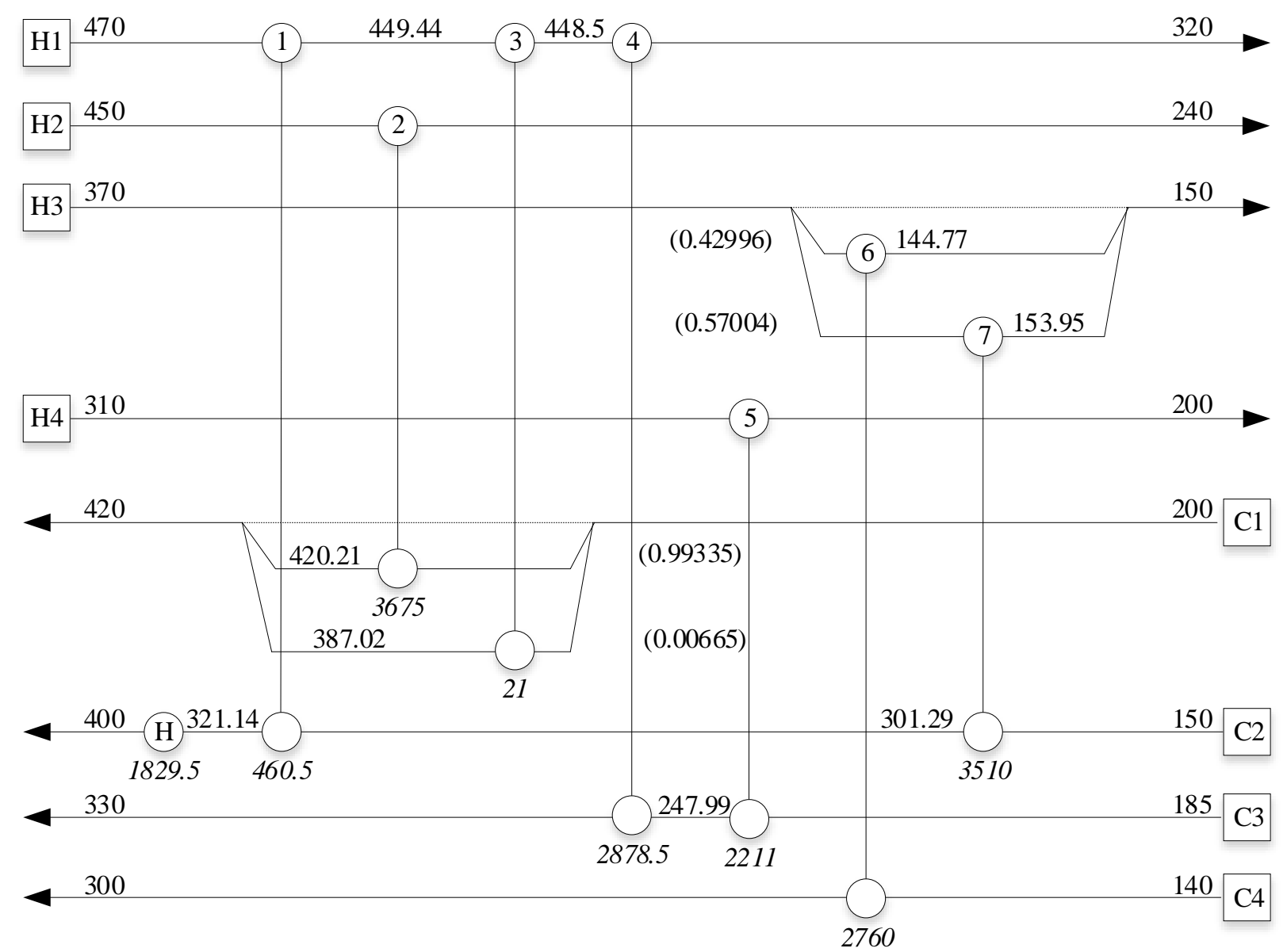

Figura A.10. Diseño de red determinado en este trabajo para el Caso de Estudio 5.4 con CTA $_{\text {DMLT }}$ de $\$ 29,156.65$. 
Tabla A.10 Revisión de costos del diseño de red de la Figura A.10 desarrollado en este trabajo para el Caso de Estudio 5.4.

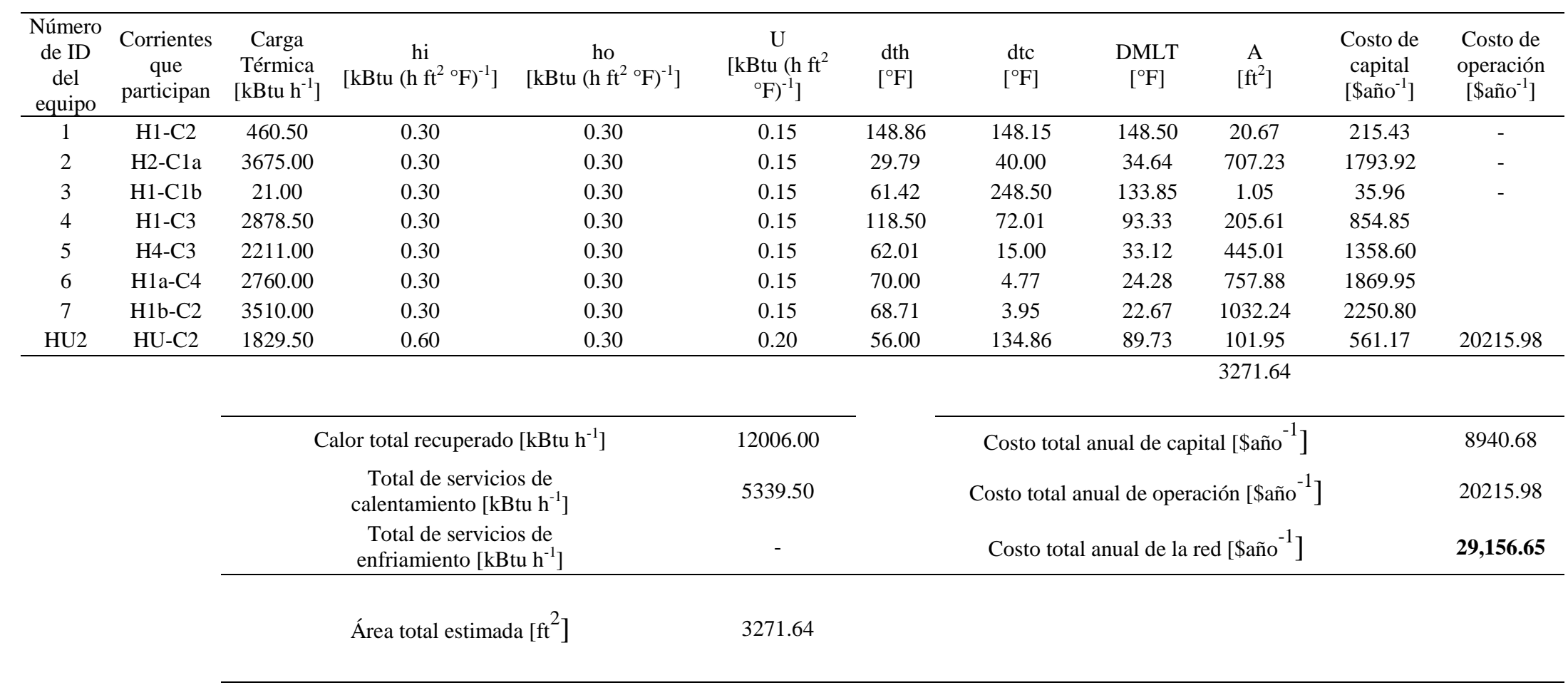

\begin{tabular}{cc}
\multicolumn{2}{c}{$\begin{array}{c}\text { Cálculo de } \\
\text { temperaturas } \\
\text { intermedias } \\
\text { calientes }\end{array}$} \\
\begin{tabular}{cc} 
th 1,1 & 449.44 \\
th 1,3 & 448.50 \\
\hline th 2,6 & 144.77 \\
th 3,7 & 153.95
\end{tabular}
\end{tabular}

\section{Cálculo de temperaturas} intermedias frías

\begin{tabular}{rl}
$\mathrm{tc} 1,2$ & 420.21 \\
$\mathrm{tc} 1,3$ & 388.02 \\
\hline $\mathrm{tc} 2,7$ & 301.29 \\
$\mathrm{tc} 2,1$ & 321.14 \\
\hline $\mathrm{tc} 3,5$ & 247.99
\end{tabular}

Cálculo de fracciones de flujo

\begin{tabular}{cccc} 
fh3,37 & 0.429962631 & fh3,39 & 0.570037369 \\
\hline fc 1,4 & 0.993351887 & fc1,17 & 0.006648113
\end{tabular}




\section{A.5. Revisión del Caso de Estudio 5.5}

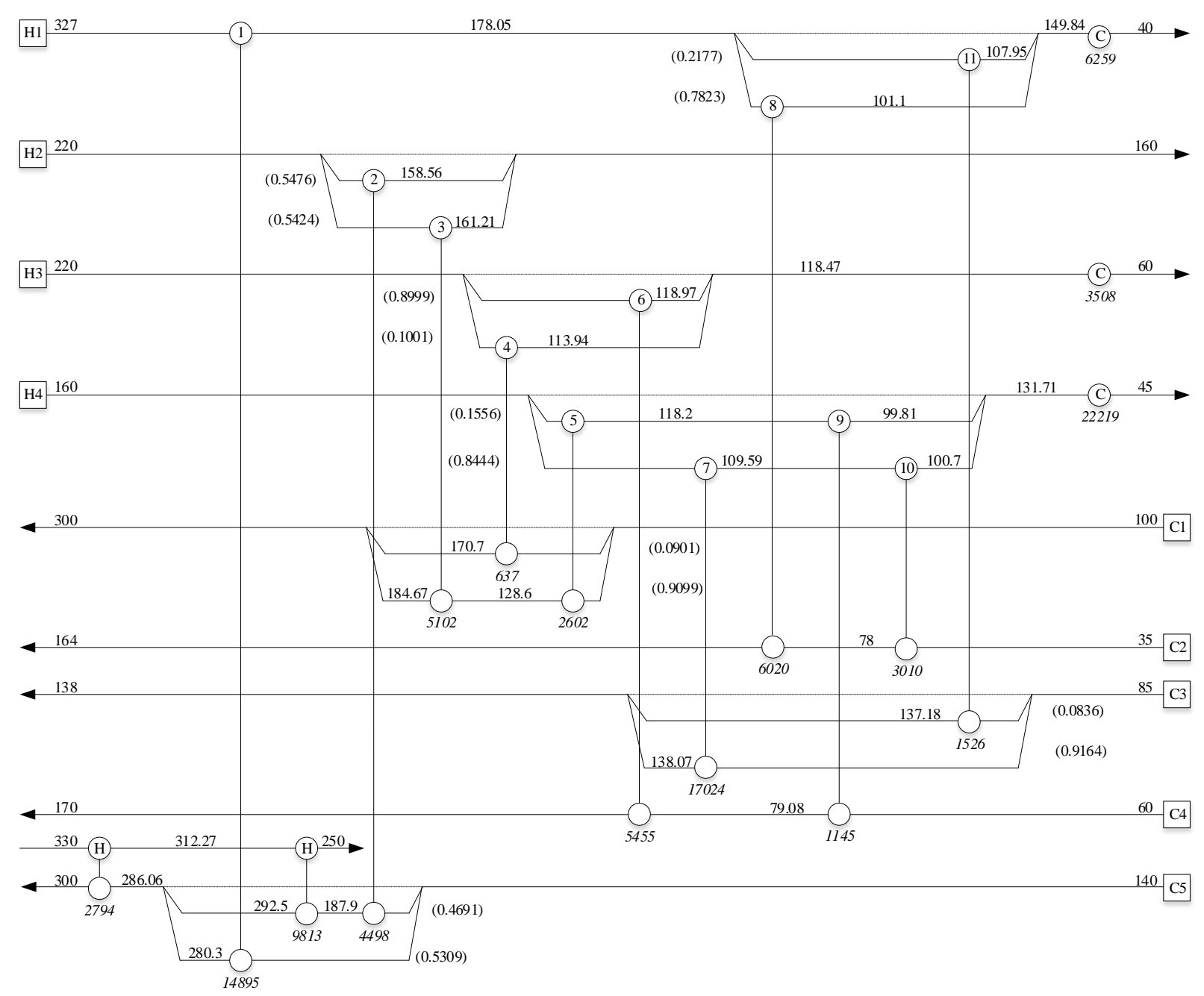

Figura A.11. Diseño de red determinado por Petterson (2005) para el Caso de Estudio 5.5 con $\mathrm{CTA}_{\text {DMLT }}$ reportado de $\$ 2,904,953$ y CTA $\mathrm{C}_{\text {DMLT }}$ revisado de $\$ 2,904,953.09$. 
Tabla A.11 Revisión de costos del diseño de red de la Figura A.11 desarrollado por Petterson (2005) para el Caso de Estudio 5.5.

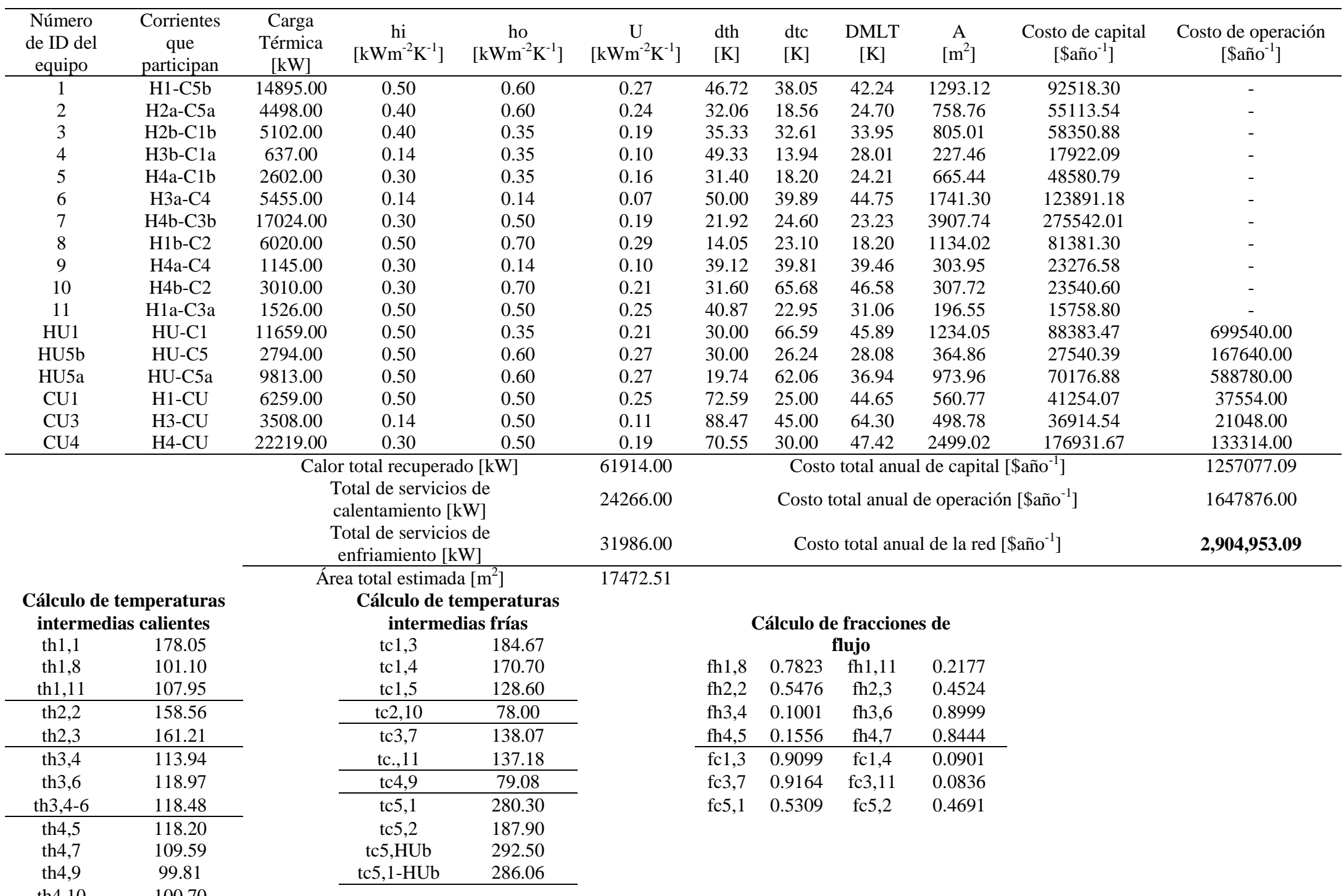




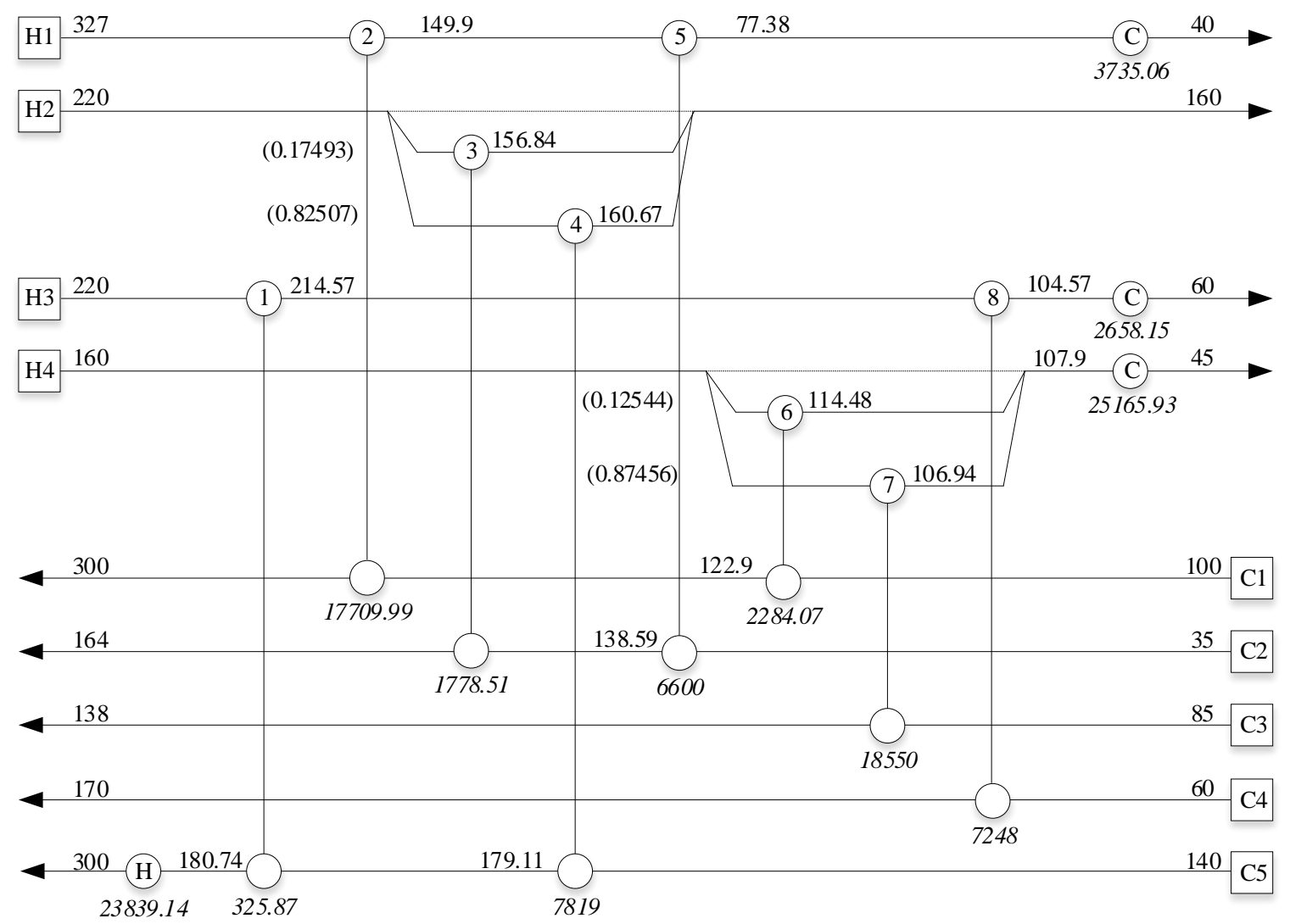

Figura A.12. Diseño de red determinado por Pavão y col. (2017b) para el Caso de Estudio 5.5 con $\mathrm{CTA}_{\mathrm{DMLT}}$ reportado de $\$ 2,919,675$ y CTA $\mathrm{C}_{\mathrm{DMLT}}$ revisado de $\$ 2,919,675.73$. 
Tabla A.12 Revisión de costos del diseño de red de la Figura A.12 desarrollado por Pavão y col. (2017b) para el Caso de Estudio 5.5 .

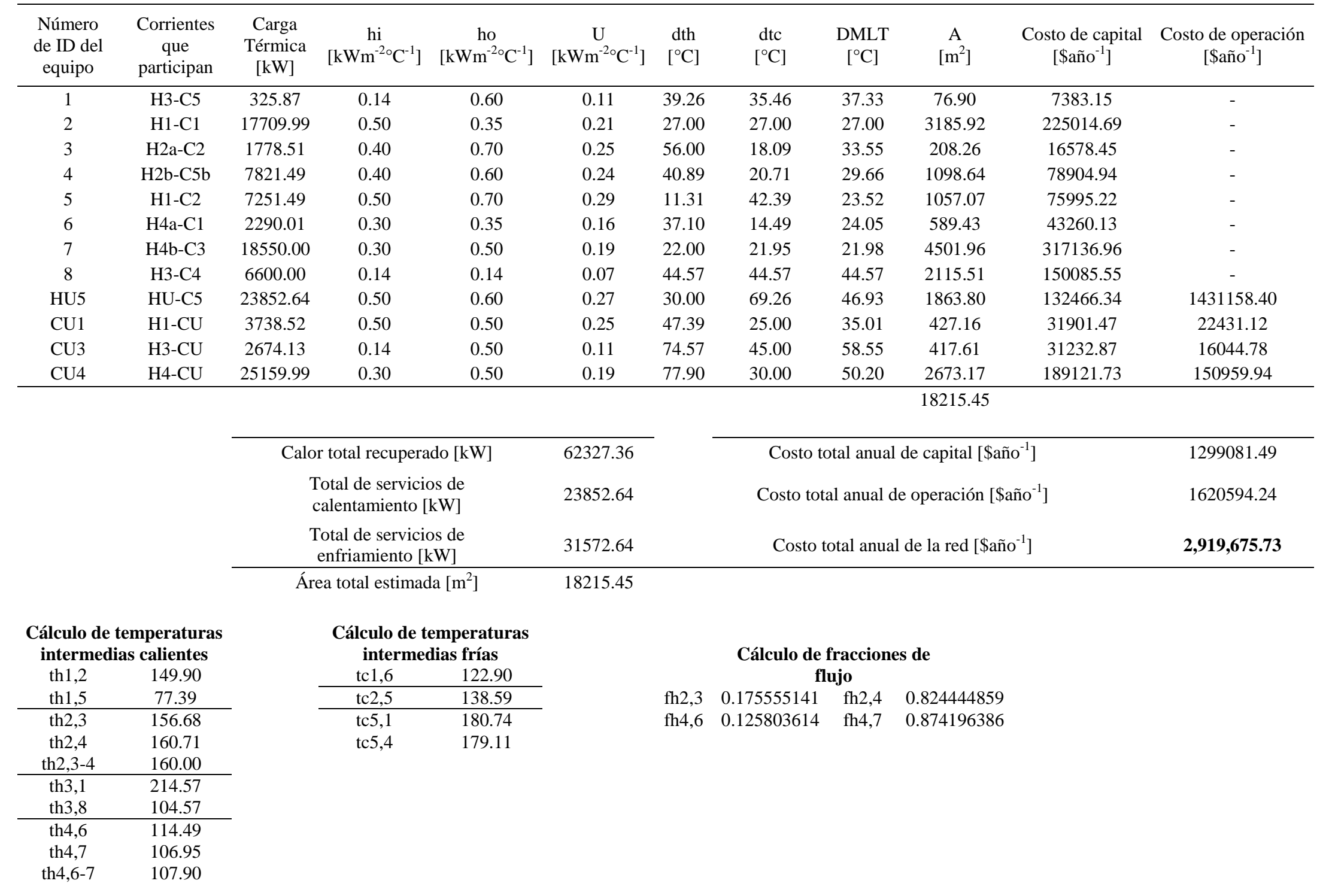




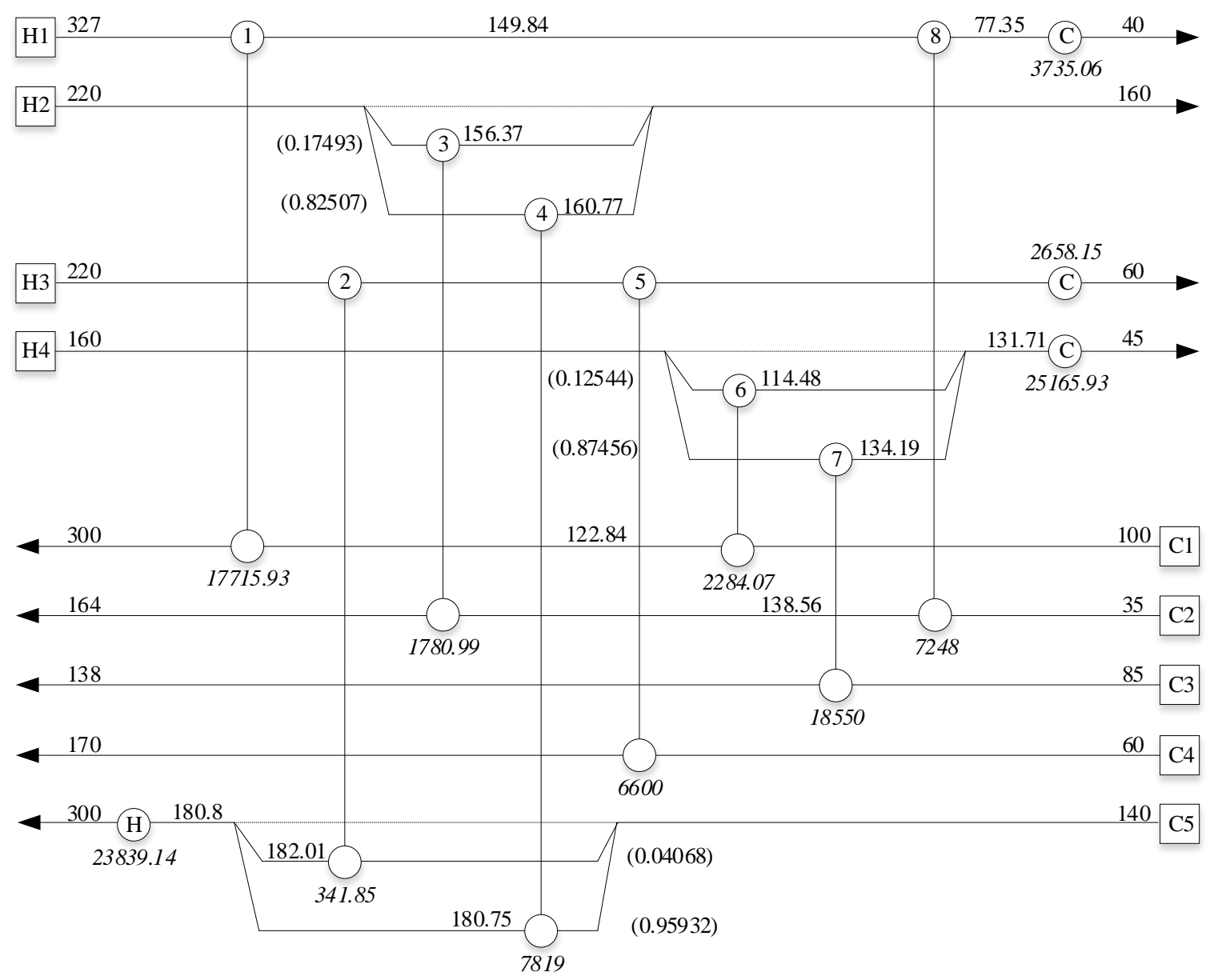

Figura A.13. Diseño de red determinado en este trabajo para el Caso de Estudio 5.5 con CTA $_{\text {DMLT }}$ de $\$ 2,919,687.66$. 
Tabla A.13 Revisión de costos del diseño de red de la Figura A.12 desarrollado en este trabajo para el Caso de Estudio 5.5.

\begin{tabular}{|c|c|c|c|c|c|c|c|c|c|c|c|}
\hline $\begin{array}{l}\text { Número } \\
\text { de ID del } \\
\text { equipo }\end{array}$ & $\begin{array}{l}\text { Corrientes } \\
\text { que } \\
\text { participan }\end{array}$ & $\begin{array}{c}\text { Carga } \\
\text { Térmica } \\
{[\mathrm{kW}]}\end{array}$ & $\begin{array}{c}\mathrm{hi} \\
{\left[\mathrm{kWm}^{-2} \mathrm{~K}^{-1}\right]}\end{array}$ & $\begin{array}{c}\text { ho } \\
{\left[\mathrm{kWm}^{-2} \mathrm{~K}^{-1}\right]}\end{array}$ & $\begin{array}{c}\mathrm{U} \\
{\left[\mathrm{kWm}^{-2} \mathrm{~K}^{-1}\right]}\end{array}$ & $\begin{array}{l}\text { dth } \\
{[\mathrm{K}]}\end{array}$ & $\begin{array}{l}\text { dtc } \\
{[\mathrm{K}]}\end{array}$ & $\begin{array}{c}\Delta \mathrm{T}_{\mathrm{LN}} \\
{[\mathrm{K}]}\end{array}$ & $\begin{array}{c}\mathrm{A} \\
{\left[\mathrm{m}^{2}\right]}\end{array}$ & $\begin{array}{l}\text { Costo de capital } \\
\quad\left[\$ \mathrm{añono}^{-1}\right]\end{array}$ & $\begin{array}{c}\text { Costo de operación } \\
{\left[\$ \mathrm{año}^{-1}\right]}\end{array}$ \\
\hline 1 & $\mathrm{H} 1-\mathrm{C} 1$ & 17715.93 & 0.50 & 0.35 & 0.21 & 27.00 & 27.00 & 27.00 & 3186.99 & 225089.54 & - \\
\hline 3 & $\mathrm{H} 2 \mathrm{a}-\mathrm{C} 2$ & 1780.99 & 0.40 & 0.70 & 0.25 & 56.00 & 17.81 & 33.34 & 209.88 & 16691.68 & - \\
\hline 4 & $\mathrm{H} 2 \mathrm{~b}-\mathrm{C} 5 \mathrm{~b}$ & 7819.01 & 0.40 & 0.60 & 0.24 & 39.25 & 20.77 & 29.04 & 1122.06 & 80543.88 & - \\
\hline 5 & H3-C4 & 6600.00 & 0.14 & 0.14 & 0.07 & 44.30 & 44.30 & 44.30 & 2128.22 & 150975.68 & - \\
\hline 7 & $\mathrm{H} 4 \mathrm{~b}-\mathrm{C} 3$ & 18550.00 & 0.30 & 0.50 & 0.19 & 22.00 & 21.97 & 21.99 & 4499.67 & 316976.85 & - \\
\hline 8 & H1-C2 & 7249.01 & 0.50 & 0.70 & 0.29 & 11.28 & 42.35 & 23.49 & 1058.12 & 76068.36 & - \\
\hline HU5 & HU-C5 & 23839.14 & 0.50 & 0.60 & 0.27 & 30.00 & 69.20 & 46.90 & 1863.78 & 132464.85 & 1430348.64 \\
\hline CU1 & $\mathrm{H} 1-\mathrm{CU}$ & 3735.06 & 0.50 & 0.50 & 0.25 & 47.35 & 25.00 & 34.99 & 426.94 & 31885.86 & 22410.35 \\
\hline CU3 & $\mathrm{H} 3-\mathrm{CU}$ & 2658.15 & 0.14 & 0.50 & 0.11 & 74.30 & 45.00 & 58.43 & 415.92 & 31114.57 & 15948.91 \\
\hline \multirow[t]{4}{*}{ CU4 } & $\mathrm{H} 4-\mathrm{CU}$ & 25165.93 & 0.30 & 0.50 & 0.19 & 77.91 & 30.00 & 50.20 & 2673.50 & 189145.29 & 150995.60 \\
\hline & & & & & \multicolumn{7}{|c|}{16364.56} \\
\hline & & \multicolumn{3}{|c|}{$\begin{array}{l}\text { Total de servicios de } \\
\text { calentamiento }[\mathrm{kW}]\end{array}$} & 23839.14 & & \multicolumn{4}{|c|}{ Costo total anual de operación $[\$ a n ̃ o-1]$} & 1619703.50 \\
\hline & & \multicolumn{3}{|c|}{$\begin{array}{l}\text { Total de servicios de } \\
\text { enfriamiento }[\mathrm{kW}]\end{array}$} & 31559.14 & & \multicolumn{4}{|c|}{ Costo total anual de la red $\left[\$ \mathrm{año}^{-1}\right]$} & $2,919,687.66$ \\
\hline
\end{tabular}

Área total estimada $\left[\mathrm{m}^{2}\right]$

16364.56

Cálculo de temperaturas

intermedias calientes

th1,1 149.84

\begin{tabular}{cc} 
th1,8 & 77.35 \\
\hline th2,3 & 156.37
\end{tabular}

th2,4 $\quad 160.77$

th2,3-4 160.00

$\begin{array}{ll}\text { th } 3,2 & 214.30 \\ \text { th } 3,5 & 104.30\end{array}$

th4,6 114.48

th4,7 $\quad 106.97$

th4,6-7 $\quad 107.91$
Cálculo de temperaturas intermedias frías

\begin{tabular}{cc}
$\mathrm{tc} 1,6$ & 122.84 \\
\hline $\mathrm{tc} 2,8$ & 138.56 \\
\hline $\mathrm{tc} 5,2$ & 182.01 \\
$\mathrm{tc} 5,4$ & 180.75 \\
$\mathrm{tc} 5,2-4$ & 180.80
\end{tabular}

\section{Cálculo de fracciones d} flujo

$\begin{array}{llll}\text { fh2 } 3 & 0.174929723 \text { fh } 2,4 & 0.825070277\end{array}$

\begin{tabular}{llll} 
fh4,6 & 0.125435502 & fh 4,7 & 0.874564498 \\
\hline
\end{tabular}

$\begin{array}{llll}\text { fc5,2 } & 0.040683578 & \text { fc5,4 } & 0.959316422\end{array}$ 


\section{A.6. Revisión del Caso de Estudio 5.6}

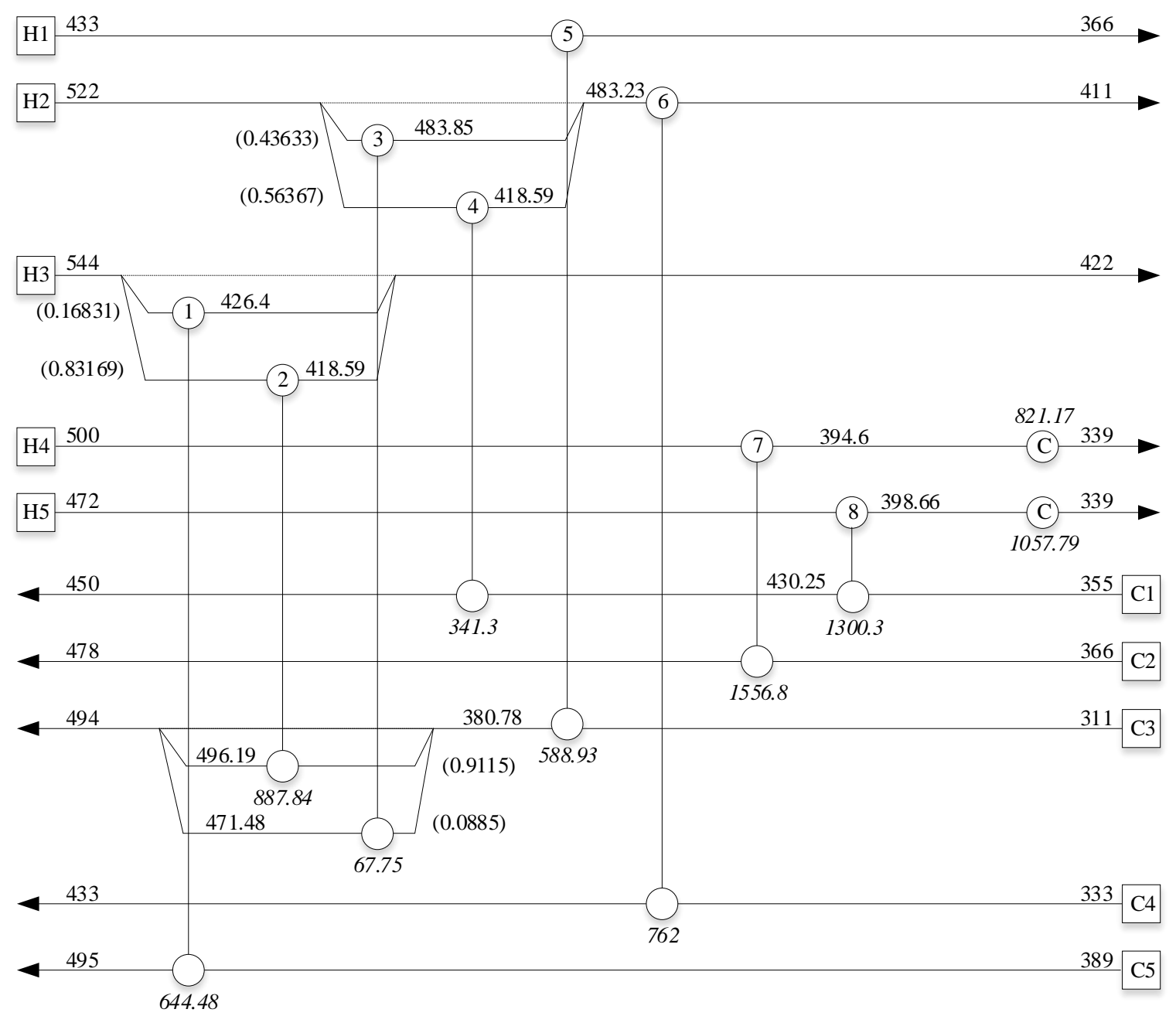

Figura A.14. Diseño de red determinado por Wu y col. (2015) para el Caso de Estudio 5.6 con $\mathrm{CTA}_{\mathrm{DMLT}}$ reportado de $\$ 42,961$ y $\mathrm{CTA}_{\mathrm{DMLT}}$ revisado de $\$ 43,319.28$. 
Tabla A.14 Revisión de costos del diseño de red de la Figura A.14 desarrollado por Wu y col. (2015) para el Caso de Estudio 5.6.

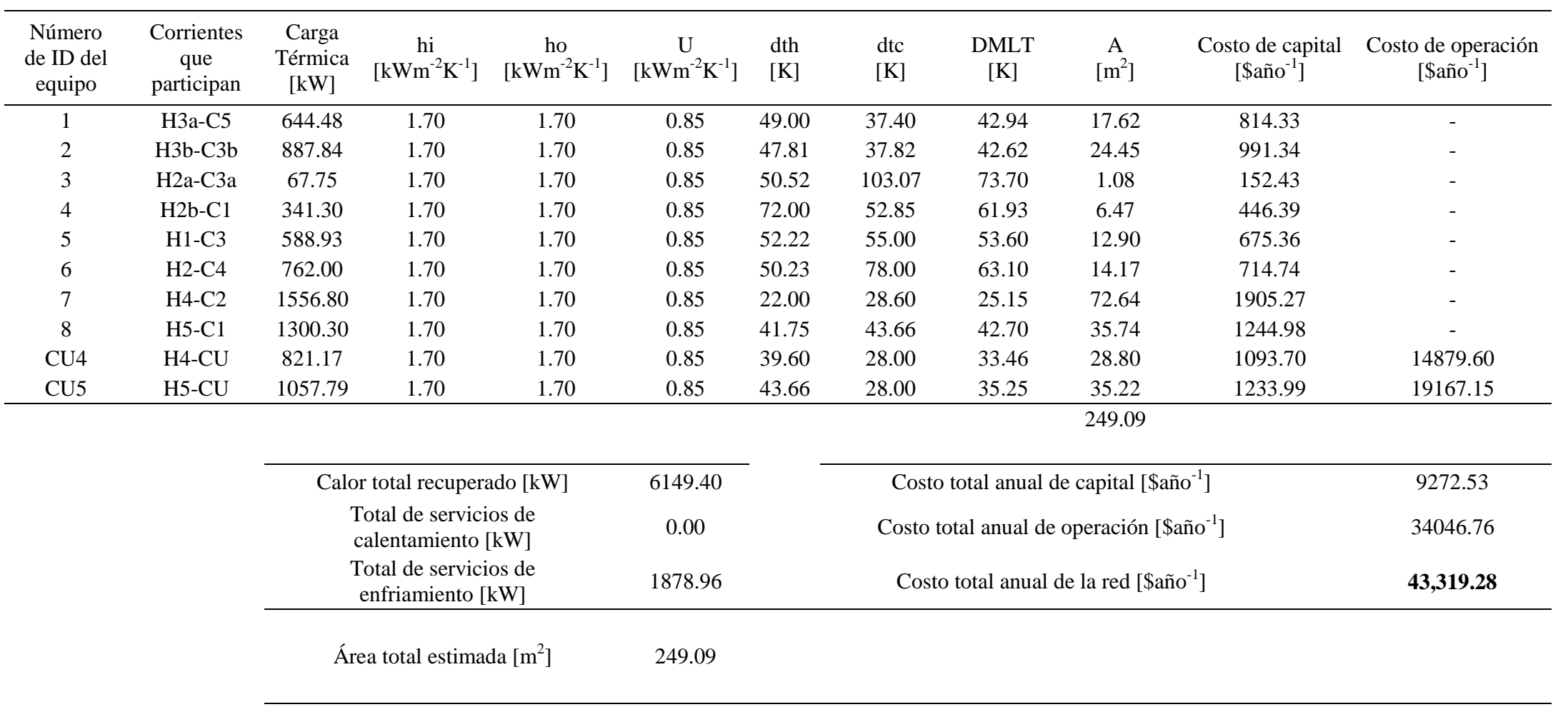

Cálculo de temperaturas intermedias calientes

\begin{tabular}{cc} 
th 2,3 & 426.40 \\
th 2,4 & 418.59 \\
th2,3-4 & 422.00 \\
\hline th3,1 & 483.85 \\
th 3,2 & 483.10 \\
th $3,1-2$ & 483.23 \\
\hline th 4,7 & 394.60 \\
\hline th5,8 & 398.66 \\
\hline
\end{tabular}

Cálculo de temperaturas intermedias frías

\begin{tabular}{cc}
$\mathrm{tc} 3,2$ & 496.19 \\
$\mathrm{tc} 3,3$ & 471.48 \\
$\mathrm{tc} 3,2-3$ & 494.00 \\
$\mathrm{tc} 3,5$ & 380.78 \\
\hline $\mathrm{tc} 1,8$ & 430.25 \\
\hline
\end{tabular}

Cálculo de fracciones de

flujo

\begin{tabular}{llll} 
fh2,3 & 0.436326265 & fh2,4 & 0.563673735 \\
fh3,1 & 0.168314401 & fh3,2 & 0.831685599 \\
\hline fc3,2 & 0.911498315 & fc3,3 & 0.088501685
\end{tabular}




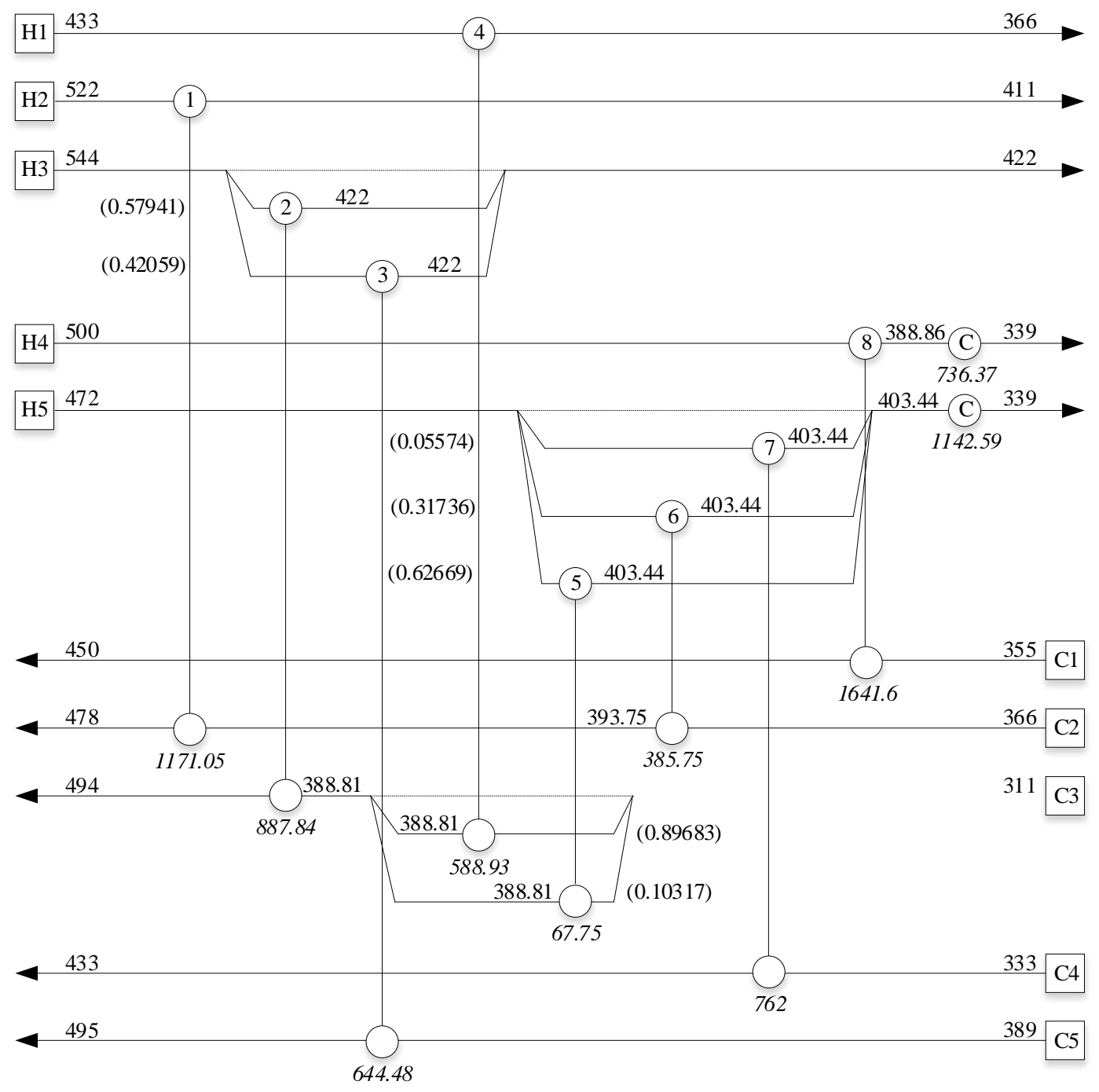

Figura A.15. Diseño de red determinado por Lin y Miller (2004) para el Caso de Estudio 5.6 con $\mathrm{CTA}_{\mathrm{DMLT}}$ reportado de $\$ 42,329$ y $\mathrm{CTA}_{\mathrm{DMLT}}$ revisado de $\$ 43,329.24$. 
Tabla A.15 Revisión de costos del diseño de red de la Figura A.14 desarrollado por Lin y Miller (2004) para el Caso de Estudio 5.6.

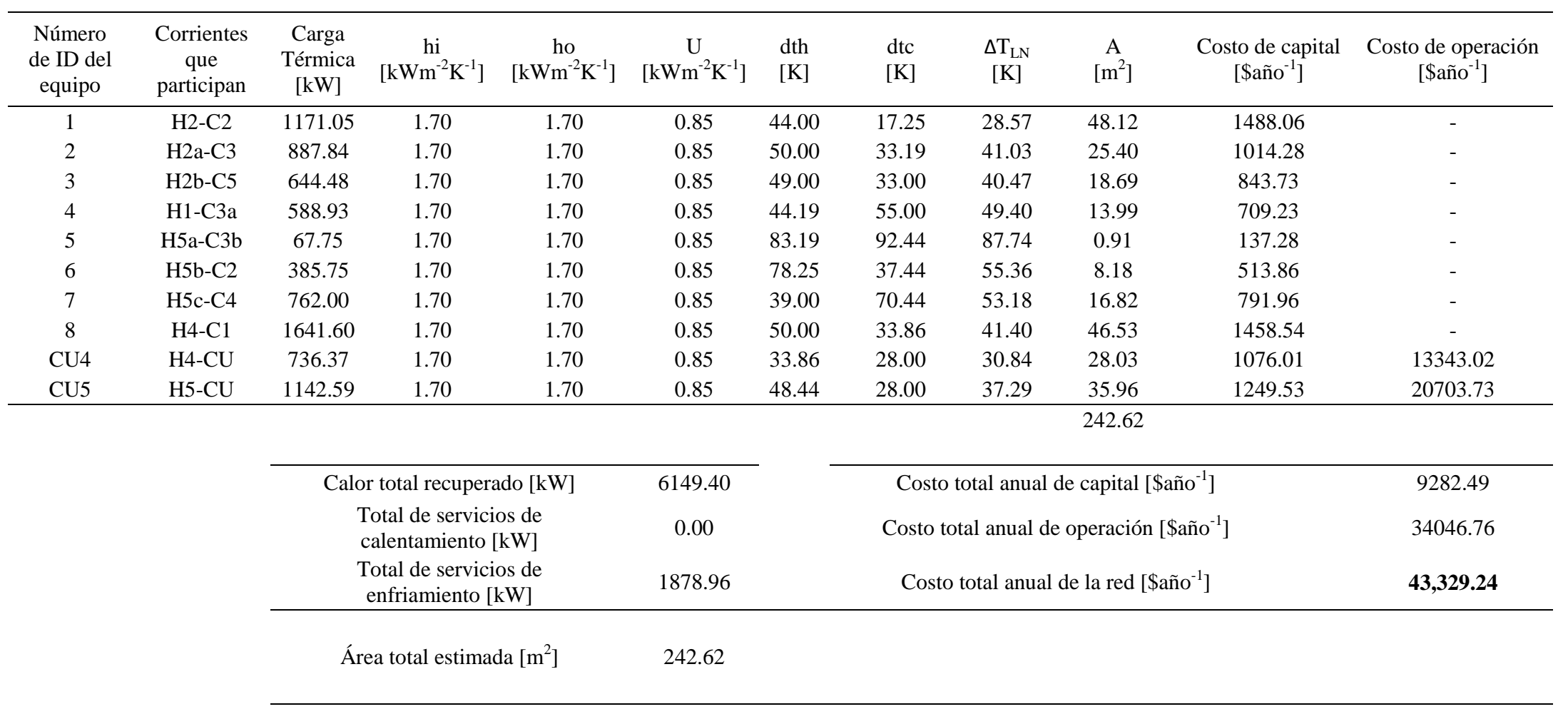

Cálculo de temperaturas intermedias calientes

\begin{tabular}{cc} 
th3,2 & 422.00 \\
th3,3 & 422.00 \\
th3,2-3 & 422.00 \\
\hline th 4,8 & 388.86 \\
\hline th5,5 & 403.44 \\
th5,6 & 403.44 \\
th5,7 & 403.44 \\
th5,6-7-8 & 403.44 \\
\hline
\end{tabular}

\section{Cálculo de temperaturas} intermedias frías

\begin{tabular}{cc}
$\mathrm{tc} 2,6$ & 393.75 \\
\hline $\mathrm{tc} 3,4$ & 388.81 \\
$\mathrm{tc} 3,5$ & 388.81 \\
$\mathrm{tc} 3,4-5$ & 388.81 \\
\hline
\end{tabular}

\section{Cálculo de fracciones de}

flujo

fh3,2 $0.579409001 \quad$ fh3,3 $\quad 0.420590999$

$\begin{array}{llllll}\text { fh5,5 } & 0.055738379 & \text { fh5,6 } & 0.317359111 & \text { fh5,7 } & 0.626902509\end{array}$




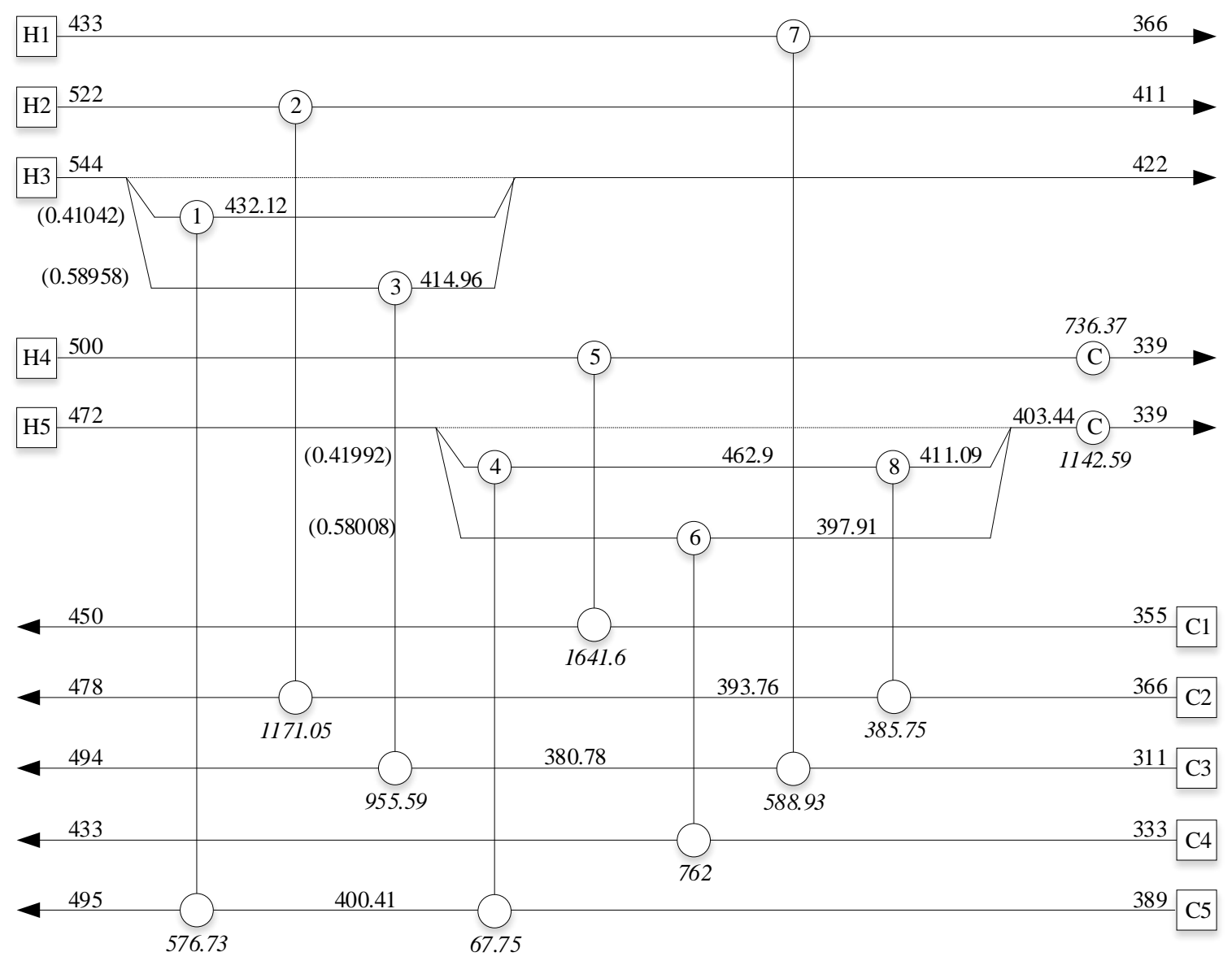

Figura A.16. Diseño de red determinado en este trabajo para el Caso de Estudio 5.6 con

CTA $_{\text {DMLT }}$ de $\$ 43,317.79$. 
Tabla A.16 Revisión de costos del diseño de red de la Figura A.15 desarrollado en este trabajo para el Caso de Estudio 5.6.

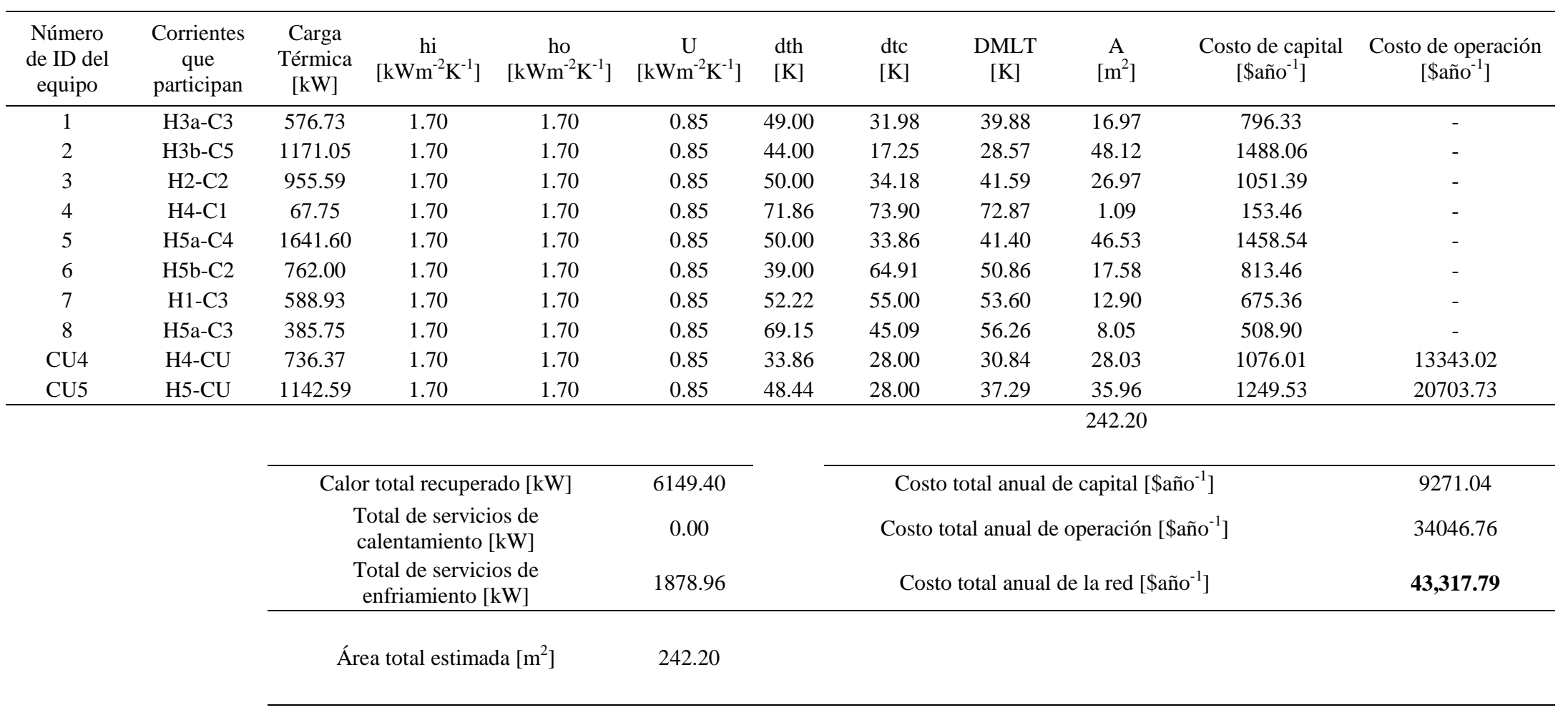

Cálculo de temperaturas intermedias calientes

\begin{tabular}{cc} 
th3,1 & 432.12 \\
th3,3 & 414.96 \\
\hline th4,5 & 388.86 \\
\hline th5,4 & 462.90 \\
th5,6 & 397.91 \\
th5,8 & 411.09 \\
th5,6-8 & 403.44388
\end{tabular}

Cálculo de temperaturas intermedias frías

\begin{tabular}{rr}
$\mathrm{tc} 2,8$ & 393.75 \\
\hline $\mathrm{tc} 3,7$ & 380.78 \\
\hline $\mathrm{tc} 5,4$ & 400.14
\end{tabular}

Cálculo de fracciones de

flujo

fh3,1 0.41041595 fh3,2 $\quad 0.58958405$




\section{A.7. Revisión del Caso de Estudio 5.7}

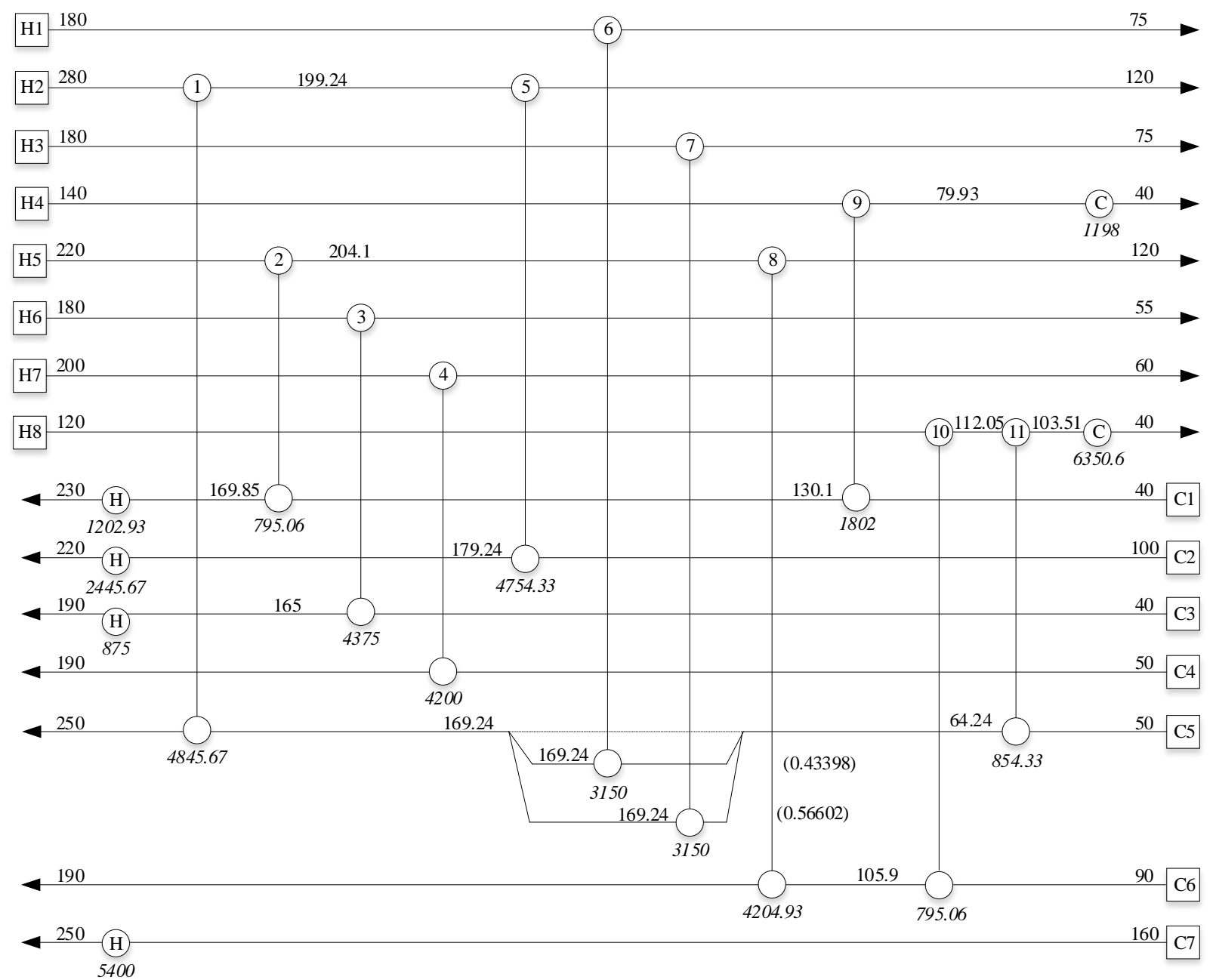

Figura A.17. Diseño de red determinado por Escobar y Trierweiler (2013) para el Caso de

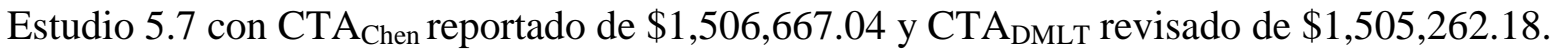


Tabla A.17 Revisión de costos del diseño de red de la Figura A.17 desarrollado por Escobar y Trierweiler (2013) para el Caso de Estudio 5.7.

\begin{tabular}{|c|c|c|c|c|c|c|c|c|c|c|c|}
\hline $\begin{array}{l}\text { Número } \\
\text { de ID del } \\
\text { equipo }\end{array}$ & $\begin{array}{l}\text { Corrientes } \\
\text { que } \\
\text { participan }\end{array}$ & $\begin{array}{c}\text { Carga } \\
\text { Térmica } \\
{[\mathrm{kW}]}\end{array}$ & $\begin{array}{c}\mathrm{hi} \\
{\left[\mathrm{kWm}^{-2 \circ} \mathrm{C}^{-1}\right]}\end{array}$ & $\begin{array}{c}\text { ho } \\
{\left[\mathrm{kWm}^{-2 \circ} \mathrm{C}^{-1}\right]}\end{array}$ & $\stackrel{\mathrm{U}}{\left[\mathrm{kWm}^{-2 \circ} \mathrm{C}^{-1}\right]}$ & $\begin{array}{l}\text { dth } \\
{\left[{ }^{\circ} \mathrm{C}\right]}\end{array}$ & $\begin{array}{c}\mathrm{dtc} \\
{\left[{ }^{\circ} \mathrm{C}\right]}\end{array}$ & $\begin{array}{c}\text { DMLT } \\
{\left[{ }^{\circ} \mathrm{C}\right]}\end{array}$ & $\begin{array}{c}\mathrm{A} \\
{\left[\mathrm{m}^{2}\right]}\end{array}$ & $\begin{array}{l}\text { Costo de capital } \\
\quad\left[\$ \text { año }^{-1}\right]\end{array}$ & $\begin{array}{c}\text { Costo de operación } \\
{\left[\$ \text { añno }^{-1}\right]}\end{array}$ \\
\hline 1 & $\mathrm{H} 2-\mathrm{C} 5$ & 4845.67 & 1.00 & 2.00 & 0.67 & 30.00 & 30.00 & 30.00 & 242.28 & 38705.27 & - \\
\hline 2 & $\mathrm{H} 5-\mathrm{C} 1$ & 795.07 & 1.00 & 1.00 & 0.50 & 50.15 & 74.00 & 61.30 & 25.94 & 13747.01 & - \\
\hline 3 & H6-C3 & 4375.00 & 2.00 & 2.00 & 1.00 & 15.00 & 15.00 & 15.00 & 291.67 & 43288.65 & - \\
\hline 4 & $\mathrm{H} 7-\mathrm{C} 4$ & 4200.00 & 0.40 & 2.00 & 0.33 & 10.00 & 10.00 & 10.00 & 1260.00 & 113742.10 & - \\
\hline 5 & $\mathrm{H} 2-\mathrm{C} 2$ & 4754.33 & 1.00 & 1.00 & 0.50 & 20.00 & 20.00 & 20.00 & 475.43 & 58908.11 & - \\
\hline 6 & H1-C5a & 3150.00 & 2.00 & 2.00 & 1.00 & 10.76 & 10.76 & 10.76 & 292.72 & 43384.12 & - \\
\hline 7 & $\mathrm{H} 3-\mathrm{C} 5 \mathrm{~b}$ & 3150.00 & 2.00 & 2.00 & 1.00 & 10.76 & 10.76 & 10.76 & 292.72 & 43384.12 & - \\
\hline 9 & $\mathrm{H} 4-\mathrm{C} 1$ & 1802.00 & 1.00 & 1.00 & 0.50 & 9.90 & 39.93 & 21.53 & 167.36 & 31265.43 & - \\
\hline 10 & H8-C6 & 795.07 & 0.50 & 1.00 & 0.33 & 14.10 & 22.05 & 17.78 & 134.16 & 27710.05 & - \\
\hline 11 & $\mathrm{H} 8-\mathrm{C} 5$ & 854.33 & 0.50 & 2.00 & 0.40 & 47.81 & 53.51 & 50.60 & 42.21 & 16279.42 & - \\
\hline HU1 & HU-C1 & 1202.94 & 1.00 & 1.00 & 0.50 & 95.00 & 155.15 & 122.62 & 19.62 & 12661.12 & 96234.80 \\
\hline HU2 & HU-C2 & 2445.67 & 1.00 & 1.00 & 0.50 & 105.00 & 145.76 & 124.27 & 39.36 & 15857.25 & 195653.60 \\
\hline HU3 & HU-C3 & 875.00 & 1.00 & 2.00 & 0.67 & 135.00 & 160.00 & 147.15 & 8.92 & 10580.67 & 70000.00 \\
\hline HU7 & HU-C7 & 5400.00 & 1.00 & 3.00 & 0.75 & 75.00 & 165.00 & 114.15 & 63.08 & 19191.06 & 432000.00 \\
\hline CU4 & $\mathrm{H} 4-\mathrm{CU}$ & 1198.00 & 1.00 & 2.00 & 0.67 & 39.93 & 15.00 & 25.46 & 70.57 & 20174.09 & 11980.00 \\
\hline CU8 & $\mathrm{H} 8-\mathrm{CU}$ & 6350.61 & 0.50 & 2.00 & 0.40 & 63.51 & 15.00 & 33.61 & 472.34 & 58659.20 & 63506.05 \\
\hline & & \multicolumn{3}{|c|}{ Calor total recuperado $[\mathrm{kW}]$} & 32926.40 & \multicolumn{5}{|c|}{ Costo total anual de capital $\left[\$\right.$ año $\left.^{-1}\right]$} & 635887.73 \\
\hline & & \multicolumn{3}{|c|}{$\begin{array}{l}\text { Total de servicios de } \\
\text { calentamiento }[\mathrm{kW}]\end{array}$} & 9923.61 & \multicolumn{5}{|c|}{ Costo total anual de operación $\left[\$\right.$ año $\left.^{-1}\right]$} & 869374.45 \\
\hline & & \multicolumn{3}{|c|}{$\begin{array}{l}\text { Total de servicios de } \\
\text { enfriamiento }[\mathrm{kW}]\end{array}$} & 7548.61 & \multicolumn{5}{|c|}{ 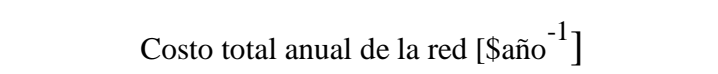 } & $1,505,262.18$ \\
\hline & & \multicolumn{3}{|c|}{ Área total estimada $\left[\mathrm{m}^{2}\right]$} & 4494.87 & & & & & & \\
\hline
\end{tabular}

Cálculo de temperaturas intermedias calientes

\begin{tabular}{cc} 
th2,1 & 199.24 \\
\hline th 4,9 & 79.93 \\
\hline th5,2 & 204.10 \\
\hline th 8,10 & 112.05
\end{tabular}

\begin{tabular}{cc}
$\begin{array}{c}\text { Cálculo de temperaturas } \\
\text { intermedias frías } \\
\text { tc } 1,2\end{array}$ & 169.85 \\
$\mathrm{tc} 1,9$ & 130.10 \\
\hline $\mathrm{tc} 2,5$ & 179.24 \\
\hline $\mathrm{tc} 3,3$ & 165.00 \\
\hline $\mathrm{tc} 5,6$ & 169.24 \\
$\mathrm{tc} 5,7$ & 169.24 \\
$\mathrm{tc} 5,6-7$ & 169.24 \\
$\mathrm{tc} 5,11$ & 64.24 \\
\hline $\mathrm{tc} 6,10$ & 105.90 \\
\hline
\end{tabular}

\section{Cálculo de fracciones de} flujo

$\begin{array}{llll}\text { tc } 1,7 & 0.50 & \text { tc } 1,8 & 0.50\end{array}$ 


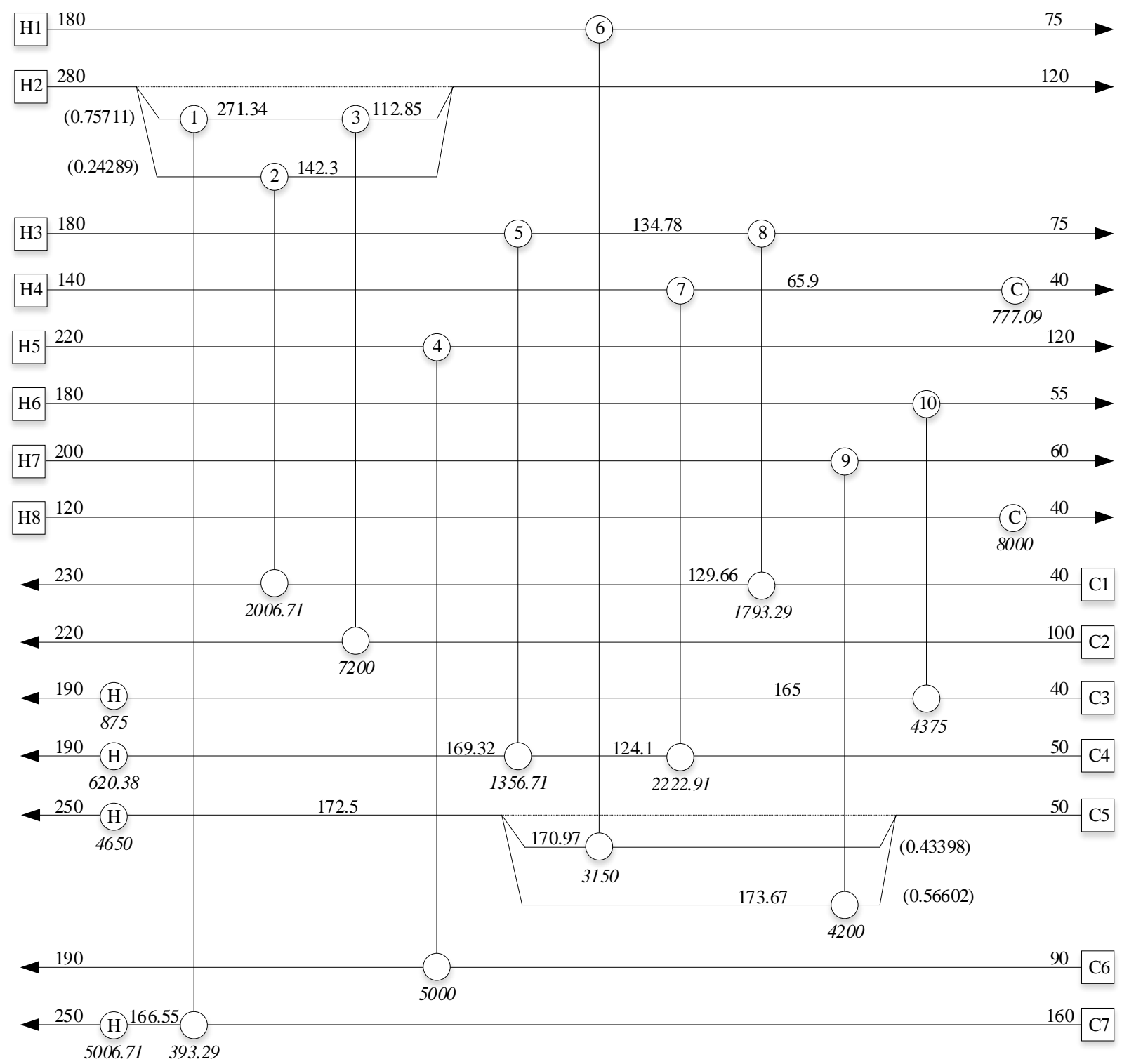

Figura A.18. Diseño de red determinado en este trabajo para el Caso de Estudio 5.7 con $\mathrm{CTA}_{\text {DMLT }}$ de $\$ 1,519,049.24$. 
Tabla A.18 Revisión de costos del diseño de red de la Figura A.18 desarrollado en este trabajo para el Caso de Estudio 5.7.

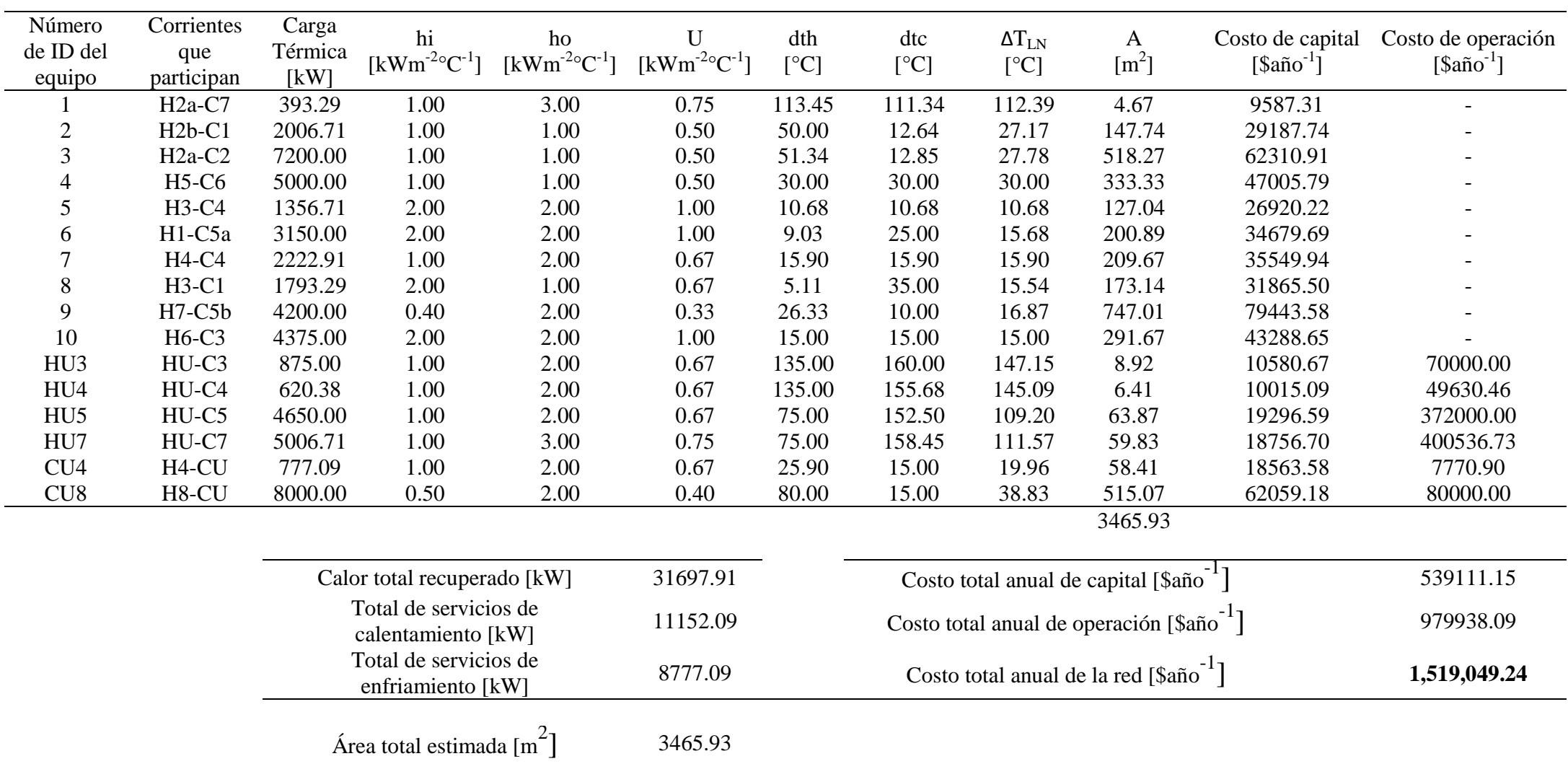

Cálculo de temperaturas

intermedias calientes

th2,1 271.34

th2,2 142.30

\begin{tabular}{ll} 
th2,3 & 112.85 \\
\hline th3,5 & 134.78
\end{tabular}

th4,7 65.90
Cálculo de temperaturas intermedias frías

\begin{tabular}{cc} 
tc 1,8 & 129.66 \\
\hline tc 3,10 & 165.00 \\
\hline tc 4,7 & 124.10 \\
tc 4,5 & 169.32 \\
\hline tc5,6 & 170.97 \\
tc5,9 & 173.67 \\
tc5,6-9 & 172.50 \\
\hline tc7,1 & 166.55
\end{tabular}

Cálculo de fracciones de

flujo

fh1,1 0.757111301 fh1,6 0.242888699

$\begin{array}{llll}\text { tc } 1,7 & 0.433981815 & \text { tc } 1,8 & 0.566018185\end{array}$ 


\section{Referencias}

Anantharaman, R.; Gundersen, T. (2006). Developments in the Sequential Framework for Heat Exchanger Network Synthesis of industrial size problems. Computer Aided Chemical Engineering, 21, 725-730.

Anantharaman, R.; Nastad, I.; Nygreen, B.; Gundersen, T. (2010). The sequential framework for heat exchanger network synthesis-The minimum number of units sub-problem. Computers and Chemical Engineering, 34(11), 1822-1830.

Bergamini, M. L.; Scenna, N. J.; Aguirre, P. A. (2007). Global optimal structures of heat exchanger networks by piecewise relaxation. Industrial and engineering chemistry research, $46(6), 1752-1763$.

Biegler, L. T.; Grossmann, I. E.; Westerberg, A. W. (1997). Systematic methods for chemical process design.

Björk, K. M.; Nordman, R. (2005). Solving large-scale retrofit heat exchanger network synthesis problems with mathematical optimization methods. Chemical Engineering and Processing: Process Intensification, 44(8), 869-876.

Björk, K. M.; Pettersson, F. (2003). Optimization of Large-scale Heat Exchanger Network Synthesis Problems. Modelling and Simulation, 2003, 313-318.

Björk, K. M.; Westerlund, T. (2002). Global optimization of heat exchanger network synthesis problems with and without the isothermal mixing assumption. Computers and chemical engineering, 26(11), 1581-1593.

Bochenek, R.; Jezowski, J.; Bartman, S. (2007). Optimization of heat exchanger network with fixed topology by genetic algorithms. Chem. Eng. Trans, 12, 195-200.

Briones, V.; Kokossis, A. C. (1999). Hypertargets: a Conceptual Programming approach for the optimisation of industrial heat exchanger networks-I. Grassroots design and network complexity. Chemical Engineering Science, 54(4), 519-539. 
Broeck, H. T. (1944). Economic selection of exchanger sizes. Industrial and Engineering Chemistry, 36(1), 64-67.

Cerda, J.; Westerberg, A. W. (1983). Synthesizing heat exchanger networks having restricted stream/stream matches using transportation problem formulations. Chemical Engineering Science, 38(10), 1723-1740.

Cerda, J.; Westerberg, A. W.; Mason, D.; Linnhoff, B. (1983). Minimum utility usage in heat exchanger network synthesis A transportation problem. Chemical Engineering Science, 38(3), 373-387.

Chakraborty, S.; Ghosh, P. (1999). Heat exchanger network synthesis: the possibility of randomization. Chemical Engineering Journal, 72(3), 209-216.

Chen, J. J. J. (1987). Comments on improvements on a replacement for the logarithmic mean. Chemical Engineering Science, 42(10), 2488-2489.

Chen, J.; Cui, G.; Duan, H. (2017). Multipopulation differential evolution algorithm based on the opposition-based learning for heat exchanger network synthesis. Numerical Heat Transfer, Part A: Applications, 72(2), 126-140.

Chen, X.; Li, Z.; Yang, J.; Shao, Z.; Zhu, L. (2008). Nested tabu search (TS) and sequential quadratic programming (SQP) method, combined with adaptive model reformulation for heat exchanger network synthesis (HENS). Industrial Engineering Chemistry Research, 47(7), 23202330.

Chen, Y.; Grossmann, I. E.; Miller, D. C. (2015). Computational strategies for large-scale MILP transshipment models for heat exchanger network synthesis. Computers \& Chemical Engineering, 82, 68-83.

Ciric, A. R.; Floudas, C. A. (1991). Heat exchanger network synthesis without decomposition. Computers and chemical engineering, 15(6), 385-396.

El-Halwagi, M. M. (1997). Pollution prevention through process integration: systematic design tools. Academic press.

El-Halwagi, M. M.; Manousiouthakis, V. (1989). Synthesis of mass exchange networks. AIChE Journal, 35(8), 1233-1244. 
Escobar, M.; Trierweiler, J. O. (2013). Optimal heat exchanger network synthesis: A case study comparison. Applied Thermal Engineering, 51(1), 801-826.

Floudas, C. A.; Ciric, A. R.; Grossmann, I. E. (1986). Automatic synthesis of optimum heat exchanger network configurations. AIChE Journal, 32(2), 276-290.

Flower, J. R.; Linnhoff, B. (1979). Thermodynamic analysis in the design of process networks. Computers and Chemical Engineering, 3(1), 283-291.

Furman, K. C.; Sahinidis, N. V. (2001). Computational complexity of heat exchanger network synthesis. Computers and Chemical Engineering, 25(9), 1371-1390.

Furman, K. C.; Sahinidis, N. V. (2002). A critical review and annotated bibliography for heat exchanger network synthesis in the 20th century. Industrial and Engineering Chemistry Research, 41(10), 2335-2370.

Gadalla, M. A. (2015). A new graphical method for Pinch Analysis applications: Heat exchanger network retrofit and energy integration. Energy, 81, 159-174.

Grossmann, I. E.; Sargent, R. W. H. (1978). Optimum design of heat exchanger networks. Computers Chemical Engineering, 2(1), 1-7.

Gundersen, T.; Naess, L. (1988). The synthesis of cost optimal heat exchanger networks: an industrial review of the state of the art. Computers and chemical engineering, 12(6), 503-530.

Gundersen, T.; Traedal, P.; Hashemi-Ahmady, A. (1997). Improved sequential strategy for the synthesis of near-optimal heat exchanger networks. Computers and chemical engineering, 21, S59-S64.

Gupta, A.; Ghosh, P. (2010). A randomized algorithm for the efficient synthesis of heat exchanger networks. Computers Chemical Engineering, 34(10), 1632-1639.

Hashemi-Ahmady, A.; Zamora, J. M.; Gundersen, T. (1999). A sequential framework for optimal synthesis of industrial size heat exchanger networks. PRES, 99, 329e334.

He, Q.; Cui, G. (2013). A principle of stream arrangement based on uniformity factor for heat exchanger networks synthesis. Applied Thermal Engineering, 61(2), 93-100.

Hohmann, E. C. (1971). Optimum networks for heat exchange (Doctoral dissertation, University of Southern California). 
Huang, K. F.; Karimi, I. A. (2013). Simultaneous synthesis approaches for cost-effective heat exchanger networks. Chemical Engineering Science, 98, 231-245.

Huang, K. F.; Al-mutairi, E. M.; Karimi, I. A. (2012). Heat exchanger network synthesis using a stagewise superstructure with non-isothermal mixing. Chemical engineering science, 73, 30-43.

Hwa, C. S. (1965). Mathematical formulation and optimization of heat exchanger networks using separable programming. In AIChE-IChemE Symposium Series (Vol. 4, pp. 101-106).

Jezowski, J. (1994a). Heat-Exchanger Network Grassroot And Retrofit Design.The Review Of the State-tf-the-Art: Part I. Heat-Exchanger Network Targeting and Insight Based Methods Of Synthesis. Hungarian Journal of Industrial Chemistry, 22(4), 279-294.

Jezowski, J. (1994b). Heat Exchanger Network Grassroot and Retrofit Design. The Review of the State-of-the-Art: Part II. Heat Exchanger Network Synthesis by Mathematical Methods and Approaches for Retrofit Design. Hungarian Journal of Industrial Chemistry, 22(4), 295-308.

Kesler, M. G.; Parker, R. O. (1969). Optimal networks of heat exchange. In Chem. Eng. Prog. Symp. Ser (Vol. 65, No. 92, pp. 111-120).

Klemeš, J. J. (Ed.). (2013). Handbook of process integration (PI): minimisation of energy and water use, waste and emissions. Elsevier.

Lewin, D. R. (1998). A generalized method for HEN synthesis using stochastic optimizationII.: The synthesis of cost-optimal networks. Computers chemical engineering, 22(10), 13871405.

Lewin, D. R.; Wang, H.; Shalev, O. (1998). A generalized method for HEN synthesis using stochastic optimization-I. general framework and MER optimal synthesis. Computers chemical engineering, 22(10), 1503-1513.

Li, G.; Luo, Y.; Xia, Y.; Hua, B. (2012). Improvement on the simultaneous optimization approach for heat exchanger network synthesis. Industrial and Engineering Chemistry Research, 51(18), 6455-6460.

Lin, B.; Miller, D. C. (2004). Solving heat exchanger network synthesis problems with Tabu Search. Computers chemical engineering, 28(8), 1451-1464. 
Linnhoff, B.; Ahmad, S. (1990). Cost optimum heat exchanger networks-1. Minimum energy and capital using simple models for capital cost. Computers Chemical Engineering, 14(7), 729750.

Linnhoff, B.; Flower, J. R. (1978a). Synthesis of heat exchanger networks: I. Systematic generation of energy optimal networks. AIChE Journal, 24(4), 633-642.

Linnhoff, B.; Flower, J. R. (1978b). Synthesis of heat exchanger networks: II. Evolutionary generation of networks with various criteria of optimality. AIChE Journal, 24(4), 642-654.

Linnhoff, B.; Hindmarsh, E. (1983). The pinch design method for heat exchanger networks. Chemical Engineering Science, 38(5), 745-763.

Luo, L. (2004). The identification and construction of the multistream heat exchanger networks. Computers and Applied Chemistry, 21(3), 333-338.

Luo, X.; Wen, Q. Y.; Fieg, G. (2009). A hybrid genetic algorithm for synthesis of heat exchanger networks. Computers Chemical Engineering, 33(6), 1169-1181.

Masso, A. H.; Rudd, D. F. (1969). The synthesis of system designs. II. Heuristic structuring. AIChE Journal, 15(1), 10-17.

Muraki, M.; Hayakawa, T. (1982). Practical synthesis method for heat exchanger network. Journal of Chemical Engineering of Japan, 15(2), 136-141.

Myankooh, Y. P.; Shafiei, S. (2015). Application of ACO R to find optimal no stream splitting heat exchanger networks for pre-designed heat exchanger networks. Chemical Engineering Research and Design, 96, 158-171.

Núñez-Serna, R. I.; Zamora, J. M. (2016). NLP model and stochastic multi-start optimization approach for heat exchanger networks. Applied Thermal Engineering, 94, 458-471.

Papoulias, S. A.; Grossmann, I. E. (1983). A structural optimization approach in process synthesis-II: Heat recovery networks. Computers and Chemical Engineering, 7(6), 707-721.

Pariyani, A.; Gupta, A.; Ghosh, P. (2006). Design of heat exchanger networks using randomized algorithm. Computers chemical engineering, 30(6), 1046-1053.

Paterson, W. R. (1984). A replacement for the logarithmic mean. Chemical Engineering Science, $39(11), 1635-1636$. 
Pavão, L. V.; Costa, C. B. B.; Ravagnani, M. A. S. S. (2017). Heat Exchanger Network Synthesis without stream splits using parallelized and simplified simulated Annealing and Particle Swarm Optimization. Chemical Engineering Science, 158, 96-107.

Pavão, L. V.; Costa, C. B. B.; Ravagnani, M. A. D. S. S.; Jiménez, L. (2017). Large-scale heat exchanger networks synthesis using simulated annealing and the novel rocket fireworks optimization. AIChE Journal, 63(5), 1582-1601.

Peng, F.; Cui, G. (2015). Efficient simultaneous synthesis for heat exchanger network with simulated annealing algorithm. Applied Thermal Engineering, 78, 136-149.

Petroleum, B. (2016). BP statistical review of world energy.

Pettersson, F. (2005). Synthesis of large-scale heat exchanger networks using a sequential match reduction approach. Computers and chemical engineering, 29(5), 993-1007.

Pettersson, F. (2008). Heat exchanger network design using geometric mean temperature difference. Computers and Chemical Engineering, 32(8), 1726-1734.

Pouransari, N.; Maréchal, F. (2015). Heat recovery networks synthesis of large-scale industrial sites: Heat load distribution problem with virtual process subsystems. Energy Conversion and Management, 89, 985-1000.

Quesada, I.; Grossmann, I. E. (1993). Global optimization algorithm for heat exchanger networks. Industrial and engineering chemistry research, 32(3), 487-499.

Rezaei, E.; Shafiei, S. (2008). An Efficient Coupled Genetic Algorithm-NLP Method for Heat Exchanger Network Synthesis. Iranian Journal of Chemical Engineering, 5(1), 22-33.

Ryoo, H. S.; Sahinidis, N. V. (1995). Global optimization of nonconvex NLPs and MINLPs with applications in process design. Computers and Chemical Engineering, 19(5), 551-566.

Shethna, H. K.; Jezowski, J. M.; Castillo, F. J. L. (2000). A new methodology for simultaneous optimization of capital and operating cost targets in heat exchanger network design. Applied thermal engineering, 20(15), 1577-1587.

Smith, R. (2005). Chemical process: design and integration. John Wiley and Sons.

Tan, R. R.; Foo, D. C. (2007). Pinch analysis approach to carbon-constrained energy sector planning. Energy, 32(8), 1422-1429. 
Tan, Y. L.; Ng, D. K.; El-Halwagi, M. M.; Foo, D. C.; Samyudia, Y. (2014). Floating pinch method for utility targeting in heat exchanger network (HEN). Chemical Engineering Research and Design, 92(1), 119-126.

Toffolo, A. (2009). The synthesis of cost optimal heat exchanger networks with unconstrained topology. Applied Thermal Engineering, 29(17), 3518-3528.

Towler, G. P.; Mann, R.; Serriere, A. J.; Gabaude, C. M. (1996). Refinery hydrogen management: cost analysis of chemically-integrated facilities. Industrial and engineering chemistry research, 35(7), 2378-2388.

Trivedi, K. K. (1988). The pinch design method for the synthesis of heat exchanger networks: the constrained case. In AIChE Annual Meeting, Washington, DC.

Umeda, T. A.; Itoh, J.; Shiroko, K. (1978). Heat-exchange system synthesis. Chemical Engineering Progress, 74(7), 70-76.

Underwood, A. (1970). Simple formula to calculate mean temperature difference. Chemical Engineering, 77(13), 192.

Verheyen, W.; Zhang, N. (2006). Design of flexible heat exchanger network for multi-period operation. Chemical engineering science, 61(23), 7730-7753.

Wang, J.; Cui, G.; Xiao, Y.; Luo, X.; Kabelac, S. (2017). Bi-level heat exchanger network synthesis with evolution method for structure optimization and memetic particle swarm optimization for parameter optimization. Engineering Optimization, 49(3), 401-416.

Wang, Y. P.; Smith, R. (1994). Wastewater minimisation. Chemical Engineering Science, 49(7), 981-1006.

Westbrook, G. T. (1961). Use this method to size each stage for best operation. Hydrocarbon Process and Petroleum Refinement, 40, 201-206.

Westerberg, A. W.; Shah, J. V. (1978). Assuring a global optimum by the use of an upper bound on the lower (dual) bound. Computers and Chemical Engineering, 2(2-3), 83-92.

Wu, H.; Yan, F.; Li, W.; Zhang, J. (2015). Simultaneous Heat Exchanger Network Synthesis Involving Nonisothermal Mixing Streams with Temperature-Dependent Heat Capacity. Industrial Engineering Chemistry Research, 54(36), 8979-8987. 
Yee, T. F.; Grossmann, I. E. (1990). Simultaneous optimization models for heat integration-II. Heat exchanger network synthesis. Computers and Chemical Engineering, 14(10), 1165-1184.

Yerramsetty, K. M.; Murty, C. V. S. (2008). Synthesis of cost-optimal heat exchanger networks using differential evolution. Computers Chemical Engineering, 32(8), 1861-1876.

Zamora, J. M.; Grossmann, I. E. (1997). A comprehensive global optimization approach for the synthesis of heat exchanger networks with no stream splits. Computers and chemical engineering, 21, S65-S70.

Zamora, J. M.; Grossmann, I. E. (1998a). A global MINLP optimization algorithm for the synthesis of heat exchanger networks with no stream splits. Computers and Chemical Engineering, 22(3), 367-384.

Zamora, J. M.; Grossmann, I. E. (1998b). Continuous global optimization of structured process systems models. Computers and chemical engineering, 22(12), 1749-1770.

Zargarzadeh, M.; Karimi, I. A.; Alfadala, H. (2007). Olexan: a tool for online exergy analysis. In 17th European symposium on computer-aided process engineering Bucharest, Romania, computer-aided chemical engineering (Vol. 24).

Zhang, H.; Cui, G.; Xiao, Y.; Chen, J. (2017). A novel simultaneous optimization model with efficient stream arrangement for heat exchanger network synthesis. Applied Thermal Engineering, 110, 1659-1673.

Zhaoyi, H.; Liang, Z.; Hongchao, Y.; Jianxiong, Y. (2013). Simultaneous synthesis of structuralconstrained heat exchanger networks with and without stream splits. The Canadian Journal of Chemical Engineering, 91(5), 830-842.

Zhelev, T. K.; Ntlhakana, J. L. (1999). Energy-environment closed-loop through oxygen pinch. Computers and Chemical Engineering, 23, S79-S83.

Zhu, X. X. (1997). Automated design method for heat exchanger network using block decomposition and heuristic rules. Computers and Chemical Engineering, 21(10), 1095-1104. 
SÍNTESIS DE REDES DE INTERCAMBIO DE CALOR MEDIANTE LA GENERACIÓN Y OPTIMIZACIÓN DE TOPOLOGÍAS REDUNDANTES

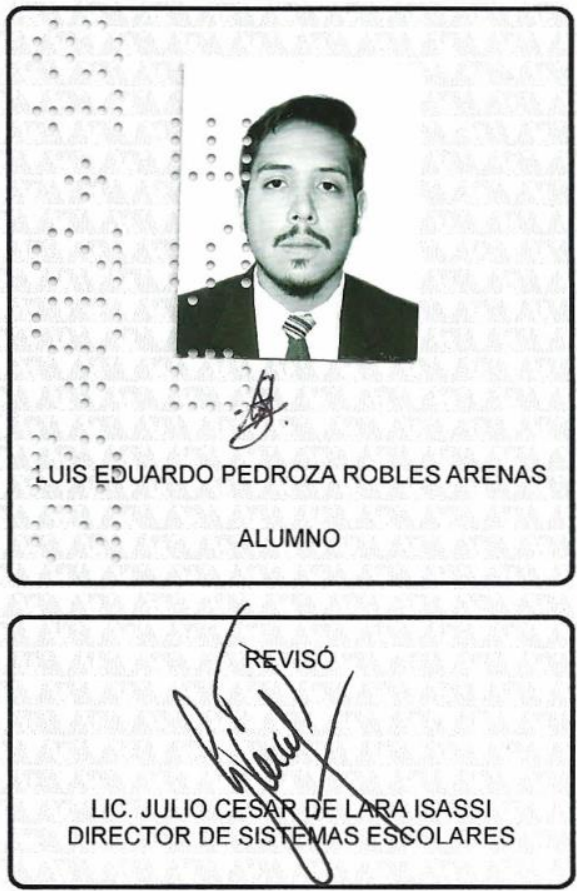

DIRECTOR DE LA DIVISIÓN DE CBI

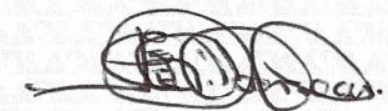

DR. JOSE GILBERTO CORDOBA HERRERA

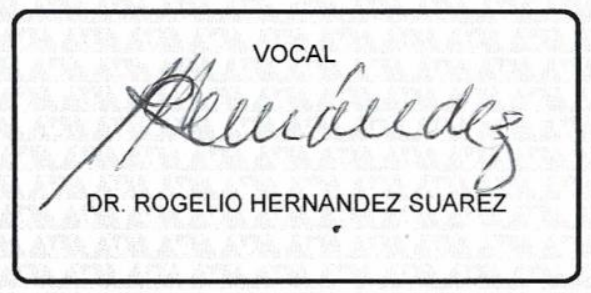

En la Ciudad de México, se presentaron a las 12:00 horas del día 20 del mes de febrero del año 2018 en la Unidad Iztapalapa de la Universidad Autónoma Metropolitana, los suscritos miembros del jurado:

DR. JUAN MANUEL ZAMORA MATA

DR. ROGELIO HERNANDEZ SUAREZ

DR. MIGUEL ANGEL GUTIERREZ LIMON

Bajo la Presidencia del primero y con carácter de Secretario el último, se reunieron para proceder al Examen de Grado cuya denominación aparece al margen, para la obtención del grado de:

MAESTRO EN CIENCIAS (INGENIERIA QUIMICA)

DE: LUIS EDUARDO PEDROZA ROBLES ARENAS

$y$ de acuerdo con el artículo 78 fracción III del Reglamento de Estudios Superiores de la Universidad Autónoma Metropolitana, los miembros del jurado resolvieron:

\section{APROBAR}

Acto continuo, el presidente del jurado comunicó al interesado el resultado de la evaluación $y$, en caso aprobatorio, le fue tomada la protesta. 